ANALYSIS \& PDE

Volume $4 \quad$ No. $1 \quad 2011$

JEAN-MARC BOUCLET

STRICHARTZ ESTIMATES ON ASYMPTOTICALLY HYPERBOLIC MANIFOLDS 


\title{
STRICHARTZ ESTIMATES ON ASYMPTOTICALLY HYPERBOLIC MANIFOLDS
}

\author{
JEAN-MARC BOUCLET
}

We prove local in time Strichartz estimates without loss for the restriction of the solution of the Schrödinger equation, outside a large compact set, on a class of asymptotically hyperbolic manifolds.

1. The results 1

2. The strategy of the proof of Theorem $1.2 \quad 7$

3. Estimates on the geodesic flow near infinity 22

4. The Hamilton-Jacobi and transport equations 32

5. An Isozaki-Kiada type parametrix 50

6. Geometric optics and Egorov's theorem on AH manifolds $\quad 55$

$\begin{array}{ll}\text { 7. Dispersion estimates } & 67\end{array}$

$\begin{array}{ll}\text { Appendix A. Control on the range of some diffeomorphisms } & 79\end{array}$

$\begin{array}{ll}\text { Acknowledgments } & 83\end{array}$

$\begin{array}{ll}\text { References } & 83\end{array}$

\section{The results}

Let $(\mathcal{M}, G)$ be a Riemannian manifold of dimension $n \geq 2$ with Riemannian volume density $d G$ and associated Laplace-Beltrami operator $\Delta_{G}$. The Strichartz estimates for the Schrödinger equation

$$
i \partial_{t} u+\Delta_{G} u=0, \quad u_{\mid t=0}=u_{0},
$$

are basically estimates of

$$
\|u\|_{L^{p}\left([0,1], L^{q}(\mathcal{M}, d G)\right)}:=\left(\int_{0}^{1}\|u(t, \cdot)\|_{L^{q}(\mathcal{M}, d G)}^{p} d t\right)^{1 / p},
$$

in terms of certain $L^{2}$ quantities of $u_{0}$, when the pair of exponents $(p, q)$ satisfies the admissibility conditions

$$
\frac{2}{p}+\frac{n}{q}=\frac{n}{2}, \quad p \geq 2, \quad(p, q) \neq(2, \infty) .
$$

Strichartz estimates play an important role in the proof of local existence results for nonlinear Schrödinger equations (see for instance [Ginibre and Velo 1985; Cazenave 2003; Burq et al. 2004]). We won't consider such applications in this paper and will only focus on the estimates themselves.

We review some classical results. If $\mathcal{M}=\mathbb{R}^{n}$ with the flat metric, it is well known [Strichartz 1977; Ginibre and Velo 1985; Keel and Tao 1998] that

$$
\|u\|_{L^{p}\left([0,1], L^{q}\left(\mathbb{R}^{n}\right)\right)} \lesssim\left\|u_{0}\right\|_{L^{2}\left(\mathbb{R}^{n}\right)} .
$$

MSC2000: 35B45, 35S30, 58J40, 58J47.

Keywords: Strichartz estimates, asymptotically hyperbolic, Isozaki-Kiada parametrix, semiclassical functional calculus. 
In this model case, the time interval $[0,1]$ can be replaced by $\mathbb{R}$ and the Strichartz estimates are said to be global in time. Furthermore, the conditions (1-2) are seen to be natural by considering the action of the scaling $u(t, x) \mapsto u\left(t / \lambda^{2}, x / \lambda\right)$ on both Schrödinger equation and Strichartz estimates.

(In this paper we will not pursue global in time Strichartz estimates. Although one can expect that they exist, it is not clear how to obtain them by the present method. One may hope to obtain such global in time results at least for initial data spectrally cutoff on the low frequencies by combining the present analysis with the method of [Bouclet and Tzvetkov 2008].)

In more general situations, estimates of the form (1-3) sometimes have to be replaced by

$$
\|u\|_{L^{p}\left([0,1], L^{q}(M, d G)\right)} \lesssim\left\|u_{0}\right\|_{H^{s}(\mu, d G)}, \quad s \geq 0
$$

where

$$
\left\|u_{0}\right\|_{H^{s}(\mathcal{M}, d G)}:=\left\|\left(1-\Delta_{G}\right)^{s / 2} u_{0}\right\|_{L^{2}(\mathcal{M}, d G)},
$$

is the natural $L^{2}$ Sobolev norm. If $s>0$, estimates such as (1-4) are called Strichartz estimates with loss (of $s$ derivatives). Notice that, under fairly general assumptions on $(\mathcal{M}, G)$, we have the Sobolev embeddings $H^{s}(\mathcal{M}, d G) \subset L^{q}(\mathcal{M}, d G)$ for $s>n / 2-n / q$. They show that (1-4) holds automatically if $s$ is large enough and the point of Strichartz estimates with loss (and a fortiori without loss) is to consider smaller $s$ than those given by Sobolev embeddings.

Such inequalities have been proved by Bourgain [1993] for the flat tori $\mathbb{T}^{1}$ and $\mathbb{T}^{2}$, for certain values of $p, q$ and any $s>0$ (i.e., with "almost no loss"), and by Burq, Gérard, and Tzvetkov [Burq et al. 2004] for any compact manifold with $s=1 / p$. The techniques of the latter work are actually very robust and can be applied to prove the same results on many noncompact manifolds; the estimates are known to be sharp for $M=\mathbb{S}^{3}$ with $p=2$ and by considering certain subsequences of eigenfunctions of the Laplacian. This counterexample can then be used to construct quasimodes and show that (1-4) cannot hold in general with $s=0$, even for noncompact manifolds.

A natural question is therefore to find (sufficient) conditions leading to estimates with no loss.

A classical one is the nontrapping condition. We recall that $(\mathcal{M}, G)$ is nontrapping if all geodesics escape to infinity (implying that $\mathcal{M}$ is noncompact). It was for instance shown in [Staffilani and Tataru 2002; Robbiano and Zuily 2005; Bouclet and Tzvetkov 2007] that, for nontrapping perturbations of the flat metric on $\mathbb{R}^{n},(1-4)$ holds with $s=0$. By a perturbation we mean that the departure of $G$ from the flat metric $G_{\text {Eucl }}$ is small near infinity and we refer to those papers for more details. In [Hassell et al. 2006], the more general case of nontrapping asymptotically conic manifolds was considered. To emphasize the difference with the asymptotically hyperbolic manifolds studied in this paper, we simply recall that $(\mathcal{M}, G)$ is asymptotically conic if $G$ is close to $d r^{2}+r^{2} g$, in a neighborhood of infinity diffeomorphic to $(R,+\infty) \times S$, for some fixed metric $g$ on a compact manifold $S$. The asymptotically Euclidean case corresponds to the case where $S=\mathbb{S}^{n-1}$.

The nontrapping condition, however, has several drawbacks, such as being nongeneric and difficult to check. Moreover, it is not clearly a necessary condition to get Strichartz estimates without loss.

In [Bouclet and Tzvetkov 2007], we partially got rid of this condition by considering Strichartz estimates localized near spatial infinity. For long-range perturbations $G$ of the Euclidean metric on $M=\mathbb{R}^{n}$ (meaning that $\partial_{x}^{\alpha}\left(G(x)-G_{\text {Eucl }}\right)=\mathscr{O}\left(\langle x\rangle^{-\tau-|\alpha|}\right)$ for $\tau>0$ ), trapping or not, we proved the existence of 
$R>0$ large enough such that, if $\chi \in C_{0}^{\infty}\left(\mathbb{R}^{n}\right)$ satisfies $\chi \equiv 1$ for $|x| \leq R$, then

$$
\|(1-\chi) u\|_{L^{p}\left([0,1] ; L^{q}\left(\mathbb{R}^{n}, d G\right)\right)} \lesssim\left\|u_{0}\right\|_{L^{2}\left(\mathbb{R}^{n}, d G\right)} .
$$

This shows that the possible loss in Strichartz estimates can only come from a bounded region where the metric is essentially arbitrary (recall that being asymptotically Euclidean is only a condition at infinity). One can loosely interpret this result as follows: as long as the metric is close to a model one for which one has Strichartz estimates without loss, the solution to the Schrödinger equation satisfies Strichartz estimates without loss too.

The first goal of the present paper is to show that the same result holds in (bounded) negative curvature, more precisely for asymptotically hyperbolic (AH) manifolds. We point out, however, that even if our Theorem 1.2 below is formally the same as in the asymptotically Euclidean case [Bouclet and Tzvetkov 2007, Theorem 1], its proof involves new arguments using the negative curvature. One of the messages of this paper is that, by taking advantage of certain curvature effects described at the end of this Section, we prove Strichartz estimates using long time (microlocal) parametrices of the Schrödinger group which are localized in very narrow regions of the phase space, much smaller than those considered in the asymptotically Euclidean situation.

As far as the Schrödinger equation is concerned, Strichartz estimates on negatively curved spaces have been studied in [Banica 2007; Pierfelice 2006; 2008; Anker and Pierfelice 2009] (see [Tataru 2001] for the wave equation). In [Pierfelice 2006], Pierfelice considers perturbations of the Schrödinger equation on the hyperbolic space $\mathbb{H}^{n}$ by singular time-dependent radial potentials, with radial initial data (and also radial source terms) and derives some weighted Strichartz estimates without loss. The nonradial case for the free Schrödinger equation on $\mathbb{\boxplus}^{n}$ is studied in [Banica 2007] where weighted Strichartz estimates are obtained too. The more general case of certain Lie groups, namely Damek-Ricci spaces, was considered in [Pierfelice 2008] for global in time estimates (see also [Banica et al. 2008] for the two-dimensional case) and further generalized in [Banica and Duyckaerts 2007]. In these last papers, only radial data are considered. This radial assumption was removed in [Anker and Pierfelice 2009]. This last paper also shows, with [Banica et al. 2008], in such geometries, the set of admissible pairs for the Strichartz estimates is contained in a triangle, and thus is much wider than in the (asymptotically) Euclidean case. One expects that such a result remains valid in our context, but this does not clearly follow from the tools presented here and might require refined propagation estimates.

In this article, we give a proof of Strichartz estimates at infinity which is purely (micro)local and so, to a large extent, stable under perturbation. We do not use any Lie group structure or spherical symmetry, nor do we assume any nontrapping condition. We refer to Definition 1.1 below for precise statements and simply quote here that our class of manifolds contains $\mathbb{t}^{n}$, some of its quotients and perturbations thereof. In particular, we do not assume that the curvature is constant, even near infinity. (Powerful microlocal techniques for AH manifolds have already been developed by Melrose and his school; see [Mazzeo and Melrose 1987] and the references in [Melrose 1995]. These geometric methods, based on compactification and blowup considerations, are perfectly designed for conformally compact manifolds with boundary, but do not clearly apply to the more general manifolds we study here.)

In the next few pages we fix our framework and state our main results precisely, highlighting the key points that allow us to prove them. We conclude the section with an overview of the remainder of the article, on page 7 . 
Definition 1.1 (AH manifold). ( $\left.\mathcal{M}^{n}, G\right)$ is asymptotically hyperbolic if there exist a compact set $\mathscr{K} \Subset \mathcal{M}$, a real number $R_{\mathscr{K}}>0$, a compact manifold without boundary $S$ and a function

$$
r \in C^{\infty}(\mathcal{M}, \mathbb{R}) \quad \text { with } \quad r(m) \rightarrow+\infty \text { as } m \rightarrow \infty
$$

(a coordinate near $\overline{\mathcal{M} \backslash \mathscr{K}}$ ) such that we have an isometry

$$
\Psi:(M \backslash \mathscr{K}, G) \rightarrow\left(\left(R_{\mathscr{K}},+\infty\right)_{r} \times S, d r^{2}+e^{2 r} g(r)\right),
$$

where $g(r)$ is a family of metrics on $S$ depending smoothly on $r$ such that, for some $\tau>0$ and some fixed metric $g$ on $S$, we have

$$
\left\|\partial_{r}^{k}(g(r)-g)\right\|_{C^{\infty}\left(S, T^{*} S \otimes T^{*} S\right)} \lesssim r^{-\tau-k} \quad \text { for } r>R_{\mathscr{K}},
$$

for all $k \geq 0$ and all seminorms $\|\cdot\|_{C^{\infty}\left(S, T^{*} S \otimes T^{*} S\right)}$ in the space of smooth sections of $T^{*} S \otimes T^{*} S$.

With no loss of generality, we can assume that the decay rate $\tau$ in (1-8) satisfies

$$
0<\tau<1
$$

Therefore, by analogy with the standard terminology in Euclidean scattering, $d r^{2}+e^{2 r} g(r)$ can be considered as a long-range perturbation of the metric $d r^{2}+e^{2 r} g$. Notice that the conformally compact case quoted above corresponds to the special situation where $g(r)$ is of the form $\tilde{g}\left(e^{-r}\right)$, for some family of metrics $(\tilde{g}(x))_{0 \leq x \ll 1}$ depending smoothly on $x \in\left[0, x_{0}\right)\left(x_{0}\right.$ small enough) up to $x=0$. In that case, $g(r)$ is an exponentially small perturbation of $g=\tilde{g}(0)$. The assumption (1-8) is therefore more general.

We next denote by $\Delta_{G}$ the Laplace-Beltrami operator associated to this metric. It is classical that this operator is essentially self-adjoint on $C_{0}^{\infty}(M)$ (using for instance the method of [Helffer and Robert 1983]), and therefore generates a unitary group $e^{i t \Delta_{G}}$ on $L^{2}(M, d G)$.

Our main result is the following.

Theorem 1.2. There exists $\chi \in C_{0}^{\infty}(\mathcal{M})$, with $\chi \equiv 1$ on a sufficiently large compact set, such that, for all pair $(p, q)$ satisfying (1-2),

$$
\left\|(1-\chi) e^{i t \Delta_{G}} u_{0}\right\|_{L^{p}\left([0,1] ; L^{q}(\mathcal{M}, d G)\right)} \lesssim\left\|u_{0}\right\|_{L^{2}(\mathcal{M}, d G)}, \quad u_{0} \in C_{0}^{\infty}(\mathcal{M}) .
$$

This theorem is the AH analogue of Theorem 1 of [Bouclet and Tzvetkov 2007] in the asymptotically Euclidean case.

To be more complete, let us point out that the analysis contained in this paper and a classical argument due to [Staffilani and Tataru 2002] (see also [Bouclet and Tzvetkov 2007, Section 5]), using the local smoothing effect [Doi 1996], would give the following global in space estimates.

Theorem 1.3. If in addition $(M, G)$ is nontrapping, then we have global in space Strichartz estimates with no loss: for all pair $(p, q)$ satisfying (1-2),

$$
\left\|e^{i t \Delta_{G}} u_{0}\right\|_{L^{p}\left([0,1] ; L^{q}(\mathcal{M}, d G)\right)} \lesssim\left\|u_{0}\right\|_{L^{2}(\mathcal{M}, d G)}, \quad u_{0} \in C_{0}^{\infty}(\mathcal{M}) .
$$

We state this result as a theorem although we won't explicitly prove it. The techniques are fairly well known and don't involve any new argument in the present context. We simply note that resolvent estimates implying the local smoothing effect can be found in [Cardoso and Vodev 2002]. 
Remark. Theorem 1.2 reduces the proof of potential improvements of Burq-Gérard-Tzvetkov inequalities to local in space estimates of the form

$$
\|\chi u\|_{L^{p}\left([0,1], L^{q}(\mathcal{M}, d G)\right)} \lesssim\left\|u_{0}\right\|_{H^{s}(\mathcal{M}, d G)},
$$

with $0 \leq s<1 / p$. It would be interesting to know if such inequalities holds for some trapping $\mathrm{AH}$ manifolds.

We now describe, quite informally, the key points of the analysis developed in this paper. Assuming for simplicity that $S=\mathbb{S}^{1}$ (and thus $n=2$ ), we consider the model case where the principal symbol of the Laplacian is

$$
p=\rho^{2}+e^{-2 r} \eta^{2} .
$$

For convenience, we introduce

$$
P:=-e^{(n-1) r / 2} \Delta_{G} e^{-(n-1) r / 2}=-e^{r / 2} \Delta_{G} e^{-r / 2},
$$

which is self-adjoint with respect to $d r d \theta$, instead of $e^{(n-1) r} d r d \theta=e^{r} d r d \theta$ for the Laplacian itself.

Recall first that, by the Keel-Tao $T T^{*}$ Theorem [1998], proving Strichartz estimates (without loss) mainly reduces to prove certain dispersion estimates. Using the natural semiclassical time scaling $t \mapsto h t$, this basically requires to control the propagator $e^{-i t h P}$ for semiclassical times of order $h^{-1}$. Such a control on the full propagator is out of reach (basically because of trapped trajectories) but, fortunately, studying some of its cutoffs will be sufficient.

After fairly classical reductions, we will work with semiclassical pseudodifferential operators localized where $r \gg 1$ and $p \in I, I$ being a (relatively) compact interval of $(0,+\infty)$. We can split the latter region into two areas defined by

$$
\Gamma^{+}=\left\{r \gg 1, p \in I, \rho>-\frac{1}{2} p^{1 / 2}\right\}, \quad \Gamma^{-}=\left\{r \gg 1, p \in I, \rho<\frac{1}{2} p^{1 / 2}\right\},
$$

respectively called the outgoing and incoming areas. The main interest of such areas is that one has a very good control on the geodesic flow therein (see Section 3). Basically, geodesics with initial data in outgoing (resp. incoming) areas escape to infinity as $t \rightarrow+\infty$ (resp. $t \rightarrow-\infty$ ), which is proved in Proposition 3.3. One thus expects to be able to give long time approximations of the propagator $e^{-i t h P}$, microlocalized in such areas, for large times ( $t \geq 0$ in outgoing areas and $t \leq 0$ in incoming ones).

In the asymptotically Euclidean case, it turns out that one can give accurate approximations of $e^{-i t h P} \chi^{ \pm}$ for times $t$ such that $0 \leq \pm t \lesssim h^{-1}$, if $\chi^{ \pm}$are pseudodifferential cutoffs localized in $\Gamma^{ \pm}$. This is not the AH case: here we are only able to approximate $e^{-i t h P} \chi_{\mathrm{s}}^{ \pm}$for cutoffs $\chi_{\mathrm{s}}^{ \pm}$localized in much smaller areas, namely

$$
\Gamma_{\mathrm{s}}^{+}(\varepsilon)=\left\{r \gg 1, p \in I, \rho>\left(1-\varepsilon^{2}\right) p^{1 / 2}\right\}, \quad \Gamma_{\mathrm{s}}^{-}(\varepsilon)=\left\{r \gg 1, p \in I, \rho<\left(\varepsilon^{2}-1\right) p^{1 / 2}\right\},
$$

which we call strongly outgoing/incoming areas. Here $\varepsilon$ will be a fixed small real number. We then obtain approximations of the form

$$
e^{-i t h P} \chi_{\mathrm{s}}^{ \pm}=J_{S^{ \pm}}\left(a^{ \pm}\right) e^{-i t h D_{r}^{2}} J_{S^{ \pm}}\left(b^{ \pm}\right)^{*}+\mathcal{O}\left(h^{N}\right), \quad 0 \leq \pm t \lesssim h^{-1} .
$$


Here $e^{-i t h D_{r}^{2}}$ is the semiclassical group associated to the radial part $D_{r}^{2}$ of $P$. Here and in the sequel, we shall use the standard notation

$$
D_{r}=i^{-1} \partial_{r}, \quad D_{\theta}=i^{-1} \partial_{\theta}
$$

The operators $J_{S^{ \pm}}\left(a^{ \pm}\right)$and $J_{S^{ \pm}}\left(b^{ \pm}\right)$are Fourier integral operators with amplitudes $a^{ \pm}, b^{ \pm}$supported in strongly outgoing $(+) /$ incoming $(-)$ areas and phases essentially of the form

$$
S^{ \pm} \approx r \rho+\theta \eta+\frac{e^{-2 r} \eta^{2}}{4 \rho}
$$

i.e., the sum of the free phase $r \rho+\theta \eta$ and of a term whose Hessian is nondegenerate in $\eta$, which will be crucial for the final stationary phase argument (the small factor $e^{-2 r}$ will be eliminated by a change of variable). The nondegeneracy of the full phase of the parametrix (1-11) in $\rho$ will come of course from $e^{-i t h D_{r}^{2}}$. This approximation of $S^{ \pm}$comes basically from (4-34) and (4-35). Although the right-hand side does not depend on \pm , it is only defined in the disconnected regions $\{\rho>0\}$ and $\{\rho<0\}$.

The approximation (1-11) is the AH Isozaki-Kiada parametrix and a significant part of this paper is devoted to its construction. We mention that it is an adaptation to the AH geometry of an approximation introduced first in [Isozaki and Kitada 1985] to study perturbations of the Euclidean Laplacian by longrange potentials. In the present paper, it will be used very similarly to the usual (semiclassical) Euclidean one as in [Bouclet and Tzvetkov 2007]. Its main interest is to give microlocal approximations of the propagator for times of size $h^{-1}$. Recall however the big difference with the asymptotically Euclidean case where one is able to consider cutoffs supported in $\Gamma^{ \pm}$rather than $\Gamma_{\mathrm{s}}^{ \pm}(\varepsilon)$ in the AH case. We therefore have to consider the left parts, namely

$$
\Gamma_{\text {inter }}^{ \pm}=\Gamma^{ \pm} \backslash \Gamma_{\mathrm{s}}^{ \pm}(\varepsilon)
$$

which we call intermediate areas. These areas will only contribute to the dispersion estimates for small times, in view of the following argument. By choosing $\delta$ small enough and by splitting the interval $\left(-\frac{1}{2}, 1-\varepsilon^{2}\right)$ into small intervals of size $\delta$, we can write

$$
\Gamma_{\text {inter }}^{ \pm}=\bigcup_{l \lesssim \delta^{-1}}\left\{r \gg 1, p \in I, \pm \rho p^{-1 / 2} \in\left(\sigma_{l}, \sigma_{l}+\delta\right)\right\}=\bigcup_{l \lesssim \delta^{-1}} \Gamma_{\text {inter }}^{ \pm}(l, \varepsilon, \delta) .
$$

Carefuly consideration of the Hamiltonian flow $\Phi_{p}^{t}$ of $p$ shows that, for any fixed (small) time $t_{0}$, we can choose $\delta$ (which depends also on $\varepsilon$ ) such that

$$
\Phi_{p}^{t}\left(\Gamma_{\text {inter }}^{ \pm}(l, \varepsilon, \delta)\right) \cap \Gamma_{\text {inter }}^{ \pm}(l, \varepsilon, \delta)=\varnothing \quad \text { for } \pm t \geq t_{0}
$$

By semiclassical propagation, this implies that

$$
\chi_{\text {inter }}^{ \pm} e^{-i t h P} \chi_{\text {inter }}^{ \pm *}=\mathcal{O}\left(h^{\infty}\right) \text { for } \pm t \geq t_{0},
$$

for pseudodifferential operators $\chi_{\text {inter }}^{ \pm}$localized in $\Gamma_{\text {inter }}^{ \pm}(l, \varepsilon, \delta)$. Such operators typically appear in the $T T^{*}$ argument and the estimate above reduces the proof of dispersion estimates to times $|t| \leq t_{0}$. The latter range of times can then be treated by fairly standard geometric optics approximation.

We interpret (1-12) as a negative curvature effect on the geodesic flow, which we can roughly describe as follows, say in the outgoing case. For initial conditions $(r, \theta, \rho, \eta)$ in $\Gamma_{\text {inter }}^{+}(l, \varepsilon, \delta)$, the bounds 
$\frac{1}{2}<\rho \leq\left(1-\varepsilon^{2}\right) p^{1 / 2}$ yield the lower bound

$$
\dot{\rho}^{t}=2 e^{-2 r^{t}}\left(\eta^{t}\right)^{2} \gtrsim \varepsilon^{2},
$$

over a sufficiently long time, if we set $\left(r^{t}, \theta^{t}, \rho^{t}, \eta^{t}\right)=: \Phi_{p}^{t}$. This ensures that $\rho^{t} / p^{1 / 2}$ increases fast enough to leave the interval $\left(\sigma_{l}, \sigma_{l}+\delta\right)$ before $t=t_{0}$ and give (1-12). In the asymptotically flat case, that is, with $r^{-2}$ instead of $e^{-2 r}$, we have $\dot{\rho}^{t}=2\left(r^{t}\right)^{-3}\left(\eta^{t}\right)^{2}$ and its control from below is not as good, basically because of the "extra" third power of $\left(r^{t}\right)^{-1}$.

Overview of remaining sections. In Section 2, we introduce all the necessary definitions, and some additional results, needed to prove Theorem 1.2. The latter proof is given in Section $2 \mathrm{E}$ using microlocal approximations which will be proved in Sections 5, 6 and 7 .

In Section 3, we study the properties of the geodesic flow in outgoing/incoming areas required to construct the phases involved in the Isozaki-Kiada parametrix. This parametrix is then constructed in Section 5.

In Section 6 we prove two results: the small semiclassical time approximation of the Schrödinger group by the WKB method and the propagation of the microlocal support (Egorov theorem). These results are essentially well known. We need however to check that all the symbols and phases belong to the natural classes (for AH geometry) of Definition 2.2 below. Furthermore, we use our Egorov theorem to obtain a propagation property in a time scale of size $h^{-1}$, which is not quite standard.

Finally, in Section 7, we prove dispersion estimates using basically stationary phase estimates in the parametrices obtained in Sections 5 and 6.

Up to the semiclassical functional calculus, which is taken from [Bouclet 2007; Bouclet 2010] and whose results are recalled in Section 2C, this paper is essentially self-contained. This is not only for the reader's convenience, but also because the results of Section 6 do require proofs in the AH setting, although they are in principle well known. The construction of Section 5 is new.

\section{The strategy of the proof of Theorem 1.2}

2A. The setup. Before discussing the proof of Theorem 1.2, we give the form of the Laplacian, volume densities and related objects on AH manifolds.

The isometry (1-7) defines polar coordinates: $r$ is the radial coordinate and $S$ will be called the angular manifold. Coordinates on $S$ will be denoted by $\theta_{1}, \ldots, \theta_{n-1}$.

A finite atlas on $\mathcal{M} \backslash \mathscr{K}$ is obtained as follows. By (1-7), we have a natural projection $\pi_{S}:(\mathcal{M} \backslash \mathscr{K}, G) \rightarrow S$ defined as the second component of $\Psi$, that is,

$$
\Psi(m)=\left(r(m), \pi_{S}(m)\right) \in\left(R_{\mathscr{K}},+\infty\right) \times S \text { for } m \in \mathcal{M} \backslash \mathscr{K} .
$$

Choosing a finite cover of the angular manifold by coordinate patches $U_{\iota}$, say

$$
S=\bigcup_{l \in \mathscr{I}} U_{l}
$$

with corresponding diffeomorphisms

$$
\psi_{\iota}: U_{\iota} \rightarrow \psi_{\iota}\left(U_{\iota}\right) \subset \mathbb{R}^{n-1},
$$


we consider the open sets

$$
u_{\iota}:=\Psi^{-1}\left(\left(R_{\mathscr{L}},+\infty\right) \times U_{\iota}\right) \subset \mathcal{M} \backslash \mathscr{K}
$$

and then define diffeomorphisms

$$
\Psi_{\iota}: U_{\iota} \rightarrow\left(R_{\mathscr{K}},+\infty\right) \times \psi_{\iota}\left(U_{\iota}\right) \subset \mathbb{R}^{n},
$$

by

$$
\Psi_{\iota}(m)=\left(r(m), \psi_{\iota}\left(\pi_{S}(m)\right)\right) .
$$

The collection $\left(U_{\iota}, \Psi_{\iota}\right)_{l \in \mathscr{I}}$ is then an atlas on $\mathcal{M} \backslash \mathscr{K}$. If $\theta_{1}, \ldots, \theta_{n-1}$ are the coordinates in $U_{\iota}$, that is, $\psi_{\iota}=\left(\theta_{1}, \ldots, \theta_{n-1}\right)$, the coordinates in $u_{\iota}$ are then $\left(r, \theta_{1}, \ldots, \theta_{n-1}\right)$.

We now give formulas for the Riemannian measure $d G$ and the Laplacian $\Delta_{G}$ on $M \backslash \mathscr{K}$. In local coordinates $\theta=\left(\theta_{1}, \ldots, \theta_{n-1}\right)$ on $S$, the Riemannian density associated to $g(r)$ reads

$$
d g(r):=\operatorname{det}(g(r, \theta))^{1 / 2}\left|d \theta_{1} \wedge \cdots \wedge d \theta_{n-1}\right|,
$$

where $\operatorname{det}(g(r, \theta))=\operatorname{det}\left(g_{j k}(r, \theta)\right)$ if $g(r)=g_{j k}(r, \theta) d \theta_{j} d \theta_{k}$ (using the summation convention). Then, in local coordinates on $M \backslash \mathscr{K}$, the Riemannian density is

$$
d G=e^{(n-1) r} \operatorname{det}(g(r, \theta))^{1 / 2}\left|d r \wedge d \theta_{1} \wedge \cdots \wedge d \theta_{n-1}\right| .
$$

Now consider the Laplacian. Slightly abusing the notation, we set

$$
c(r, s)=\frac{1}{2} \frac{\partial_{r} \operatorname{det}(g(r, s))}{\operatorname{det}(g(r, s))} \quad \text { for } r>R_{\mathscr{K}}, s \in S,
$$

since, for fixed $r$, the quotient of $\partial_{r} \operatorname{det}\left(g_{j k}(r, \theta)\right)$ by $2 \operatorname{det}\left(g_{j k}(r, \theta)\right)$ is intrinsically defined as a function on $S$, independently of the choice of the coordinate chart. We then have

$$
\Delta_{G}=\partial_{r}^{2}+e^{-2 r} \Delta_{g(r)}+c(r, s) \partial_{r}+(n-1) \partial_{r} .
$$

It will turn out be convenient to work with the density

$$
\widehat{d G}=e^{(1-n) r} d G,
$$

rather than $d G$ itself. In particular, we will use the following elementary property: for all relatively compact subset $V_{\iota}^{\prime} \Subset \psi_{\iota}\left(U_{\iota}\right)$, all $R>R_{\mathscr{K}}$ and all $1 \leq q \leq \infty$, we have the equivalence of norms

$$
\|u\|_{L^{q}(\mathcal{M}, \widehat{d G})} \approx\left\|u \circ \Psi_{\iota}^{-1}\right\|_{L^{q}\left(\mathbb{R}^{n}\right)}, \quad \operatorname{supp}(u) \subset \Psi_{\iota}^{-1}\left((R,+\infty) \times V_{\iota}^{\prime}\right),
$$

$L^{q}\left(\mathbb{R}^{n}\right)$ being the usual Lebesgue space. This is a simple consequence of (1-8) and (2-5) (we consider $R>R_{\mathscr{K}}$ since (1-8) gives an upper bound for $\operatorname{det} g(r, \theta)$ as $r \rightarrow R_{\mathscr{K}}$, not a lower bound).

We then have a unitary isomorphism

$$
L^{2}(\mathcal{M}, \widehat{d G}) \ni u \mapsto e^{-(n-1) r / 2} u \in L^{2}(\mathcal{M}, d G),
$$

and $\Delta_{G}$ is unitarily equivalent to the operator

$$
\widehat{\Delta}_{G}:=e^{\gamma_{n} r} \Delta_{G} e^{-\gamma_{n} r}, \quad \gamma_{n}=\frac{n-1}{2},
$$


on $L^{2}(\mathcal{M}, \widehat{d G})$. This operator reads

$$
\widehat{\Delta}_{G}=\partial_{r}^{2}+e^{-2 r} \Delta_{g(r)}+c(r, s) \partial_{r}-\gamma_{n} c(r, s)-\gamma_{n}^{2},
$$

and we will work with

$$
P=-\widehat{\Delta}_{G}-\gamma_{n}^{2} .
$$

If $q_{\iota}(r, ., \cdot)$ is the principal symbol of $-\Delta_{g(r)}$ in the chart $U_{\iota}$, namely

$$
q_{\iota}(r, \theta, \xi)=\sum_{1 \leq k, l \leq n-1} g^{k l}(r, \theta) \xi_{k} \xi_{l}
$$

the principal symbol of $P$ in the chart $U_{\iota}$ is then

$$
p_{\iota}=\rho^{2}+e^{-2 r} q_{\iota}(r, \theta, \eta),=\rho^{2}+q_{\iota}\left(r, \theta, e^{-r} \eta\right) .
$$

The full symbol of $P$ is of the form $p_{\iota}+p_{\iota, 1}+p_{\iota, 0}$ with

$$
p_{\iota, j}=\sum_{k+|\beta|=j} a_{l, k \beta}(r, \theta) \rho^{k}\left(e^{-r} \eta\right)^{\beta}, \quad j=0,1 .
$$

The terms of degree 1 in $\eta$ come from the first-order terms of the symbol of $-\Delta_{g(r)}$. In the expression of $\Delta_{G}$ they carry a factor $e^{-2 r}$ and therefore, if $j=1, k=0$ and $|\beta|=1$ above, we could write $a_{l, k \beta}(r, \theta)=e^{-r} b_{l, k \beta}(r, \theta)$ for some function $b_{l, k \beta}$ bounded as $r \rightarrow \infty$. This remark and (1-8) show more precisely that, for all $V \Subset \psi_{\iota}\left(U_{\iota}\right)$, the coefficients in (2-15) decay as

$$
\left|\partial_{r}^{j} \partial_{\theta}^{\alpha} a_{l, k \beta}(r, \theta)\right| \leq C_{V j \alpha}\langle r\rangle^{-\tau-1-j}, \quad \theta \in V, r \geq R_{\mathscr{K}}+1 .
$$

The decay rate $-\tau-1-j$ will be important to solve transport equations for the Isozaki-Kiada parametrix. This is the main reason of the long-range assumption (1-8).

2B. Pseudodifferential operators and the spaces $\mathscr{P}_{\mathrm{hyp}}(\Omega)$. We will consider $h$-pseudodifferential operators ( $h$ - $\Psi$ DOs) in a neighborhood of infinity and the calculus will be rather elementary. For instance, we will only consider compositions of operators with symbols supported in the same coordinate patch and no invariance result under diffeomorphism will be necessary.

The first step is to construct a suitable partition of unity near infinity. Using the cover (2-2) and the related diffeomorphisms (2-3), we consider a partition of unity on $S$ of the form

and a function $\kappa \in C^{\infty}(\mathbb{R})$ such that

$$
\sum_{\iota \in \Phi} \kappa_{\iota} \circ \psi_{\iota}=1, \quad \text { with } \kappa_{\iota} \in C_{0}^{\infty}\left(\mathbb{R}^{n-1}\right), \operatorname{supp}\left(\kappa_{\iota}\right) \Subset \psi_{\iota}\left(U_{\iota}\right),
$$

$$
\operatorname{supp}(\kappa) \subset\left[R_{\mathscr{K}}+1,+\infty\right), \quad \kappa \equiv 1 \quad \text { on }\left[R_{\mathscr{K}}+2,+\infty\right) .
$$

Then, the functions $\left(\kappa \otimes \kappa_{\iota}\right) \circ \Psi_{\iota} \in C^{\infty}(\mathcal{M})$ satisfy

$$
\sum_{\iota \in \mathscr{I}}\left(\kappa \otimes \kappa_{\iota}\right) \circ \Psi_{\iota}(m)= \begin{cases}1 & \text { if } r(m) \geq R_{\mathscr{\digamma}}+2, \\ 0 & \text { if } r(m) \leq R_{\mathscr{\digamma}}+1,\end{cases}
$$


which means that they define a partition of unity near infinity. We could obtain a partition of unity on $\mu$ by adding a finite number of compactly supported functions (in coordinate patches) be we won't need it since the whole analysis in this paper will be localized near infinity.

We also consider $\tilde{\kappa} \in C^{\infty}(\mathbb{R})$ and $\tilde{\kappa}_{\iota} \in C_{0}^{\infty}\left(\mathbb{R}^{n-1}\right)$, for all $\iota \in \mathscr{I}$, such that

$$
\begin{aligned}
& \tilde{\kappa} \equiv 1 \quad \text { on }\left(R_{\mathscr{K}}+\frac{1}{2},+\infty\right), \quad \operatorname{supp}(\tilde{\kappa}) \subset\left(R_{\mathscr{K}}+\frac{1}{4},+\infty\right), \\
& \tilde{\kappa}_{l} \equiv 1 \quad \text { near } \operatorname{supp}\left(\kappa_{l}\right), \quad \operatorname{supp}\left(\tilde{\kappa}_{l}\right) \Subset \psi_{l}\left(U_{l}\right) \text {. }
\end{aligned}
$$

We next choose, for each $\iota \in \mathscr{I}$, two relatively compact open subsets $V_{\iota}$ and $V_{\iota}^{\prime}$ such that

$$
\operatorname{supp}\left(\kappa_{\iota}\right) \Subset V_{\iota} \Subset V_{\iota}^{\prime} \Subset \operatorname{supp}\left(\tilde{\kappa}_{l}\right) \quad \text { and } \quad \tilde{\kappa}_{\iota} \equiv 1 \text { near } V_{l}^{\prime} .
$$

We are now ready to define our $\Psi$ DOs. In the following definition, we will say that $a \in C^{\infty}\left(\mathbb{R}^{2 n}\right)$ is a symbol if either $a \in C_{b}^{\infty}\left(\mathbb{R}^{2 n}\right)$ — that is, $a$ is bounded with all derivatives bounded —or

$$
a(r, \theta, \rho, \eta)=\sum a_{k \beta}(r, \theta) \rho^{k} \eta^{\beta},
$$

with $a_{k \beta} \in C_{b}^{\infty}\left(\mathbb{R}^{n}\right)$, the sum being finite. We shall give examples below. Notice that throughout this paper, $\rho$ and $\eta$ will denote respectively the dual variables to $r$ and $\theta$.

Definition 2.1. For $\iota \in \mathscr{I}$, all $h \in(0,1]$ and all symbol $a$ such that

$$
\operatorname{supp}(a) \subset\left[R_{\mathscr{C}}+1,+\infty\right) \times V_{\iota}^{\prime} \times \mathbb{R}^{n},
$$

we define

$$
\widehat{O p}_{\iota}(a): C_{0}^{\infty}(\mathcal{M}) \rightarrow C^{\infty}(\mathcal{M})
$$

by

$$
\left(\widehat{O p}_{\iota}(a) u\right) \circ \Psi_{\iota}^{-1}(r, \theta)=a\left(r, \theta, h D_{r}, h D_{\theta}\right)\left(\tilde{\kappa}(r) \tilde{\kappa}_{\iota}(\theta)\left(u \circ \Psi_{\iota}^{-1}\right)(r, \theta)\right) .
$$

Note the cutoff $\tilde{\kappa} \otimes \tilde{\kappa}_{\iota}$ in the right-hand side of (2-24). It makes the Schwartz kernel of $\widehat{O p}_{\iota}(a)$ supported in a closed subset of $\mathcal{M}^{2}$ strictly contained in the patch $U_{\iota}^{2}$ so that $\widehat{O p}_{\iota}(a)$ is fully defined by the prescription of $\Psi_{\iota *} \widehat{O p}_{\iota}(a) \Psi_{\iota}^{*}$. For future reference, we recall that the kernel of the latter operator is

$$
(2 \pi h)^{-n} \iint e^{\frac{i}{h}\left(r-r^{\prime}\right) \rho+\frac{i}{h}\left(\theta-\theta^{\prime}\right) \cdot \eta} a(r, \theta, \rho, \eta) d \rho d \eta \tilde{\chi}\left(r^{\prime}\right) \tilde{\chi}_{\iota}\left(\theta^{\prime}\right) .
$$

The notation $\widehat{O p}$, refers to the following relation with the measure $\widehat{d G}$ : if $a \in C_{b}^{\infty}\left(\mathbb{R}^{2 n}\right)$ satisfies (2-23), then

$$
\left\|\widehat{O P}_{\iota}(a)\right\|_{L^{2}(\mathcal{M}, \widehat{d G}) \rightarrow L^{2}(\mathcal{M}, \widehat{d G})} \lesssim 1, \quad h \in(0,1] .
$$

This is a direct consequence of the Calderón-Vaillancourt theorem using (2-8) with $q=2$, the inclusions in (2-20), and (2-21). In the "gauge" defined by $d G$, the latter gives

$$
\left\|e^{-\gamma_{n} r} \widehat{O p}_{\iota}(a) e^{\gamma_{n} r}\right\|_{L^{2}(\mathcal{M}, d G) \rightarrow L^{2}(\mathcal{M}, d G)} \lesssim 1, \quad h \in(0,1] .
$$

Working with the measure $\widehat{d G}$ is to this extent more natural and avoids to deal with exponential weights. 
We now describe the typical symbols we shall use in this paper. Using (2-17), (2-18), (2-19) and (2-21), we can write

$$
h^{2} P=\sum_{\iota \in \mathscr{I}} \widehat{O P}_{\iota}\left(\left(\kappa \otimes \kappa_{\iota}\right) \times\left(p_{\iota}+h p_{\iota, 1}+h^{2} p_{\iota, 0}\right)\right), \quad r>R_{\mathscr{K}}+2,
$$

using (2-13), (2-14) and (2-15). One observes that the symbols involved in (2-28) are of the form

$$
a_{\iota}(r, \theta, \rho, \eta)=\tilde{a}_{\iota}\left(r, \theta, \rho, e^{-r} \eta\right),
$$

with $\tilde{a}_{\iota} \in S^{2}\left(\mathbb{R}^{n} \times \mathbb{R}^{n}\right)$. It will turn out that the functional calculus of $h^{2} P$ (or $h^{2} \Delta_{G}$ ) will involve more generally symbols of this form with $a_{\iota} \in S^{-\infty}\left(\mathbb{R}^{n} \times \mathbb{R}^{n}\right)$. For instance, if $f \in C_{0}^{\infty}(\mathbb{R})$, the semiclassical principal symbol of $f\left(h^{2} P\right)$ or $f\left(-h^{2} \Delta_{G}\right)$ will be

$$
f\left(\rho^{2}+q_{\iota}\left(r, \theta, e^{-r} \eta\right)\right)
$$

which, once multiplied by the cutoff $\kappa \otimes \kappa_{\iota}$, is of the form (2-29) with $\tilde{a}_{\iota} \in S^{-\infty}\left(\mathbb{R}^{n} \times \mathbb{R}^{n}\right)$. This type of symbols is the model of functions described in Definition 2.2 below. To state this definition, we introduce the notation

$$
D_{\text {hyp }}^{j \alpha k \beta}:=e^{r|\beta|} \partial_{\eta}^{\beta} \partial_{r}^{j} \partial_{\theta}^{\alpha} \partial_{\rho}^{k}
$$

for all $j, k \in \mathbb{N}_{0}$ and $\alpha, \beta \in \mathbb{N}_{0}^{n-1}$.

Definition 2.2. Given an open set $\Omega \subset T^{*} \mathbb{R}_{+}^{n}=(0,+\infty)_{r} \times \mathbb{R}_{\theta}^{n-1} \times \mathbb{R}_{\rho} \times \mathbb{R}_{\theta}^{n-1}$, we define

$$
\mathscr{B}_{\text {hyp }}(\Omega)=\left\{a \in C^{\infty}(\Omega): D_{\text {hyp }}^{j \alpha k \beta} a \in L^{\infty}(\Omega) \text { for all } j, k \in \mathbb{N}_{0}, \alpha, \beta \in \mathbb{N}_{0}^{n-1}\right\}
$$

and

$$
\mathscr{S}_{\text {hyp }}(\Omega)=\left\{a \in C^{\infty}\left(\mathbb{R}^{2 n}\right): \operatorname{supp}(a) \subset \Omega \text { and } a \in \mathscr{B}_{\text {hyp }}(\Omega)\right\} .
$$

A family $\left(a_{v}\right)_{v \in \Lambda}$ is bounded in $\mathscr{B}_{\mathrm{hyp}}(\Omega)$ if $\left(D_{\text {hyp }}^{j \alpha k \beta} a_{v}\right)_{v \in \Lambda}$ is bounded in $L^{\infty}(\Omega)$ for all $j, k, \alpha, \beta$.

Note that considering $\Omega \subset T^{*} \mathbb{R}_{+}^{n}$ is not necessary but, since we shall work only in the region where $r \gg 1$, this will be sufficient.

Example 2.3. Consider the following diffeomorphism from $\mathbb{R}^{2 n}$ onto itself

$$
F_{\text {hyp }}:(r, \theta, \rho, \eta) \mapsto\left(r, \theta, \rho, e^{-r} \eta\right) .
$$

If $a_{\iota} \in S^{0}\left(\mathbb{R}^{n} \times \mathbb{R}^{n}\right)$ is supported in $F_{\text {hyp }}(\Omega)$, with $\Omega \subset T^{*} \mathbb{R}_{+}^{n}$, then (2-29) belongs to $\mathscr{S}_{\text {hyp }}(\Omega)$.

Proof. We only need to check that (2-29) belongs to $\mathscr{B}_{\text {hyp }}(\Omega)$. We have

$$
\partial_{r}\left(\tilde{a}_{\iota}\left(r, \theta, \rho, e^{-r} \eta\right)\right)=\left(\partial_{r} \tilde{a}_{\iota}\right)\left(r, \theta, \rho, e^{-r} \eta\right)-e^{-r} \eta \cdot\left(\partial_{\xi} \tilde{a}_{\iota}\right)(r, \theta, \rho, \xi)_{\mid \xi=e^{-r} \eta},
$$

which is bounded since $\xi \cdot \partial_{\xi} a_{\iota}$ is bounded. Similarly

$$
e^{r} \partial_{\eta}\left(\tilde{a}_{\iota}\left(r, \theta, \rho, e^{-r} \eta\right)\right)=\left(\partial_{\xi} \tilde{a}_{\iota}\right)(r, \theta, \rho, \xi)_{\mid \xi=e^{-r} \eta},
$$

is bounded too. Derivatives with respect to $\rho, \theta$ are harmless and higher-order derivatives in $r, \eta$ are treated similarly.

The next lemma gives a characterization of functions in $\mathscr{B}_{\mathrm{hyp}}(\Omega)$. 
Lemma 2.4. Let $\Omega \subset T^{*} \mathbb{R}_{+}^{n}$ be an open subset and assume that

$$
F_{\text {hyp }}(\Omega) \subset \mathbb{R}_{+}^{n} \times B, \quad \text { with B bounded } .
$$

A function $a \in C^{\infty}(\Omega)$ is of the form

$$
a(r, \theta, \rho, \eta)=\tilde{a}\left(r, \theta, \rho, e^{-r} \eta\right), \quad \text { with } \tilde{a} \in C_{b}^{\infty}\left(F_{\mathrm{hyp}}(\Omega)\right),
$$

if and only if

$$
D_{\text {hyp }}^{j \alpha k \beta} a \in L^{\infty}(\Omega) \quad \text { for all } j, k, \alpha, \beta .
$$

Here $C_{b}^{\infty}(\Omega)$ and $C_{b}^{\infty}\left(F_{\mathrm{hyp}}(\Omega)\right)$ are spaces of smooth functions bounded with all derivatives bounded on $\Omega$ and $F_{\text {hyp }}(\Omega)$, respectively.

Proof. That (2-33) implies (2-34) is proved in the same way as Example 2.3: the boundedness of $\xi \cdot \partial_{\xi} \tilde{a}$ follows from the boundedness of $\xi=e^{-r} \eta$ in $F_{\text {hyp }}(\Omega)$ by (2-32) and the fact that $\tilde{a} \in C_{b}^{\infty}\left(F_{\text {hyp }}(\Omega)\right)$. Conversely, one checks by induction that

$$
\tilde{a}(r, \theta, \rho, \xi):=a\left(r, \theta, \rho, e^{r} \xi\right),
$$

belongs to $C_{b}^{\infty}\left(F_{\text {hyp }}(\Omega)\right)$, using again the boundedness of $\xi$ on $F_{\text {hyp }}(\Omega)$.

Example 2.5. For all $f \in C_{0}^{\infty}\left(\mathbb{R}^{n}\right)$, all $R>R_{\mathscr{K}}$ and all $V \Subset \Psi_{\iota}\left(U_{\iota}\right)$, (2-30) satisfies the conditions of this lemma with $\Omega=(R,+\infty) \times V \times \mathbb{R}^{n}$.

Proof. By (1-8), there exists $C>1$ such that

$$
C^{-1}|\xi|^{2} \lesssim q_{\iota}(r, \theta, \xi) \lesssim C|\xi|^{2} \quad \text { for } r>R, \theta \in V, \xi \in \mathbb{R}^{n-1},
$$

and, using the notation (2-13),

$$
\left|\partial_{r}^{j} \partial_{\theta}^{\alpha} g^{k l}(r, \theta)\right| \leq C_{j k} \quad \text { for } r>R, \theta \in V .
$$

Therefore, (2-35) and the compact support of $f$ ensure that $e^{-r} \eta$ and $\rho$ are bounded, hence that (2-32) holds on the support of (2-30). Then, (2-36) implies that $f\left(\rho^{2}+q_{\iota}(r, \theta, \xi)\right)$ belongs to $C_{b}^{\infty}\left(F_{\text {hyp }}(\Omega)\right)$ (notice that here $\left.F_{\text {hyp }}(\Omega)=(R,+\infty) \times V \times \mathbb{R}^{n}\right)$.

We conclude this subsection with the following useful remarks. If $a, b \in \mathscr{S}_{\text {hyp }}(\Omega)$ for some $\Omega$ (such $a, b$ satisfy (2-23)), we have the composition rule

$$
\widehat{O P}_{\iota}(a) \widehat{O P}_{\iota}(b)=\widehat{O P}_{\iota}((a \# b)(h)),
$$

if $(a \# b)(h)$ denotes the full symbol of $a\left(r, \theta, h D_{r}, h D_{\theta}\right) b\left(r, \theta, h D_{r}, h D_{\theta}\right)$. In particular all the terms of the expansion of $(a \# b)(h)$ belong to $\mathscr{Y}_{\text {hyp }}(\Omega)$ and are supported in $\operatorname{supp}(a) \cap \operatorname{supp}(b)$. Similarly, for all $N \geq 0$, we have

$$
\widehat{O p}_{\iota}(a)^{*}=\widehat{O p}_{\iota}\left(a_{0}^{*}+\cdots+h^{N} a_{N}^{*}\right)+h^{N+1} R_{N}(a, h)
$$

with $a_{0}^{*}, \ldots, a_{N}^{*} \in \mathscr{S}_{\text {hyp }}(\Omega)$ supported in $\operatorname{supp}(a)$ and $\left\|R_{N}(a, h)\right\|_{L^{2}(\mathcal{M}, \widehat{d G}) \rightarrow L^{2}(\mathcal{M}, \widehat{d G})} \lesssim 1$ for $h \in(0,1]$. 
2C. The functional calculus. In Proposition 2.7 below, we give two pseudodifferential approximations of $f\left(h^{2} P\right)$ near infinity of $\mathcal{M}$, when $f \in C_{0}^{\infty}(\mathbb{R})$. The first approximation, namely (2-43), is given in terms of the "quantization" $\widehat{O P}$, defined in the previous subsection. This is the one we shall mostly use in this paper. However, at some crucial points, we shall need another approximation, (2-44), which uses properly supported $\Psi$ DOs.

To define such properly supported operators, we need a function

$$
\zeta \in C_{0}^{\infty}\left(\mathbb{R}^{n}\right), \quad \zeta \equiv 1 \text { near } 0, \quad \operatorname{supp}(\zeta) \text { small enough, }
$$

which will basically be used as a cutoff near the diagonal. The smallness of the support will be fixed in the following definition.

Definition 2.6. For $\iota \in \mathscr{I}$, all $h \in(0,1]$ and all symbol $a$ satisfying (2-23), we define

$$
O p_{\iota, \mathrm{pr}}(a): C_{0}^{\infty}(\mathcal{M}) \rightarrow C^{\infty}(\mathcal{M})
$$

as the unique operator with kernel supported in $U_{\iota}^{2}$ and such that the kernel of $\Psi_{\iota}^{*} \widehat{O p}_{\iota}(a) \Psi_{l *}$ is

$$
(2 \pi h)^{-n} \iint e^{\frac{i}{h}\left(r-r^{\prime}\right) \rho+\frac{i}{h}\left(\theta-\theta^{\prime}\right) \cdot \eta} a(r, \theta, \rho, \eta) d \rho d \eta \zeta\left(r-r^{\prime}, \theta-\theta^{\prime}\right) .
$$

The advantage of choosing the support of $\zeta$ small enough is that, using (2-23), we can assume that, on the support of (2-39), $r^{\prime}$ belongs to a neighborhood of $\left[R_{\mathscr{K}}+1,+\infty\right)$ and $\theta^{\prime}$ belongs to a neighborhood of $V_{\iota}^{\prime}$. For instance, we may assume that $r^{\prime} \in \tilde{\kappa}^{-1}(1)$ and $\theta^{\prime} \in \tilde{\kappa}_{\iota}^{-1}(1)$ so that we can put a factor $\tilde{\kappa}\left(r^{\prime}\right) \tilde{\kappa}_{\iota}\left(\theta^{\prime}\right)$ for free to the right-hand side of (2-39). The latter implies, using (2-8), (2-25), and (2-39), the standard off-diagonal fast decay of kernels of $\Psi$ DOs and the Calderón-Vaillancourt theorem stating that, for all $a \in C_{b}^{\infty}\left(\mathbb{R}^{2 n}\right)$ satisfying (2-23) and all $N \in \mathbb{N}_{0}$, we have

$$
\left\|\widehat{O P}_{\iota}(a)-O p_{\iota, \mathrm{pr}}(a)\right\|_{L^{2}(\mathcal{M}, \widehat{d G}) \rightarrow L^{2}(\mathcal{M}, \widehat{d G})} \lesssim h^{N}, \quad h \in(0,1] .
$$

This shows that, up to remainders of size $h^{\infty}, \widehat{O p}_{\iota}(a)$ and $O p_{\iota, \mathrm{pr}}(a)$ coincide as bounded operators on $L^{2}(M, \widehat{d G})$. Under the same assumptions on $a$, we also have

$$
\left\|O p_{\iota, \mathrm{pr}}(a)\right\|_{L^{2}(\mathcal{M}, d G) \rightarrow L^{2}(\mathcal{M}, d G)} \lesssim 1, \quad h \in(0,1]
$$

which is a first difference with $\widehat{O P}_{\iota}(a)$ for which we have only (2-27) in general. The estimate (2-41) is equivalent to the uniform boundedness (with respect to $h \in(0,1])$ of $e^{\gamma_{n} r} O p_{\iota, p r}(a) e^{-\gamma_{n} r}$ on $L^{2}(M, \widehat{d G})$. The latter is obtained similarly to (2-26), using the Calderón-Vaillancourt theorem, for we only have to consider the kernel obtained by multiplying (2-39) by $e^{\gamma_{n}\left(r-r^{\prime}\right)}$, which is bounded (as well as its derivatives) on the support of $\zeta\left(r-r^{\prime}, \theta-\theta^{\prime}\right)$.

In other words, (2-41) can be interpreted as a boundedness result between (exponentially) weighted $L^{2}$ spaces. Similar properties holds for $L^{q}$ spaces (under suitable assumptions on the symbol $a$ ) and they are the main reason for considering properly supported operators. In particular, they lead to following proposition, where we collect the estimates we shall need in this paper. We refer to [Bouclet 2007] for the proof. 
Proposition 2.7. Let $f \in C_{0}^{\infty}(\mathbb{R})$ and let $I \Subset(0,+\infty)$ be an open interval containing $\operatorname{supp}(f)$. Let $\chi_{\mathscr{K}} \in C_{0}^{\infty}(M)$ and $R>R_{\mathscr{K}}+1$ be such that

$$
\chi_{\mathscr{K}}(m)=1 \quad \text { if } r(m) \leq R+1 .
$$

Then, for all $N \geq 0$ and all $\iota \in \mathscr{I}$, we can find symbols

$$
a_{\iota, 0}(f), \ldots, a_{\iota, N}(f) \in \mathscr{I}_{\text {hyp }}\left((R,+\infty) \times V_{\iota} \times \mathbb{R}^{n} \cap p_{\iota}^{-1}(I)\right)
$$

(where $p_{\iota}$ is the principal symbol of $P$ in the chart $U_{\iota}$ ) such that, if we set

$$
a_{\iota}^{(N)}(f, h)=a_{\iota, 0}(f)+h a_{\iota, 1}(f)+\cdots+h^{N} a_{\iota, N}(f),
$$

we have

$$
\begin{aligned}
& \left(1-\chi_{\mathscr{K}}\right) f\left(h^{2} P\right)=\sum_{\iota \in \mathscr{I}} \widehat{O p}_{\iota}\left(a_{\iota}^{(N)}(f, h)\right)+h^{N+1} \widehat{R}_{N}(f, h), \\
& \left(1-\chi_{\mathscr{Y}}\right) f\left(h^{2} P\right)=\sum_{\iota \in \mathscr{I}} O p_{\iota, \mathrm{pr}}\left(a_{\iota}^{(N)}(f, h)\right)+h^{N+1} R_{N, \mathrm{pr}}(f, h),
\end{aligned}
$$

where, for each $q \in[2, \infty]$,

$$
\left\|e^{-\gamma_{n} r} R_{N, \operatorname{pr}}(f, h)\right\|_{L^{2}(\mathcal{M}, \widehat{d G}) \rightarrow L^{q}(\mathcal{M}, d G)} \lesssim h^{-n\left(\frac{1}{2}-\frac{1}{q}\right)} \quad \text { for } h \in(0,1],
$$

and

$$
\left\|\widehat{R}_{N}(f, h)\right\|_{L^{2}(\mathcal{M}, \widehat{d G}) \rightarrow L^{2}(\mathcal{M}, \widehat{d G})} \lesssim 1 \quad \text { for } h \in(0,1] .
$$

In addition, for all $\iota \in \mathscr{I}$ and all $q \in[2, \infty]$, we have

$$
\left\|e^{-\gamma_{n} r} O p_{\iota, \operatorname{pr}}\left(a_{\iota}^{(N)}(f, h)\right)\right\|_{L^{2}(M, \widehat{d G}) \rightarrow L^{q}(\mathcal{M}, d G)} \lesssim h^{-n\left(\frac{1}{2}-\frac{1}{q}\right)} \quad \text { for } h \in(0,1],
$$

and, for all $q \in[1, \infty]$ and all $\gamma \in \mathbb{R}$,

$$
\left\|e^{-\gamma r} O p_{\iota, \mathrm{pr}}\left(a_{\iota}^{(N)}(f, h)\right) e^{\gamma r}\right\|_{L^{q}(\mathcal{M}, \widehat{d G}) \rightarrow L^{q}(\mathcal{M}, \widehat{d G})} \lesssim 1 \quad \text { for } h \in(0,1] .
$$

In this proposition, as well as in further definitions or propositions, the interval $I$ can be considered as a semiclassical energy window, in the sense that the principal symbol of $h^{2} P$ will live in $I$. In the sequel, $I$ will be more explicitly of the form $\left(\frac{1}{4}, 4\right)$ or $\left(\frac{1}{4}-\varepsilon, 4+\varepsilon\right)$; see for instance $(2-54)$.

To make (2-42) more explicit, let us quote for instance that

$$
a_{\iota, 0}(f)(r, \theta, \rho, \eta)=\kappa(r) \kappa_{\iota}(\theta) f\left(\rho^{2}+q_{\iota}\left(r, \theta, e^{-r} \eta\right)\right) \times\left(1-\chi_{\mathscr{K}}\right)\left(\Psi_{\iota}^{-1}(r, \theta)\right) .
$$

More generally, (2-42) and Lemma 2.4 show that $a_{\iota, 0}(f), \ldots, a_{\iota, N}(f)$ are of the form (2-29), with $\tilde{a}_{\iota}(r, \theta, \rho, \xi)$ compactly supported with respect to $(\rho, \xi)$.

The estimate (2-48) basically means that $O p_{\iota, \mathrm{pr}}\left(a_{\iota}^{(N)}(f, h)\right)$ preserves all $L^{q}$ spaces with any exponential weights. In particular, since $L^{q}(\mathcal{M}, d G)=e^{-\gamma_{n} r / q} L^{q}(\mathcal{M}, \widehat{d G})$, replacing $\widehat{d G}$ by $d G$ in $(2-48)$ would give a completely equivalent statement. This estimate is the main reason for introducing properly supported operators. Of course, (2-48) holds for other symbols than those involved in the functional calculus of $P$. We have more generally (see [Bouclet 2007]) for all $\gamma \in \mathbb{R}$,

$$
\left\|e^{-\gamma r} O p_{\iota, \mathrm{pr}}\left(a_{\iota}\right) e^{\gamma r}\right\|_{L^{q}(\mathcal{M}, \widehat{d G}) \rightarrow L^{q}(\mathcal{M}, \widehat{d G})} \lesssim 1 \quad \text { for } h \in(0,1],
$$


for any $q \in[1, \infty]$ and any

$$
a_{\iota} \in \mathscr{Y}_{\text {hyp }}\left(\left(R_{\mathscr{K}}+1,+\infty\right) \times V_{\iota}^{\prime} \times \mathbb{R}^{n} \cap p_{\iota}^{-1}\left(I^{\prime}\right)\right),
$$

provided $I^{\prime}$ is bounded.

By the unitary equivalence of $P$ and $-\Delta_{G}-\gamma_{n}^{2}$, we would get a very similar pseudodifferential expansion for $f\left(-h^{2} \Delta_{G}\right)$. (Here we have only described $\left(1-\chi_{\mathscr{K}}\right) f\left(h^{2} P\right)$ since this will be sufficient for our present purpose, but of course there is a completely analogous result for the compactly supported part $\chi_{\mathscr{K}} f\left(h^{2} P\right)$; see [Bouclet 2007].) Such an approximation of $f\left(-h^{2} \Delta_{G}\right)$ was used in [Bouclet 2010] to prove the next two propositions.

Proposition 2.8. Consider a dyadic partition of unit

$$
1=f_{0}(\lambda)+\sum_{k \geq 0} f\left(2^{-k} \lambda\right),
$$

for $\lambda$ in a neighborhood of $[0,+\infty)$, with

$$
f_{0} \in C_{0}^{\infty}(\mathbb{R}), \quad f \in C_{0}^{\infty}\left(\left[\frac{1}{4}, 4\right]\right) .
$$

Then, for all $\chi \in C_{0}^{\infty}(\mathcal{M})$ and all $q \in[2, \infty)$, we have

$$
\|(1-\chi) u\|_{L^{q}(\mathcal{M}, d G)} \lesssim\left(\sum_{\substack{h^{2}=2^{-k} \\ k \geq 0}}\left\|(1-\chi) f\left(-h^{2} \Delta_{G}\right) u\right\|_{L^{q}(\mathcal{M}, d G)}^{2}\right)^{1 / 2}+\|u\|_{L^{2}(\mathcal{M}, d G)} .
$$

This proposition leads to the following classical reduction.

Proposition 2.9. Let $\chi \in C_{0}^{\infty}(\mathcal{M})$ and $(p, q)$ be an admissible pair. Then (1-10) holds true if and only if there exists $C$ such that

$$
\left\|(1-\chi) e^{i t \Delta_{G}} f\left(-h^{2} \Delta_{G}\right) u_{0}\right\|_{L^{p}\left([0,1] ; L^{q}(M, d G)\right)} \leq C\left\|u_{0}\right\|_{L^{2}(M, d G)},
$$

for all $h \in(0,1]$ and $u_{0} \in C_{0}^{\infty}(M)$.

This result is essentially well known and proved in [Bouclet 2010] for a class of noncompact manifolds. We simply recall here that the $L^{q} \rightarrow L^{q}$ boundedness of the spectral cutoffs $f\left(-h^{2} \Delta_{G}\right)$ is not necessary to prove this result, although the latter slightly simplifies the proof when it is available.

2D. Outgoing and incoming areas. Propositions 2.7 and 2.9 lead to a microlocalization of Theorem 1.2: as we shall see more precisely in Section 2E, they allow to reduce the proof of (1-10) to the same estimate in which $1-\chi$ is replaced by $h-\Psi D O s$. But this microlocalization, i.e., the support of the symbols in (2-42), is still too rough to simplify the proof of Theorem 1.2 in a significant way. The purpose of this subsection is to describe convenient regions which will refine this localization.

Definition 2.10. Fix $\iota \in \mathscr{I}$. For $R>R_{\mathscr{K}}+1$, an open subset $V \Subset V_{\iota}^{\prime}$ (see (2-21)), an open interval $I \Subset(0,+\infty)$ and $\sigma \in(-1,1)$, we define

$$
\Gamma_{\iota}^{ \pm}(R, V, I, \sigma)=\left\{(r, \theta, \rho, \eta) \in \mathbb{R}^{2 n}: r>R, \theta \in V, p_{\iota} \in I, \pm \rho>-\sigma p_{\iota}^{1 / 2}\right\},
$$

where $p_{\iota}$ is the principal symbol of $P$ in the chart $U_{\iota}$ given by $(2-14)$. The open set $\Gamma_{\iota}^{+}(R, V, I, \sigma)$ is called an outgoing area, and $\Gamma_{\iota}^{-}(R, V, I, \sigma)$ an incoming area. 
We note in passing that, except from the localization in $\theta$, these areas are defined using only the variable $r$, its dual $\rho$ and the principal symbol of $P$. In particular, up to the choice of the coordinate $r$, the conditions $r>R, p_{\iota} \in I$ and $\pm \rho>-\sigma p_{\iota}^{1 / 2}$ define invariant subsets of $T^{*} M$. However the whole analysis in this paper will be localized in charts and we will not use this invariance property.

Let us record some useful properties of outgoing/incoming areas. First, they decrease with respect to $V, I, \sigma$ and $R^{-1}$ :

$$
R_{1} \geq R_{2}, \quad V_{1} \subset V_{2}, \quad I_{1} \subset I_{2}, \quad \sigma_{1} \leq \sigma_{2} \quad \Longrightarrow \quad \Gamma_{\iota}^{ \pm}\left(R_{1}, V_{1}, I_{1}, \sigma_{1}\right) \subset \Gamma_{\iota}^{ \pm}\left(R_{2}, V_{2}, I_{2}, \sigma_{2}\right) .
$$

Second, we have

$$
\Gamma_{\iota}^{+}\left(R, V, I, \frac{1}{2}\right) \cup \Gamma_{\iota}^{-}\left(R, V, I, \frac{1}{2}\right)=(R,+\infty) \times V \times \mathbb{R}^{n} \cap p_{\iota}^{-1}(I) .
$$

Here we have chosen $\sigma=\frac{1}{2}$ but any $\sigma \in(0,1)$ would work as well.

We will use the following elementary property, proved later as part (ii) of Proposition 4.1.

Proposition 2.11. Any symbol $a \in \mathscr{Y}_{\text {hyp }}\left((R,+\infty) \times V \times \mathbb{R}^{n} \cap p_{\iota}^{-1}(I)\right)$ can be written as

$$
a=a^{+}+a^{-}, \quad \text { with } a^{ \pm} \in \mathscr{S}_{\text {hyp }}\left(\Gamma_{\iota}^{ \pm}\left(R, V, I, \frac{1}{2}\right)\right) .
$$

This splitting into outgoing/incoming areas was sufficient to use the Isozaki-Kiada parametrix in the asymptotically Euclidean case; in the AH case, we will only be able to construct this parametrix in much smaller areas, called strongly outgoing/incoming areas, which we now introduce.

We first describe briefly the meaning of such areas, say in the outgoing case. Basically, being in an outgoing area means that $\rho$ is not too close to $-p^{1 / 2}$; the aim of strongly outgoing areas is to guarantee that $\rho$ is very close to $p^{1 / 2}$, which is of course a much stronger restriction. This amounts essentially to chose $\sigma$ close to -1 in the definition of outgoing areas. We will measure this closeness in term of a small parameter $\varepsilon$. It will actually be convenient to have the other parameters, namely $R, V, I$, depending also on $\varepsilon$, so we introduce

$$
R(\varepsilon)=1 / \varepsilon, \quad V_{\iota, \varepsilon}=\left\{\theta \in \mathbb{R}^{n-1}: \operatorname{dist}\left(\theta, V_{\iota}\right)<\varepsilon^{2}\right\}, \quad I(\varepsilon)=\left(\frac{1}{4}-\varepsilon, 4+\varepsilon\right),
$$

where we recall that $V_{\iota}$ is defined in (2-21).

Definition 2.12. For all $\varepsilon>0$ small enough, we set

$$
\Gamma_{\iota, \mathrm{s}}^{ \pm}(\varepsilon):=\Gamma_{\iota}^{ \pm}\left(R(\varepsilon), V_{\iota, \varepsilon}, I(\varepsilon), \varepsilon^{2}-1\right) .
$$

The open set $\Gamma_{\iota, \mathrm{s}}^{+}(\varepsilon)$ is called a strongly outgoing area, and $\Gamma_{\iota, \mathrm{s}}^{-}(\varepsilon)$ a strong incoming area.

The main interest of such areas is to ensure that $e^{-r}|\eta|$ is small if $\varepsilon$ is small. Indeed, if $q \in[0,+\infty)$ and $-1<\sigma<0$, we have the equivalence

$$
\pm \rho>-\sigma\left(\rho^{2}+q\right)^{1 / 2} \Longleftrightarrow \pm \rho>0 \text { and } q<\sigma^{-2}\left(1-\sigma^{2}\right) \rho^{2} .
$$

Therefore, there exists $C$ such that, for all $\varepsilon$ small enough and $(r, \theta, \rho, \eta) \in \Gamma_{\iota, \mathrm{s}}^{ \pm}(\varepsilon)$,

$$
q_{\iota}\left(r, \theta, e^{-r} \eta\right) \leq C \varepsilon^{2},
$$

which, by (2-35), is equivalent to

$$
\left|e^{-r} \eta\right| \lesssim \varepsilon
$$


Note also that, by (2-52), strongly outgoing/incoming areas decrease with $\varepsilon$.

We now quote a result that motivates, at least partially, the introduction of strongly outgoing/incoming areas. Its proof is given in Section 4A.

Denote by $\Phi_{\iota}^{t}$ the Hamiltonian flow of $p_{\iota}$. This is of course the geodesic flow written in the chart $\Psi_{\iota}\left(u_{\iota}\right) \times \mathbb{R}^{n}$ of $T^{*} M$.

Proposition 2.13. Fix $\sigma \in(-1,1)$. There exists $R_{\sigma}^{\prime}>0$ such that for all $R \geq R_{\sigma}^{\prime}$ and all $\varepsilon>0$ small enough, there exists $t_{R, \varepsilon} \geq 0$ such that

$$
\Phi_{\iota}^{t}\left(\Gamma_{\iota}^{ \pm}\left(R, V_{\iota},\left(\frac{1}{4}-\varepsilon, 4+\varepsilon\right), \sigma\right)\right) \subset \Gamma_{\iota, \mathrm{s}}^{ \pm}(\varepsilon) \quad \text { if } \pm t \geq t_{R, \varepsilon}
$$

In particular, for all $\varepsilon>0$ small enough, there exists $T_{\varepsilon}>0$ such that

$$
\Phi_{\iota}^{t}\left(\Gamma_{\iota}^{ \pm}\left(R(\varepsilon), V_{\iota}, I(\varepsilon), \sigma\right)\right) \subset \Gamma_{\iota, \mathrm{s}}^{ \pm}(\varepsilon) \quad \text { if } \pm t \geq T_{\varepsilon} .
$$

Note that, since $p_{\iota}$ is only defined in the chart $\Psi_{\iota}\left(U_{\iota}\right) \times \mathbb{R}^{n}$, its flow is not complete. We shall however see in Section 3 that, for any initial data $(r, \theta, \rho, \eta) \in \Gamma_{\iota}^{ \pm}\left(R(\varepsilon), V_{\iota}, I, \sigma\right), \Phi_{\iota}^{t}(r, \theta, \rho, \eta)$ is well defined for all $\pm t \geq 0$; that is, $\Phi_{\iota}^{t}(r, \theta, \rho, \eta) \in \Psi_{\iota}\left(U_{\iota}\right) \times \mathbb{R}^{n}$ for all $\pm t \geq 0$.

Proposition 2.13 essentially states that the forward flow sends outgoing areas into strongly outgoing areas in finite time, and likewise the backward flow sends incoming areas into strongly incoming ones. This will be interesting for the proof of Proposition 2.24.

The last type of region we need to consider are the intermediate areas. They should have two properties: firstly they should essentially cover the complement of strongly outgoing/incoming areas in outgoing/incoming areas and, secondly, be small enough.

To define them we need the following. For all $\varepsilon>0$ and all $\delta>0$, we can find $L+1$ real numbers, $\sigma_{0}, \ldots, \sigma_{L}$,

$$
(\varepsilon / 2)^{2}-1=\sigma_{0}<\sigma_{1}<\cdots<\sigma_{L}=\frac{1}{2}
$$

such that

and

$$
\left((\varepsilon / 2)^{2}-1, \frac{1}{2}\right)=\bigcup_{l=1}^{L-1}\left(\sigma_{l-1}, \sigma_{l+1}\right)
$$

$$
\left|\sigma_{l+1}-\sigma_{l-1}\right| \leq \delta
$$

Note that the intervals overlap in (2-59), since $\left(\sigma_{l-1}, \sigma_{l+1}\right)$ always contains $\sigma_{l}$.

Definition 2.14. The intermediate outgoing and incoming areas associated to the cover (2-59) are

$$
\Gamma_{\iota, \text { inter }}^{ \pm}(\varepsilon, \delta ; l):=\left\{(r, \theta, \rho, \eta) \in \mathbb{R}^{2 n}: r>R(\varepsilon), \theta \in V_{\iota}, p_{\iota} \in I(\varepsilon), \pm \rho / p_{\iota}^{1 / 2} \in\left(-\sigma_{l+1},-\sigma_{l-1}\right)\right\},
$$

for $1 \leq l \leq L-1$.

Notice that, by definition,

$$
\Gamma_{\iota, \text { inter }}^{ \pm}(\varepsilon, \delta ; l) \subset \Gamma_{\iota}^{ \pm}\left(R(\varepsilon), V_{\iota}, I(\varepsilon), \frac{1}{2}\right) .
$$

In the notation, we only specify the parameters which are relevant for our analysis, namely $\varepsilon, \delta$, but, of course, intermediate areas depend on the choice of $\sigma_{1}, \ldots, \sigma_{L}$. Here $\delta$ measures the smallness and Proposition 2.16 below will explain how to choose this parameter. 
Proposition 2.15. Fix $\varepsilon>0$ small enough, $\delta>0$ and $\sigma_{0}, \ldots, \sigma_{L}$ satisfying (2-58), (2-59) and (2-60). Then, any symbol

$$
a^{ \pm} \in \mathscr{S}_{\text {hyp }}\left(\Gamma_{\iota}^{ \pm}\left(R(\varepsilon), V_{\iota}, I(\varepsilon), \frac{1}{2}\right)\right)
$$

can be written as

$$
a^{ \pm}=a_{\mathrm{s}}^{ \pm}+a_{1, \text { inter }}^{ \pm}+\cdots+a_{L-1, \text { inter }}^{ \pm},
$$

with $a_{\mathrm{s}}^{ \pm} \in \mathscr{Y}_{\text {hyp }}\left(\Gamma_{l, \mathrm{~s}}^{ \pm}(\varepsilon)\right)$ and $a_{l, \text { inter }}^{ \pm} \in \mathscr{S}_{\text {hyp }}\left(\Gamma_{l, \text { inter }}^{ \pm}(\varepsilon, \delta ; l)\right)$.

The proof is given in Section 4A.

We conclude this subsection with the following proposition which will be crucial for the proof of Theorem 1.2 and motivates the introduction of intermediate areas. The proof is given in Section 4A.

Proposition 2.16. Fix $\underline{t}>0$. Then, for all $\varepsilon>0$ small enough, we can find $\delta>0$ small enough such that, for any choice of $\sigma_{0}, \ldots, \sigma_{L}$ satisfying (2-58), (2-59) and (2-60), we have, for all $1 \leq l \leq L-1$,

$$
\Phi_{\iota}^{t}\left(\Gamma_{l, \text { inter }}^{ \pm}(\varepsilon, \delta ; l)\right) \cap \Gamma_{l, \text { inter }}^{ \pm}(\varepsilon, \delta ; l)=\varnothing,
$$

provided that

$$
\pm t \geq \underline{t} .
$$

2E. The main steps of the proof of Theorem 1.2. We already know from Proposition 2.9 that we only have to find $\chi \in C_{0}^{\infty}(\mathcal{M})$ such that (2-51) holds, which is equivalent to

$$
\left\|e^{-\gamma_{n} r}(1-\chi) f\left(h^{2} P\right) e^{-i t P} u_{0}\right\|_{L^{p}\left([0,1] ; L^{q}(\mathcal{M}, d G)\right)} \leq C\left\|u_{0}\right\|_{L^{2}(\mathcal{M}, \widehat{d G})},
$$

using the unitary map (2-9) and (2-11), (2-12).

Before choosing $\chi$, we introduce the following operators. Choose a cutoff $\tilde{f} \in C_{0}^{\infty}((0,+\infty))$ such that $\tilde{f} f=f$.

Lemma 2.17. For all $\chi \in C_{0}^{\infty}(\mathcal{M})$, we can write

$$
(1-\chi) \tilde{f}\left(h^{2} P\right)=(1-\chi) A_{\mathrm{pr}}(h)+R(h)
$$

with $R(h)$ satisfying, for all $q \in[2, \infty]$,

$$
\left\|e^{-\gamma_{n} r} R(h)\right\|_{L^{2}(\mathcal{M}, \widehat{d G}) \rightarrow L^{q}(\mathcal{M}, d G)} \lesssim 1,
$$

and $A_{\mathrm{pr}}(h)$ such that, for all $q \in[2, \infty]$,

$$
\begin{aligned}
\left\|e^{-\gamma_{n} r} A_{\mathrm{pr}}(h)\right\|_{L^{2}(\mathcal{M}, \widehat{d G}) \rightarrow L^{q}(\mathcal{M}, d G)} & \lesssim h^{-n\left(\frac{1}{2}-\frac{1}{q}\right)}, \\
\left\|e^{-\gamma_{n} r} A_{\mathrm{pr}}(h) e^{\gamma_{n} r}\right\|_{L^{\infty}(\mathcal{M}, d G) \rightarrow L^{\infty}(\mathcal{M}, d G)} & \lesssim 1, \\
\left\|A_{\mathrm{pr}}(h)^{*} e^{-\gamma_{n} r}\right\|_{L^{1}(\mathcal{M}, \widehat{d G}) \rightarrow L^{2}(\mathcal{M}, \widehat{d G})} & \lesssim h^{-n / 2}, \\
\left\|e^{\gamma_{n} r} A_{\mathrm{pr}}(h)^{*} e^{-\gamma_{n} r}\right\|_{L^{1}(\mathcal{M}, \widehat{d G}) \rightarrow L^{1}(\mathcal{M}, \widehat{d G})} & \lesssim 1 .
\end{aligned}
$$

Proof. This is an immediate consequence of Proposition 2.7. Using (2-44), with $N$ such that $N+1 \geq n / 2$, we define $A_{\mathrm{pr}}(h)$ as the sum of the properly supported pseudodifferential operators. We thus have (2-64), (2-65) and (2-66). The estimates (2-67) and (2-68) are obtained by taking the adjoints (with $q=\infty$ in (2-65)) with respect to $\widehat{d G}$. 
Basically, the operators $e^{-\gamma_{n} r} A_{\mathrm{pr}}(h)$ and $A_{\mathrm{pr}}(h)^{*} e^{-\gamma_{n} r}$ will be used as "ghost cutoffs" to deal with remainder terms of parametrices which will be $O\left(h^{N}\right)$ in $\mathscr{L}\left(L^{2}(\mu, \widehat{d G})\right)$, using the Sobolev embeddings (2-65) and (2-67). They will be "transparent" for the principal terms of the parametrices by (2-66) and (2-68), which uses crucially that they are properly supported.

For $\varepsilon$ to be fixed below, we choose $\chi \in C_{0}^{\infty}(\mathcal{M})$ such that

$$
\chi \equiv 1 \text { for } r(m) \leq 3 \varepsilon^{-1} .
$$

This function will appear in Proposition 2.18 below only trough its support. More precisely, the proposition states that to prove (2-63) for such a $\chi$ (with $\varepsilon$ small enough), it is sufficient to prove the estimate (2-70) for a class of symbols supported where $r(m) \geq \varepsilon^{-1}$.

Proposition 2.18 (Microlocalization of Strichartz estimates). To prove (2-63), it is sufficient to show that, for some $\varepsilon$ small enough and all

$$
a_{\iota} \in \mathscr{Y}_{\text {hyp }}\left((R(\varepsilon),+\infty) \times V_{\iota} \times \mathbb{R}^{n} \cap p_{\iota}^{-1}(I(\varepsilon))\right),
$$

where we recall that $R(\varepsilon)=\varepsilon^{-1}$ and $I(\varepsilon)=\left(\frac{1}{4}-\varepsilon, 4+\varepsilon\right)$, we have

$$
\left\|e^{-\gamma_{n} r} A_{\mathrm{pr}}(h) \widehat{O p}_{\iota}\left(a_{l}\right) e^{-i t P} u_{0}\right\|_{L^{p}\left([0,1] ; L^{q}(\mathcal{M}, d G)\right)} \leq C\left\|u_{0}\right\|_{L^{2}(\mathcal{M}, \widehat{d G})},
$$

uniformly with respect to $h \in(0,1]$.

Proof. Choose $\chi_{0} \in C_{0}^{\infty}(\mathcal{M})$ such that

$$
\begin{array}{ll}
\chi_{0} \equiv 1 & \text { for } r(m) \leq \varepsilon^{-1}, \\
\chi_{0} \equiv 0 & \text { for } r(m) \geq 2 \varepsilon^{-1} .
\end{array}
$$

We then have $\left(1-\chi_{0}\right) \equiv 1$ near $\operatorname{supp}(1-\chi)$ so, by the proper support of the kernel of $A_{\text {pr }}(h)$, we also have

$$
(1-\chi) A_{\mathrm{pr}}(h)=(1-\chi) A_{\mathrm{pr}}(h)\left(1-\chi_{0}\right),
$$

at least for $\varepsilon$ small enough. The latter and (2-64) reduces the proof of (2-63) to the study of

$$
e^{-\gamma_{n} r} A_{\mathrm{pr}}(h)\left(1-\chi_{0}\right) f\left(h^{2} P\right) e^{-i t P} .
$$

By splitting $\left(1-\chi_{0}\right) f\left(h^{2} P\right)$ using (2-43) with $N+1 \geq n / 2$, we obtain the result using (2-46) and (2-65).

We now introduce a second small parameter $\delta>0$. By Propositions 2.11 and 2.15 , for all $\delta>0$, any $a_{\iota}$ satisfying (2-69) can be written as

$$
a_{\iota}=a_{\mathrm{s}}^{+}+a_{\mathrm{s}}^{-}+\sum_{l=1}^{L-1} a_{l, \text { inter }}^{+}+a_{l, \text { inter }}^{-}
$$

with

$$
a_{\mathrm{s}}^{ \pm} \in \mathscr{T}_{\mathrm{hyp}}\left(\Gamma_{l, \mathrm{~s}}^{ \pm}(\varepsilon)\right), \quad a_{l, \text { inter }}^{ \pm} \in \mathscr{S}_{\mathrm{hyp}}\left(\Gamma_{\iota, \text { inter }}^{ \pm}(\varepsilon, \delta ; l)\right)
$$


Proposition 2.19 (Reduction to microlocalized dispersion estimates). To prove (2-70), it is sufficient to show that, for some $\varepsilon$ and $\delta$ small enough, we have

$$
\begin{aligned}
\left\|e^{-\gamma_{n} r} A_{\mathrm{pr}}(h) \widehat{O P}_{\iota}\left(a_{\mathrm{s}}^{ \pm}\right) e^{-i t h P} \widehat{O p}_{\iota}\left(a_{\mathrm{s}}^{ \pm}\right)^{*} A_{\mathrm{pr}}(h)^{*} e^{-\gamma_{n} r}\right\|_{L^{1}(\widehat{d G}) \rightarrow L^{\infty}(d G)} \leq C_{\varepsilon}|h t|^{-n / 2}, \\
\left\|e^{-\gamma_{n} r} A_{\mathrm{pr}}(h) \widehat{O P}_{\iota}\left(a_{l, \text { inter }}^{ \pm}\right) e^{-i t h P} \widehat{O P}_{\iota}\left(a_{l, \text { inter }}^{ \pm}\right)^{*} A_{\mathrm{pr}}(h)^{*} e^{-\gamma_{n} r}\right\|_{L^{1}(\widehat{d G}) \rightarrow L^{\infty}(d G)} \leq C_{\varepsilon, \delta}|h t|^{-n / 2},
\end{aligned}
$$

for

$$
h \in(0,1] \text { and } 0 \leq \pm t \leq 2 h^{-1} .
$$

Recall that the important point in this lemma is (2-75), i.e., that it is sufficient to consider $t \geq 0$ for outgoing localizations, and $t \leq 0$ for incoming ones.

Proof. Define

$$
T_{\mathrm{s}}^{ \pm}(t, h, \varepsilon)=e^{-\gamma_{n} r} A_{\mathrm{pr}}(h) \widehat{O p}_{\iota}\left(a_{\mathrm{s}}^{ \pm}\right) e^{-i t P}, \quad T_{l, \text { inter }}^{ \pm}(t, h, \varepsilon, \delta)=e^{-\gamma_{n} r} A_{\mathrm{pr}}(h) \widehat{O p}_{\iota}\left(a_{l, \text { inter }}\right) e^{-i t P} .
$$

By (2-26) and (2-65) (with $q=2$ ), we have,

$$
\left\|T_{\mathrm{s}}^{ \pm}(t, h, \varepsilon)\right\|_{L^{2}(\widehat{d G}) \rightarrow L^{2}(d G)}+\left\|T_{l, \text { inter }}^{ \pm}(t, h, \varepsilon, \delta)\right\|_{L^{2}(\widehat{d G}) \rightarrow L^{2}(d G)} \leq C_{\varepsilon, \delta} \quad \text { for } h \in(0,1], t \in \mathbb{R} ;
$$

hence by the Keel-Tao Theorem [1998], the inequality (2-70) would follow from the estimates

$$
\begin{aligned}
\left\|T_{\mathrm{s}}^{ \pm}(t, h, \varepsilon) T_{\mathrm{s}}^{ \pm}(s, h, \varepsilon)^{*}\right\|_{L^{1}(d G) \rightarrow L^{\infty}(d G)} & \leq C_{\varepsilon}|t-s|^{-n / 2}, \\
\left\|T_{l, \text { inter }}^{ \pm}(t, h, \varepsilon) T_{l, \text { inter }}^{ \pm}(s, h, \varepsilon)^{*}\right\|_{L^{1}(d G) \rightarrow L^{\infty}(d G)} & \leq C_{\varepsilon, \delta}|t-s|^{-n / 2},
\end{aligned}
$$

for $h \in(0,1]$ and $t, s \in[0,1]$. Using the time rescaling $t \mapsto h t$, the equality $L^{1}(d G)=e^{-2 \gamma_{n} r} L^{1}(\widehat{d G})$, and the fact that the adjoint of (2-9) is given by $e^{\gamma_{n} r}$, we see that (2-76) and (2-77) are respectively equivalent to (2-73) and (2-74), for $h \in(0,1]$ and $|t| \leq 2 h^{-1}$.

The reduction (2-75) to $\pm t \geq 0$ is obtained similarly to [Bouclet and Tzvetkov 2007, Lemma 4.3]. We only recall here that it is based on the simple observation that the operators $T(t) T(s)^{*}$ considered above are of the form $B e^{-i(t-s) P} B^{*}$, so $L^{\infty}$ bounds on their Schwartz kernel for $\pm(t-s) \geq 0$ give automatically bounds for $\pm(t-s) \leq 0$ by taking the adjoints.

As we shall see, there are basically two reasons for choosing $\varepsilon$ small enough. The next result is the first condition.

Proposition 2.20 (Time $h^{-1}$ Isozaki-Kiada parametrix). For all $\varepsilon>0$ small enough and all $a_{\mathrm{s}}^{ \pm}$in $\mathscr{S}_{\text {hyp }}\left(\Gamma_{l, \mathrm{~s}}^{ \pm}(\varepsilon)\right)$, we can write

$$
e^{-i t h P} \widehat{O p_{\iota}}\left(a_{\mathrm{s}}^{ \pm}\right)^{*}=E_{\mathrm{IK}}^{ \pm}(t, h)+h^{n} R_{\mathrm{IK}}^{ \pm}(t, h),
$$

with

$$
\begin{aligned}
\left\|e^{-\gamma_{n} r} E_{\mathrm{IK}}^{ \pm}(t, h) e^{-\gamma_{n} r}\right\|_{L^{1}(\widehat{d G}) \rightarrow L^{\infty}(d G)} & \lesssim|h t|^{-n / 2}, \\
\left\|R_{\mathrm{IK}}^{ \pm}(t, h)\right\|_{L^{2}(\widehat{d G}) \rightarrow L^{2}(\widehat{d G})} & \lesssim 1,
\end{aligned}
$$

for

$$
h \in(0,1], \quad 0 \leq \pm t \leq 2 h^{-1} .
$$


Proof. By (2-38), the result follows from Theorem 5.1 and by a stationary phase argument justified by Propositions 7.2, 7.3, 7.6, Lemma 7.9 and Propositions 7.11, 7.12.

Proposition 2.20 is mainly an application of the Isozaki-Kiada parametrix. It has the following consequence.

Proposition 2.21 (Time $h^{-1}$ strongly incoming/outgoing dispersion estimates). For all $\varepsilon>0$ sufficiently small, (2-73) holds for all $h, t$ satisfying (2-75).

Proof. We first replace $\widehat{O p}_{\iota}\left(a_{\mathrm{s}}^{ \pm}\right)$by $O p_{\iota, \mathrm{pr}}\left(a_{\mathrm{s}}^{ \pm}\right)$to the left of $e^{-i t h P}$ in (2-73). The remainder term, which is $\mathcal{O}\left(h^{\infty}\right)$ in $\mathscr{L}\left(L^{2}(\widehat{d G})\right)$ by $(2-40)$, produces a term of size $\mathcal{O}\left(h^{\infty}\right)$ in $\mathscr{L}\left(L^{1}(\widehat{d G}), L^{\infty}(d G)\right)$ using $(2-65)$ (with $q=\infty$ ) and (2-67). We then use Proposition 2.20: the remainder term satisfies

$$
\left\|e^{-\gamma_{n} r} A_{\mathrm{pr}}(h) O p_{l, \mathrm{pr}}\left(a_{\mathrm{s}}^{ \pm}\right) e^{-i t h P} h^{n} R_{\mathrm{IK}}^{ \pm}(t, h) A_{\mathrm{pr}}(h)^{*} e^{-\gamma_{n} r}\right\|_{L^{1}(\widehat{d}) \rightarrow L^{\infty}(d G)} \lesssim 1 \lesssim|h t|^{-d / 2},
$$

and the main term $E_{\mathrm{IK}}^{ \pm}(t, h)$ gives the expected contribution via the use of (2-66), (2-68), and (2-49) for $O p_{\iota, \mathrm{pr}}\left(a_{\mathrm{s}}^{ \pm}\right)$.

The second condition on $\varepsilon$ will come from Proposition 2.24. It uses Proposition 2.16 which depends on some fixed small time which will be given by the following result.

Proposition 2.22 (Time 1 geometric optics). There exists $t_{\mathrm{WKB}}>0$ such that, for all $\varepsilon>0$ small enough and all symbol $a^{ \pm} \in \mathscr{S}_{\text {hyp }}\left(\Gamma_{\iota}^{ \pm}\left(R(\varepsilon), V_{\iota}, I, \frac{1}{2}\right)\right)$, we can write

$$
e^{-i t h P} \widehat{O p}_{\iota}\left(a^{ \pm}\right)^{*}=E_{\mathrm{WKB}}^{ \pm}(t, h)+h^{n} R_{\mathrm{WKB}}^{ \pm}(t, h),
$$

with

for

$$
\begin{aligned}
\left\|e^{-\gamma_{n} r} E_{\mathrm{WKB}}^{ \pm}(t, h) e^{-\gamma_{n} r}\right\|_{L^{1}(\widehat{d G}) \rightarrow L^{\infty}(d G)} & \lesssim|h t|^{-n / 2}, \\
\left\|R_{\mathrm{WKB}}^{ \pm}(t, h)\right\|_{L^{2}(\widehat{d G}) \rightarrow L^{2}(\widehat{d G})} & \lesssim 1,
\end{aligned}
$$

$$
h \in(0,1], \quad 0 \leq \pm t \leq t_{\mathrm{WKB}} .
$$

Proof. This follows from the stationary phase theorem, using the parametrix given in Theorem 6.1 and Propositions 7.2, 7.3, 7.6, and 7.8.

The first consequence of this proposition is the following result on short-time dispersion estimates, whose proof is completely similar to that of Proposition 2.21.

Proposition 2.23 (Time 1 dispersion estimates in intermediate areas). For all $\varepsilon>0$, all $\delta>0$ and all $a_{l, \text { inter }}^{ \pm}$satisfying (2-72), the estimate (2-74) holds for all $h, t$ satisfying (2-81).

We can now give the second condition on $\varepsilon$, also giving the choice of $\delta$. The proof is given in on page 65 (Section 6B).

Proposition 2.24 (Negligibility of $1 \lesssim t \lesssim h^{-1}$ dispersion estimates in intermediate areas). If $\varepsilon$ is small enough, we can choose $\delta>0$ small enough such that, for all $1 \leq l \leq L-1$, all

$$
b_{l, \text { inter }}^{ \pm} \in \mathscr{S}_{\text {hyp }}\left(\Gamma_{\iota, \text { inter }}^{ \pm}(\varepsilon, \delta ; l)\right),
$$

and all $N \geq 0$, we have

$$
\left\|\widehat{O p}_{\iota}\left(b_{l, \text { inter }}^{ \pm}\right) e^{-i t h P} \widehat{O p}_{\iota}\left(b_{l, \text { inter }}^{ \pm}\right)^{*}\right\|_{L^{2}(\widehat{d G}) \rightarrow L^{2}(\widehat{d G})} \leq C_{l, N} h^{N},
$$


for

$$
h \in(0,1], \quad t_{\mathrm{WKB}} \leq \pm t \leq 2 h^{-1} .
$$

This is, at least intuitively, a consequence of Proposition 2.16 with $\underline{t}=t_{\mathrm{WKB}}$ and of the Egorov theorem which shows that $e^{-i t h P} \widehat{O P}_{\iota}\left(b_{l, \text { inter }}^{ \pm}\right)^{*}$ lives semiclassically in the region $\Phi_{\iota}^{t}\left(\operatorname{supp}\left(b_{l, \text { inter }}^{ \pm}\right)\right)$.

We summarize the reasoning above as follows.

Proof of Theorem 1.2. Using Proposition 2.21, we first choose $\varepsilon_{0}>0$ small enough that, for all $\varepsilon \in$ $\left(0, \varepsilon_{0}\right]$, (2-73) holds for $0 \leq \pm t \leq 2 h^{-1}$. By possibly decreasing $\varepsilon_{0}$, we then choose $t_{\mathrm{WKB}}$ according to Proposition 2.22, uniformly with respect to $\varepsilon \in\left(0, \varepsilon_{0}\right]$. Next, according to Proposition 2.24, we fix $\varepsilon \in\left(0, \varepsilon_{0}\right]$ and $\delta>0$ small enough that (2-82) holds for $t_{\mathrm{WKB}} \leq \pm t \leq 2 h^{-1}$. Using (2-65), (2-67) and Proposition 2.24 with $N=n$ and $b_{l, \text { inter }}^{ \pm}=a_{l, \text { inter }}^{ \pm}$defined by (2-71), we have

$$
\left\|e^{-\gamma_{n} r} A_{\mathrm{pr}}(h) \widehat{O P}_{\iota}\left(a_{l, \text { inter }}^{ \pm}\right) e^{-i t h P} \widehat{O p}_{\iota}\left(a_{l, \text { inter }}^{ \pm}\right)^{*} A_{\mathrm{pr}}(h)^{*} e^{-\gamma_{n} r}\right\|_{L^{1}(\widehat{d G}) \rightarrow L^{\infty}(d G)} \leq C_{\varepsilon, \delta} \lesssim|h t|^{-n / 2},
$$

for $t_{\mathrm{WKB}} \leq \pm t \leq 2 h^{-1}$. On the other hand, (2-74) holds for $0 \leq \pm t \leq t_{\mathrm{WKB}}$, using Proposition 2.22. Therefore (2-74) holds for $0 \leq \pm t \leq 2 h^{-1}$. By Proposition 2.19, this proves (2-70) for all $a_{\iota}$ satisfying (2-69). By Proposition 2.18, this implies (2-63) which, by Proposition 2.9, implies Theorem 1.2.

\section{Estimates on the geodesic flow near infinity}

In this section, we describe some properties of the Hamiltonian flow of functions of the form

$$
p(r, \theta, \rho, \eta)=\rho^{2}+w(r) q(r, \theta, \eta),
$$

on $T^{*} \mathbb{R}_{+}^{n}=\mathbb{R}_{r}^{+} \times \mathbb{R}_{\theta}^{n-1} \times \mathbb{R}_{\rho} \times \mathbb{R}_{\eta}^{n-1}$. Here $q$ is an homogeneous polynomial of degree 2 with respect to $\eta$ and $w$ a positive function. In Section $3 \mathrm{~B}$, we will assume that $w(r)=e^{-2 r}$ but we start with more general cases in Section 3A.

The motivation for the study of (3-1) comes naturally from the form of the principal symbol $p_{\iota}$ of $P$ given by (2-14).

We emphasize that the symbol $p$ considered in this section is defined on $T^{*} \mathbb{R}_{+}^{n}$ whereas $p_{\iota}$ is only defined on a subset of the form $T^{*}\left(R_{\mathscr{K}},+\infty\right) \times V_{\iota}$. The results of Section 3B will nevertheless hold for $p_{\iota}$ as well with no difficulty for we shall have a good localization of the flow in the regions we consider (see Corollary 3.10).

3A. A general result. Let $w=w(r)$ be a smooth function on $\mathbb{R}^{+}=(0,+\infty)$ such that

$$
w>0, \quad w^{\prime}<0, \quad\left(\frac{w^{\prime}}{w}\right)^{\prime} \geq 0
$$

and, for some $0<\gamma<1$,

$$
\limsup _{r \rightarrow+\infty} \int_{r}^{(1+\gamma) r} \frac{w^{\prime}}{w} \in[-\infty, 0) .
$$

Note that $\lim _{r \rightarrow+\infty} w(r)$ exists, by (3-2), and that (3-3) implies that this limit must be 0 . Note also that, for all $R>0$, we have

$$
w(r) \lesssim 1 \quad \text { and } \quad\left|w^{\prime}(r)\right| \lesssim w(r) \quad \text { for } r \in[R,+\infty)
$$


These assumptions are satisfied for instance by $w(r)=r^{-2}$ or $w(r)=e^{-2 r}$.

We assume that $q$ is an homogeneous polynomial of degree 2 with respect to $\eta$ of the form

$$
q(r, \theta, \eta)=q_{0}(\theta, \eta)+q_{1}(r, \theta, \eta)
$$

with $q_{0}, q_{1}$ homogeneous polynomials of degree 2 with respect to $\eta$ satisfying, for some $0<\tau \leq 1$,

$$
\begin{aligned}
\left|\partial_{\theta}^{\alpha} \partial_{\eta}^{\beta} q_{0}(\theta, \eta)\right| & \lesssim\langle\eta\rangle^{2-|\beta|}, \\
\left|\partial_{r}^{j} \partial_{\theta}^{\alpha} \partial_{\eta}^{\beta} q_{1}(r, \theta, \eta)\right| & \lesssim\langle r\rangle^{-\tau-j}\langle\eta\rangle^{2-|\beta|},
\end{aligned}
$$

and, for some $C>0$,

$$
C^{-1}|\eta|^{2} \leq q(r, \theta, \eta) \leq C|\eta|^{2},
$$

for $(r, \theta, \eta) \in \mathbb{R}^{+} \times \mathbb{R}^{n-1} \times \mathbb{R}^{n-1}$. The latter implies, by possibly increasing $C$, that

$$
C^{-1}|\eta|^{2} \leq q_{0}(\theta, \eta) \leq C|\eta|^{2}, \quad(\theta, \eta) \in \mathbb{R}^{n-1} \times \mathbb{R}^{n-1} .
$$

Setting $q^{\prime}=\partial_{r} q\left(=\partial_{r} q_{1}\right)$, we finally assume that,

$$
\frac{q^{\prime}}{q} \times \frac{w}{w^{\prime}} \rightarrow 0 \quad \text { as } \quad r \rightarrow+\infty
$$

uniformly with respect to $\theta \in \mathbb{R}^{n-1}$ and $\eta \in \mathbb{R}^{n-1} \backslash 0$.

The Hamiltonian flow $\Phi^{t}=\left(r^{t}, \theta^{t}, \rho^{t}, \eta^{t}\right)$, generated by $p$, is the solution to the system

$$
\left\{\begin{array}{l}
\dot{r}=2 \rho \\
\dot{\theta}=w \partial q / \partial \eta \\
\dot{\rho}=-w^{\prime} q-w q^{\prime}, \\
\dot{\eta}=-w \partial q / \partial \theta
\end{array}\right.
$$

with initial condition

$$
\left(r^{t}, \theta^{t}, \rho^{t}, \eta^{t}\right)_{\mid t=0}=(r, \theta, \rho, \eta) .
$$

Our main purpose is to show that, if $\rho>-p^{1 / 2}$ (with $p=p(r, \theta, \rho, \eta)$ ) and $r$ is large enough, then $\Phi^{t}$ is defined for all $t \geq 0$ and $r_{t} \rightarrow+\infty$ as $t \rightarrow+\infty$ (we will obtain a similar result for $t \leq 0$ provided $\rho<p^{1 / 2}$ ). This result relies mainly on the following remark: if $\eta \neq 0$, we can write

$$
-w^{\prime} q-w q^{\prime}=-\frac{w^{\prime}}{w}\left(p-\rho^{2}\right)\left(1+\frac{w}{w^{\prime}} \times \frac{q^{\prime}}{q}\right) .
$$

Using (3-9) and the negativity of $w^{\prime} / w$, this shows that, for all $\varepsilon>0$, we can find $R>0$ such that

$$
-w^{\prime} q-w q^{\prime} \geq-(1-\varepsilon)\left(p-\rho^{2}\right) \frac{w^{\prime}}{w}, \quad \text { on }[R,+\infty)_{r} \times \mathbb{R}_{\theta}^{n-1} \times \mathbb{R}_{\rho} \times \mathbb{R}_{\eta}^{n-1}
$$

which we shall exploit to prove that $\dot{\rho} \geq 0$.

In the following lemma and in the sequel, we shall use extensively the shorter notation

$$
p=p(r, \theta, \rho, \eta)
$$


Lemma 3.1. Denote by $\left(-t_{-}, t_{+}\right)\left(t_{ \pm} \in(0,+\infty]\right)$ the maximal interval on which the solution of $(3-10)$, with initial condition (3-11), is defined. Then

$$
t_{ \pm} \geq \frac{r}{2 p^{1 / 2}}
$$

Furthermore, either $r_{t} \rightarrow 0$ as $t \rightarrow t_{+}\left(\right.$resp. $\left.t \rightarrow-t_{-}\right)$or $t_{+}=+\infty\left(\right.$ resp. $\left.t_{-}=+\infty\right)$.

Note that, if $p(r, \theta, \rho, \eta)=0$, i.e., $\rho=0$ and $\eta=0$, then it is trivial that $t_{ \pm}=+\infty$.

Proof. We will only consider the case of $t_{+}$, the one of $t_{-}$being similar. By the conservation of energy we have $\left|\rho^{t}\right| \leq p^{1 / 2}$ thus, for $t \in\left[0, t_{+}\right)$, $\dot{r}^{t}$ is bounded,

$$
\left|r^{t}-r\right| \leq 2 t p^{1 / 2}
$$

and $r^{t} \geq r-2 t p^{1 / 2}$. We now argue by contradiction and assume that $t_{+}<r / 2 p^{1 / 2}$ (in particular, that $t_{+}$is finite). Then $r_{+}:=r-2 t_{+} p^{1 / 2}>0$ and $r_{t} \geq r_{+}$for all $t \in\left[0, t_{+}\right)$. Furthermore, by (3-7), we have $\left|w \partial_{\eta} q\right| \leq C(w q+w) \leq C(p+w)$, with $w$ bounded on $\left[r_{+},+\infty\right)$, hence $\dot{\theta}^{t}$ is bounded on $\left[0, t_{+}\right)$. One shows similarly that $\dot{\rho}^{t}$ and $\dot{\eta}^{t}$ are bounded on $\left[0, t_{+}\right)$, using that $\left|w^{\prime}\right| \lesssim w$ on $\left[r_{+},+\infty\right)$ for $\dot{\rho}$. This implies that $\lim _{t \rightarrow t_{+}}\left(r^{t}, \theta^{t}, \rho^{t}, \eta^{t}\right)$ exists and belongs to $(0,+\infty) \times \mathbb{R}^{n-1} \times \mathbb{R} \times \mathbb{R}^{n-1}$. The solution can therefore be continued beyond $t_{+}$, which yields the contradiction.

We now consider the second statement. Assume that $t_{+}<+\infty$. We must show that $r^{t} \rightarrow 0$ as $t \rightarrow t_{+}$. Assume that this is wrong. Then there exists $R>0$ small enough and a sequence $t_{k} \rightarrow t_{+}$such that $r^{t_{k}} \geq R$ for all $k \geq 0$. On the other hand, by energy conservation, we have $\left|r^{t}-r^{s}\right| \leq 2 p^{1 / 2}|t-s|$ for all $t, s \in\left[0, t_{+}\right)$, hence

$$
r^{t} \geq r^{t_{k}}-2 p^{1 / 2}\left|t-t_{k}\right| \geq R / 2
$$

provided $\left|t-t_{k}\right| \leq R / 4 p^{1 / 2}$. Since $t_{k}$ can be chosen as close to $t_{+}$as we want, there exists $\varepsilon>$ 0 small enough such that $r^{t} \geq R / 2$ for $t \in\left[t_{+}-\varepsilon, t_{+}\right)$. Then, by the same argument as above, $\lim _{t \rightarrow t_{+}}\left(r^{t}, \theta^{t}, \rho^{t}, \eta^{t}\right)$ exists and belongs to $(0,+\infty) \times \mathbb{R}^{n-1} \times \mathbb{R} \times \mathbb{R}^{n-1}$. The solution can be continued beyond $t_{+}$; hence $t_{+}=+\infty$, which is a contradiction.

Lemma 3.2. Let $0<\varepsilon<1$. For any $R>0$ such that (3-12) holds, we have:

(i) If $r^{t_{0}} \geq R$ and $\rho^{t_{0}}>0$ for some $t_{0} \in\left[0, t_{+}\right)$, then $t_{+}=+\infty$ and

$$
r^{t} \geq R, \quad \rho^{t} \geq \rho^{t_{0}}, \quad r^{t} \geq r^{t_{0}}+2\left(t-t_{0}\right) \rho^{t_{0}} \quad \text { for all } t \geq t_{0} .
$$

(ii) If $r^{t_{0}} \geq R$ and $\rho^{t_{0}}<0$ for some $t_{0} \in\left(-t_{-}, 0\right]$, then $-t_{-}=-\infty$ and

$$
r^{t} \geq R, \quad \rho^{t} \leq \rho^{t_{0}}, \quad r^{t} \geq r^{t_{0}}+2\left(t-t_{0}\right) \rho^{t_{0}} \quad \text { for all } t \leq t_{0} .
$$

Proof. As in Lemma 3.1, we only consider the case of $t_{+}$. It suffices to show that

$$
r^{t} \geq R \text { for all } t \in\left[t_{0}, t_{+}\right) .
$$

Indeed, if this is true, Lemma 3.1 shows that $t_{+}=+\infty$ and then, by (3-12), we have $\dot{\rho}^{t} \geq 0$, whence $\rho^{t} \geq \rho^{t_{0}}$ and $r^{t}-r^{t_{0}} \geq 2 \rho^{t_{0}}\left(t-t_{0}\right)$. Let us prove (3-14). Consider the set

$$
I=\left\{t \in\left[t_{0}, t_{+}\right): r^{s} \geq R \text { and } \rho^{s} \geq \rho^{t_{0}} \text { for all } s \in\left[t_{0}, t\right]\right\} .
$$


It is clearly an interval containing $t_{0}$ and we set $T:=\sup I$. By continuity, $\rho^{t} \geq \rho^{t_{0}} / 2>0$ for $t$ in a small neighborhood $J$ of $t_{0}$. This implies that $\dot{r}^{t}>0$ on $J$, hence that $r^{t} \geq r^{t_{0}} \geq R$ on $J \cap\left[t_{0}, t_{+}\right)$and thus that $\dot{\rho}^{t} \geq 0$ on $J \cap\left[t_{0}, t_{+}\right)$which in turn shows that $\rho^{t} \geq \rho^{t_{0}}$ on $J \cap\left[t_{0}, t_{+}\right)$. This proves that $T>t_{0}$. Then, on $\left[t_{0}, T\right)$, we have

$$
r^{t} \geq R, \quad \rho^{t} \geq \rho^{t_{0}} .
$$

Now assume, by contradiction, that $T<t_{+}$. Then (3-15) holds on $\left[t_{0}, T\right]$ and in particular we have $r^{T} \geq r^{t_{0}}+2\left(T-t_{0}\right) \rho^{t_{0}}>r^{t_{0}}$. Thus $r^{t} \geq R$ in a neighborhood of $T$ and this implies that $\dot{\rho}^{t} \geq 0$ in this neighborhood. Hence there exists $T^{\prime}>T$ such that (3-15) holds on $\left[t_{0}, T^{\prime}\right]$ yielding a contradiction.

To state the next result, we define $l \in(0,+\infty]$ as

$$
l=-\limsup _{r \rightarrow+\infty} \int_{r}^{(1+\gamma) r} \frac{w^{\prime}}{w}
$$

and we choose an arbitrary $\sigma \in \mathbb{R}$ such that

$$
0<\sigma<\left\{\begin{array}{cl}
-\frac{2}{l}+\left(\frac{4}{l^{2}}+1\right)^{1 / 2} & \text { if } l<+\infty \\
1 & \text { if } l=+\infty
\end{array}\right.
$$

Note that $0<-\frac{2}{l}+\left(\frac{4}{l^{2}}+1\right)^{1 / 2}<1$ if $l$ is finite, and that (3-17) is equivalent to

$$
\left(1-\sigma^{2}\right) l / 2>2 \sigma>0 .
$$

Proposition 3.3. For any $\sigma$ satisfying (3-17), there exists $R_{w, \gamma, \sigma}>0$ large enough that the following property holds. Let $r>R_{w, \gamma, \sigma}$. Then:

(i) If $\rho>-\sigma p^{1 / 2}$, then $t_{+}=+\infty$ and

$$
r^{t} \geq \max \left((1-\gamma) r,(1-\gamma-\sigma \gamma) r+2 \sigma p^{1 / 2}|t|\right)
$$

for all $t \geq 0$.

(ii) If $\rho<\sigma p^{1 / 2}$, then $-t_{-}=-\infty$ and (3-18) holds for $t \leq 0$.

This proposition means that, by choosing initial data with $r$ large enough and $\rho>-\sigma p^{1 / 2}$ (resp. $\rho<\sigma p^{1 / 2}$ ), the forward (resp. backward) trajectory lies in a neighborhood of infinity. In particular, the forward (resp. backward) flow starting at $(r, \theta, \rho, \eta)$, with $\rho>-\sigma p^{1 / 2}$ (resp. $\left.\rho<\sigma p^{1 / 2}\right)$ depends only on the values of $p$ on $[(1-\gamma) r,+\infty) \times \mathbb{R}^{n-1} \times \mathbb{R} \times \mathbb{R}^{n-1}$.

Proof. We only consider the case where $\rho>-\sigma p^{1 / 2}$, the case where $\rho<\sigma p^{1 / 2}$ being similar. If $l<\infty$, (3-17) allows one to choose $0<\varepsilon<1$ such that

$$
(1-\varepsilon)^{2}\left(1-\sigma^{2}\right) l / 2 \geq 2 \sigma .
$$

If $l=\infty$, we choose an arbitrary $\varepsilon \in(0,1)$. We next choose $R$ so that (3-12) holds with the above choice of $\varepsilon$. If $\rho \geq \sigma p^{1 / 2}$ (recall that $p^{1 / 2}>0$ since $\rho>-\sigma p^{1 / 2}$ ) and $r \geq R$, then Lemma 3.2 shows that the result holds with $R_{w, \gamma, \sigma}=R$. We can therefore assume that $\rho<\sigma p^{1 / 2}$. Set

$$
R_{1}=(1-\gamma)^{-1} R, \quad T=\gamma r / 2 p^{1 / 2} .
$$


By Lemma 3.1, we have $t_{+}>T$ and, if $r \geq R_{1}$,

$$
r^{t} \geq r-2 t p^{1 / 2} \geq(1-\gamma) r \geq R \text { for } t \in[0, T]
$$

Using (3-12), this implies that $\dot{\rho}^{t} \geq 0$ on $[0, T]$ and hence that $\rho^{t} \geq-\sigma p^{1 / 2}$ for all $t \in[0, T]$. We now prove by contradiction that there exists $t \in[0, T]$ such that $\rho^{t} \geq \sigma p^{1 / 2}$. If this is wrong, we have $\left(\rho^{t}\right)^{2} \leq \sigma^{2} p$ on $[0, T]$, thus (3-12) shows that, for all $t \in[0, T]$,

$$
\dot{\rho}^{t} \geq-(1-\varepsilon)\left(1-\sigma^{2}\right) p \frac{w^{\prime}}{w}\left(r^{t}\right) \geq-(1-\varepsilon)\left(1-\sigma^{2}\right) p \frac{w^{\prime}}{w}\left(r+2 t p^{1 / 2}\right),
$$

using the third estimate of (3-2) and the fact that $r^{t} \leq r+2 t p^{1 / 2}$ in the second inequality. By integration over $[0, T]$, we get

$$
\rho^{T}-\rho \geq-(1-\varepsilon)\left(1-\sigma^{2}\right) p^{1 / 2} \frac{1}{2} \int_{r}^{(1+\gamma) r} \frac{w^{\prime}}{w},
$$

using the second equality in (3-20). Fix $R_{2}$ such that, for all $r>R_{2}$,

$$
-\int_{r}^{(1+\gamma) r} \frac{w^{\prime}}{w}>\left\{\begin{array}{cl}
(1-\varepsilon) l & \text { if } l<+\infty \\
\frac{4 \sigma}{(1-\varepsilon)\left(1-\sigma^{2}\right)} & \text { if } l=+\infty
\end{array}\right.
$$

With such a choice (and (3-19) if $l$ is finite), we see that, if $r \geq \max \left(R_{1}, R_{2}\right)$, (3-21) implies that $\rho^{T}-\rho \geq 2 \sigma p^{1 / 2}$ and hence that $\rho^{T} \geq \sigma p^{1 / 2}$ which yields the expected contradiction.

In summary, we have shown that for any $r \geq \max \left(R_{1}, R_{2}\right)$ and any $\rho>-\sigma p^{1 / 2}$, there exists $t_{0} \in[0, T]$ such that $\rho^{t_{0}} \geq \sigma p^{1 / 2}>0$ and $r^{t_{0}} \geq R$, hence $t_{+}=+\infty$ by Lemma 3.2. Furthermore, $r^{t} \geq(1-\gamma) r$ on $[0, T]$ and $r^{t} \geq r^{T}+2(t-T) \sigma p^{1 / 2} \geq(1-(1+\sigma) \gamma) r+2 t \sigma p^{1 / 2}$ on $[T,+\infty)$. The result follows since

$$
\max \left((1-\gamma) r,(1-\gamma-\sigma \gamma) r+2 \sigma p^{1 / 2} t\right)= \begin{cases}(1-\gamma) r & \text { if } t \in[0, T] \\ (1-\gamma-\sigma \gamma) r+2 \sigma p^{1 / 2} t & \text { if } t>T .\end{cases}
$$

3B. The asymptotically hyperbolic case. We will now prove more precise estimates on the Hamiltonian flow of $p$ when

$$
w(r)=e^{-2 r} .
$$

In that case, the conditions (3-2), (3-3) and (3-9) are fulfilled, with any $0<\gamma<1$ in (3-3) and we have $l=+\infty$ in $(3-16)$.

We shall need the following improvement of Proposition 3.3.

Proposition 3.4. Let $0<\sigma<1$. There exist $R_{\sigma}>0$ and $C_{\sigma}>0$ such that: if $r \geq R_{\sigma}$ and $\rho>-\sigma p^{1 / 2}$ (resp. $\rho<\sigma p^{1 / 2}$ ), then

$$
\left.r^{t} \geq r+2 \sigma p^{1 / 2}|t|-C_{\sigma}, \quad \text { for all } t \geq 0 \text { (resp. } t \leq 0\right) .
$$

The improvement consists in replacing $(1-\gamma-\sigma \gamma) r$ in the estimate (3-18) by $r-C_{\sigma}$.

Proof. Here again we only consider the case $t \geq 0$. By Proposition 3.3, we may assume that $r^{t} \geq R$ for all $t \geq 0$, with $R$ large enough so that (3-12) holds with $\varepsilon=\frac{1}{2}$. This implies that

$$
\dot{\rho}^{t}=2 e^{-2 r^{t}} q\left(r^{t}, \theta^{t}, \eta^{t}\right)-e^{-2 r^{t}} \partial_{r} q_{1}\left(r^{t}, \theta^{t}, \eta^{t}\right) \geq e^{-2 r^{t}} q\left(r^{t}, \theta^{t}, \eta^{t}\right)=p-\left(\rho^{t}\right)^{2} .
$$


If $\rho \geq \sigma p^{1 / 2}$, then the result follows from Lemma 3.2 (with $C_{\sigma}=0$ ). If $\rho<\sigma p^{1 / 2}$, we will show that, with $T=2 \sigma p^{-1 / 2} /\left(1-\sigma^{2}\right)$, there exists $t \in[0, T]$ such that $\rho^{t} \geq \sigma p^{1 / 2}$. Assume that this is wrong. Then $\left(\rho^{t}\right)^{2} \leq \sigma^{2} p$ on $[0, T]$ and by integrating the above estimate on $\dot{\rho}^{t}$, we get

$$
\rho^{T}-\rho \geq T\left(1-\sigma^{2}\right) p=2 \sigma p^{1 / 2} .
$$

This proves that $\rho^{T} \geq \sigma p^{1 / 2}$ which is a contradiction. Therefore, by Lemma 3.2, we see that $r^{t}-r^{T} \geq$ $2 \sigma p^{1 / 2}(t-T)$ for $t \geq T$. On the other hand, we have $r^{t} \geq r-2 p^{1 / 2} t$ for $t \in[0, T]$. The latter implies that $r^{t} \geq r+2 \sigma p^{1 / 2} t-2 p^{1 / 2}(1+\sigma) t \geq r+2 \sigma p^{1 / 2} t-4 \sigma /(1-\sigma)$ for $t \in[0, T]$. This holds in particular for $t=T$ and then for $t \geq T$. Thus the results holds with $C_{\sigma}=4 \sigma /(1-\sigma)$.

We have so far only studied some localization properties of $\Phi^{t}$, the Hamiltonian flow of $p$. We shall now give estimates on derivatives of $\Phi^{t}$. We start with the following lemma giving some rough estimates. They will serve as a priori estimates for the proof of Proposition 3.8 below.

Lemma 3.5. For all $0<\sigma<1$, there exists $R>0$ such that, for all $(r, \theta, \rho, \eta) \in T^{*} \mathbb{R}_{+}^{n}$ satisfying

$$
r>R, \quad \pm \rho>-\sigma p^{1 / 2}, \quad p \in\left(\frac{1}{4}, 4\right),
$$

and all $\pm t \geq 0$, we have

$$
\left|e^{r|\beta|} \partial_{\eta}^{\beta} \partial_{r}^{j} \partial_{\theta}^{\alpha} \partial_{\rho}^{k}\left(\Phi^{t}-\Phi^{0}\right)(r, \theta, \rho, \eta)\right| \lesssim\langle t\rangle .
$$

Note the $e^{r|\beta|}$ factor in front of the derivatives.

We will need two lemmas. The first one, proved by induction, is a soft version of the classical Faà di Bruno formula.

Lemma 3.6. Let $\Omega_{1} \subset \mathbb{R}^{n_{1}}, \Omega_{2} \subset \mathbb{R}^{n_{2}}$ be open subsets, with $n_{1}, n_{2} \geq 1$. Consider smooth maps $y=$ $\left(y_{1}, \ldots, y_{n_{2}}\right): \Omega_{1} \rightarrow \Omega_{2}$ and $Z: \Omega_{1} \times \Omega_{2} \rightarrow \mathbb{R}^{n_{3}}$, with $n_{3} \geq 1$. Then, for all $|\gamma| \geq 1$,

$$
\partial_{x}^{\gamma}(Z(x, y(x)))=\left(\partial_{y} Z\right)(x, y(x)) \partial_{x}^{\gamma} y(x)+\left(\partial_{x}^{\gamma} Z\right)(x, y(x))+R_{\gamma}(x)
$$

where $R_{\gamma}(x)$ vanishes identically if $|\gamma|=1$ and, otherwise, is a linear combination of

$$
\left(\partial_{x}^{\gamma-\gamma^{\prime}} \partial_{y}^{v} Z\right)(x, y(x))\left(\partial_{x}^{\gamma_{1}^{1}} y_{1}(x) \ldots \partial_{x}^{\gamma_{v_{1}}^{1}} y_{1}(x)\right) \ldots\left(\partial_{x}^{\gamma_{1}^{n_{2}}} y_{n_{2}}(x) \ldots \partial_{x}^{\gamma_{v_{n_{2}}}^{n_{2}}} y_{n_{2}}(x)\right),
$$

with $\gamma, \gamma^{\prime}, \gamma_{j}^{k} \in \mathbb{N}_{0}^{n_{1}}, v=\left(v_{1}, \ldots, v_{n_{2}}\right) \in \mathbb{N}_{0}^{n_{2}}$ satisfying $\gamma^{\prime} \neq 0, v \neq 0$ and

$$
\gamma^{\prime} \leq \gamma, \quad 2 \leq|v|+\left|\gamma-\gamma^{\prime}\right| \leq|\gamma|, \quad \gamma_{1}^{1}+\cdots+\gamma_{\nu_{1}}^{1}+\cdots+\gamma_{1}^{n_{2}}+\cdots+\gamma_{v_{n_{2}}}^{n_{2}}=\gamma^{\prime},
$$

and using the convention that $\partial_{x}^{\gamma_{1}^{k}} y_{k}(x) \ldots \partial_{x}^{\gamma_{v_{k}}^{k}} y_{k}(x) \equiv 1$ if $v_{k}=0$ (if $v_{k} \neq 0$ then $\gamma_{1}^{k}, \ldots, \gamma_{v_{k}}^{k}$ are all nonzero).

In the second lemma, we consider the linear differential equation

$$
\dot{X}=A(t) X+Y(t),
$$

where $A(\cdot)$ is a continuous map from $[0,+\infty)$ to the space $\left.\mu_{N \times N}(\mathbb{R})\right)$ of $N \times N$ matrices with real entries, for some $N \geq 1$, and $Y(\cdot) \in C\left([0,+\infty), \mathbb{C}^{N}\right)$. We assume that $A(\cdot)$ belongs to a subset $\mathscr{B} \subset C\left([0,+\infty), \mu_{N \times N}(\mathbb{R})\right)$ for which there exist $\delta_{\mathscr{B}}>0$ and $C_{\mathscr{B}}>0$ such that

$$
\|A(t)\| \leq C_{\mathscr{B}} e^{-\delta_{\mathscr{B}} t} \quad \text { for all } t \geq 0 \text { and } A(\cdot) \in \mathscr{B},
$$


with $\|\cdot\| \|$ a matrix norm associated to the norm $\|\cdot\|$ on $\mathbb{C}^{N}$, i.e., such that $\|M Z\| \leq\|M\|\|Z\|$, for all $M \in M_{N \times N}(\mathbb{R})$ and $Z \in \mathbb{C}^{N}$.

Lemma 3.7. There exists $C>0$ such that, for all $A(\cdot) \in \mathscr{B}$ and all $Y(\cdot)$ satisfying

$$
\int_{0}^{\infty}\|Y(t)\| d t<\infty
$$

the solutions $X(\cdot)$ of (3-24) satisfy

$$
\|X(t)\| \leq C\left(\|X(0)\|+\int_{0}^{\infty}\|Y(s)\| d s\right) \quad \text { for } t \geq 0 .
$$

Proof. First fix $0<\delta<\delta_{\mathscr{B}}$ and $\varepsilon=\delta_{\mathscr{B}}-\delta$. Choose $T>0$ such that $C_{\mathscr{乃}} e^{-\delta_{\mathscr{B}} t} \leq \varepsilon$ for $t \geq T$. By Gronwall's lemma, we have

$$
\begin{array}{ll}
\|X(t)\| \leq\left(\|X(T)\|+\int_{T}^{\infty}\|Y(s)\| d s\right) e^{\varepsilon(t-T)} & \text { for } t \geq T, \\
\|X(t)\| \leq\left(\|X(0)\|+\int_{0}^{T}\|Y(s)\| d s\right) e^{C_{\mathscr{乃}} T} & \text { for } t \in[0, T] .
\end{array}
$$

These two inequalities give, for some $C$ depending only on $C_{\mathscr{B}}, \delta_{\mathscr{B}}, \delta$ and $T$,

$$
\|X(t)\| \leq C\left(\|X(0)\|+\int_{0}^{\infty}\|Y(s)\| d s\right) e^{\varepsilon t} \quad \text { for } t \geq 0 .
$$

Used as an a priori estimate in (3-24), this yields

$$
\|\dot{X}(t)\| \leq\|Y(t)\|+C C_{\Re} e^{-\delta t}\left(\|X(0)\|+\int_{0}^{\infty}\|Y(s)\| d s\right) \quad \text { for } t \geq 0,
$$

which implies (3-25).

Proof of Lemma 3.5. As before, we only prove the result for $t \geq 0$. For $|\beta|+j+|\alpha|+k=0$, the result is a consequence of the motion equations (3-10) and energy conservation. Indeed, for $r^{t}-r$, the estimate follows directly from (3-13). Next, the equation of motion for $\theta$, together with (3-7) and Proposition 3.4, shows that

$$
\left|\dot{\theta}^{t}\right| \lesssim e^{-2 r^{t}}\left|\eta^{t}\right| \lesssim e^{-2 r^{t}}\left\langle\eta^{t}\right\rangle^{2} \lesssim 1+p
$$

hence that $\left|\theta^{t}-\theta\right| \lesssim\langle t\rangle$ by integration. One similarly shows that $\left|\rho^{t}-\rho\right|+\left|\eta^{t}-\rho\right| \lesssim\langle t\rangle$. We now consider the derivatives and write, for simplicity, $\partial^{\gamma}=\partial_{\eta}^{\beta} \partial_{r}^{j} \partial_{\theta}^{\alpha} \partial_{\rho}^{k}$. Denoting by $H_{p}$ is the Hamiltonian vector field of $p$ and applying $\partial^{\gamma}$ to (3-10), we obtain

$$
e^{r|\beta|} \partial^{\gamma} \dot{\Phi}^{t}=\left(d H_{p}\right)\left(\Phi^{t}\right) e^{r|\beta|} \partial^{\gamma} \Phi^{t}+R(t)
$$

where, by Lemma 3.6, $R(t)$ vanishes if $|\gamma|=1$ or, if $|\gamma| \geq 2$, is a linear combination of

$$
\left(\partial^{v} H_{p}\right)\left(\Phi^{t}\right) e^{r|\beta|}\left(\partial^{\gamma_{1}^{1}} r^{t} \cdots \partial^{\gamma_{\nu_{1}}^{1}} r^{t}\right) \cdots\left(\partial^{\gamma_{1}^{2 n}} \eta_{n-1}^{t} \cdots \partial^{\gamma_{v_{2 n}}^{2 n}} \eta_{n-1}^{t}\right) .
$$


Here $v=\left(v_{1}, \ldots, v_{2 n}\right)$ is of length at least 2 , all the derivatives of $\Phi^{t}$ involved in $R(t)$ are of strictly smaller order than $\gamma$ (meaning that $\gamma_{l_{i}}^{i} \leq \gamma$ and $\gamma_{l_{i}}^{i} \neq \gamma$ ), and

$$
2 \leq|v| \leq|\gamma|, \quad \gamma_{1}^{1}+\cdots+\gamma_{v_{2 n}}^{2 n}=\gamma .
$$

Writing $d H_{p}$ as a matrix, we have

$$
d H_{p}=\left(\begin{array}{cccc}
0 & 0 & 2 & 0 \\
0 & 0 & 0 & 0 \\
0 & 0 & 0 & 0 \\
0 & 0 & 0 & 0
\end{array}\right)+e^{-2 r}\left(\begin{array}{cccc}
0 & 0 & 0 & 0 \\
\partial_{r \eta}^{2} q_{1}-2 \partial_{\eta} q & \partial_{\theta \eta}^{2} q & 0 & \partial_{\eta \eta}^{2} q \\
4 \partial_{r} q_{1}-4 q-\partial_{r r}^{2} q_{1} & 2 \partial_{\theta} q-\partial_{\theta r}^{2} q_{1} & 0 & 2 \partial_{\eta} q-\partial_{\eta r}^{2} q_{1} \\
2 \partial_{\theta} q-\partial_{r \theta}^{2} q_{1} & -\partial_{\theta \theta}^{2} q & 0 & -\partial_{\eta \theta}^{2} q
\end{array}\right) .
$$

Defining $M$ as the first (constant) matrix of the right-hand side and using Proposition 3.4, we have

$$
\left|d H_{p}\left(\Phi^{t}\right)-M\right| \lesssim e^{-2 r^{t}}\left\langle\eta^{t}\right\rangle^{2} \lesssim e^{-2 r-2 \sigma t}\left(\langle\eta\rangle^{2}+\langle t\rangle^{2}\right) \lesssim e^{-\sigma t},
$$

using that $2 p^{1 / 2} \geq 1$ and that $e^{-2 r}\langle\eta\rangle^{2}$ is bounded, by (3-23). We then set

$$
\begin{aligned}
& A(t)=e^{-t M}\left(d H_{p}\left(\Phi^{t}\right)-M\right) e^{t M}, \\
& X(t)=e^{-t M} e^{r|\beta|} \partial^{\gamma} \Phi^{t}-e^{r|\beta|} \partial^{\gamma} \Phi^{0}, \\
& Y(t)=e^{-t M} R(t)+A(t) e^{r|\beta|} \partial^{\gamma} \Phi^{0},
\end{aligned}
$$

so that

$$
\dot{X}(t)=A(t) X(t)+Y(t), \quad X(0)=0 .
$$

Noting that $M^{2}=0$, we have

$$
\exp ( \pm t M)=1 \pm t M, \quad|\exp ( \pm t M)| \lesssim\langle t\rangle
$$

thus

$$
|A(t)| \lesssim e^{-\sigma t}\langle t\rangle^{2} \lesssim e^{-\sigma t / 2}
$$

To estimate $X(t)$ by Lemma 3.7, we still need to estimate $Y(t)$. We first assume that $\partial^{\gamma}=\partial_{\eta}^{\beta}$ with $|\beta|=1$. We then have $R(t)=0$ and

$$
A(t) e^{r|\beta|} \partial^{\gamma} \Phi^{0}=e^{-t M}\left(\partial_{\eta}^{\beta} H_{p}\right)\left(\Phi^{t}\right) e^{r},
$$

since $M \partial_{\eta}^{\beta} \Phi^{0}=0$. By Proposition 3.4 and (3-23) again, we obtain

$$
\left|\left(\partial_{\eta}^{\beta} H_{p}\right)\left(\Phi^{t}\right)\right| \lesssim e^{-2 r-2 \sigma t}\left\langle\eta^{t}\right\rangle \lesssim e^{-r-\sigma t},
$$

so that $|Y(t)| \lesssim e^{-\sigma t / 2}$. Using (3-29) and Lemma 3.7, we get $|X(t)| \lesssim 1$. Since $M \partial_{\eta}^{\beta} \Phi^{0}=0$, we can rewrite $X(t)=e^{-t M} e^{r} \partial_{\eta}^{\beta}\left(\Phi^{t}-\Phi^{0}\right)$ and, using (3-28), finally get

$$
\left|e^{r} \partial_{\eta}^{\beta}\left(\Phi^{t}-\Phi^{0}\right)\right| \lesssim\langle t\rangle
$$

The other first-order derivatives of $\Phi^{t}-\Phi^{0}$ are studied similarly (note that there is no $e^{r}$ factor then), by showing that $X(t)$ is bounded and using that $X(t)=e^{-t M} \partial^{\gamma}\left(\Phi^{t}-\Phi^{0}\right)+\left(e^{-t M}-1\right) \partial^{\gamma} \Phi^{0}$ with (3-28) to get

$$
\left|\partial^{\gamma}\left(\Phi^{t}-\Phi^{0}\right)\right| \lesssim\langle t\rangle
$$


For higher-order derivatives, $\partial^{\gamma} \Phi^{0}=0$ and $\partial^{\gamma}\left(\Phi^{t}-\Phi^{0}\right)=\partial^{\gamma} \Phi^{t}$. Furthermore, since the derivatives of $\Phi^{t}$ involved in $R(t)$ are of strictly smaller order than $\gamma$, we can proceed by induction. By writing $x^{t}$ for $r^{t}, \rho^{t}, \theta^{t}$ and

$$
\partial^{\gamma_{l}^{i}}=\partial_{\eta}^{\beta_{l}^{i}} \partial_{r}^{k_{l}^{i}} \partial_{\theta}^{\alpha_{l}^{i}} \partial_{\rho}^{j_{l}^{i}}
$$

for the derivatives involved in (3-26), with $1 \leq i \leq 2 n$ and $1 \leq l \leq v_{i}$ (recall that, if $v_{i}=0$, the corresponding product in (3-26) is 1$)$, the induction hypothesis yields

$$
\left|e^{\left|\beta_{l}^{i}\right| r} \partial^{\gamma_{l}^{i}} x^{t}\right| \lesssim\langle t\rangle
$$

since $\partial_{\eta}^{\beta_{l}^{i}} x^{t}=\partial_{\eta}^{\beta_{l}^{i}}\left(x^{t}-x^{0}\right)$ if $\beta_{l}^{i} \neq 0$. If $n+2 \leq i \leq 2 n$ (and $v_{i} \neq 0$ ), we also have

$$
\left|e^{\left|\beta_{l}^{i}\right| r} \partial^{\gamma_{l}^{i}} \eta_{i-n-1}^{t}\right| \lesssim\langle t\rangle,
$$

unless $\partial \gamma_{l}^{i}=\partial_{\eta}^{\beta_{l}^{i}}$ with $\left|\beta_{l}^{i}\right|=1$, in which case we only have $\left|\partial^{\gamma_{l}^{i}} \eta_{i-n-1}^{t}\right| \lesssim\langle t\rangle$. By setting

$$
\mathscr{E}=\left\{n+2 \leq i \leq 2 n: \exists 1 \leq l \leq v_{i} \text { such that } \partial^{\gamma_{l}^{i}}=\partial_{\eta}^{\beta_{l}^{i}} \text { with }\left|\beta_{l}^{i}\right|=1\right\},
$$

and $N=\# \mathscr{E}$, we thus obtain

$$
|(3-26)| \lesssim e^{N r}\left|\left(\partial^{\nu} H_{p}\right)\left(\Phi^{t}\right)\right|\langle t\rangle^{|\nu|-N} \prod_{\mathscr{E}}\left|\partial^{\gamma_{l}^{i}} \eta_{i-n-1}^{t}\right| .
$$

Since the components of $H_{p}$ are polynomial of degree 2 with respect to the last $n-1$ variables, we only need to consider the case where $N \leq 2$, otherwise $v_{n+2}+\cdots+v_{2 n} \geq 3$ and $\partial^{v} H_{p} \equiv 0$. Furthermore

$$
\left|\left(\partial^{v} H_{p}\right)\left(\Phi^{t}\right)\right| \lesssim e^{-2 r^{t}}\left\langle\eta^{t}\right\rangle^{2-v_{n+2}-\cdots-v_{2 n}} \lesssim e^{-2 r^{t}}\left\langle\eta^{t}\right\rangle^{2-N} .
$$

For $N \leq 2$, we have $\left\langle\eta^{t}\right\rangle^{2-N} \lesssim\langle\eta\rangle^{2-N}+\langle t\rangle^{2-N}$ so, using that $e^{N r} e^{-2 r^{t}} \lesssim e^{-(2-N) r-2 \sigma t}$, we see that $e^{N r} e^{-2 r^{t}}\left\langle\eta^{t}\right\rangle^{2-N} \lesssim e^{-\sigma t}$ which finally implies $|(3-26)| \lesssim\langle t\rangle^{|\nu|} e^{-\sigma t} \lesssim e^{-\sigma t / 2}$. Therefore $|Y(t)| \lesssim\langle t\rangle e^{-\sigma t}$ and, by Lemma 3.7, $|X(t)|$ is bounded. The result then follows easily.

The following proposition will be important in Section $4 \mathrm{C}$ to construct and estimate phase functions.

Proposition 3.8. For all $0<\sigma<1$, there exists $R>0$ such that, for all $j, k \in \mathbb{N}_{0}, \alpha, \beta \in \mathbb{N}_{0}^{n-1}$, with the notation

$$
D_{\text {hyp }}^{j \alpha k \beta}=e^{r|\beta|} \partial_{\eta}^{\beta} \partial_{r}^{j} \partial_{\theta}^{\alpha} \partial_{\rho}^{k},
$$

(introduced before Definition 2.2) and $(l)_{+}=\max (0, l)$, we have

$$
\begin{aligned}
\left|D_{\text {hyp }}^{j \alpha k \beta}\left(r^{t}-r-2|t| p^{1 / 2}\right)\right| & \lesssim\left(e^{-r}\left\langle\eta / p^{1 / 2}\right\rangle\right)^{(2-|\beta|)_{+}} p^{-(k+|\beta|) / 2}, \\
\left|D_{\text {hyp }}^{j \alpha k \beta}\left(\theta^{t}-\theta\right)\right| & \lesssim e^{-r}\left(e^{-r}\left\langle\eta / p^{1 / 2}\right\rangle\right)^{(1-|\beta|)_{+}} p^{-(k+|\beta|) / 2}, \\
\left|D_{\text {hyp }}^{j \alpha k \beta}\left(\rho^{t}-\rho\right)\right|+\left|D_{\text {hyp }}^{j \alpha k \beta}\left(\eta^{t}-\eta\right)\right| & \lesssim\left(e^{-r}\left\langle\eta / p^{1 / 2}\right\rangle\right)^{(2-|\beta|)_{+}} p^{(1-k-|\beta|) / 2},
\end{aligned}
$$

and, for all $0<\varepsilon<1$,

$$
\left|D_{\text {hyp }}^{j \alpha k \beta}\left(\rho^{t} \mp p^{1 / 2}\right)\right| \lesssim\left(e^{-r}\left\langle\eta / p^{1 / 2}\right\rangle\right)^{(2-|\beta|)_{+}} e^{-4(1-\varepsilon)|t| p^{1 / 2}} p^{(1-k-|\beta|) / 2},
$$


uniformly with respect to $(r, \theta, \rho, \eta)$ and $t$ satisfying

$$
r>R, \quad \pm \rho>-\sigma p^{1 / 2}, \quad \pm t \geq 0 .
$$

Apart from the energy localization and the localization in $\theta$, the conditions (3-30) are the main ones that define outgoing/incoming areas according to Definition 2.10.

Note also that, if $(r, \theta, \rho, \eta)$ are restricted to a subset where $p$ belongs to a compact subset of $(0,+\infty)$, the estimates of Proposition 3.8 read

$$
\begin{aligned}
\left|D_{\text {hyp }}^{j \alpha k \beta}\left(r^{t}-r-2|t| p^{1 / 2}\right)\right|+\left|D_{\text {hyp }}^{j \alpha k \beta}\left(\rho^{t}-\rho\right)\right|+\left|D_{\text {hyp }}^{j \alpha k \beta}\left(\eta^{t}-\eta\right)\right| & \lesssim\left(e^{-r}\langle\eta\rangle\right)^{(2-|\beta|)_{+}}, \\
\left|D_{\text {hyp }}^{j \alpha k \beta}\left(\theta^{t}-\theta\right)\right| & \lesssim e^{-r}\left(e^{-r}\langle\eta\rangle\right)^{(1-|\beta|)_{+}}, \\
\left|D_{\text {hyp }}^{j \alpha k \beta}\left(\rho^{t} \mp p^{1 / 2}\right)\right| & \lesssim\left(e^{-r}\langle\eta\rangle\right)^{(2-|\beta|)_{+}} e^{-4(1-\varepsilon)|t| p^{1 / 2}} .
\end{aligned}
$$

Actually the latter estimates are equivalent to Proposition 3.8, in view of the elementary scaling properties

$$
\begin{aligned}
& \left(r^{t}, \theta^{t}\right)(r, \theta, \rho, \eta)=\left(r^{\lambda t}, \theta^{\lambda t}\right)(r, \theta, \rho / \lambda, \eta / \lambda), \\
& \left(\rho^{t}, \eta^{t}\right)(r, \theta, \rho, \eta)=\lambda\left(\rho^{\lambda t}, \eta^{\lambda t}\right)(r, \theta, \rho / \lambda, \eta / \lambda),
\end{aligned}
$$

valid for $\lambda>0$. Note that the condition (3-30) is invariant under the scaling $(t, \rho, \eta) \mapsto(\lambda t, \rho / \lambda, \eta / \lambda)$.

To prove Proposition 3.8, we need the following lemma (which will also be useful in proof of Proposition 2.16 in Section 4A).

Lemma 3.9. For all $0<\sigma<1$, there exist $R>0$ and $C>0$ such that, for all $(r, \theta, \rho, \eta)$ satisfying $(3-23)$,

$$
\left|\rho^{t} \mp p^{1 / 2}\right| \leq C e^{-|t| / C} \quad \text { for } \pm t \geq 0 .
$$

In particular, $\rho^{t} \rightarrow \pm p^{1 / 2}$ as $t \rightarrow \pm \infty$.

Proof. We consider the case where $t \geq 0$, the case of negative times being similar. Using (3-12), Proposition 3.4 and Lemma 3.5, we can choose $R$ large enough such that $\dot{\rho}^{t} \geq 0$ and

$$
\dot{\rho}^{t} \lesssim e^{-2 r^{t}}\left|\eta^{t}\right|^{2} \lesssim e^{-2 r^{t}}(|\eta|+\langle t\rangle)^{2} \lesssim e^{-2 r-2 \sigma t}(|\eta|+\langle t\rangle)^{2} \lesssim e^{-\sigma t}
$$

using the fact that $e^{-2 r}|\eta|^{2} \lesssim p$ in the last estimate. Therefore, $\rho^{t}$ has a limit as $t \rightarrow+\infty$. By the energy conservation and the estimate on $e^{-2 r^{t}}\left|\eta^{t}\right|^{2}$ given by (3-37), we have $p=\left(\rho^{t}\right)^{2}+\mathcal{O}\left(e^{-\sigma t}\right)$, which shows that $\left(\rho^{t}\right)^{2} \rightarrow p$. Since $\rho^{t}$ is nondecreasing and $\rho^{0}=\rho>-p^{1 / 2}$, the limit must be $p^{1 / 2}$. Then we get (3-36) by integrating the equation of motion for $\rho^{t}$ between $t$ and $+\infty$, namely

$$
p^{1 / 2}-\rho^{t}=\int_{t}^{\infty} \dot{\rho}^{s} d s=\int_{t}^{\infty} e^{-2 r^{s}}\left(2 q\left(r^{s}, \theta^{s}, \eta^{s}\right)-\left(\partial_{r} q_{1}\right)\left(r^{s}, \theta^{s}, \eta^{s}\right)\right) d s
$$

where, by Proposition 3.4 and Lemma 3.5, the integrand is $\mathcal{O}\left(e^{-2 r-2 \sigma s}(\langle s\rangle+\langle\eta\rangle)^{2}\right)$.

Proof of Proposition 3.8. We only need to prove (3-31), (3-32) and (3-33) with $p \in\left(\frac{1}{4}, 4\right)$ and, again, we only consider $t \geq 0$ and $\rho>-\sigma p^{1 / 2}$. We first assume that $j+|\alpha|+k+|\beta|=0$. By (3-10), Proposition 3.4 and Lemma 3.5, we have

$$
\begin{aligned}
& \left|\dot{\theta}^{t}\right| \lesssim e^{-2 r-2 \sigma t}(|\eta|+\langle t\rangle) \lesssim e^{-2 r-\sigma t}\langle\eta\rangle, \\
& \left|\dot{\eta}^{t}\right| \lesssim e^{-2 r-2 \sigma t}(|\eta|+\langle t\rangle)^{2} \lesssim e^{-2 r-\sigma t}\langle\eta\rangle^{2}
\end{aligned}
$$


hence $\left|\eta^{t}-\eta\right| \lesssim e^{-2 r}\langle\eta\rangle^{2}$ and $\left|\theta^{t}-\theta\right| \lesssim e^{-2 r}\langle\eta\rangle$. In particular, $\eta^{t}-\eta$ and $\theta^{t}-\theta$ are bounded. The motion equation for $r^{t}$ yields

$$
r^{t}-r-2 t p^{1 / 2}=2 \int_{0}^{t}\left(\rho^{s}-p^{1 / 2}\right) d s,
$$

and, using (3-36), we get $\left|r^{t}-r-2 t p^{1 / 2}\right| \lesssim 1$. The latter estimate and the boundedness $\left|\eta^{t}-\eta\right|$ imply, together with (3-38),

$$
\left|\rho^{t}-p^{1 / 2}\right| \lesssim e^{-2 r-4 t p^{1 / 2}}\langle\eta\rangle^{2} .
$$

Furthermore, since $\left|p^{1 / 2}-\rho\right|=\left|\rho^{2}-p\right| /\left|\rho+p^{1 / 2}\right| \lesssim e^{-2 r}|\eta|^{2}$, we also have $\left|\rho^{t}-\rho\right| \lesssim e^{-2 r}\langle\eta\rangle^{2}$. Putting (3-40) into (3-39), we obtain $\left|r^{t}-r-2 t p^{1 / 2}\right| \lesssim e^{-2 r}\langle\eta\rangle^{2}$ which completes the proof of (3-31), (3-32) and (3-33) for $j+|\alpha|+k+|\beta|=0$ (note that we can choose $\varepsilon=0$ in this case).

We now prove (3-32) when $j+|\alpha|+k+|\beta| \geq 1$. We first note that, by Lemma 3.5 and the boundedness of $\left|r^{t}-r-2 t p^{1 / 2}\right|$, we have

$$
\begin{aligned}
\left|D_{\text {hyp }}^{j^{\prime} \alpha^{\prime} k^{\prime} \beta^{\prime}}\left(e^{-r^{t}} \eta^{t}\right)\right| & \leq\left|D_{\text {hyp }}^{j^{\prime} \alpha^{\prime} k^{\prime} \beta^{\prime}}\left(e^{-r^{t}}\left(\eta^{t}-\eta\right)\right)\right|+\left|D_{\text {hyp }}^{j^{\prime} \alpha^{\prime} k^{\prime} \beta^{\prime}}\left(e^{-r^{t}} \eta\right)\right| \\
& \lesssim e^{-2 t p^{1 / 2}}\langle t\rangle^{j^{\prime}+\left|\alpha^{\prime}\right|+k^{\prime}+\left|\beta^{\prime}\right|}\left(e^{-r}+\left(e^{-r}|\eta|\right)^{\left(1-\left|\beta^{\prime}\right|\right)_{+}}\right) \\
& \lesssim e^{-2 t p^{1 / 2}}\langle t\rangle^{j^{\prime}+\left|\alpha^{\prime}\right|+k^{\prime}+\left|\beta^{\prime}\right|}\left(e^{-r}\langle\eta\rangle\right)^{\left(1-\left|\beta^{\prime}\right|\right)_{+}},
\end{aligned}
$$

for all $j^{\prime}+\left|\alpha^{\prime}\right|+k^{\prime}+\left|\beta^{\prime}\right| \geq 0$. By writing

$$
\theta^{t}-\theta=\int_{0}^{t} e^{-r^{s}}\left(\partial_{\eta} q\right)\left(r^{s}, \theta^{s}, e^{-r^{s}} \eta^{s}\right) d s,
$$

and using (3-41), Lemma 3.5 (more precisely, the estimates $\left|D_{\text {hyp }}^{j^{\prime \prime} \alpha^{\prime \prime} k^{\prime \prime}{ }^{t}} r^{t}\right|+\left|D_{\text {hyp }}^{j^{\prime \prime} \alpha^{\prime \prime} k^{\prime \prime} \beta^{\prime \prime}} \theta^{t}\right| \lesssim\langle t\rangle$ if $j^{\prime \prime}+$ $\left|\alpha^{\prime \prime}\right|+k^{\prime \prime}+\left|\beta^{\prime \prime}\right| \neq 0$ ), the Leibniz formula and Lemma 3.6, we obtain (3-32). We obtain similarly (3-33) and then (3-31) (also using that $\left(e^{-r}\langle\eta\rangle\right)^{2} \lesssim e^{-r}\langle\eta\rangle \lesssim 1$ ). Note that, for $r^{t}-r-2 t p^{1 / 2},(3-31)$ follows directly from (3-33) and (3-39).

Corollary 3.10. Let $V \Subset V^{\prime} \Subset \mathbb{R}^{n-1}$ be two relatively compact open subsets and let $0<\sigma<1$. There exists $R>0$ and $C>0$ such that the conditions

$$
r>R, \quad \theta \in V, \quad \pm \rho>-\sigma p^{1 / 2},
$$

imply that, for all $\pm t \geq 0$,

$$
r^{t}>r-C \text { and } \theta^{t} \in V^{\prime} .
$$

In particular, if (3-42) holds, the flow $\Phi^{t}(r, \theta, \rho, \eta)$ depend only on $p$ on $T^{*}\left((r-C,+\infty) \times V^{\prime}\right)$ for $\pm t \geq 0$.

This corollary allows us to localize the estimates of Proposition 3.8 in charts of asymptotically hyperbolic manifolds.

\section{The Hamilton-Jacobi and transport equations}

In this section, we develop the analytical tools necessary for the Isozaki-Kiada parametrix that will be constructed in Section 5. We mainly construct the phases and amplitudes needed for that parametrix, but 
also prove certain useful properties of outgoing/incoming areas, including those quoted without proof in Section 2D.

All the statements in this section will hold in a coordinate chart at infinity, associated to a fixed coordinate patch $U_{\iota}$ on the angular manifold. Thus, for notational simplicity, we will drop the corresponding index $\iota$ from the notation.

4A. Properties of outgoing, incoming and intermediate areas. Here we collect some properties of outgoing, incoming and intermediate areas which will be needed for the construction of the Isozaki-Kiada parametrix. We also prove a part of the results quoted without proofs in Section 2D, namely Propositions $2.11,2.13,2.15$ and 2.16 .

In the first proposition below, we use the classes $\mathscr{S}_{\text {hyp }}(\Omega)$ introduced in Definition 2.2.

Proposition 4.1. (i) Assume that

$$
R_{1}>R_{2}, \quad V_{1} \Subset V_{2}, \quad I_{1} \Subset I_{2}, \quad \sigma_{1}<\sigma_{2} .
$$

Then we can find $\chi_{1 \rightarrow 2}^{ \pm} \in \mathscr{S}_{\text {hyp }}\left(\Gamma^{ \pm}\left(R_{2}, V_{2}, I_{2}, \sigma_{2}\right)\right)$ such that

$$
\chi_{1 \rightarrow 2}^{ \pm} \equiv 1 \quad \text { on } \Gamma^{ \pm}\left(R_{1}, V_{1}, I_{1}, \sigma_{1}\right) \text {. }
$$

(ii) Any symbol $a \in \mathscr{S}_{\text {hyp }}\left((R,+\infty) \times V \times \mathbb{R}^{n} \cap p^{-1}(I)\right)$ can be written

$$
a=a^{+}+a^{-}, \quad \text { with } a^{ \pm} \in \mathscr{S}_{\text {hyp }}\left(\Gamma^{ \pm}\left(R, V, I, \frac{1}{2}\right)\right) .
$$

One important point in this proposition is that $\chi_{1 \rightarrow 2}^{ \pm}$and $a^{ \pm}$can be chosen in $\mathscr{S}_{\text {hyp. }}$.

Proof. (i) We may for instance choose

$$
\chi_{1 \rightarrow 2}^{ \pm}(r, \theta, \rho, \eta)=\chi_{R_{1} \rightarrow R_{2}}(r) \chi_{V_{1} \rightarrow V_{2}}(\theta) \chi_{I_{1} \rightarrow I_{2}}(p) \chi_{\sigma_{1} \rightarrow \sigma_{2}}\left( \pm \rho / p^{1 / 2}\right),
$$

with $\chi_{R_{1} \rightarrow R_{2}}, \chi_{\sigma_{1} \rightarrow \sigma_{2}} \in C^{\infty}(\mathbb{R}), \chi_{V_{1} \rightarrow V_{2}} \in C_{0}^{\infty}\left(V_{2}\right)$ and $\chi_{I_{1} \rightarrow I_{2}} \in C_{0}^{\infty}\left(I_{2}\right)$ such that

$$
\operatorname{supp}\left(\chi_{R_{1} \rightarrow R_{2}}\right) \subset\left(R_{2},+\infty\right), \quad \operatorname{supp}\left(\chi_{\sigma_{1} \rightarrow \sigma_{2}}\right) \subset\left(-\sigma_{2},+\infty\right),
$$

$$
\chi_{R_{1} \rightarrow R_{2}} \equiv 1 \quad \text { on }\left(R_{1},+\infty\right), \quad \chi_{V_{1} \rightarrow V_{2}} \equiv 1 \quad \text { on } V_{1}, \quad \chi_{I_{1} \rightarrow I_{2}} \equiv 1 \quad \text { on } I_{1}, \quad \chi_{\sigma_{1} \rightarrow \sigma_{2}} \equiv 1 \quad \text { on }\left(-\sigma_{1},+\infty\right) \text {. }
$$

Notice that $\rho / p^{1 / 2}$ is smooth on the support of $\chi_{I_{1} \rightarrow I_{2}}(p)$. The so defined $\chi_{1 \rightarrow 2}^{ \pm}$is smooth on $\mathbb{R}^{2 n}$, supported in $\Gamma^{ \pm}\left(R_{2}, V_{2}, I_{2}, \sigma_{2}\right)$, identically 1 on $\Gamma^{ \pm}\left(R_{1}, V_{1}, I_{1}, \sigma_{1}\right)$, and one easily checks that it belongs to $\mathscr{P}_{\text {hyp }}\left(\Gamma^{ \pm}\left(R_{2}, V_{2}, I_{2}, \sigma_{2}\right)\right)$, using for instance Lemma 2.4.

(ii) This is very similar to the first case. We may for instance choose

$$
a^{ \pm}(r, \theta, \rho, \eta)=a(r, \theta, \rho, \eta) \chi_{1 / 2}^{ \pm}\left(\rho / p^{1 / 2}\right),
$$

with $\chi_{1 / 2}^{ \pm} \in C^{\infty}(\mathbb{R})$ such that

$$
\chi_{1 / 2}^{+}+\chi_{1 / 2}^{-} \equiv 1, \quad \operatorname{supp}\left(\chi_{1 / 2}^{+}\right) \subset\left(-\frac{1}{2},+\infty\right), \quad \operatorname{supp}\left(\chi_{1 / 2}^{+}\right) \subset\left(-\infty, \frac{1}{2}\right) .
$$

Here again $\rho / p^{1 / 2}$ is smooth on the support of $a$ and $a^{ \pm} \in \mathscr{B}_{\mathrm{hyp}}\left(\Gamma^{ \pm}\left(R, V, I, \frac{1}{2}\right)\right)$.

By Proposition 4.1(i), $\Gamma^{ \pm}\left(R_{2}, V_{2}, I_{2}, \sigma_{2}\right)$ is a neighborhood of the closure of $\Gamma^{ \pm}\left(R_{1}, V_{1}, I_{1}, \sigma_{1}\right)$ under the assumption (4-1). In the following proposition, we make this remark more quantitative. 
Proposition 4.2. Assume (4-1). There exists $\varepsilon>0$ such that, for all $\left(r^{\prime}, \theta^{\prime}, \rho^{\prime}, \eta^{\prime}\right) \in \mathbb{R}^{2 n}$ and all $(r, \theta, \rho, \eta) \in \Gamma^{ \pm}\left(R_{1}, V_{1}, I_{1}, \sigma_{1}\right)$,

$$
\left|(r, \theta, \rho, \eta)-\left(r^{\prime}, \theta^{\prime}, \rho^{\prime}, \eta^{\prime}\right)\right| \leq \varepsilon \quad \Longrightarrow \quad\left(r^{\prime}, \theta^{\prime}, \rho^{\prime}, \eta^{\prime}\right) \in \Gamma^{ \pm}\left(R_{2}, V_{2}, I_{2}, \sigma_{2}\right) .
$$

Proof. Choose first $\varepsilon_{0}>0$ such that, if $\left|r-r^{\prime}\right|+\left|\theta-\theta^{\prime}\right| \leq \varepsilon_{0}, r^{\prime}>R_{2}$ and $\theta^{\prime} \in V_{2}$. Then, by writing

$$
q\left(r^{\prime}, \theta^{\prime}, e^{-r^{\prime}} \eta^{\prime}\right)-q\left(r^{\prime}, \theta^{\prime}, e^{-r} \eta\right)=e^{-2 r^{\prime}} q\left(r^{\prime}, \theta^{\prime}, \eta^{\prime}-\eta\right)+\left(e^{2\left(r-r^{\prime}\right)}-1\right) q\left(r^{\prime}, \theta^{\prime}, e^{-r} \eta\right),
$$

and using (3-5), (3-6) with the Taylor formula, we get

$$
\left|p\left(r^{\prime}, \theta^{\prime}, \rho^{\prime}, \eta^{\prime}\right)-p(r, \theta, \rho, \eta)\right| \leq\left|\rho^{2}-\rho^{\prime 2}\right|+C\left|\eta^{\prime}-\eta\right|^{2}+C\left(\left|r-r^{\prime}\right|+\left|\theta-\theta^{\prime}\right|\right) e^{-2 r}|\eta|^{2},
$$

where $e^{-2 r}|\eta|^{2}$ is bounded, using (3-7). Since $\rho$ is bounded too, we obtain

$$
\left|p\left(r^{\prime}, \theta^{\prime}, \rho^{\prime}, \eta^{\prime}\right)-p(r, \theta, \rho, \eta)\right| \leq C\left|(r, \theta, \rho, \eta)-\left(r^{\prime}, \theta^{\prime}, \rho^{\prime}, \eta^{\prime}\right)\right|,
$$

provided that $\left|(r, \theta, \rho, \eta)-\left(r^{\prime}, \theta^{\prime}, \rho^{\prime}, \eta^{\prime}\right)\right| \leq \varepsilon_{0}$ and therefore,

$$
\begin{aligned}
\left|p^{1 / 2}\left(r^{\prime}, \theta^{\prime}, \rho^{\prime}, \eta^{\prime}\right)-p^{1 / 2}(r, \theta, \rho, \eta)\right| & \leq C\left|(r, \theta, \rho, \eta)-\left(r^{\prime}, \theta^{\prime}, \rho^{\prime}, \eta^{\prime}\right)\right|, \\
\left|\frac{\rho^{\prime}}{p^{1 / 2}\left(r^{\prime}, \theta^{\prime}, \rho^{\prime}, \eta^{\prime}\right)}-\frac{\rho}{p^{1 / 2}(r, \theta, \rho, \eta)}\right| & \leq C\left|(r, \theta, \rho, \eta)-\left(r^{\prime}, \theta^{\prime}, \rho^{\prime}, \eta^{\prime}\right)\right|,
\end{aligned}
$$

if $\left|(r, \theta, \rho, \eta)-\left(r^{\prime}, \theta^{\prime}, \rho^{\prime}, \eta^{\prime}\right)\right|$ is small enough, using that $I_{2} \Subset(0,+\infty)$. The conclusion is then easy.

Similarly to (2-54), we fix $V_{0} \subset \mathbb{R}^{n-1}$ a relatively compact open subset of $\psi_{\iota}\left(U_{\iota}\right)$ and define

$$
R(\varepsilon)=1 / \varepsilon, \quad V_{\varepsilon}=\left\{\theta \in \mathbb{R}^{n-1}: \operatorname{dist}\left(\theta, V_{0}\right)<\varepsilon^{2}\right\} .
$$

In the sequel, we shall need very often the following result on strongly outgoing/incoming areas (see Propositions 4.8, 4.14 and Lemmas 4.11, 4.16). This will for instance be the case when we use Taylor's formula and want to guarantee that the whole segment between two points of a strongly outgoing/incoming area is still contained in such an area.

Proposition 4.3. For all $M>0$, there exist $\varepsilon_{M}>0$ and $C_{M}>1$ such that, for all $0<\varepsilon \leq \varepsilon_{M}$, the following holds: if

$$
(r, \theta, \rho, \eta) \in \Gamma_{\mathrm{s}}^{ \pm}(\varepsilon)
$$

and

$$
r^{\prime}-r \geq-M, \quad\left|\theta^{\prime}-\theta\right|<M \varepsilon^{2}, \quad\left|\rho^{\prime}-\rho\right|<M \varepsilon^{2}, \quad\left|\eta^{\prime}-\eta\right|<M \varepsilon e^{1 / \varepsilon},
$$

then, for all $0 \leq s \leq 1$,

$$
\left(r^{\prime}, \theta^{\prime}, \rho^{\prime}, s \eta^{\prime}\right) \in \Gamma_{\mathrm{s}}^{ \pm}\left(C_{M} \varepsilon\right) .
$$

In particular, $\left(r^{\prime}, \theta^{\prime}, \rho^{\prime}, 0\right) \in \Gamma_{\mathrm{s}}^{ \pm}\left(C_{M} \varepsilon\right)$.

Remark. There should not be any confusion between the interpolation parameter $0 \leq s \leq 1$ and the subscript $_{\mathrm{s}}$, which refers to strongly outgoing/incoming areas (and which are independent of $s$ ). 
Proof. Using (2-56) and (4-2), we first note the existence of $M^{\prime}>0$ such that, for all $0<\varepsilon<\frac{1}{4}$, if (4-4) and (4-5) hold then

$$
\left|p\left(r^{\prime}, \theta^{\prime}, \rho^{\prime}, s \eta^{\prime}\right)-p(r, \theta, \rho, \eta)\right| \leq M^{\prime} \varepsilon^{2},
$$

using in particular that $s \eta^{\prime}-\eta=s\left(\eta^{\prime}-\eta\right)+(s-1) \eta$. If $C_{M}$ is large enough and $0<\varepsilon C_{M}<\frac{1}{4}$, we obtain

$$
0<\frac{1}{4}-C_{M} \varepsilon<\frac{1}{4}-\varepsilon-M^{\prime} \varepsilon^{2} \leq p\left(r^{\prime}, \theta^{\prime}, \rho^{\prime}, s \eta^{\prime}\right) \leq 4+\varepsilon+M^{\prime} \varepsilon^{2}<4+C_{M} \varepsilon .
$$

If $0<\varepsilon \leq \varepsilon_{M}$ with $\varepsilon_{M}$ small enough, then $p\left(r^{\prime}, \theta^{\prime}, \rho^{\prime}, s \eta^{\prime}\right) / p(r, \theta, \rho, \eta)=1+\mathcal{O}\left(\varepsilon^{2}\right)$ so that

$$
\frac{ \pm \rho^{\prime}}{p\left(r^{\prime}, \theta^{\prime}, \rho^{\prime}, s \eta^{\prime}\right)^{1 / 2}}=\frac{ \pm \rho}{p(r, \theta, \rho, \eta)^{1 / 2}} \frac{p(r, \theta, \rho, \eta)^{1 / 2}}{p\left(r^{\prime}, \theta^{\prime}, \rho^{\prime}, s \eta^{\prime}\right)^{1 / 2}} \pm \frac{\rho^{\prime}-\rho}{p\left(r^{\prime}, \theta^{\prime}, \rho^{\prime}, s \eta^{\prime}\right)^{1 / 2}}>1-\left(C_{M} \varepsilon\right)^{2},
$$

by possibly increasing $C_{M}$. In addition, $\operatorname{dist}\left(\theta, V_{0}\right) \leq\left|\theta^{\prime}-\theta\right|+\operatorname{dist}\left(\theta, V_{0}\right)<\left(C_{M} \varepsilon\right)^{2}$, by possibly increasing $C_{M}$ again and decreasing $\varepsilon_{M}$. Finally, $r^{\prime} \geq r-M>e^{1 / \varepsilon}-M>e^{1 / C_{M} \varepsilon}$, for all $0<\varepsilon \leq \varepsilon_{M}$ by possibly decreasing $\varepsilon_{M}$ again, so $\left(r^{\prime}, \theta^{\prime}, \rho^{\prime}, s \eta^{\prime}\right) \in \Gamma_{\mathrm{s}}^{ \pm}\left(C_{M} \varepsilon\right)$.

We can now prove Proposition 2.13, which states that one can reach a strongly outgoing (incoming) area from an outgoing (incoming) one in finite time, along the geodesic flow.

Proof of Proposition 2.13. We consider only the outgoing case. With no loss of generality, we may assume that $0<\sigma<1$. By choosing $R \geq R_{\sigma}^{\prime}$ large enough, we can use Proposition 3.4 and Corollary 3.10. By Proposition 3.4, we have $r_{t} \geq r+c t-C$ for some $C, c>0$, hence $r_{t}>R(\varepsilon)$ for all $t \geq t_{R, \varepsilon}$, provided

$$
c t_{R, \varepsilon}-C+R>R(\varepsilon) \text {. }
$$

By Proposition 3.8, we have $\left|\theta^{t}-\theta\right| \lesssim e^{-r}$ hence $\theta^{t} \in V_{\varepsilon}$, for $\varepsilon$ small enough and all $t \geq 0$, since $e^{-1 / \varepsilon} \ll \varepsilon^{2}$. Using (3-33) and the energy conservation, we shall have $\rho^{t} / p^{1 / 2}\left(r^{t}, \theta^{t}, \rho^{t}, \eta^{t}\right)>1-\varepsilon^{2}$ provided for instance that

$$
e^{-p^{1 / 2} t_{R, \varepsilon}} \leq \varepsilon^{3}
$$

with $\varepsilon$ small enough. Choosing $t_{R, \varepsilon}$ so that (4-6) and (4-7) hold, we get the result.

We conclude this part with an explicit construction for cutoffs.

In Section 5, we will need a result similar to Proposition 4.1(i). This is the purpose of the following result.

Proposition 4.4. We can find $0<v<1$ and a family of cutoffs $\chi_{\varepsilon^{2} \rightarrow \varepsilon}^{ \pm} \in \mathscr{Y}_{\mathrm{hyp}}\left(\Gamma_{\mathrm{s}}^{ \pm}\left(\varepsilon^{1+v}\right)\right)$, defined for all $\varepsilon$ small enough, such that

$$
\chi_{\varepsilon^{2} \rightarrow \varepsilon}^{ \pm}=1 \text { on } \Gamma_{\mathrm{s}}^{ \pm}\left(\varepsilon^{2}\right)
$$

and, uniformly on $\mathbb{R}^{2 n}$,

$$
\left.\left|e^{-2 r}\right| \eta\right|^{j} \partial_{r, \theta, \rho, \eta} \chi_{\varepsilon^{2} \rightarrow \varepsilon}^{ \pm}|+| e^{-2 r}|\eta|^{2} \partial_{\rho, \eta} \partial_{r, \theta} \chi_{\varepsilon^{2} \rightarrow \varepsilon}^{ \pm} \mid \lesssim \varepsilon^{1 / 2}, \quad j=1,2 .
$$

That we can find, for each $\varepsilon, \chi_{\varepsilon^{2} \rightarrow \varepsilon}^{ \pm} \in \mathscr{I}_{\text {hyp }}\left(\Gamma_{\mathrm{s}}^{ \pm}\left(\varepsilon^{1+v}\right)\right)$ satisfying (4-8) would follow directly from Proposition 4.1. The important additional point here is the control with respect to $\varepsilon$ given by (4-9). Note also that the power $\frac{1}{2}$ is essentially irrelevant: we only mean that the left-hand side of (4-9) is uniformly small as $\varepsilon \rightarrow 0$. This rather technical point will only be used in Section 5 to globalize suitably certain phase functions. 
Proof. For $0<\delta<1$ to be chosen later, we consider the characteristic functions $\bar{\chi}_{\varepsilon^{1+\delta}}^{I}$ and $\bar{\chi}_{\varepsilon^{2+\delta}}^{V}$ of $\left(\frac{1}{4}-\varepsilon^{1+\delta}, 4+\varepsilon^{1+\delta}\right)$ and $V+B\left(0, \varepsilon^{2+\delta}\right)$ respectively. Choose $\zeta^{I} \in C_{0}^{\infty}(\mathbb{R}), \zeta^{V} \in C_{0}^{\infty}\left(\mathbb{R}^{n-1}\right)$ both equal to 1 near 0 , such that $\int \zeta^{I}=\int \zeta^{V}=1$ and set

$$
\chi_{\varepsilon^{1+\delta}}^{I}(\lambda)=\int \bar{\chi}_{\varepsilon^{1+\delta}}^{I}(\mu) \zeta^{I}\left(\frac{\lambda-\mu}{\varepsilon^{1+2 \delta}}\right) \varepsilon^{-1-2 \delta} d \mu, \quad \chi_{\varepsilon^{2+\delta}}^{V}(\theta)=\int \bar{\chi}_{\varepsilon^{2+\delta}}^{V}(\vartheta) \zeta^{V}\left(\frac{\theta-\vartheta}{\varepsilon^{2+2 \delta}}\right) \varepsilon^{-(n-1)(2+2 \delta)} d \vartheta .
$$

One then easily checks that, if $\varepsilon$ is small enough,

$$
\begin{array}{ll}
\chi_{\varepsilon^{1+\delta}}^{I} \equiv 1 \text { on }\left(\frac{1}{4}-\varepsilon^{2}, 4+\varepsilon^{2}\right), & \chi_{\varepsilon^{2+\delta}}^{V}(\theta)=1 \text { if } \operatorname{dist}(\theta, V)<\varepsilon^{4}, \\
\chi_{\varepsilon^{1+\delta}}^{I} \equiv 0 \text { outside }\left(\frac{1}{4}-\varepsilon^{1+\frac{\delta}{4}}, 4+\varepsilon^{1+\frac{\delta}{4}}\right), & \chi_{\varepsilon^{2+\delta}}^{V}(\theta)=0 \text { if } \operatorname{dist}(\theta, V) \geq \varepsilon^{2+\frac{\delta}{2}} .
\end{array}
$$

Choosing $\omega \in C^{\infty}(\mathbb{R})$ supported in $\left(\frac{1}{4}, \infty\right)$ such that $\omega=1$ near $\left[\frac{1}{3}, \infty\right)$, we now define

$$
\chi_{\varepsilon^{2} \rightarrow \varepsilon}^{ \pm}(r, \theta, \rho, \eta)=\omega\left(\frac{r}{R\left(\varepsilon^{3 / 2}\right)}\right) \chi_{\varepsilon^{2+\delta}}^{V}(\theta) \chi_{\varepsilon^{1+\delta}}^{I}(p) \omega( \pm \rho) \zeta^{I}\left(e^{-2 r} \frac{|\eta|^{2}}{\varepsilon^{4-\delta}}\right) .
$$

On the support of $\chi_{\varepsilon^{1+\delta}}^{I}(p)$, we have $\rho^{2} \geq \frac{1}{4}-\mathcal{O}(\varepsilon)$ so the factor $\omega( \pm \rho)$ only determines the sign of $\rho$. By (2-55) and (2-56), one sees that (4-8) holds with $v=\delta / 2$, if $\varepsilon$ is small enough. Furthermore, $\chi_{\varepsilon^{2} \rightarrow \varepsilon}^{ \pm}$ is supported in $\Gamma_{\mathrm{s}}^{ \pm}\left(\varepsilon^{1+v}\right)$ and belongs to $\mathscr{B}_{\mathrm{hyp}}\left(\Gamma_{\mathrm{s}}^{ \pm}\left(\varepsilon^{1+v}\right)\right)$.

We prove (4-9). Since $e^{-2 r}|\eta|^{2} \lesssim \varepsilon^{4-\delta}$ on the support of $\chi_{\varepsilon^{2} \rightarrow \varepsilon}^{ \pm}$, the first-order derivatives satisfy

$$
\begin{aligned}
& \left|\partial_{r} \chi_{\varepsilon^{2} \rightarrow \varepsilon}^{ \pm}\right| \lesssim R\left(\varepsilon^{3 / 2}\right)^{-1}+e^{-2 r}|\eta|^{2}\left(\varepsilon^{-1-2 \delta}+\varepsilon^{-4+\delta}\right) \lesssim 1, \\
& \left|\partial_{\rho} \chi_{\varepsilon^{2} \rightarrow \varepsilon}^{ \pm}\right| \lesssim \varepsilon^{-1-2 \delta}, \\
& \left|\partial_{\theta} \chi_{\varepsilon^{2} \rightarrow \varepsilon}^{ \pm}\right| \lesssim \varepsilon^{-2-2 \delta}+\varepsilon^{-1-2 \delta} e^{-2 r}|\eta|^{2} \lesssim \varepsilon^{-2-2 \delta}, \\
& \left|\partial_{\eta} \chi_{\varepsilon^{2} \rightarrow \varepsilon}^{ \pm}\right| \lesssim e^{-2 r}|\eta|\left(\varepsilon^{-1-2 \delta}+\varepsilon^{-4+\delta}\right) \ll e^{-\varepsilon^{-1 / 2}},
\end{aligned}
$$

using the fact that $e^{-2 r}|\eta| \lesssim e^{-r} \leq e^{-\varepsilon^{-1}}$ for the last estimate. Similarly

$$
\left|\partial_{\rho} \partial_{r, \theta} \chi_{\varepsilon^{2} \rightarrow \varepsilon}^{ \pm}\right| \lesssim \varepsilon^{-2-2 \delta} \times \varepsilon^{-1-2 \delta}=\varepsilon^{-3-4 \delta}, \quad\left|\partial_{\eta} \partial_{r, \theta} \chi_{\varepsilon^{2} \rightarrow \varepsilon}^{ \pm}\right| \lesssim e^{-\varepsilon^{-1 / 2}} .
$$

Since $e^{-2 r}|\eta|^{2} e^{-3-4 \delta} \lesssim \varepsilon^{1-5 \delta}$ and $e^{-2 r}|\eta| \ll e^{-\varepsilon^{-1 / 2}}$, the result follows with $\delta=\frac{1}{10}$ (hence with $v=\frac{1}{20}$ ).

We finally consider the statements involving intermediate areas.

Proof of Proposition 2.15. By (2-58) and (2-59), we can find $\chi_{-\infty}, \chi_{+\infty} \in C^{\infty}(\mathbb{R})$ and

$$
\chi_{l} \in C_{0}^{\infty}\left(-\sigma_{l+1},-\sigma_{l-1}\right),
$$

for $1 \leq l \leq L-1$, such that

$$
\operatorname{supp}\left(\chi_{-\infty}\right) \subset\left(-\infty,-\sigma_{L-1}\right), \quad \operatorname{supp}\left(\chi_{+\infty}\right) \in\left(1-\varepsilon^{2},+\infty\right) \quad \text { and } \quad \chi_{+\infty}+\sum_{l=1}^{L-1} \chi_{l}+\chi_{-\infty} \equiv 1 \text { on } \mathbb{R} .
$$

This simply relies on the overlapping property of the intervals in (2-59). We then obtain the result by considering 


$$
\begin{aligned}
a_{\mathrm{s}}^{ \pm}(r, \theta, \rho, \eta) & =a^{ \pm}(r, \theta, \rho, \eta) \chi_{+\infty}\left( \pm \rho / p^{1 / 2}\right), \\
a_{l, \text { inter }}^{ \pm}(r, \theta, \rho, \eta) & =a^{ \pm}(r, \theta, \rho, \eta) \chi_{l}\left( \pm \rho / p^{1 / 2}\right), \quad 1 \leq l \leq L-2, \\
a_{L-1, \text { inter }}^{ \pm}(r, \theta, \rho, \eta) & =a^{ \pm}(r, \theta, \rho, \eta)\left(\chi_{L-1}+\chi_{-\infty}\right)\left( \pm \rho / p^{1 / 2}\right) .
\end{aligned}
$$

since, in the definition of $a_{L-1, \text { inter }}^{ \pm}$, the cutoff guarantees that $\pm \rho / p^{1 / 2}<-\sigma_{L-2}$ and $a^{ \pm}$that $\pm \rho / p^{1 / 2}>$ $-\frac{1}{2}=-\sigma_{L}$.

Proof of Proposition 2.16. We consider the outgoing case, the incoming one being similar. Using Corollary 3.10, we may assume that, if $\varepsilon$ is small enough, (3-22) holds for any initial condition such that $r>R(\varepsilon), \theta \in V$ and $\rho \geq-\frac{1}{2} p^{1 / 2}$. In particular $t \mapsto \rho^{t}$ is nondecreasing for $t \geq 0$. Assume that $\frac{1}{2} \leq \rho / p^{1 / 2} \leq 1-(\varepsilon / 2)^{2}$ and set

$$
t_{\varepsilon}=t_{\varepsilon}(r, \theta, \rho, \eta):=\sup \left\{t \geq 0: \frac{\rho^{s}}{p^{1 / 2}}<\frac{\rho}{p^{1 / 2}}+\varepsilon^{4} \text { for all } s \in[0, t]\right\} .
$$

Notice that $t_{\varepsilon}$ is finite by Lemma 3.9 and that $\rho^{t_{\varepsilon}}=\rho+p^{1 / 2} \varepsilon^{4}$. If $1-(\varepsilon / 2)^{2}+\varepsilon^{4} \geq \frac{1}{2}$, we have $\left|\rho^{t} / p^{1 / 2}\right| \leq 1-(\varepsilon / 2)^{2}+\varepsilon^{4}$ on $\left[0, t_{\varepsilon}\right)$. Thus, if $\varepsilon$ is small enough (independent of $(r, \theta, \rho, \eta)$ ), we have $\left(\rho^{t}\right)^{2} / p \leq 1-(\varepsilon / 2)^{2}$ for all $t \in\left[0, t_{\varepsilon}\right)$ and then, by (3-22) again, we have $\dot{\rho}^{t} \geq(\varepsilon / 2)^{2} p$ on $\left[0, t_{\varepsilon}\right]$, so

$$
\rho^{t_{\varepsilon}}-\rho \geq(\varepsilon / 2)^{2} p t_{\varepsilon} .
$$

This shows that $t_{\varepsilon} \leq \varepsilon^{4} /(\varepsilon / 2)^{2} p=4 \varepsilon^{2} / p$. Then, for $\varepsilon$ small enough such that $4 \varepsilon^{2} / p \leq \underline{t}$ for all $(r, \theta, \rho, \eta)$ in

$$
\left\{(r, \theta, \rho, \eta) \in \mathbb{R}^{2 n}: r>R(\varepsilon), \theta \in V, p \in I(\varepsilon),-\frac{1}{2} \leq \rho / p^{1 / 2} \leq 1-(\varepsilon / 2)^{2}\right\},
$$

and with $\delta=\varepsilon^{4} / 2$, we have $\rho^{t}-\rho \geq 2 \delta p^{1 / 2}$ for all $t \geq \underline{t}$. This implies (2-62) since, for any choice of $\sigma_{0}, \ldots, \sigma_{L}$ and any $l, \Gamma_{\text {inter }}^{ \pm}(\varepsilon, \delta ; l)$ is contained in $(4-10)$.

4B. Hyperbolic long/short-range symbols. In this short subsection, we introduce the definitions of short/long-range hyperbolic symbols which will be useful for the resolution of transport equations in Section 4E. We prove in passing Proposition 4.6 below which will be used at several places, in particular in Section 4C.

Definition 4.5. A smooth function $a_{ \pm}$on $\Gamma_{\mathrm{s}}^{ \pm}(\varepsilon)$ is said to be of hyperbolic short range if

$$
\left|\partial_{r}^{j} \partial_{\theta}^{\alpha} \partial_{\rho}^{k} \partial_{\eta}^{\beta} a_{ \pm}(r, \theta, \rho, \eta)\right| \lesssim\langle r-\log \langle\eta\rangle\rangle^{-\tau-1-j}, \quad(r, \theta, \rho, \eta) \in \Gamma_{\mathrm{s}}^{ \pm}(\varepsilon),
$$

and of hyperbolic long range if

$$
\left|\partial_{r}^{j} \partial_{\theta}^{\alpha} \partial_{\rho}^{k} \partial_{\eta}^{\beta} a_{ \pm}(r, \theta, \rho, \eta)\right| \lesssim\langle r-\log \langle\eta\rangle\rangle^{-\tau-j}, \quad(r, \theta, \rho, \eta) \in \Gamma_{\mathrm{s}}^{ \pm}(\varepsilon) .
$$

Notice that in this definition, we do not assume that $a \in \mathscr{S}_{\mathrm{hyp}}\left(\Gamma_{\mathrm{s}}^{ \pm}(\varepsilon)\right)$. However, this will be the case in the applications and we now give a simple criterion to check that a symbol $a \in \mathscr{B}_{\text {hyp }}\left(\Gamma_{\mathrm{s}}^{ \pm}(\varepsilon)\right)$ is of hyperbolic short/long range.

For $\varepsilon$ small enough, by restricting $a$ to a smaller area $\Gamma_{\mathrm{s}}^{ \pm}(\varepsilon / C)$, with $C>1$ large enough (or to $\Gamma_{\mathrm{s}}^{ \pm}\left(\varepsilon^{2}\right)$, $\Gamma_{\mathrm{s}}^{ \pm}\left(\varepsilon^{3}\right)$ as it will be the case in the applications), using Lemma 2.4 and Proposition 4.3, we have

$$
a(r, \theta, \rho, \eta)=a(r, \theta, \rho, 0)+\int_{0}^{1}\left(\partial_{\xi} \tilde{a}\right)(r, \theta, \rho, s \xi)_{\mid \xi=e^{-r} \eta} d s \cdot e^{-r} \eta
$$


where $\tilde{a}$ belongs to $C_{b}^{\infty}\left(F_{\mathrm{hyp}}\left(\Gamma_{\mathrm{s}}^{ \pm}(\varepsilon)\right)\right)$ and $(r, \theta, \rho, s \eta) \in \Gamma_{\mathrm{s}}^{ \pm}(\varepsilon)$ if $(r, \theta, \rho, \eta) \in \Gamma_{\mathrm{s}}^{ \pm}(\varepsilon / C)$. Since

$$
\left|\partial_{r}^{j} \partial_{\eta}^{\beta} e^{-r} \eta\right| \lesssim\langle r-\log \langle\eta\rangle\rangle^{-N} \quad \text { for all } N>0 \text { and }(r, \theta, \rho, \eta) \in \Gamma_{\mathrm{s}}^{ \pm}(\varepsilon),
$$

we obtain that, for $a \in \mathscr{B}_{\mathrm{hyp}}\left(\Gamma_{\mathrm{s}}^{ \pm}(\varepsilon)\right)$,

$a$ is of hyperbolic short/long range in $\Gamma_{\mathrm{s}}^{ \pm}(\varepsilon / C) \Longleftrightarrow a_{\mid \eta=0}$ is of usual short/long range

in the sense that

$$
\left|\left(\partial_{r}^{j} \partial_{\theta}^{\alpha} \partial_{\rho}^{k} a\right)(r, \theta, \rho, 0)\right| \lesssim\langle r\rangle^{-\tau-j} \quad \text { for }(r, \theta, \rho, 0) \in \Gamma_{\mathrm{s}}^{ \pm}(\varepsilon),
$$

in the long-range case (recall that $0<\tau \leq 1$ ) and

$$
\left|\left(\partial_{r}^{j} \partial_{\theta}^{\alpha} \partial_{\rho}^{k} a\right)(r, \theta, \rho, 0)\right| \lesssim\langle r\rangle^{-\tau-1-j} \quad \text { for }(r, \theta, \rho, 0) \in \Gamma_{\mathrm{s}}^{ \pm}(\varepsilon),
$$

in the short-range case.

To calculate $a_{\mid \eta=0}$ in some applications, we shall use the following elementary result.

Proposition 4.6. For all $r>0$, all $\theta \in \mathbb{R}^{n-1}$ and all $\pm \rho>0$, we have, for all $\pm t \geq 0$,

$$
\begin{aligned}
\left(r^{t}, \theta^{t}, \rho^{t}, \eta^{t}\right)_{\mid \eta=0} & =(r+2 t \rho, \theta, \rho, 0), \\
\partial_{\eta}\left(r^{t}, \theta^{t}, \rho^{t}, \eta^{t}\right)_{\mid \eta=0} & =\left(0, \int_{0}^{t} e^{-2 r-4 s \rho} \operatorname{hess}_{\eta}[q](r+s \rho, \theta) d s, 0, \text { Id }\right) .
\end{aligned}
$$

where hess ${ }_{\eta}[q](r, \theta)$ is the Hessian matrix of $q$ with respect to $\eta$ (which is independent of $\eta$ ).

Proof. One simply checks that the right-hand side of (4-15) is a solution to (3-10) (with $w(r)=e^{-2 r}$ ) for $\pm t \geq 0$. Applying then $\partial_{\eta}$ to (3-10), one sees easily as well that the right-hand side of (4-16) is a solution to the corresponding system.

Remark. If $\varepsilon$ is small enough then, on $\Gamma_{\mathrm{s}}^{ \pm}(\varepsilon)$, we have

$$
r-\log \langle\eta\rangle \geq 0 \text {. }
$$

In particular, in this region, $\langle r-\log \langle\eta\rangle\rangle$ is equivalent to the weight

$$
\langle r-\log \langle\eta\rangle\rangle_{+}:=\max (1, r-\log \langle\eta\rangle)
$$

which was introduced by Froese and Hislop [1989]. For the study of global in time estimates, which we hope to consider in a future work, the resolvent estimates proved in [Bouclet 2006] suggest that the hyperbolic short/long-range conditions (4-11)/(4-12) would play the same role as the usual Euclidean short/long-range conditions used in [Bouclet and Tzvetkov 2008].

4C. The Hamilton-Jacobi equation. We now use the results of Section 3B to solve the time-independent Hamilton-Jacobi equations giving the phases of the Isozaki-Kiada parametrix.

Lemma 4.7. There exists $0<\varepsilon_{0}<1$ such that, for all $0<\varepsilon \leq \varepsilon_{0}$ and all $\pm t \geq 0$, the map

$$
\Psi_{t}^{ \pm}:(r, \theta, \rho, \eta) \mapsto\left(r, \theta, \rho^{t}, \eta^{t}\right)
$$

is a diffeomorphism from $\Gamma_{\mathrm{s}}^{ \pm}(\varepsilon)$ onto its range and

$$
\Gamma_{\mathrm{s}}^{ \pm}\left(\varepsilon^{3}\right) \subset \Psi_{t}^{ \pm}\left(\Gamma_{\mathrm{s}}^{ \pm}(\varepsilon)\right) \quad \text { for all } \pm t \geq 0 .
$$




\section{Proof. See Appendix A.}

The power $\varepsilon^{3}$ in (4-18) is not very important. It is only a rough explicit quantitative bound for the size of a strongly outgoing area contained in $\Psi_{t}^{+}\left(\Gamma_{\mathrm{s}}^{+}(\varepsilon)\right)$ for all $t \geq 0$ (or a strongly incoming area contained in $\Psi_{t}^{-}\left(\Gamma_{\mathrm{s}}^{-}(\varepsilon)\right)$ for all $\left.t \leq 0\right)$.

The components of the inverse map $\left(\Psi_{t}^{ \pm}\right)^{-1}$ are of the form $\left(r, \theta, \rho_{t}, \eta_{t}\right)$ with

$$
\rho_{t}=\rho_{t}(r, \theta, \rho, \eta), \quad \eta_{t}=\eta_{t}(r, \theta, \rho, \eta) .
$$

Here we omit the \pm dependence for notational simplicity. We thus have

$$
\rho^{t}\left(r, \theta, \rho_{t}, \eta_{t}\right)=\rho, \quad \eta^{t}\left(r, \theta, \rho_{t}, \eta_{t}\right)=\eta,
$$

at least for all $(r, \theta, \rho, \eta) \in \Gamma_{\mathrm{s}}^{ \pm}\left(\varepsilon_{0}^{3}\right)$ and $\pm t \geq 0$.

Remark. It follows from the proof of Lemma 4.7 and the scaling properties (3-34), (3-35) that $\Psi_{t}^{ \pm}$is actually a diffeomorphism from the cone generated by $\Gamma_{\mathrm{s}}^{ \pm}\left(\varepsilon_{0}\right)$ onto its range, the latter range containing the cone generated by $\Gamma_{\mathrm{s}}^{ \pm}\left(\varepsilon_{0}^{3}\right)$. Therefore $\left(\rho_{t}, \eta_{t}\right)$ is actually the restriction to $\Gamma_{\mathrm{s}}^{ \pm}\left(\varepsilon_{0}^{3}\right)$ of a map defined on the cone generated by $\Gamma_{\mathrm{s}}^{ \pm}\left(\varepsilon_{0}^{3}\right)$ and, using (3-35), we have

$$
\left(\rho_{t}, \eta_{t}\right)(r, \theta, \lambda \rho, \lambda \eta)=\lambda\left(\rho_{\lambda t}, \eta_{\lambda t}\right)(r, \theta, \rho, \eta) \quad \text { if } \pm t \geq 0 \text { and }(r, \theta, \rho, \eta) \in \Gamma_{\mathrm{s}}^{ \pm}\left(\varepsilon_{0}^{3}\right),
$$

for all $\lambda>0$.

Proposition 4.8. There exists $\varepsilon_{1} \leq \varepsilon_{0}^{3}$ such that, for all $j, k \in \mathbb{N}_{0}, \alpha, \beta \in \mathbb{N}_{0}^{n-1}$,

$$
\left|D_{\text {hyp }}^{j \alpha k \beta}\left(\rho_{t}-\rho\right)\right|+\left|D_{\text {hyp }}^{j \alpha k \beta}\left(\eta_{t}-\eta\right)\right| \lesssim 1, \quad(r, \theta, \rho, \eta) \in \Gamma_{\mathrm{s}}^{ \pm}\left(\varepsilon_{1}\right), \quad \pm t \geq 0 .
$$

In addition, if $(r, \theta, \rho, 0) \in \Gamma_{\mathrm{s}}^{ \pm}\left(\varepsilon_{1}\right)$, we have

$$
\begin{array}{r}
\left(\rho_{t}, \eta_{t}\right)_{\mid \eta=0}=(\rho, 0), \\
\partial_{\eta}\left(\rho_{t}-\rho, \eta_{t}-\eta\right)_{\mid \eta=0}=(0,0) .
\end{array}
$$

Proof. By (4-18), any $(r, \theta, \rho, \eta) \in \Gamma_{\mathrm{s}}^{ \pm}\left(\varepsilon_{0}^{3}\right)$ can be written as $\Psi_{t}^{ \pm}(r, \theta, \tilde{\rho}, \tilde{\eta})$ with $(r, \theta, \tilde{\rho}, \tilde{\eta}) \in \Gamma_{\mathrm{s}}^{ \pm}\left(\varepsilon_{0}\right)$. Hence

$$
\sup _{\Gamma_{\mathrm{s}}^{ \pm}\left(\varepsilon_{0}^{3}\right)}\left|\rho_{t}-\rho\right|+\left|\eta_{t}-\eta\right| \leq \sup _{\Gamma_{\mathrm{s}}^{ \pm}\left(\varepsilon_{0}\right)}\left|\tilde{\rho}-\rho^{t}(\tilde{r}, \tilde{\theta}, \tilde{\rho}, \tilde{\eta})\right|+\left|\tilde{\eta}-\eta^{t}(\tilde{r}, \tilde{\theta}, \tilde{\rho}, \tilde{\eta})\right| .
$$

By (3-31), the right-hand side is bounded, so we obtain (4-21) for $j+|\alpha|+k+|\beta|=0$. Then, for $\varepsilon$ small enough, using Propositions 3.8 and 4.6, we remark that, for $(r, \theta, \rho, \eta) \in \Gamma_{\mathrm{s}}^{ \pm}(\varepsilon)$,

$$
\left|\partial_{\rho, \eta}\left(\rho^{t}-\rho, \eta^{t}-\eta\right)\right| \leq \int_{0}^{1}\left|\partial_{\eta} \partial_{\rho, \eta}\left(\rho^{t}, \eta^{t}\right)(r, \theta, \rho, s \eta)\right| d s|\eta| \lesssim\left|e^{-r} \eta\right| \lesssim \varepsilon,
$$

since, by Proposition 4.3, $(r, \theta, \rho, s \eta) \in \Gamma_{\mathrm{s}}^{ \pm}\left(\varepsilon_{0}\right)$ if $(r, \theta, \rho, \eta) \in \Gamma_{\mathrm{s}}^{ \pm}(\varepsilon)$ and $\varepsilon$ is small enough. Therefore, if $\varepsilon$ is small enough,

$$
\left|\partial_{\rho, \eta}\left(\rho^{t}, \eta^{t}\right)-\operatorname{Id}_{n}\right| \leq \frac{1}{2} \quad \text { on } \quad \Gamma_{\mathrm{s}}^{ \pm}(\varepsilon)
$$


for all $\pm t \geq 0$. Here $|\cdot|$ is a matrix norm. We can now prove (4-21) when $j+|\alpha|+k+|\beta| \geq 1$. Assume first that $D_{\text {hyp }}^{j \alpha k \beta}=e^{r} \partial_{\eta}^{\beta}$, with $|\beta|=1$, and set for simplicity

$$
\Xi_{t}(r, \theta, \rho, \eta)=\left(\rho_{t}, \eta_{t}\right)(r, \theta, \rho, \eta), \quad \Xi^{t}(r, \theta, \tilde{\rho}, \tilde{\eta})=\left(\rho^{t}, \eta^{t}\right)(r, \theta, \tilde{\rho}, \tilde{\eta}), \quad \Xi=(\rho, \eta),
$$

when $(r, \theta, \rho, \eta) \in \Gamma_{\mathrm{s}}^{ \pm}\left(\varepsilon^{3}\right),(r, \theta, \tilde{\rho}, \tilde{\eta}) \in \Gamma_{\mathrm{s}}^{ \pm}(\varepsilon)$ and $\pm t \geq 0$. Applying $e^{r} \partial_{\eta}^{\beta}$ to (4-19), we get

$$
\left(\partial_{\tilde{\rho}, \tilde{\eta}} \Xi^{t}\right)\left(r, \theta, \Xi_{t}\right) e^{r} \partial_{\eta}^{\beta} \Xi_{t}=\left(0, e^{r} \partial_{\eta}^{\beta} \eta\right)=e^{r} \partial_{\eta}^{\beta} \Xi,
$$

and using that $\left(\partial_{\tilde{\rho}, \tilde{\eta}} \Xi^{t}\right) \partial_{\eta}^{\beta} \Xi=\partial_{\tilde{\eta}}^{\beta} \Xi^{t}$, we obtain

$$
\left(\partial_{\tilde{\rho}, \tilde{\eta}} \Xi^{t}\right)\left(r, \theta, \Xi_{t}\right) e^{r} \partial_{\eta}^{\beta}\left(\Xi_{t}-\Xi\right)=e^{r}\left(\partial_{\tilde{\eta}}\left(\Xi-\Xi^{t}\right)\right)_{\mid\left(r, \theta, \Xi_{t}\right)},
$$

where the right-hand side is bounded, by (3-31). Using (4-24), we see that $e^{r} \partial_{\eta}^{\alpha}\left(\Xi_{t}-\Xi\right)$ is bounded on $\Gamma_{\mathrm{s}}^{ \pm}\left(\varepsilon_{1}\right)$ for $\pm t \geq 0$, by choosing $\varepsilon_{1} \leq \varepsilon_{0}^{3}$ and such that (4-24) holds. The other first-order derivatives are treated similarly and are simpler to handle since there is no $e^{r}$. When $j+|\alpha|+k+|\beta| \geq 2$, we iterate this process using Lemma 3.6. To complete the proof of the proposition, we finally note that (4-22) and (4-23) are easy consequences of (4-19) and Proposition 4.6.

By Propositions 4.7 and 4.8, we can define $r_{t}^{s}=r_{t}^{s}(r, \theta, \rho, \eta)$ and $\theta_{t}^{s}=\theta_{t}^{s}(r, \theta, \rho, \eta)$ on $\Gamma_{\mathrm{s}}^{ \pm}\left(\varepsilon_{1}\right)$ by

$$
r_{t}^{s}=r^{s}\left(r, \theta, \rho_{t}, \eta_{t}\right), \quad \theta_{t}^{s}=\theta^{s}\left(r, \theta, \rho_{t}, \eta_{t}\right) \quad \text { for } \pm t \geq \pm s \geq 0,
$$

where $\pm t \geq \pm s \geq 0$ means more precisely that $t \geq s \geq 0$ if $(r, \theta, \rho, \eta) \in \Gamma_{\mathrm{s}}^{+}\left(\varepsilon_{1}\right)$ and $t \leq s \leq 0$ if $(r, \theta, \rho, \eta) \in \Gamma_{\mathrm{s}}^{-}\left(\varepsilon_{1}\right)$. Here we assume that $\varepsilon_{1}$ is small enough so that Proposition 3.8 hold for $r>R\left(\varepsilon_{1}\right)$ and $\sigma=\frac{1}{2}$ (for instance), which justifies that $r_{t}^{s}$ and $\theta_{t}^{s}$ are well defined (and that their derivatives can be estimated using Proposition 3.8).

By the classical Hamilton-Jacobi theory, the function $\Sigma_{ \pm}$defined by

$$
\Sigma_{ \pm}(t, r, \theta, \rho, \eta)=r_{t}^{t} \rho+\theta_{t}^{t} \cdot \eta-t \rho^{2}-t e^{-2 r_{t}^{t}} q\left(r_{t}^{t}, \theta_{t}^{t}, \eta\right)
$$

solve the following time-dependent eikonal equation, for $(r, \theta, \rho, \eta) \in \Gamma_{\mathrm{s}}^{ \pm}\left(\varepsilon_{1}\right)$ and $\pm t \geq 0$,

$$
\partial_{t} \Sigma_{ \pm}=p\left(r, \theta, \partial_{r} \Sigma_{ \pm}, \partial_{\theta} \Sigma_{ \pm}\right),\left.\quad \Sigma_{ \pm}\right|_{t=0}=r \rho+\theta \cdot \eta
$$

To put it in a more standard way, note that (4-25) is obtained by defining $\Sigma_{ \pm}$via

$$
\Sigma_{ \pm}\left(t, r, \theta, \rho^{t}, \eta^{t}\right)=r^{t} \rho^{t}+\theta^{t} \cdot \eta^{t}-t p\left(r^{t}, \theta^{t}, \rho^{t}, \eta^{t}\right)
$$

(This simple expression uses the fact that $p$ is homogeneous of degree 2 in $(\rho, \eta)$.) Now assume for a while that

$$
S_{ \pm}(r, \theta, \rho, \eta):=r \rho+\theta \cdot \eta+\int_{0}^{ \pm \infty} \partial_{t}\left(\Sigma_{ \pm}(t, r, \theta, \rho, \eta)-t \rho^{2}\right) d t
$$

is well defined on $\Gamma_{\mathrm{s}}^{ \pm}\left(\varepsilon_{1}\right)$. Then, at least formally,

$$
\partial_{r, \theta} S_{ \pm}(r, \theta, \rho, \eta)=\lim _{t \rightarrow \pm \infty} \partial_{r, \theta} \Sigma_{ \pm}(t, r, \theta, \rho, \eta)
$$

The latter only uses the fact that the term $t \rho^{2}$ inside the integral is independent of $r, \theta$. If we know in addition that

$$
\lim _{t \rightarrow \pm \infty} \partial_{\rho} \Sigma_{ \pm}(t, r, \theta, \rho, \eta)=+\infty
$$


then, using the fact that $\Sigma^{ \pm}$are generating functions of $\Phi^{t}$, that is,

$$
\Phi^{t}\left(r, \theta, \partial_{r} \Sigma_{ \pm}, \partial_{\theta} \Sigma_{ \pm}\right)=\left(\partial_{\rho} \Sigma_{ \pm}, \partial_{\eta} \Sigma_{ \pm}, \rho, \eta\right) \text { for } \pm t \geq 0
$$

we obtain, on $\Gamma_{\mathrm{s}}^{ \pm}\left(\varepsilon_{1}\right)$,

$$
p\left(r, \theta, \partial_{r} S_{ \pm}, \partial_{\theta} S_{ \pm}\right)=\lim _{t \rightarrow \pm \infty} p\left(\partial_{\rho} \Sigma_{ \pm}, \partial_{\eta} \Sigma_{ \pm}, \rho, \eta\right)=\rho^{2} .
$$

Proposition 4.9. There exist $0<\varepsilon_{2} \leq \varepsilon_{1}$ such that we can find $S_{ \pm}=S_{ \pm}(r, \theta, \rho, \eta)$, defined on $\Gamma_{\mathrm{s}}^{ \pm}\left(\varepsilon_{2}\right)$, real-valued, satisfying

$$
p\left(r, \theta, \partial_{r} S_{ \pm}, \partial_{\theta} S_{ \pm}\right)=\rho^{2} \quad \text { on } \Gamma_{\mathrm{s}}^{ \pm}\left(\varepsilon_{2}\right)
$$

and such that

$$
S_{ \pm}(r, \theta, \rho, \eta)=r \rho+\theta \cdot \eta+\varphi_{ \pm}(r, \theta, \rho, \eta),
$$

for some $\varphi_{ \pm} \in \mathscr{M}_{\mathrm{hyp}}\left(\Gamma_{\mathrm{s}}^{ \pm}\left(\varepsilon_{2}\right)\right)$ satisfying, when $(r, \theta, \rho, 0) \in \Gamma_{\mathrm{s}}^{ \pm}\left(\varepsilon_{2}\right)$,

$$
\varphi_{ \pm \mid \eta=0}=0, \quad e^{r} \partial_{\eta} \varphi_{ \pm \mid \eta=0}=0, \quad e^{2 r} \operatorname{hess}_{\eta}\left[\varphi_{ \pm}\right]_{\mid \eta=0}=\int_{0}^{ \pm \infty} e^{-4 t \rho} \operatorname{hess}_{\eta}[q](r+2 t \rho, \theta) d t .
$$

It is convenient to note that, by possibly decreasing $\varepsilon_{2}$ and by using Lemma 2.4, (4-13), and the first two equalities in (4-35), we can write

with $a_{\beta}^{ \pm} \in C_{b}^{\infty}\left(F_{\text {hyp }}\left(\Gamma^{ \pm}\left(\varepsilon_{2}\right)\right)\right)$.

$$
\varphi_{ \pm}(r, \theta, \rho, \eta)=\sum_{|\beta|=2} a_{\beta}^{ \pm}\left(r, \theta, \rho, e^{-r} \eta\right) e^{-2 r} \eta^{\beta}
$$

Proof. We consider only the outgoing case. To complete the proof of (4-33), we have to prove the missing details, namely the convergence of the integral in (4-28) (plus its derivability) and the limits (4-29) and (4-30). Defining $\left(\rho_{t}^{s}, \eta_{t}^{s}\right):=\left(\rho^{s}, \eta^{s}\right)\left(r, \theta, \rho_{t}, \eta_{t}\right)$, the equations of motion yield

$$
r_{t}^{t}=r+2 \int_{0}^{t} \rho_{t}^{s} d s=r+2 t \rho-2 \int_{0}^{t} \int_{s}^{t} e^{-2 r_{t}^{u}}\left(2 q\left(r_{t}^{u}, \theta_{t}^{u}, \eta_{t}^{u}\right)-\left(\partial_{r} q\right)\left(r_{t}^{u}, \theta_{t}^{u}, \eta_{t}^{u}\right)\right) d u d s .
$$

By Propositions 3.8 and 4.8, we have the following bounds on $\Gamma_{\mathrm{s}}^{+}\left(\varepsilon_{1}\right)$, for $s \geq 0$ and $t \geq 0$,

$$
\left|D_{j \alpha k \beta}^{\text {hyp }}\left(r_{t}^{s}-r\right)\right| \lesssim\langle s\rangle, \quad\left|D_{j \alpha k \beta}^{\text {hyp }}\left(\theta_{t}^{s}-\theta\right)\right| \lesssim e^{-r}, \quad\left|D_{j \alpha k \beta}^{\text {hyp }}\left(\eta_{t}^{s}-\eta\right)\right| \lesssim 1 .
$$

In addition, using Proposition 3.4 and (4-18), we have, for $s \geq 0$ and $t \geq 0$,

$$
r_{t}^{s} \geq r+2\left(1-\varepsilon^{6}\right) s p^{1 / 2}\left(r, \theta, \rho_{t}, \eta_{t}\right)-C \geq r+s / 4-C \quad \text { on } \Gamma_{\mathrm{s}}^{+}\left(\varepsilon^{3}\right),
$$

with $\varepsilon$ small enough such that, $p^{1 / 2}\left(r, \theta, \rho_{t}, \eta_{t}\right) \geq \frac{1}{4}$. Using (4-37), (4-38), (4-39), with $\varepsilon_{2}:=\varepsilon^{3} \leq \varepsilon_{1}$ small enough, and Lemma 3.6, we obtain the existence of a bounded family $\left(a_{t}\right)_{t \geq 0}$ in $\mathscr{B}_{\text {hyp }}\left(\Gamma_{\mathrm{s}}^{+}\left(\varepsilon_{2}\right)\right)$ such that

$$
r_{t}^{t}=r+2 t \rho+a_{t}(r, \theta, \rho, \eta) \text { for } t \geq 0 .
$$

One shows similarly that $\left(\theta_{t}^{t}-\theta\right) \cdot \eta=e^{r}\left(\theta_{t}^{t}-\theta\right) \cdot e^{-r} \eta$ is bounded in $\mathscr{P}_{\mathrm{hyp}}\left(\Gamma_{\mathrm{s}}^{+}\left(\varepsilon_{2}\right)\right)$ for $t \geq 0$, and hence that

$$
\Sigma_{+}-\left(r \rho+\theta \cdot \eta+t \rho^{2}\right) \text { is bounded in } \mathscr{B}_{\mathrm{hyp}}\left(\Gamma_{\mathrm{s}}^{+}\left(\varepsilon_{2}\right)\right) \text { for } t \geq 0
$$


which proves (4-30). Then, using (4-26) and (4-31), we note that

$$
\partial_{t} \Sigma_{+}-\rho^{2}=e^{-2 \partial_{\rho} \Sigma_{+}} q\left(\partial_{\rho} \Sigma_{+}, \partial_{\eta} \Sigma_{+}, \eta\right) .
$$

Therefore, using (4-39), (4-40), (4-41) and (4-42), we obtain the convergence of the integral in (4-28) and the limit (4-29) as well as the fact that $S_{+}(r, \theta, \rho, \eta)-r \rho-\theta \cdot \eta$ belongs to $\mathscr{B}_{\mathrm{hyp}}\left(\Gamma_{\mathrm{s}}^{+}\left(\varepsilon_{2}\right)\right)$. Finally, the formulas in (4-35) follow directly from (4-42) combined with (4-22) and (4-15).

Remark 1. By applying $\partial_{\eta}$ to (4-41) we see that there exists $C$ such that

$$
\left|\partial_{\eta} \Sigma_{+}(t, r, \theta, \rho, \eta)-\theta\right| \leq C e^{-r} \lesssim e^{-R\left(\varepsilon_{2}\right)} \quad \text { for all }(r, \theta, \rho, \eta) \in \Gamma_{\mathrm{s}}^{+}\left(\varepsilon_{2}\right) \text { and } t \geq 0 .
$$

This shows, in the spirit of Corollary 3.10, that the proof above depends only on the definition of $q(r, \theta, \eta)$ for $\theta$ in an arbitrarily small neighborhood of $\bar{V}_{0}$, provided $\varepsilon_{2}$ is small enough.

Remark 2. Using (3-34), (3-35) and (4-20), one sees that $S_{ \pm}$is actually well defined on the conical area given by

$$
r>R\left(\varepsilon_{2}\right), \quad \theta \in V_{\varepsilon_{2}}, \quad \pm \rho>\left(1-\varepsilon_{2}^{2}\right) p^{1 / 2},
$$

and that

$$
\Sigma_{ \pm}(t, r, \theta, \lambda \rho, \lambda \eta)=\lambda \Sigma_{ \pm}(\lambda t, r, \theta, \rho, \eta) \quad \text { if } \lambda>0 .
$$

Thus that $S_{ \pm}$is the restriction to $\Gamma_{\mathrm{s}}^{ \pm}\left(\varepsilon_{2}\right)$ of an homogeneous function of degree 1 with respect to $(\rho, \eta)$.

We conclude this part with a result useful for considering phases globally defined on $\mathbb{R}^{2 n}$ when we shall construct Fourier integral operators.

Proposition 4.10. For some small enough $\varepsilon_{3}>0$, there exists a family of functions $\left(S_{ \pm, \varepsilon}\right)_{0<\varepsilon \leq \varepsilon_{3}}$, globally defined on $\mathbb{R}^{2 n}$, such that

$$
\varphi_{ \pm, \varepsilon}(r, \theta, \rho, \eta):=S_{ \pm, \varepsilon}(r, \theta, \rho, \eta)-r \rho-\theta \cdot \eta
$$

coincides with $\varphi_{ \pm}$on $\Gamma_{\mathrm{s}}^{ \pm}(\varepsilon)$ and satisfies

$$
\begin{gathered}
\operatorname{supp}\left(\varphi_{ \pm, \varepsilon}\right) \subset \Gamma_{\mathrm{s}}^{ \pm}\left(\varepsilon^{1 / 2}\right), \quad \varphi_{ \pm, \varepsilon} \in \mathscr{M}_{\mathrm{hyp}}\left(\Gamma_{\mathrm{s}}^{ \pm}\left(\varepsilon^{1 / 2}\right)\right), \\
\left|\partial_{\rho, \eta} \otimes \partial_{r, \theta} \varphi_{ \pm, \varepsilon}(r, \theta, \rho, \eta)\right| \leq \frac{1}{2} \quad \text { for }(r, \theta, \rho, \eta) \in \mathbb{R}^{2 n}, 0<\varepsilon \leq \varepsilon_{3},
\end{gathered}
$$

with $|\cdot|$ a matrix norm.

In further applications, (4-44) will also be used under the equivalent form

$$
\left|\partial_{\rho, \eta} \otimes \partial_{r, \theta} S_{ \pm, \varepsilon}(r, \theta, \rho, \eta)-\operatorname{Id}_{n}\right| \leq \frac{1}{2} \quad \text { for }(r, \theta, \rho, \eta) \in \mathbb{R}^{2 n}, 0<\varepsilon \leq \varepsilon_{3} .
$$

Remark. Although this proposition allows one to assume that they are globally defined, the phases $S_{ \pm, \varepsilon}$ solve the Hamilton-Jacobi equations on $\Gamma_{\mathrm{s}}^{ \pm}\left(\varepsilon_{2}\right)$ only.

Proof. We use Proposition 4.4 and consider

$$
S_{ \pm, \varepsilon}(r, \theta, \rho, \eta):=r \rho+\theta \cdot \eta+\chi_{\varepsilon^{1 / 2} \rightarrow \varepsilon}(r, \theta, \rho, \eta) \varphi_{ \pm}(r, \theta, \rho, \eta),
$$

with $\varphi_{ \pm}$defined in Proposition 4.9. We have $S_{ \pm, \varepsilon}=S_{ \pm}$on $\Gamma_{\mathrm{s}}^{ \pm}(\varepsilon)$ and, using(4-9) and (4-36),

$$
\left|\partial_{\rho, \eta} \otimes \partial_{r, \theta} S_{ \pm, \varepsilon}(r, \theta, \rho, \eta)-\operatorname{Id}_{n}\right| \lesssim \varepsilon^{1 / 4} \text { on } \mathbb{R}^{2 n},
$$


since $e^{-r}|\eta| \lesssim \varepsilon^{1 / 2}$ on $\Gamma_{\mathrm{s}}^{+}\left(\varepsilon^{1 / 2}\right)$. This yields the result if $\varepsilon$ is small.

4D. Fourier integral operators on $\mathbb{R}^{n}$. In this subsection, we derive some basic properties of Fourier integral operators associated to the phases $S_{ \pm}$obtained in Proposition 4.9.

For simplicity, we introduce the shorter notation

$$
\mathscr{P}_{\mathrm{s}}^{ \pm}(\varepsilon):=\mathscr{B}_{\mathrm{hyp}}\left(\Gamma_{\mathrm{s}}^{ \pm}(\varepsilon)\right), \quad \mathscr{S}_{\mathrm{s}}^{ \pm}(\varepsilon):=\mathscr{Y}_{\mathrm{hyp}}^{ \pm}\left(\Gamma_{\mathrm{s}}^{ \pm}(\varepsilon)\right),
$$

where the classes $\mathscr{B}_{\mathrm{hyp}}$ and $\mathscr{S}_{\text {hyp }}$ were defined in Definition 2.2.

By Propositions 4.9 and 4.10 , for all $h \in(0,1]$, all $\varepsilon$ small enough and all $a^{ \pm} \in \mathscr{Y}_{\mathrm{s}}^{ \pm}(\varepsilon)$, we can define the operator

$$
J_{h}^{ \pm}\left(a^{ \pm}\right): \mathscr{Y}\left(\mathbb{R}^{n}\right) \rightarrow \mathscr{S}\left(\mathbb{R}^{n}\right),
$$

as the operator with Schwartz kernel

$$
K_{h}^{ \pm}\left(r, \theta, r^{\prime}, \theta^{\prime}\right)=(2 \pi h)^{-n} \int e^{\frac{i}{h}\left(S_{ \pm}(r, \theta, \rho, \eta)-r^{\prime} \rho-\theta^{\prime} \cdot \eta\right)} a^{ \pm}(r, \theta, \rho, \eta) d \rho d \eta .
$$

Since the symbol $a^{ \pm}$is supported in $\Gamma_{\mathrm{s}}^{ \pm}(\varepsilon)$, the phase $S_{ \pm}$can be replaced by $S_{ \pm, \varepsilon}$ which is globally defined (see Proposition 4.10). Note also that $J_{h}^{ \pm}\left(a^{ \pm}\right)$maps clearly the Schwartz space into itself since, for fixed $h$ say $h=1$, it can be considered as the pseudodifferential operator with symbol $e^{i \varphi_{ \pm}} a^{ \pm}=$ $e^{i \varphi_{ \pm, \varepsilon}} a^{ \pm}$which belongs to $C_{b}^{\infty}\left(\mathbb{R}^{2 n}\right)$.

To obtain the $L^{2}$ boundedness of such operators uniformly in $h \in(0,1]$ as well as the factorization Proposition 4.13 below, which are both consequences of the usual Kuranishi trick, we need a preliminary result.

Consider the maps $\left(\underline{\rho}_{ \pm, \varepsilon}, \underline{\eta_{ \pm, \varepsilon}}\right): \mathbb{R}^{3 n} \rightarrow \mathbb{R}^{n}$ defined by

$$
\left(\underline{\rho_{ \pm, \varepsilon}}, \underline{\eta_{ \pm, \varepsilon}}\right)\left(r, \theta, r^{\prime}, \theta^{\prime}, \rho, \eta\right):=\int_{0}^{1} \partial_{r, \theta} S_{ \pm, \varepsilon}\left(r^{\prime}+s\left(r-r^{\prime}\right), \theta^{\prime}+s\left(\theta-\theta^{\prime}\right), \rho, \eta\right) d s
$$

so that

$$
\left(r-r^{\prime}\right) \underline{\rho}_{ \pm, \varepsilon}+\left(\theta-\theta^{\prime}\right) \cdot \underline{\eta}_{ \pm, \varepsilon}=S_{ \pm, \varepsilon}(r, \theta, \rho, \eta)-S_{ \pm, \varepsilon}\left(r^{\prime}, \theta^{\prime}, \rho, \eta\right) .
$$

Lemma 4.11. For all $\left(r, \theta, r^{\prime}, \theta^{\prime}\right) \in \mathbb{R}^{2 n}$ and all $0<\varepsilon \leq \varepsilon_{3}$, the map $(\rho, \eta) \mapsto\left(\underline{\rho}_{ \pm, \varepsilon}, \underline{\eta}_{ \pm, \varepsilon}\right)$ is a diffeomorphism from $\mathbb{R}^{n}$ onto itself. Denoting by $\left(\bar{\rho}_{ \pm, \varepsilon}, \bar{\eta}_{ \pm, \varepsilon}\right)$ the corresponding inverse, we have, for all $0<\varepsilon \leq \varepsilon_{3}$,

$$
\left|\partial_{\eta}^{\beta} \partial_{r}^{j} \partial_{r^{\prime}}^{j^{\prime}} \partial_{\theta}^{\alpha} \partial_{\theta^{\prime}}^{\alpha^{\prime}} \partial_{\rho}^{k}\left(\left(\bar{\rho}_{ \pm, \varepsilon}, \bar{\eta}_{ \pm, \varepsilon}\right)-(\rho, \eta)\right)\right| \lesssim 1 \quad \text { on } \mathbb{R}^{3 n} .
$$

Furthermore, there exists $\varepsilon_{6}>0$ such that, for all $0<\varepsilon \leq \varepsilon_{6}$, we have

$$
\begin{gathered}
(r, \theta, \rho, \eta) \in \Gamma_{\mathrm{s}}^{ \pm}(\varepsilon) \Longrightarrow\left(r, \theta, \underline{\rho}_{ \pm, \varepsilon}, \underline{\eta}_{ \pm, \varepsilon}\right)_{\mid r=r^{\prime}, \theta=\theta^{\prime}} \in \Gamma_{\mathrm{s}}^{ \pm}\left(\varepsilon^{1 / 3}\right), \\
\left(r, \theta, \underline{\rho}_{ \pm, \varepsilon}, \underline{\eta}_{ \pm, \varepsilon}\right)_{\mid r=r^{\prime}, \theta=\theta^{\prime}} \in \Gamma_{\mathrm{s}}^{ \pm}\left(\varepsilon^{3}\right) \Longrightarrow(r, \theta, \rho, \eta) \in \Gamma_{\mathrm{s}}^{ \pm}(\varepsilon),
\end{gathered}
$$

and

$$
\left|\partial_{\eta}^{\beta} \partial_{r}^{j} \partial_{r^{\prime}}^{j^{\prime}} \partial_{\theta}^{\alpha} \partial_{\theta^{\prime}}^{\alpha^{\prime}} \partial_{\rho}^{k}\left(\left(\bar{\rho}_{ \pm, \varepsilon}, \bar{\eta}_{ \pm, \varepsilon}\right)-(\rho, \eta)\right)_{\mid r=r^{\prime}, \theta=\theta^{\prime}}\right| \lesssim e^{-|\beta| r} \quad \text { on } \Gamma_{\mathrm{s}}^{+}\left(\varepsilon^{3}\right)
$$


Proof. The estimate (4-45) implies directly that $(\rho, \eta) \mapsto\left(\underline{\rho}_{ \pm, \varepsilon}, \underline{\eta}_{ \pm, \varepsilon}\right)$ is a diffeomorphism for all $\left(r, \theta, r^{\prime}, \theta^{\prime}\right) \in \mathbb{R}^{2 n}$ and $0<\varepsilon \leq \varepsilon_{3}$. Evaluating (4-50) at $\left(r, \theta, r^{\prime}, \theta^{\prime}, \bar{\rho}_{ \pm, \varepsilon}, \bar{\eta}_{ \pm, \varepsilon}\right)$, namely

$$
(\rho, \eta)=\left(\underline{\rho}_{ \pm, \varepsilon}, \underline{\eta}_{ \pm, \varepsilon}\right)\left(r, \theta, r^{\prime}, \theta^{\prime}, \bar{\rho}_{ \pm, \varepsilon}, \bar{\rho}_{ \pm, \varepsilon}\right)
$$

yields

$$
(\rho, \eta)-\left(\bar{\rho}_{ \pm, \varepsilon}, \bar{\eta}_{ \pm, \varepsilon}\right)=\int_{0}^{1} \partial_{r, \theta} \varphi_{ \pm, \varepsilon}\left(r^{\prime}+s\left(r-r^{\prime}\right), \theta^{\prime}+s\left(\theta-\theta^{\prime}\right), \bar{\rho}_{ \pm, \varepsilon}, \bar{\eta}_{ \pm, \varepsilon}\right) d s .
$$

By (4-43) we have $\varphi_{ \pm, \varepsilon} \in C_{b}^{\infty}\left(\mathbb{R}^{2 n}\right)$, so $\left(\bar{\rho}_{ \pm, \varepsilon}, \bar{\eta}_{ \pm, \varepsilon}\right)-(\rho, \eta)$ is bounded, for fixed $\varepsilon$. For the derivatives, we apply $\partial_{\eta}^{\beta} \partial_{r}^{j} \partial_{r^{\prime}}^{j^{\prime}} \partial_{\theta}^{\alpha} \partial_{\theta^{\prime}}^{\alpha^{\prime}} \partial_{\rho}^{k}$ to the right-hand side of (4-57) and obtain (4-52) by induction, using Lemma 3.6.

To prove (4-53), we simply notice that $\varphi_{ \pm, \varepsilon}$ coincides with $\varphi_{ \pm}$on $\Gamma_{\mathrm{s}}^{ \pm}\left(\varepsilon^{3}\right)$ so that

$$
\left|(\rho, \eta)-\left(\underline{\rho} \pm, \varepsilon, \underline{\eta_{ \pm}, \varepsilon}\right)_{\mid r=r^{\prime}, \theta=\theta^{\prime}}\right|=\left|\partial_{r, \theta} \varphi_{ \pm}(r, \theta, \rho, \eta)\right| \lesssim \varepsilon^{2},
$$

using (2-56) and (4-36). The result follows from Proposition 4.3 and the fact that $\Gamma_{\mathrm{s}}^{ \pm}(C \varepsilon) \subset \Gamma_{\mathrm{s}}^{ \pm}\left(\varepsilon^{1 / 3}\right)$ for $\varepsilon$ small enough. To get (4-54), we use directly Proposition A.1 proving that $\Gamma_{\mathrm{s}}^{ \pm}\left(\varepsilon^{3}\right) \subset \Psi^{t}\left(\Gamma_{\mathrm{s}}^{ \pm}(\varepsilon)\right)$ with

$$
\Psi^{t}(r, \theta, \rho, \eta):=\left(r, \theta, \underline{\rho}_{ \pm, \varepsilon}, \underline{\eta}_{ \pm, \varepsilon}\right)_{\mid r=r^{\prime}, \theta=\theta^{\prime}}=\left(r, \theta, \partial_{r} S_{ \pm}(r, \theta, \rho, \eta), \partial_{\theta} S_{ \pm}(r, \theta, \rho, \eta)\right)
$$

which is actually independent of $t$ and $\varepsilon$.

By (4-52), (4-55) holds when $\beta=0$. Consider next the first-order derivatives when $|\beta|=1$ and the other multi-indices are 0 . Applying $\partial_{\eta}^{\beta}$ to (4-56) and evaluating at $r=r^{\prime}, \theta=\theta^{\prime}$, we get

$$
\left(\partial_{\rho, \eta}\left(\underline{\rho}_{ \pm, \varepsilon}, \underline{\eta}_{ \pm, \varepsilon}\right)\right) \partial_{\eta}^{\beta}\left(\left(\bar{\rho}_{ \pm, \varepsilon}, \bar{\eta}_{ \pm, \varepsilon}\right)-(\rho, \eta)\right)=\partial_{\eta}^{\beta} \partial_{r, \theta} \varphi_{ \pm}\left(r, \theta, \bar{\rho}_{ \pm, \varepsilon}, \bar{\eta}_{ \pm, \varepsilon}\right)
$$

where we have replaced $\varphi_{ \pm, \varepsilon}$ by $\varphi_{ \pm}$using (4-54). Since $\left(\partial_{\rho, \eta}\left(\rho_{ \pm, \varepsilon}, \underline{\eta}_{ \pm, \varepsilon}\right)\right)^{-1}$ is uniformly bounded and $e^{r \beta} \partial_{\eta}^{\beta} \partial_{r, \theta} \varphi_{ \pm}\left(r, \theta, \bar{\rho}_{ \pm, \varepsilon}, \bar{\eta}_{ \pm, \varepsilon}\right)$ is bounded, using (4-54) again, we get the result in this case. Higher-order derivatives are obtained similarly by induction, using Lemma 3.6.

Proposition 4.12. For all $0<\varepsilon \leq \varepsilon_{6}$ and all $a^{ \pm}, b^{ \pm} \in \mathscr{S}_{\mathrm{s}}^{ \pm}(\varepsilon)$, we have

$$
\left\|J_{h}^{ \pm}\left(a^{ \pm}\right) J_{h}^{ \pm}\left(b^{ \pm}\right)^{*}-\sum_{k \leq N} h^{k} c_{k}^{ \pm}\left(r, \theta, h D_{r}, h D_{\theta}\right)\right\|_{L^{2}\left(\mathbb{R}^{n}\right) \rightarrow L^{2}\left(\mathbb{R}^{n}\right)} \leq C h^{N+1} \quad \text { for } h \in(0,1],
$$

where the constant $C$ can be chosen uniformly with respect to $a^{ \pm}$and $b^{ \pm}$when they vary in bounded subsets of $\mathscr{Y}_{\mathrm{s}}^{ \pm}(\varepsilon)$ and where the symbols $c_{k}^{ \pm}$are given by

$$
c_{k}^{ \pm}=\sum_{j+|\alpha|=k} \frac{1}{j ! \alpha !} \partial_{r^{\prime}}^{j} \partial_{\theta^{\prime}}^{\alpha} D_{\rho}^{j} D_{\eta}^{\alpha}\left(a\left(r, \theta, \bar{\rho}_{ \pm, \varepsilon}, \bar{\eta}_{ \pm, \varepsilon}\right) \overline{b\left(r^{\prime}, \theta^{\prime}, \bar{\rho}_{ \pm, \varepsilon}, \bar{\eta}_{ \pm, \varepsilon}\right)} \operatorname{Jac}\left(\bar{\rho}_{ \pm, \varepsilon}, \bar{\eta}_{ \pm, \varepsilon}\right)\right)_{\mid r=r^{\prime}, \theta=\theta^{\prime}},
$$

with $\operatorname{Jac}\left(\bar{\rho}_{ \pm, \varepsilon}, \bar{\eta}_{ \pm, \varepsilon}\right)=\left|\operatorname{det}\left(\partial_{\rho, \eta}\left(\bar{\rho}_{ \pm, \varepsilon}, \bar{\eta}_{ \pm, \varepsilon}\right)\right)\right|$. In particular

$$
c_{k}^{ \pm} \in \mathscr{Y}_{\mathrm{s}}^{ \pm}\left(\varepsilon^{1 / 3}\right) .
$$


Proof. The Schwartz kernel of $J_{h}^{ \pm}\left(a^{ \pm}\right) J_{h}^{ \pm}\left(b^{ \pm}\right)^{*}$ takes the form

$$
(2 \pi h)^{-n} \int e^{\frac{i}{h}\left(S_{ \pm, \varepsilon}(r, \theta, \rho, \eta)-S_{ \pm, \varepsilon}\left(r^{\prime}, \theta^{\prime}, \rho, \eta\right)\right)} a(r, \theta, \rho, \eta) \overline{b\left(r^{\prime}, \theta^{\prime}, \rho, \eta\right)} d \rho d \eta
$$

and this can be rewritten using the Kuranishi trick, that is, (4-51) and Lemma 4.11, as

$$
(2 \pi h)^{-n} \int e^{\frac{i}{h}\left(\left(r-r^{\prime}\right) \rho+\left(\theta-\theta^{\prime}\right) \cdot \eta\right)} a\left(r, \theta, \bar{\rho}_{ \pm, \varepsilon}, \bar{\eta}_{ \pm, \varepsilon} \overline{b\left(r^{\prime}, \theta^{\prime}, \bar{\rho}_{ \pm, \varepsilon}, \bar{\eta}_{ \pm, \varepsilon}\right)} \operatorname{Jac}\left(\bar{\rho}_{ \pm, \varepsilon}, \bar{\eta}_{ \pm, \varepsilon}\right) d \rho d \eta .\right.
$$

By (4-52), the symbol in (4-61) belongs to $C_{b}^{\infty}\left(\mathbb{R}^{3 n}\right)$. Therefore, the standard $h$-pseudodifferential calculus implies that, with $c_{k}$ defined by (4-59), we obtain the $L^{2}$ bound (4-58) by the CalderónVaillancourt theorem. In addition, by (4-53) (applied with $\left.(\rho, \eta)=\left(\bar{\rho}_{ \pm, \varepsilon}, \bar{\eta}_{ \pm, \varepsilon}\right)_{\mid r=r^{\prime}, \theta=\theta^{\prime}}\right)$, we have $\operatorname{supp}\left(c_{k}^{ \pm}\right) \subset \Gamma_{\mathrm{s}}^{+}\left(\varepsilon^{1 / 3}\right)$. One then checks that $c_{k}^{ \pm} \in \mathscr{B}_{\mathrm{s}}^{ \pm}\left(\varepsilon^{1 / 3}\right)$, using (4-55).

We note in passing that this proposition shows that, for all $0<\varepsilon \leq \varepsilon_{6}$ and all $a^{ \pm} \in \mathscr{Y}_{\mathrm{s}}^{ \pm}(\varepsilon)$,

$$
\left\|J_{h}^{ \pm}\left(a^{ \pm}\right)\right\|_{L^{2}\left(\mathbb{R}^{n}\right) \rightarrow L^{2}\left(\mathbb{R}^{n}\right)} \leq C \quad \text { for } h \in(0,1] .
$$

More precisely, the constant $C$ can be chosen independently of $a^{ \pm}$if, for $\varepsilon$ fixed, $a^{ \pm}$vary in a bounded subset of $\varphi_{\mathrm{s}}^{ \pm}(\varepsilon)$.

Proposition 4.13. For all $0<\varepsilon \leq \varepsilon_{6}$, the following holds: if we are given $a_{0}^{ \pm}, \ldots, a_{N}^{ \pm} \in \mathscr{S}_{\mathrm{s}}^{ \pm}(\varepsilon)$ with

$$
a_{0}^{ \pm} \gtrsim 1 \quad \text { on } \Gamma_{\mathrm{s}}^{ \pm}\left(\varepsilon^{3}\right)
$$

then, for all $\chi_{\mathrm{s}}^{ \pm} \in \mathscr{Y}_{\mathrm{s}}^{ \pm}\left(\varepsilon^{9}\right)$, we can find $b_{0}^{ \pm}, \ldots, b_{N}^{ \pm} \in \mathscr{Y}_{\mathrm{s}}^{ \pm}\left(\varepsilon^{3}\right)$ such that, if we set

$$
a^{ \pm}(h)=a_{0}^{ \pm}+\cdots+h^{N} a_{N}^{ \pm}, \quad b^{ \pm}(h)=b_{0}^{ \pm}+\cdots+h^{N} b_{N}^{ \pm},
$$

we have

$$
\left\|J_{h}^{ \pm}\left(a^{ \pm}(h)\right) J_{h}^{ \pm}\left(b^{ \pm}(h)\right)^{*}-\chi_{\mathrm{s}}^{ \pm}\left(r, \theta, h D_{r}, h D_{\theta}\right)\right\|_{L^{2}\left(\mathbb{R}^{n}\right) \rightarrow L^{2}\left(\mathbb{R}^{n}\right)} \leq C h^{N+1} \quad \text { for } h \in(0,1] .
$$

Proof. By Proposition 4.12 and the notation therein, we only need to find $b_{0}^{ \pm}, \ldots, b_{N}^{ \pm}$such that

$$
c_{0}^{ \pm}=\chi_{\mathrm{s}}^{ \pm}, \quad c_{k}^{ \pm}=0 \quad \text { for } k=1, \ldots, N .
$$

Using Lemma 4.11 and (4-59), the first equation, $c_{0}^{ \pm}=\chi_{\mathrm{s}}^{ \pm}$, is solved explicitly by

$$
\overline{b_{0}^{ \pm}(r, \theta, \rho, \eta)}=\left(\chi_{\mathrm{s}}^{ \pm}\left(r, \theta, \underline{\rho}_{ \pm, \varepsilon}, \underline{\eta}_{ \pm, \varepsilon}\right) \operatorname{Jac}\left(\underline{\rho}_{ \pm, \varepsilon}, \underline{\eta}_{ \pm, \varepsilon}\right)\right)_{\mid r^{\prime}=r, \theta^{\prime}=\theta} \times \frac{1}{a_{0}^{ \pm}(r, \theta, \rho, \eta)},
$$

where $1 / a_{0}^{ \pm}$is well defined since $\chi_{\mathrm{s}}^{ \pm}\left(r, \theta, \underline{\rho}_{ \pm, \varepsilon}, \underline{\eta}_{ \pm, \varepsilon}\right)_{\mid r^{\prime}=r, \theta^{\prime}=\theta}$ is supported in $\Gamma_{\mathrm{s}}^{ \pm}\left(\varepsilon^{3}\right)$ by (4-54). Thus, $b_{0}^{ \pm}$is well defined, supported in $\Gamma_{\mathrm{s}}^{ \pm}\left(\varepsilon^{3}\right)$ and belongs to $\mathscr{B}_{\mathrm{s}}^{ \pm}\left(\varepsilon^{3}\right)$ by (4-50) and Proposition 4.9 (since $\left(\underline{\rho}_{ \pm, \varepsilon}, \underline{\eta}_{ \pm, \varepsilon}\right)_{\mid r^{\prime}=r, \theta^{\prime}=\theta}=\partial_{r, \theta} S_{ \pm}$in $\left.\Gamma_{\mathrm{s}}^{ \pm}\left(\varepsilon^{3}\right)\right)$. Furthermore, $b_{0}^{ \pm}\left(r, \theta, \bar{\rho}_{ \pm, \varepsilon}, \bar{\eta}_{ \pm, \varepsilon}\right)_{r^{\prime}=r, \theta^{\prime}=\theta}$ is supported in $\Gamma_{\mathrm{s}}^{ \pm}\left(\varepsilon^{9}\right)$. We then find the other symbols by induction for we have a triangular system of equations. More precisely, the $k$-th equation $c_{k} \equiv 0(k \geq 1)$, reads

$$
\left(\overline{b_{k}^{ \pm}\left(r, \theta, \bar{\rho}_{ \pm, \varepsilon}, \bar{\eta}_{ \pm, \varepsilon}\right)} a_{0}^{ \pm}\left(r, \theta, \bar{\rho}_{ \pm, \varepsilon}, \bar{\eta}_{ \pm, \varepsilon}\right) \operatorname{Jac}\left(\bar{\rho}_{ \pm, \varepsilon}, \bar{\eta}_{ \pm, \varepsilon}\right)\right)_{\mid r=r^{\prime}, \theta=\theta^{\prime}}=d_{k}^{ \pm}(r, \theta, \rho, \eta)
$$


where $d_{k}^{ \pm}$is a linear combination of symbols of the form

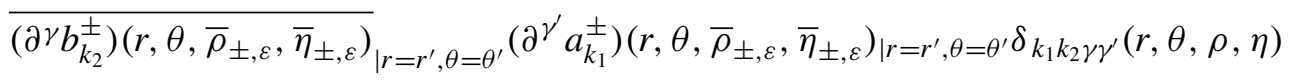

with $k_{2}<k$ and $\delta_{k_{1} k_{2} \gamma \gamma^{\prime}}$ a product of derivatives of order $\geq 1$ of $\left(\bar{\rho}_{ \pm, \varepsilon}, \bar{\eta}_{ \pm, \varepsilon}\right)\left(r, \theta, r^{\prime}, \eta^{\prime}, \rho, \eta\right)$ evaluated at $r=r^{\prime}, \theta=\theta^{\prime}$. By the induction assumption $\left(\partial^{\gamma} b_{k_{2}}^{ \pm}\right)\left(r, \theta, \bar{\rho}_{ \pm, \varepsilon}, \bar{\eta}_{ \pm, \varepsilon}\right)_{\mid r=r^{\prime}, \theta=\theta^{\prime}}$ is supported in $\Gamma_{\mathrm{s}}^{ \pm}\left(\varepsilon^{9}\right)$, so we have

$$
\left(r, \theta, \underline{\rho}_{ \pm, \varepsilon}, \underline{\eta}_{ \pm, \varepsilon}\right)_{\mid r=r^{\prime}, \theta=\theta^{\prime}} \in \Gamma_{\mathrm{s}}^{ \pm}\left(\varepsilon^{3}\right),
$$

using (4-53). Therefore, $\delta_{k_{1} k_{2} \gamma \gamma^{\prime}}\left(r, \theta, \underline{\rho}_{ \pm, \varepsilon}, \underline{\eta}_{ \pm, \varepsilon}\right)_{\mid r=r^{\prime}, \theta=\theta^{\prime}}$ belongs to $\mathscr{P}_{\mathrm{s}}^{ \pm}\left(\varepsilon^{3}\right)$ by (4-55) and $b_{k}^{ \pm}$satisfies the expected properties.

4E. The transport equations. In this subsection, we solve the time-independent transport equations related to the phases constructed in Proposition 4.9. If we define $\left(v^{ \pm}, w^{ \pm}\right)=\left(v^{ \pm}, w^{ \pm}\right)(r, \theta, \rho, \eta)$ by

$$
\left(\begin{array}{c}
v^{ \pm} \\
w^{ \pm}
\end{array}\right):=\left(\begin{array}{c}
\left(\partial_{\rho} p\right)\left(r, \theta, \partial_{r} S_{ \pm}, \partial_{\theta} S_{ \pm}\right) \\
\left(\partial_{\eta} p\right)\left(r, \theta, \partial_{r} S_{ \pm}, \partial_{\theta} S_{ \pm}\right)
\end{array}\right)=\left(\begin{array}{c}
2 \partial_{r} S_{ \pm} \\
e^{-2 r}\left(\partial_{\eta} q\right)\left(r, \theta, \partial_{\theta} S_{ \pm}\right)
\end{array}\right),
$$

these transport equations take the form

$$
v^{ \pm} \partial_{r} a^{ \pm}+w^{ \pm} \cdot \partial_{\theta} a^{ \pm}+y^{ \pm} a^{ \pm}=z^{ \pm},
$$

where $y^{ \pm}, z^{ \pm}$are given and $a^{ \pm}$is the unknown function of $(r, \theta, \rho, \eta)$. Such equations arise naturally in the construction of the Isozaki-Kiada parametrix (see Section 5). They can be solved standardly by the method of characteristics and therefore, we start with the study the integral curves of the vector field $\left(v^{ \pm}, w^{ \pm}\right)$.

Given $(r, \theta, \rho, \eta) \in \Gamma_{\mathrm{s}}^{ \pm}\left(\varepsilon^{2}\right)$, with $\varepsilon>0$ small enough (to be specified below), we denote by

$$
r_{t}^{ \pm}=r_{t}^{ \pm}(r, \theta, \rho, \eta), \quad \theta_{t}^{ \pm}=\theta_{t}^{ \pm}(r, \theta, \rho, \eta)
$$

the solution to

with initial data

$$
\left\{\begin{array}{l}
\dot{r}_{t}^{ \pm}=v^{ \pm}\left(r_{t}^{ \pm}, \theta_{t}^{ \pm}, \rho, \eta\right) \\
\dot{\theta}_{t}^{ \pm}=w^{ \pm}\left(r_{t}^{ \pm}, \theta_{t}^{ \pm}, \rho, \eta\right)
\end{array}\right.
$$

$$
r_{0}^{ \pm}(r, \theta, \rho, \eta)=r, \quad \theta_{0}^{ \pm}(r, \theta, \rho, \eta)=\theta .
$$

In this problem, $\rho$ and $\eta$ are parameters. Equivalently,

$$
\phi_{t}^{ \pm}=\phi_{t}^{ \pm}(r, \theta, \rho, \eta):=\left(r_{t}^{ \pm}, \theta_{t}^{ \pm}, \rho, \eta\right)
$$

is the flow of the autonomous vector field $\left(v^{ \pm}, w^{ \pm}, 0,0\right)$.

Proposition 4.14. There exists $\varepsilon_{4}>0$ such that for all $(r, \theta, \rho, \eta) \in \Gamma_{\mathrm{s}}^{ \pm}\left(\varepsilon_{4}^{2}\right)$, the solution $\left(r_{t}^{+}, \theta_{t}^{+}\right)$(resp. $\left.\left(r_{t}^{-}, \theta_{t}^{-}\right)\right)$is globally defined on $[0,+\infty)$ (resp. $\left.(-\infty, 0]\right)$. There also exists $C>0$ such that, for all $0<\varepsilon \leq \varepsilon_{4}$ and all $(r, \theta, \rho, \eta) \in \Gamma_{\mathrm{s}}^{ \pm}\left(\varepsilon^{2}\right)$, we have

$$
\left(r_{t}^{ \pm}, \theta_{t}^{ \pm}, \rho, \eta\right) \in \Gamma_{\mathrm{s}}^{ \pm}(\varepsilon) \quad \text { for } \pm t \geq 0
$$

and

$$
\left|r_{t}^{ \pm}-r-2 t \rho\right| \leq C \varepsilon^{2} \min (1,|t|), \quad\left|\theta_{t}^{ \pm}-\theta\right| \leq C e^{-r}
$$


Furthermore,

$$
\left|D_{\text {hyp }}^{j \alpha k \beta}\left(r_{t}^{ \pm}-r-2 t \rho\right)\right|+\left|D_{\text {hyp }}^{j \alpha k \beta}\left(\theta_{t}^{ \pm}-\theta\right)\right| \leq C_{j \alpha k \beta} .
$$

for $(r, \theta, \rho, \eta) \in \Gamma_{\mathrm{s}}^{ \pm}\left(\varepsilon_{4}^{2}\right)$ and $\pm t \geq 0$.

Since $S_{ \pm, \varepsilon}=S_{ \pm}$on $\Gamma_{\mathrm{s}}^{ \pm}(\varepsilon)$, the localization property (4-68) shows that $\phi_{t}^{ \pm}$still solves (4-66) on $\Gamma_{\mathrm{s}}^{ \pm}\left(\varepsilon^{2}\right)$ if one replaces $\left(v^{ \pm}, w^{ \pm}\right)$by $\left(v_{\varepsilon}^{ \pm}, w_{\varepsilon}^{ \pm}\right)$, the latter being obtained by replacing $S_{ \pm}$by $S_{ \pm, \varepsilon}$ in (4-64).

Proof. Here again we only consider the outgoing case. By (4-36), there exists $C_{0} \geq 1$ such that, for all $(r, \theta, \rho, \eta) \in \Gamma_{\mathrm{s}}^{+}\left(\varepsilon_{2}\right)$,

$$
\left|\partial_{r} S_{+}-\rho\right| \leq C_{0} e^{-r}|\eta| \quad \text { and } \quad\left|e^{-2 r}\left(\partial_{\eta} q\right)\left(r, \theta, \partial_{\theta} S_{+}\right)\right| \leq C_{0} e^{-2 r}|\eta| .
$$

By (2-56), there exists $C_{1} \geq 1$ such that, for all $\varepsilon>0$ small enough and all $(r, \theta, \rho, \eta) \in \Gamma_{\mathrm{s}}^{+}(\varepsilon)$, we have

$$
e^{-r}|\eta| \leq C_{1} \varepsilon \quad \text { and } \quad e^{-2 r}|\eta| \leq C_{1} \varepsilon^{2},
$$

the last inequality following from $e^{-R(\varepsilon)} \leq \varepsilon$. If $\varepsilon$ small enough, we may also assume that

$$
\rho>\frac{1}{8} \quad \text { for all }(r, \theta, \rho, \eta) \in \Gamma_{\mathrm{s}}^{+}(\varepsilon) .
$$

Now fix $M=5 C_{0} C_{1}$, and for $(r, \theta, \rho, \eta) \in \Gamma_{\mathrm{s}}^{+}\left(\varepsilon^{2}\right)$, consider $\mathcal{T}:=\mathscr{T}(r, \theta, \rho, \eta)$ defined by

$$
\mathscr{T}=\left\{t \geq 0:\left(r_{s}^{+}, \theta_{s}^{+}\right) \text {is defined and } r_{s}^{+} \geq r+s / 8,\left|\theta_{s}^{+}-\theta\right| \leq M \varepsilon^{2} \text { for all } s \in[0, t]\right\} .
$$

The set $\mathcal{T}$ is clearly an interval containing 0 and, if $\varepsilon$ is small enough, Proposition 4.3 shows that $\left(r_{s}^{+}, \theta_{s}^{+}, \rho, \eta\right) \in \Gamma_{\mathrm{s}}^{+}(\varepsilon)$ for all $s \in \mathcal{T}$. Thus, by (4-71) and (4-72), we have

$$
\left|\dot{r}_{s}^{+}-2 \rho\right| \leq 2 C_{0} C_{1} \varepsilon \quad \text { and } \quad\left|\dot{\theta}_{s}^{+}\right| \leq C_{0} C_{1} \varepsilon^{2} \quad \text { for } s \in \mathcal{T},
$$

and, by possibly assuming that $C_{0} C_{1} \varepsilon<\frac{1}{8}$, we have $\dot{r}_{s}^{+}>0$ on $\mathscr{T}$. Choosing $C_{M} \geq 1$ as in Proposition 4.3, we now claim that, if

$$
\varepsilon<\varepsilon_{2} / C_{M} \text { and } r>R\left(C_{M} \varepsilon\right),
$$

then $T:=\sup \mathscr{T}=+\infty$. Assume this is wrong. Then $T$ is finite, belongs to $\mathscr{T}$ and, on $[0, T]$, we have

$$
r_{s}^{+} \geq r+s / 8 \geq r, \quad\left|\theta_{s}^{+}-\theta\right| \leq C_{1} \varepsilon^{2}<M \varepsilon^{2},
$$

so, by Proposition 4.3, $\left(r_{s}^{+}, \theta_{s}^{+}, \rho, \eta\right) \in \Gamma_{\mathrm{s}}^{+}\left(C_{M} \varepsilon\right) \subset \Gamma_{\mathrm{s}}^{+}\left(\varepsilon_{2}\right)$ and, by (4-71) and (4-72),

$$
\begin{array}{r}
\left|r_{T}^{+}-r-2 \rho T\right| \leq C_{0} e^{-r}|\eta| \int_{0}^{T} e^{-s / 8} d s \leq C_{0} e^{-r}|\eta| T \leq C_{0} C_{1} \varepsilon T, \\
\left|\theta_{T}^{+}-\theta\right| \leq C_{0} e^{-2 r}|\eta| \int_{0}^{T} e^{-s / 4} d s \leq 4 C_{0} e^{-2 r}|\eta|<5 C_{0} C_{1} \varepsilon^{2} .
\end{array}
$$

This implies that $r_{T}^{+}>r+T / 8$ and that $\left|\theta_{T}^{+}-\theta\right|<M \varepsilon^{2}$, so the flow can be continued beyond $T$, yielding a contradiction with the definition of $T$. The flow is thus well defined for $t \geq 0$. Then (4-69) follows from the first inequalities of (4-73) and (4-74) with an arbitrary $t \geq 0$ instead of $T$, since $e^{-r}|\eta| \lesssim \varepsilon^{2}$ for $(r, \theta, \rho, \eta) \in \Gamma_{\mathrm{s}}^{+}\left(\varepsilon^{2}\right)$. If $\varepsilon$ is small enough, Proposition 4.3 shows that (4-68) is a direct consequence of (4-69), using that $e^{-r} \ll \varepsilon^{4}$. 
It remains to prove (4-70) for $j+|\alpha|+k+|\beta| \geq 1$. We consider $\bar{r}_{t}^{+}:=r_{t}^{+}-2 t \rho$ and $\bar{\theta}_{t}^{+}:=\theta_{t}^{+}$, which satisfy

with

$$
\frac{d \bar{r}_{t}^{+}}{d t}=\bar{v}\left(t, \bar{r}_{t}^{+}, \bar{\theta}_{t}^{+}, \rho, \eta\right), \quad \frac{d \bar{\theta}_{t}^{+}}{d t}=\bar{w}\left(t, \bar{r}_{t}^{+}, \bar{\theta}_{t}^{+}, \rho, \eta\right)
$$

$$
\begin{aligned}
& \bar{v}(t, r, \theta, \rho, \eta)=\left(\partial_{r} \varphi_{+}\right)(r+2 t \rho, \theta, \rho, \eta), \\
& \bar{w}(t, r, \theta, \rho, \eta)=e^{-2 r-4 t \rho}\left(\partial_{\eta} q\right)\left(r+2 t \rho, \theta, \partial_{\theta} S_{+}(r+2 t \rho, \theta, \rho, \eta)\right) .
\end{aligned}
$$

Using (4-36), we have, for all $j^{\prime}, \alpha^{\prime}, k^{\prime}, \beta^{\prime}$,

$$
\left|D_{\text {hyp }}^{j^{\prime} \alpha^{\prime} k^{\prime} \beta^{\prime}}(\bar{v}, \bar{w})\right| \lesssim\langle t\rangle^{k^{\prime}} e^{-4 t \rho} \lesssim e^{-2 t \rho} \quad \text { for } t \geq 0, \text { on } \Gamma_{\mathrm{s}}^{+}\left(\varepsilon_{2} / C\right),
$$

with $C$ such that if $(r, \theta, \rho, \eta) \in \Gamma_{\mathrm{s}}^{+}\left(\varepsilon_{2} / C\right)$ then $(r+2 t \rho, \theta, \rho, \eta) \in \Gamma_{\mathrm{s}}^{+}\left(\varepsilon_{2} / C\right)$. Note also that if $\varepsilon$ is small enough and $(r, \theta, \rho, \eta) \in \Gamma_{\mathrm{s}}^{+}\left(\varepsilon^{2}\right)$, we have $\left(\bar{r}_{t}^{+}, \bar{\theta}_{t}^{+}, \rho, \eta\right) \in \Gamma_{\mathrm{s}}^{+}\left(\varepsilon_{2} / C\right)$, using (4-69) and Proposition 4.3. We then obtain (4-70) by induction by applying $D_{\text {hyp }}^{j \alpha k \beta}$ to (4-75). Indeed, using Lemma 3.6 and (4-76), we have

$$
\frac{d}{d t} D_{\text {hyp }}^{j \alpha k \beta}\left(\bar{r}_{t}^{+}, \bar{\theta}_{t}^{+}\right)=\left(\partial_{r, \theta}(\bar{v}, \bar{w})\right) D_{\text {hyp }}^{j \alpha k \beta}\left(\bar{r}_{t}^{+}, \bar{\theta}_{t}^{+}\right)+\mathcal{O}\left(e^{-2 \rho t}\right),
$$

where $\mathcal{O}\left(e^{-2 \rho t}\right)=0$ for first-order derivatives and, otherwise, follows from the induction assumption. Since $\left|\partial_{r, \theta}(\bar{v}, \bar{w})\right| \lesssim e^{-2 \rho t}$, Lemma 3.7 yields the result.

We now come to the resolution of (4-65) in a way suitable to further purposes.

Proposition 4.15. There exists $\varepsilon_{5}>0$ such that, for all $0<\varepsilon \leq \varepsilon_{5}$ and all $y^{ \pm} \in \mathscr{B}_{\mathrm{hyp}}\left(\Gamma_{\mathrm{s}}^{ \pm}(\varepsilon)\right)$ of hyperbolic short range in $\Gamma_{\mathrm{s}}^{ \pm}(\varepsilon)$, the function

$$
a_{\mathrm{hom}}^{ \pm}=\exp \int_{0}^{ \pm \infty} y^{ \pm} \circ \phi_{s}^{ \pm} d s
$$

solves (4-65) on $\Gamma_{\mathrm{s}}^{ \pm}\left(\varepsilon^{2}\right)$ with $z^{ \pm} \equiv 0$, belongs to $\mathscr{B}_{\mathrm{hyp}}\left(\Gamma_{\mathrm{s}}^{ \pm}\left(\varepsilon^{2}\right)\right)$ and $a_{\mathrm{hom}}^{ \pm}-1$ is of hyperbolic long range in $\Gamma_{\mathrm{s}}^{ \pm}\left(\varepsilon^{2}\right)$.

In addition, for all $z^{ \pm} \in \mathscr{B}_{\mathrm{hyp}}\left(\Gamma_{\mathrm{s}}^{ \pm}(\varepsilon)\right)$, of hyperbolic short range in $\Gamma_{\mathrm{s}}^{ \pm}(\varepsilon)$, the function

$$
a_{\text {inhom }}^{ \pm}=-\int_{0}^{ \pm \infty} z^{ \pm} \circ \phi_{s}^{ \pm} \exp \left(\int_{0}^{s} y^{ \pm} \circ \phi_{u}^{ \pm} d u\right) d s
$$

solves (4-65) on $\Gamma_{\mathrm{s}}^{ \pm}\left(\varepsilon^{2}\right)$, belongs to $\mathscr{B}_{\mathrm{hyp}}\left(\Gamma_{\mathrm{s}}^{ \pm}\left(\varepsilon^{2}\right)\right)$, and is of hyperbolic long range in $\Gamma_{\mathrm{s}}^{ \pm}\left(\varepsilon^{2}\right)$.

Lemma 4.16. There exists $\varepsilon_{5}>0$ such that, for all $j, \alpha, k, \beta$ and all $N \geq 0$,

$$
\left|\partial_{r}^{j} \partial_{\theta}^{\alpha} \partial_{\rho}^{k} \partial_{\eta}^{\beta}\left(r_{t}^{ \pm}-r-2 t \rho\right)\right|+\left|\partial_{r}^{j} \partial_{\theta}^{\alpha} \partial_{\rho}^{k} \partial_{\eta}^{\beta}\left(\theta_{t}^{ \pm}-\theta\right)\right| \lesssim\langle r-\log \langle\eta\rangle\rangle^{-N},
$$

on $\Gamma_{\mathrm{s}}^{ \pm}\left(\varepsilon_{5}\right)$, uniformly with respect to $\pm t \geq 0$.

Proof. By Proposition 4.3, there exists $C>0$ such that, for all $\varepsilon$ small enough and all $s \in[0,1]$,

$$
(r, \theta, \rho, \eta) \in \Gamma_{\mathrm{s}}^{ \pm}\left(\varepsilon^{2}\right) \Longrightarrow(r, \theta, \rho, s \eta) \in \Gamma_{\mathrm{s}}^{ \pm}\left(C \varepsilon^{2}\right)
$$

Therefore, if $C \varepsilon^{2} \leq \varepsilon_{4}^{2}$ and if we set $X_{t}^{ \pm}(r, \theta, \rho, \eta)=\left(r_{t}^{ \pm}-r-2 t \rho, \theta_{t}^{ \pm}-\theta\right)$, we can write

$$
X_{t}^{ \pm}(r, \theta, \rho, \eta)=X_{t}^{ \pm}(r, \theta, \rho, 0)+\int_{0}^{1}\left(e^{r} \partial_{\eta} X_{t}^{ \pm}\right)(r, \theta, \rho, s \eta) d s \cdot e^{-r} \eta
$$


on $\Gamma_{\mathrm{s}}^{ \pm}\left(\varepsilon^{2}\right)$. The crucial remark is that $X_{t}^{ \pm}(r, \theta, \rho, 0)=0$. Indeed, by (4-34) and the first equation in (4-35), we have $\partial_{r} S_{ \pm} \equiv \rho$ and $\partial_{\theta} S_{ \pm} \equiv 0$ at $\eta=0$ (notice that $(r, \theta, \rho, 0) \in \Gamma_{\mathrm{s}}^{ \pm}\left(\varepsilon_{2}\right)$ if $C \varepsilon^{2} \leq \varepsilon_{2}$ ), so the solution to (4-66) is simply $(r+2 t \rho, \theta)$ in this case. In addition, by (4-70), $\left(X_{t}^{ \pm}\right)_{t \geq 0}$ is bounded in $\mathscr{B}_{\text {hyp }}\left(\Gamma_{\mathrm{s}}^{ \pm}\left(\varepsilon^{2}\right)\right)$. Thus, for all $N \geq 0$,

$$
\left|\partial_{r}^{j} \partial_{\theta}^{\alpha} \partial_{\rho}^{k} \partial_{\eta}^{\beta} X_{t}^{ \pm}(r, \theta, \rho, \eta)\right| \lesssim e^{-r}\langle\eta\rangle \lesssim\langle r-\log \langle\eta\rangle\rangle^{-N} \quad \text { for } \pm t \geq 0, \quad(r, \theta, \rho, \eta) \in \Gamma_{\mathrm{s}}^{ \pm}\left(\varepsilon^{2}\right),
$$

which yields the result.

Proof of Proposition 4.15. For simplicity we set $\partial^{\gamma}=\partial_{r}^{j} \partial_{\theta}^{\alpha} \partial_{\rho}^{k} \partial_{\eta}^{\beta}$. Then, using Lemma 3.6 with $|\gamma| \geq 1$, $\partial^{\gamma}\left(y^{ \pm} \circ \phi_{s}^{ \pm}\right)$is the sum of

$$
\left(\partial_{r} y^{ \pm}\right) \circ \phi_{s}^{ \pm} \partial^{\gamma} r_{s}^{ \pm}+\left(\partial_{\theta} y^{ \pm}\right) \circ \phi_{s}^{ \pm} \cdot \partial^{\gamma} \theta_{s}^{ \pm}+\delta_{j 0} \delta_{\alpha 0}\left(\partial_{\rho}^{k} \partial_{\eta}^{\beta} y^{ \pm}\right) \circ \phi_{s}^{ \pm}
$$

and of a linear combination of

$$
\left(\partial_{\rho}^{k-k^{\prime}} \partial_{\eta}^{\beta-\beta^{\prime}} \partial_{r, \theta}^{v} y^{ \pm}\right) \circ \phi_{s}^{ \pm}\left(\partial^{\gamma_{1}^{1}} r_{s}^{ \pm} \ldots \partial^{\gamma_{\nu_{1}}^{1}} r_{s}^{ \pm}\right) \ldots\left(\partial^{\gamma_{1}^{n}}\left(\theta_{s}^{ \pm}\right)_{n-1} \ldots \partial_{x}^{\gamma_{v_{n}}^{n}}\left(\theta_{s}^{ \pm}\right)_{n-1}\right),
$$

where $\left(\theta_{s}^{ \pm}\right)_{1}, \ldots,\left(\theta_{s}^{ \pm}\right)_{n-1}$ are the components of $\theta_{s}^{ \pm},\left(0,0, k^{\prime}, \beta^{\prime}\right)+\sum \gamma_{i}^{j}=\gamma$, using the convention and the notation of Lemma 3.6. By (4-70), we have

$$
\left|\left(\partial_{r} y^{ \pm}\right) \circ \phi_{s}^{ \pm} \partial^{\gamma} r_{s}^{ \pm}\right| \lesssim\left\langle r_{s}^{ \pm}-\log \langle\eta\rangle\right\rangle^{-\tau-2} e^{-r|\beta|}\langle s\rangle^{\kappa},
$$

where $\kappa=1$ if $k=1$ and $j+|\alpha|+|\beta|=0$, and $\kappa=0$ otherwise. On the other hand, by Lemma 4.16, we have

$$
\left|\left(\partial_{r} y^{ \pm}\right) \circ \phi_{s}^{ \pm} \partial^{\gamma} r_{s}^{ \pm}\right| \lesssim\left\langle r_{s}^{ \pm}-\log \langle\eta\rangle\right\rangle^{-\tau-2}\langle r-\log \langle\eta\rangle\rangle^{-\tilde{j}}\langle s\rangle^{\kappa},
$$

with the same $\kappa$ as above and $\tilde{j}=j$ if $j \geq 2$, or $\tilde{j}=0$ for $j \leq 1$. Similarly, we also have

$$
\left|\left(\partial_{\theta} y^{ \pm}\right) \circ \phi_{s}^{ \pm} \cdot \partial^{\gamma} \theta_{s}^{ \pm}\right| \lesssim\left\langle r_{s}^{ \pm}-\log \langle\eta\rangle\right\rangle^{-\tau-1} \min \left(e^{-|\beta| r},\langle r-\log \langle\eta\rangle\rangle^{-j}\right),
$$

while, for the last term of (4-78), we have

$$
\left|\delta_{j 0} \delta_{\alpha 0}\left(\partial_{\rho}^{k} \partial_{\eta}^{\beta} y^{ \pm}\right) \circ \phi_{s}^{ \pm}\right| \lesssim \min \left(e^{-|\beta| r} e^{-2|\beta \| \rho s|},\left\langle r_{s}^{ \pm}-\log \langle\eta\rangle\right\rangle^{-\tau-1-j}\right),
$$

since $e^{-|\beta| r_{s}^{ \pm}} \lesssim e^{-|\beta| r} e^{-2|\beta \| s|}$ for $r_{s}^{ \pm}-r-2 \rho s$ is bounded from below and $\rho s \geq 0$. Now, we remark that

$$
\left|\left(\partial^{\gamma_{1}^{1}} r_{s}^{ \pm} \ldots \partial^{\gamma_{v_{1}}^{1}} r_{s}^{ \pm}\right)\right| \lesssim\langle s\rangle^{\tilde{\nu}_{1}}\langle r-\log \langle\eta\rangle\rangle^{-N_{0}},
$$

where $\tilde{\nu}_{1}$ is the number of $\partial^{\gamma_{l}^{1}}=\partial_{r}^{j_{l}^{1}} \partial_{\theta}^{\alpha_{l}^{1}} \partial_{\rho}^{k_{l}^{1}} \partial_{\eta}^{\beta_{l}^{1}}$ for which $j_{l}^{1}=0, N_{0}=0$ if $j_{l}^{1} \leq 1$ for all $l$ and $N_{0}$ is any positive number if $j_{l}^{1} \geq 2$ for at least one $l$. We therefore obtain, if $\beta=\beta^{\prime}$,

$$
|(4-79)| \lesssim\left\langle r_{s}^{ \pm}-\log \langle\eta\rangle\right\rangle^{-\tau-1-\nu_{1}} \min \left(e^{-r|\beta|}\langle s\rangle^{\nu_{1}},\langle r-\log \langle\eta\rangle\rangle^{\nu_{1}-\tilde{\nu}_{1}-j}\langle s\rangle^{\tilde{\nu_{1}}}\right),
$$

since $v_{1}-\tilde{v}_{1}-j \geq 0$ in the case where no $r$ derivative fall on the components of $\theta_{s}^{ \pm}$and only $r$ derivatives of order at most 1 fall on $r_{s}^{ \pm}$. If $\beta \neq \beta^{\prime}$, we have

$$
|(4-79)| \lesssim \min \left(e^{-2\left|\beta-\beta^{\prime}\right||\rho s|} e^{-|\beta| r}\langle s\rangle^{\nu_{1}},\left\langle r_{s}^{ \pm}-\log \langle\eta\rangle\right\rangle^{-\tau-1-\nu_{1}}\langle r-\log \langle\eta\rangle\rangle^{\nu_{1}-\tilde{\nu}_{1}-j}\langle s\rangle^{\tilde{\nu}_{1}}\right) .
$$

Since $r_{s}^{ \pm}-r-2 \rho s$ is bounded from below, $\rho s \geq 0$ (with $|\rho| \gtrsim 1$ ) and using (4-17), we have

$$
\left\langle r_{s}^{ \pm}-\log \langle\eta\rangle\right\rangle^{-\tau-1-\nu_{1}} \lesssim\langle r-\log \langle\eta\rangle+|s|\rangle^{-\tau-1-\nu_{1}} .
$$


All this implies that

$$
\left|D_{\text {hyp }}^{j \alpha k \beta}\left(y^{ \pm} \circ \phi_{s}^{ \pm}\right)\right| \lesssim\langle s\rangle^{-\tau-1} \quad \text { and } \quad\left|\partial_{r}^{j} \partial_{\theta}^{\alpha} \partial_{\rho}^{k} \partial_{\eta}^{\beta}\left(y^{ \pm} \circ \phi_{s}^{ \pm}\right)\right| \lesssim\langle r-\log \langle\eta\rangle+|s|\rangle^{-\tau-1}\langle r-\log \langle\eta\rangle\rangle^{-j},
$$

and since

$$
\int_{0}^{+\infty}\langle r-\log \langle\eta\rangle+|s|\rangle^{-\tau-1} d s \lesssim\langle r-\log \langle\eta\rangle\rangle^{-\tau}
$$

(using (4-17) on strongly outgoing/incoming areas), we see that the function $\int_{0}^{ \pm \infty} y^{ \pm} \circ \phi_{s}^{ \pm} d s$ belongs to $\mathscr{B}_{\mathrm{hyp}}\left(\Gamma_{\mathrm{s}}^{ \pm}\left(\varepsilon^{2}\right)\right)$ and is of hyperbolic long range. This implies easily that the same holds for $a_{\mathrm{hom}}^{ \pm}-1$. One then checks that $a_{\mathrm{hom}}^{ \pm}$solves the homogeneous transport equation by computing $d\left(a_{\mathrm{hom}}^{ \pm} \circ \phi_{t}^{ \pm}\right) / d t$ at $t=0^{ \pm}$. One studies similarly the case of $a_{\text {inhom }}^{ \pm}$.

\section{An Isozaki-Kiada type parametrix}

In this section, we prove an approximation of $e^{-i t h P} \widehat{O p}_{\iota}\left(\chi_{\mathrm{s}}^{ \pm}\right)$when $\chi_{\mathrm{s}}^{ \pm}$is supported in the strongly outgoing $(+)$ or incoming $(-)$ region $\Gamma_{\iota, \mathrm{s}}^{ \pm}(\varepsilon)$ (see Definition 2.12 for these areas and Definition 2.1 for $\left.\widehat{O P}_{\iota}(\cdot)\right)$. We recall that $\iota$ is an arbitrary index corresponding to the chart at infinity we consider and where the symbols are supported (see (2-4) and (2-19)).

Here we will prove an $L^{2}$ approximation, valid for times such that $0 \leq \pm t \lesssim h^{-1}$. Basically, we will show that, for any $N, e^{-i t h P} \widehat{O P}_{\iota}\left(\chi_{\mathrm{s}}^{ \pm}\right)$is the sum of a Fourier integral operator and of a term of order $h^{N}$ in the operator norm of $L^{2}(M, \widehat{d G})$, uniformly for $0 \leq t \lesssim h^{-1}$.

We will therefore essentially prove half of Proposition 2.20, namely the estimate (2-79). The dispersion estimate (2-78), following from a stationary phase argument on the Fourier integral operator, will be proved in Section 7.

In the sequel, we choose an arbitrary $\iota \in \Phi$ (see (2-2)). Since it will be fixed, we drop it most of the time from the notation (in particular in phases, symbols) and keep it only for the diffeomorphism $\Psi_{\iota}$, the regions $\Gamma_{\imath, s}^{ \pm}(\cdot)$ and $(5-3)$.

In the next result, we use the classes of symbols $\mathscr{S}_{\text {hyp }}(\cdot)$ introduced in Definition 2.2 and the Fourier integral operators (4-49) defined in Section 4D. For these operators, the phases are associated to the Hamiltonian $p=p_{\iota}$, the principal symbol of $P$ in the $\iota$-th chart (this notation is consistant with (5-3)).

Theorem 5.1. For all $N \geq 0$, there exists $\varepsilon(N)>0$ such that, for all $0<\varepsilon \leq \varepsilon(N)$, the following holds: there exists $a^{ \pm}(h)=a_{0}^{ \pm}+\cdots+h^{N} a_{N}^{ \pm}$with $a_{0}^{ \pm}, \ldots, a_{N}^{ \pm} \in \mathscr{Y}_{\mathrm{hyp}}\left(\Gamma_{\iota, \mathrm{s}}^{ \pm}(\varepsilon)\right)$, such that for all

$$
\chi_{\mathrm{s}}^{ \pm} \in \mathscr{Y}_{\mathrm{hyp}}\left(\Gamma_{\iota, \mathrm{s}}^{ \pm}\left(\varepsilon^{9}\right)\right)
$$

we can find $b^{ \pm}(h)=b_{0}^{ \pm}+\cdots+h^{N} b_{N}^{ \pm}$with

$$
b_{0}^{ \pm}, \ldots, b_{N}^{ \pm} \in \mathscr{Y}_{\text {hyp }}\left(\Gamma_{\iota, \mathrm{s}}^{ \pm}\left(\varepsilon^{3}\right)\right),
$$

such that, for all $T>0$, there exists $C>0$ such that

$$
\left\|e^{-i t h P} \widehat{O p}_{\iota}\left(\chi_{\mathrm{s}}^{ \pm}\right)-\Psi_{\iota}^{*}\left(J_{h}^{ \pm}\left(a^{ \pm}(h)\right) e^{-i t h D_{r}^{2}} J_{h}^{ \pm}\left(b^{ \pm}(h)\right)^{*}\right)\left(\Psi_{\iota}^{-1}\right)^{*}\right\|_{L^{2}(\widehat{d G}) \rightarrow L^{2}(\widehat{d G})} \leq C h^{N-1},
$$

provided that

$$
0 \leq \pm t \leq T h^{-1}, \quad h \in(0,1]
$$


By the inclusions in (2-20), together with (2-21) and (2-54), the symbols $a^{ \pm}(h)$ and $b^{ \pm}(h)$ are supported in $\left(\varepsilon^{-1},+\infty\right) \times V_{\iota, \varepsilon} \times \mathbb{R}^{n} \subset\left(R_{\mathscr{K}}+1,+\infty\right) \times V_{\iota}^{\prime} \times \mathbb{R}^{n}$, for $\varepsilon$ small. Therefore the Schwartz kernel of the operator $J_{h}^{ \pm}\left(a^{ \pm}(h)\right) e^{-i t h D_{r}^{2}} J_{h}^{ \pm}\left(b^{ \pm}(h)\right)^{*}$ is supported in $\left(\left(R_{\mathscr{K}}+1,+\infty\right) \times V_{\iota}^{\prime}\right)^{2}$ and hence

$$
\Psi_{\iota}^{*}\left(J_{h}^{ \pm}\left(a^{ \pm}(h)\right) e^{-i t h D_{r}^{2}} J_{h}^{ \pm}\left(b^{ \pm}(h)\right)^{*}\right)\left(\Psi_{\iota}^{-1}\right)^{*}
$$

is well defined on the whole manifold (by the implicit requirement that its kernel vanishes outside the coordinate patch $u_{\iota} \times U_{\iota}$ of $\left.M \times M\right)$.

We also remark that $\varepsilon(N)$ could certainly be chosen independently of $N$. However this is useless for the applications we have in mind and we will not consider this refinement.

Before starting the proof, we give some heuristic ideas about our parametrix. It gives a microlocal approximation of $e^{i t h P}$ for initial data microlocalized in strongly outgoing/incoming areas. In such areas, $e^{-r} \eta$ is small and $r$ is large, so the geodesic flow is close to the "free" flow of $\rho^{2}$ uniformly in the future/past, as a consequence of Proposition 3.8 basically. This closeness at the classical level remains true at the quantum level in the sense that the flow $e^{i t h P}$ can be put in the normal form $e^{i t h D_{r}^{2}}$, i.e., up to the conjugation by time-independent Fourier integral operators. We point out that we state this approximation on a $h^{-1}$ time scale, but it would more generally hold for times of order $h^{-N}$, for any $N$. To obtain a semiglobal in time parametrix (one with $t \geq 0$ or $t \leq 0$ ), we would need to combine our construction with a priori estimates on $e^{i t h P}$ of local energy decay type, to control the error terms given by the Duhamel formula.

Let us fix or recall some notation. We set

$$
P_{\iota}=\left(\Psi_{\iota}^{-1}\right)^{*} P\left(\Psi_{\iota}\right)^{*}=p\left(r, \theta, D_{r}, D_{\theta}\right)+p_{1}\left(r, \theta, D_{r}, D_{\theta}\right)+p_{2}(r, \theta),
$$

with $p$ the principal symbol and $p_{k}$ of degree $2-k$ in $(\rho, \eta)$ for $k=1,2$. For simplicity, we also use the notation (4-48).

Recall finally that, for some fixed $\varepsilon_{\iota}>0$ small enough, Proposition 4.10 proves the existence of $S_{ \pm}$ solving

$$
p\left(r, \theta, \partial_{r} S_{ \pm}, \partial_{\theta} S_{ \pm}\right)=\rho^{2} \quad \text { for }(r, \theta, \rho, \eta) \in \Gamma_{\iota, \mathrm{s}}^{+}\left(\varepsilon_{\iota}\right) .
$$

Proof of Theorem 5.1. For simplicity we set

$$
A_{ \pm}=J_{h}^{ \pm}\left(a^{ \pm}(h)\right), \quad B_{ \pm}=J_{h}^{ \pm}\left(b^{ \pm}(h)\right) .
$$

By the Duhamel formula, we have

$$
e^{-i t h P} \Psi_{\iota}^{*} A_{ \pm}=\Psi_{\iota}^{*} A_{ \pm} e^{-i t h D_{r}^{2}}-\frac{i}{h} \int_{0}^{t} e^{-i(t-s) h P} \Psi_{\iota}^{*}\left(h^{2} P_{\iota} A_{ \pm}-A_{ \pm} h^{2} D_{r}^{2}\right) e^{-i s h D_{r}^{2}} d s .
$$

Multiplying (5-5) by $B_{ \pm}^{*}\left(\Psi_{\iota}^{-1}\right)^{*}$ and defining

$$
C_{ \pm}:=\chi_{\mathrm{s}}^{ \pm}\left(r, \theta, h D_{r}, h D_{\theta}\right)\left(\tilde{\kappa} \otimes \tilde{\kappa}_{\iota}\right)-A_{ \pm} B_{ \pm}^{*}, \quad D_{ \pm}(s):=\left(h^{2} P_{\iota} A_{ \pm}-A_{ \pm} h^{2} D_{r}^{2}\right) e^{-i s h D_{r}^{2}} B_{ \pm}^{*}
$$


(where $\tilde{\kappa}$ and $\tilde{\kappa}_{\iota}$ are the cutoffs used in Definition 2.1), we obtain $e^{-i t h P} \widehat{O P}_{\iota}\left(\chi_{\mathrm{s}}^{ \pm}\right)$

$$
=\Psi_{\iota}^{*} A_{ \pm} e^{-i t h D_{r}} B_{ \pm}^{*}\left(\Psi_{\iota}^{-1}\right)^{*}+e^{-i t h P} \Psi_{\iota}^{*} C_{ \pm}\left(\Psi_{\iota}^{-1}\right)^{*}-\frac{i}{h} \int_{0}^{t} e^{-i(t-s) h P} \Psi_{\iota}^{*} D_{ \pm}(s)\left(\Psi_{\iota}^{-1}\right)^{*} d s .
$$

Using (2-8) with $q=2$, the theorem will then be proved if we find $a^{ \pm}(h)$ and $b^{ \pm}(h)$ such that

$$
\left\|C_{ \pm}\right\|_{L^{2}\left(\mathbb{R}^{n}\right) \rightarrow L^{2}\left(\mathbb{R}^{n}\right)} \lesssim h^{N} \quad \text { and } \quad\left\|D_{ \pm}(s)\right\|_{L^{2}\left(\mathbb{R}^{n}\right) \rightarrow L^{2}\left(\mathbb{R}^{n}\right)} \lesssim h^{N+1} \quad \text { for } h \in(0,1],
$$

uniformly with respect to $0 \leq \pm s \leq T h^{-1}$ for $D_{ \pm}(s)$.

For simplicity we only consider the outgoing case but the incoming one is of course completely similar.

Construction of $\boldsymbol{a}^{+}(\boldsymbol{h})$. We first define $\left(v^{+}, w^{+}\right)$by (4-64) and also set

$$
y^{+}:=p\left(r, \theta, \partial_{r}, \partial_{\theta}\right) S_{+}+p_{1}\left(r, \theta, \partial_{r}, \partial_{\theta}\right) S_{+} .
$$

Lemma 5.2. There exists $\tilde{\varepsilon}_{\iota} \leq \varepsilon_{\iota}$ such that $y^{+}$belongs to $\mathscr{B}_{\mathrm{hyp}}\left(\Gamma_{\iota, \mathrm{s}}^{+}\left(\tilde{\varepsilon}_{\iota}\right)\right)$ and is of hyperbolic short range on $\Gamma_{\iota, \mathrm{s}}^{+}\left(\tilde{\varepsilon}_{\iota}\right)$.

Proof. This follows from (2-11) and (4-14), since Proposition 4.9 shows that $y_{\mid \eta=0}^{+} \equiv 0$.

Elementary computations show that, for all $a_{0}^{+}, \ldots, a_{N}^{+} \in \mathscr{Y}_{\text {hyp }}^{+}(\varepsilon)$ and $a^{+}(h)=a_{0}^{+}+\cdots+h^{N} a_{N}^{+}$,

$$
h^{2} P_{\iota} J_{h}^{+}\left(a^{+}(h)\right)-J_{h}^{+}\left(a^{+}(h)\right) h^{2} D_{r}^{2}=\sum_{l=0}^{N+2} h^{l} J_{h}^{+}\left(d_{l}^{+}\right),
$$

where the symbols are given by

$$
\begin{aligned}
d_{l}^{+} & =\left(p\left(r, \theta, \partial_{r} S_{+}, \partial_{\theta} S_{+}\right)-\rho^{2}\right) a_{l}^{+}-i\left(v^{+} \partial_{r} a_{l-1}^{+}+w^{+} \cdot \partial_{\theta} a_{l-1}^{+}+y^{+} a_{l-1}^{+}\right)+P_{\iota} a_{l-2}^{+} \\
& =-i\left(v^{+} \partial_{r} a_{l-1}^{+}+w^{+} \cdot \partial_{\theta} a_{l-1}^{+}+y^{+} a_{l-1}^{+}\right)+P_{\iota} a_{l-2}^{+},
\end{aligned}
$$

using (5-4) and assuming $\varepsilon \leq \varepsilon_{\imath}$. Here, we have $0 \leq l \leq N+2$ and the convention that $a_{-2}^{+}=a_{-1}^{+}=$ $a_{N+1}^{+}=a_{N+2}^{+} \equiv 0$. In particular, the first three terms are given by

$$
\begin{aligned}
d_{0}^{+} & =0, \\
i d_{1}^{+} & =v^{+} \partial_{r} a_{0}^{+}+w^{+} \cdot \partial_{\theta} a_{0}^{+}+y^{+} a_{0}^{+}, \\
i d_{2}^{+} & =v^{+} \partial_{r} a_{1}^{+}+w^{+} \cdot \partial_{\theta} a_{1}^{+}+y^{+} a_{1}^{+}+i P_{\iota} a_{0}^{+} .
\end{aligned}
$$

Using Proposition 4.15, Lemma 5.2 and assuming $\hat{\varepsilon}_{\iota} \leq \min \left(\tilde{\varepsilon}_{\iota}^{2}, \varepsilon_{5}\right)$ we can define

$$
\hat{a}_{0}^{+}(r, \theta, \rho, \eta)=\exp \int_{0}^{+\infty} y^{+} \circ \phi_{s}^{+}(r, \theta, \rho, \eta) d s \quad \text { for }(r, \theta, \rho, \eta) \in \Gamma_{\iota, \mathrm{s}}^{+}\left(\hat{\varepsilon}_{\iota}\right),
$$

so $\hat{a}_{0}^{+} \in \mathscr{B}_{\mathrm{hyp}}\left(\Gamma_{\iota, \mathrm{s}}^{+}\left(\hat{\varepsilon}_{\iota}\right)\right), \hat{a}_{0}^{+}-1$ is of hyperbolic long range in $\Gamma_{\iota, \mathrm{s}}^{+}\left(\hat{\varepsilon}_{\iota}\right)$ and

$$
v^{+} \partial_{r} \hat{a}_{0}^{+}+w^{+} \cdot \partial_{\theta} \hat{a}_{0}^{+}+y^{+} \hat{a}_{0}^{+} \equiv 0 \quad \text { on } \Gamma_{\iota, \mathrm{s}}^{+}\left(\hat{\varepsilon}_{\iota}\right) .
$$

Since the function $\int_{0}^{\infty} y^{+} \circ \phi_{s}^{+} d s$ is bounded on $\Gamma_{\iota, \mathrm{s}}^{+}\left(\hat{\varepsilon}_{\iota}\right)$ (see the proof of Proposition 4.15), we also have

$$
\hat{a}_{0}^{+}(r, \theta, \rho, \eta) \gtrsim 1 \quad \text { for }(r, \theta, \rho, \eta) \in \Gamma_{\iota, \mathrm{s}}^{+}\left(\hat{\varepsilon}_{\iota}\right) .
$$


Using (2-11) and the fact that $\hat{a}_{0}^{+}-1$ is of hyperbolic long range, it is easy to check that $P_{l} \hat{a}_{0}^{+}$is of hyperbolic short range in $\Gamma_{\iota, \mathrm{s}}^{+}\left(\hat{\varepsilon}_{\iota}^{2}\right)$. By Proposition 4.15, we can then define

$$
\hat{a}_{1}^{+}=i \int_{0}^{+\infty}\left(P_{\iota} \hat{a}_{0}^{+}\right) \circ \phi_{s}^{+} \exp \left(\int_{0}^{s} y^{+} \circ \phi_{u}^{+} d u\right) d s \quad \text { on } \Gamma_{\iota, \mathrm{s}}^{+}\left(\hat{\varepsilon}_{\iota}^{2}\right),
$$

which belongs to $\mathscr{B}_{\mathrm{hyp}}\left(\Gamma_{l, \mathrm{~s}}^{+}\left(\hat{\varepsilon}_{l}^{2}\right)\right)$, is of hyperbolic long range in $\Gamma_{l, \mathrm{~s}}^{+}\left(\hat{\varepsilon}_{l}\right)$ and satisfies

$$
v^{+} \partial_{r} \hat{a}_{1}^{+}+w^{+} \cdot \partial_{\theta} \hat{a}_{1}^{+}+y^{+} \hat{a}_{1}^{+} \equiv-i P_{\iota} \hat{a}_{0}^{+} \quad \text { on } \Gamma_{\iota, \mathrm{s}}^{+}\left(\hat{\varepsilon}_{\iota}^{2}\right) .
$$

More generally, for $1 \leq l \leq N$, we can define iteratively

$$
\hat{a}_{l}^{+}=i \int_{0}^{+\infty}\left(P_{\iota} \hat{a}_{l-1}^{+}\right) \circ \phi_{s}^{+} \exp \left(\int_{0}^{s} y^{+} \circ \phi_{u}^{+} d u\right) d s \quad \text { on } \Gamma_{\iota, \mathrm{s}}^{+}\left(\hat{\varepsilon}_{l}^{2^{l}}\right),
$$

which belongs to $\mathscr{B}_{\mathrm{hyp}}\left(\Gamma_{\iota, \mathrm{s}}^{+}\left(\hat{\varepsilon}_{l}^{2^{l}}\right)\right)$, is of hyperbolic long range in $\Gamma_{\iota, \mathrm{s}}^{+}\left(\hat{\varepsilon}_{l}^{2^{l}}\right)$ and satisfies

$$
v^{+} \partial_{r} \hat{a}_{l}^{+}+w^{+} \cdot \partial_{\theta} \hat{a}_{l}^{+}+y^{+} \hat{a}_{l}^{+} \equiv-i P_{\iota} \hat{a}_{l-1}^{+} \quad \text { on } \Gamma_{\iota, \mathrm{s}}^{+}\left(\hat{\varepsilon}_{l}^{2^{l}}\right),
$$

using Proposition 4.15 and the fact that $P_{l} \hat{a}_{l-1}^{+}$is of hyperbolic short range if $\hat{a}_{l}^{+}$is of hyperbolic long range. Therefore, using Proposition 4.4 with $\varepsilon \leq \hat{\varepsilon}_{l}^{2^{N}}$ and setting

$$
a_{l}^{+}=\chi_{\varepsilon^{2} \rightarrow \varepsilon}^{+} \hat{a}_{l}^{+} \quad \text { for } 0 \leq l \leq N,
$$

with the $\hat{a}_{l}^{+}$defined above, we have constructed $a_{0}^{+}, \ldots, a_{N}^{+} \in \mathscr{Y}_{\text {hyp }}^{+}(\varepsilon)$ with $a_{0}^{+}$satisfying (4-63), by (5-14). Furthermore,

$$
d_{l}^{+} \in \mathscr{Y}_{\text {hyp }}^{+}(\varepsilon) \text { for } 0 \leq l \leq N+2
$$

and

$$
d_{l}^{+} \equiv 0 \text { on } \Gamma_{l, \mathrm{~s}}^{+}\left(\varepsilon^{2}\right) \text { for } 0 \leq l \leq N .
$$

Construction of $\boldsymbol{b}^{+}(\boldsymbol{h})$. Given $\chi_{\mathrm{s}}^{+} \in \mathscr{S}_{\mathrm{hyp}}^{+}\left(\varepsilon^{9}\right)$, we simply choose the symbols $b_{0}^{+}, \ldots, b_{N}^{+}$according to Proposition 4.13, with $\varepsilon \leq \min \left(\hat{\varepsilon}_{l}^{2^{N}}, \varepsilon_{6}\right)$.

Justification of the parametrix. Since $\tilde{\kappa} \otimes \tilde{\kappa}_{\iota} \equiv 1$ near the support of $\chi_{\mathrm{s}}^{+}$, we have

$$
\left\|\chi_{\mathrm{s}}^{+}\left(r, \theta, h D_{r}, h D_{\theta}\right)-\chi_{\mathrm{s}}^{+}\left(r, \theta, h D_{r}, h D_{\theta}\right)\left(\tilde{\kappa} \otimes \tilde{\kappa}_{l}\right)\right\|_{L^{2}\left(\mathbb{R}^{n}\right) \rightarrow L^{2}\left(\mathbb{R}^{n}\right)} \lesssim h^{M}, \quad h \in(0,1],
$$

for all $M$, using the standard symbolic calculus, the Calderón-Vaillancourt Theorem and the fact that $\mathscr{S}_{\text {hyp }}^{+}(\varepsilon) \subset C_{b}^{\infty}\left(\mathbb{R}^{2 n}\right)$. Using Proposition 4.13, we therefore obtain

$$
\left\|C^{+}\right\|_{L^{2}\left(\mathbb{R}^{n}\right) \rightarrow L^{2}\left(\mathbb{R}^{n}\right)} \lesssim h^{N+1}, \quad h \in(0,1] .
$$

It remains to consider $D_{+}(s)$, which reads

$$
D_{+}(s)=\sum_{l=0}^{N+2} \sum_{m=0}^{N} h^{l+m} J_{h}^{+}\left(d_{l}^{+}\right) e^{-i s h D_{r}^{2}} J_{h}^{+}\left(b_{m}^{+}\right)^{*} .
$$

By (4-62) and (5-15), the part of the sum where $l \geq N+1$, has an $L^{2}$ operator norm of order $h^{N+1}$. Once divided by $h$ and integrated over an interval of size at most $h^{-1}$, the corresponding operator norm is $\mathcal{O}\left(h^{N-1}\right)$. The control of the other terms of the sum will follow from the next result. 
Proposition 5.3. If $\varepsilon$ is small enough, then, for all $0 \leq l, m \leq N$ and all $M \geq 0$, we have

$$
\left\|J_{h}^{+}\left(d_{l}^{+}\right) e^{-i s h D_{r}^{2}} J_{h}^{+}\left(b_{m}^{+}\right)^{*}\right\|_{L^{2}\left(\mathbb{R}^{n}\right) \rightarrow L^{2}\left(\mathbb{R}^{n}\right)} \leq C_{\varepsilon} h^{M} \quad \text { for } h \in(0,1], 0 \leq s \leq T h^{-1} .
$$

The proof is based on a fairly elementary nonstationary phase argument. To control the operator norms of the kernels obtained after integrations by parts, we need the following rough lemma.

Lemma 5.4. For $a \in C_{b}^{\infty}\left(\mathbb{R}^{3 n}\right)$ compactly supported with respect to $\rho$, let us set

$$
[a]_{h}^{+}\left(r, \theta, r^{\prime}, \theta^{\prime}\right)=(2 \pi h)^{-n} \iint e^{\frac{i}{h}\left(S_{+, \varepsilon}(r, \theta, \rho, \eta)-s \rho^{2}-S_{+, \varepsilon}\left(r^{\prime}, \theta^{\prime}, \rho, \eta\right)\right)} a\left(r, \theta, r^{\prime}, \theta^{\prime}, \rho, \eta\right) d \rho d \eta,
$$

using $S_{+, \varepsilon}$ defined in Proposition 4.10. Denote by $\mathscr{A}_{h}^{+}: L^{2}\left(\mathbb{R}^{n}\right) \rightarrow L^{2}\left(\mathbb{R}^{n}\right)$ the operator with Schwartz kernel $[a]_{h}^{+}$. Then, there exists $n_{0}(n) \geq 0$ such that, for all $\varepsilon$ small enough,

$$
\left\|\mathscr{A}_{h}^{+}\right\|_{L^{2}\left(\mathbb{R}^{n}\right) \rightarrow L^{2}\left(\mathbb{R}^{n}\right)} \leq C_{\varepsilon} h^{-n_{0}}\langle s\rangle^{n_{0}} \max _{|\gamma| \leq n_{0}} \sup _{\mathbb{R}^{3 n}}\left\|\partial^{\gamma} a\right\|_{\infty}
$$

for all $h \in(0,1]$, all $s \in \mathbb{R}$ and all $a \in C_{b}^{\infty}\left(\mathbb{R}^{3 n}\right)$ satisfying

$$
\operatorname{supp}(a) \subset\{|\rho| \leq 10\} \text {. }
$$

Proof. We get this as a simple consequence of the Calderón-Vaillancourt Theorem by interpreting $\mathscr{A}_{h}^{+}$ as the pseudodifferential operator with symbol

$$
e^{\frac{i}{h}\left(\varphi_{+, \varepsilon}(r, \theta, \rho, \eta)-s \rho^{2}-\varphi_{+, \varepsilon}\left(r^{\prime}, \theta^{\prime}, \rho, \eta\right)\right)} a\left(r, \theta, r^{\prime}, \theta^{\prime}, \rho, \eta\right),
$$

where $\varphi_{+, \varepsilon}$ is defined in Proposition 4.10.

Proof of Proposition 5.3. We notice first that, by Proposition 4.9 and (4-36),

$$
\begin{aligned}
& \partial_{\rho}\left(S_{+}(r, \theta, \rho, \eta)-s \rho^{2}-S_{+}\left(r^{\prime}, \theta^{\prime}, \rho, \eta\right)\right)=r-r^{\prime}-2 s \rho+\mathcal{O}\left(\varepsilon^{2}\right), \\
& \partial_{\eta}\left(S_{+}(r, \theta, \rho, \eta)-s \rho^{2}-S_{+}\left(r^{\prime}, \theta^{\prime}, \rho, \eta\right)\right)=\theta-\theta^{\prime}+\mathcal{O}\left(e^{-1 / \varepsilon}\right),
\end{aligned}
$$

on the support of $d_{l}^{+}(r, \theta, \rho, \eta) b_{m}^{+}\left(r^{\prime}, \theta^{\prime}, \rho, \eta\right)$. On the other hand, by construction, we have

$$
d_{l}^{+}=i^{-1}\left(v^{+} \partial_{r} \chi_{\varepsilon^{2} \rightarrow \varepsilon}+w^{+} \cdot \partial_{\theta} \chi_{\varepsilon^{2} \rightarrow \varepsilon}\right) \hat{a}_{l-1}^{+}+P_{l}\left(\chi_{\varepsilon^{2} \rightarrow \varepsilon} \hat{a}_{l-2}^{+}\right)-\chi_{\varepsilon^{2} \rightarrow \varepsilon} P_{l} \hat{a}_{l-2}^{+}
$$

(with the convention that $\hat{a}_{-2}^{+}=\hat{a}_{-1}^{+} \equiv 0$ ). Using in particular that

$$
w^{+}=e^{-r}\left(\partial_{\eta} q\right)\left(r, \theta, e^{-r} \partial_{\theta} S_{+}\right),
$$

we conclude that $d_{l}^{+}$is a sum of terms of the form $c(r, \theta, \rho, \eta) \partial_{r}^{j}\left(e^{-r} \partial_{\theta}\right)^{\alpha} \chi_{\varepsilon^{2} \rightarrow \varepsilon}^{+}$, with $j+|\alpha| \geq 1$ and $c \in \mathscr{B}_{\mathrm{s}}^{+}(\varepsilon)$. Using the form of $\chi_{\varepsilon^{2} \rightarrow \varepsilon}^{+}$given by Proposition 4.4, we see that, on the support of such terms, at least one of the following properties hold:

$$
\begin{aligned}
\varepsilon^{-1} \leq r \leq \varepsilon^{-2}, & \\
p(r, \theta, \rho, \eta) \leq \frac{1}{4}-\varepsilon^{2} \text { or } p(r, \theta, \rho, \eta) \geq 4 & +\varepsilon^{2}, \\
\varepsilon^{4-2 \kappa} \lesssim e^{-2 r}|\eta|^{2} & \lesssim \varepsilon^{2}, \\
\operatorname{dist}\left(\theta, V_{\iota}\right) & \geq \varepsilon^{4},
\end{aligned}
$$

for some fixed $0<\kappa<1$ in (5-21). For terms such that (5-19) holds on their supports, we have 


$$
(5-17) \leq \varepsilon^{-2}-\varepsilon^{-3}-2 s \rho+C \leq-1-2 s \rho .
$$

for $\varepsilon$ small enough and integrate by parts with respect to $\rho$. For those satisfying (5-20) on their supports, then we must have

$$
\rho^{2}-\frac{1}{4} \leq-\varepsilon^{2} \quad \text { or } \quad \rho^{2}-4 \gtrsim \varepsilon^{2},
$$

since $e^{-2 r}|\eta|^{2} \lesssim \varepsilon^{2}$ in any case, whereas on the support of $b_{l}^{+}$, where $p\left(r^{\prime}, \theta^{\prime}, \rho, \eta\right) \in\left(\frac{1}{4}-\varepsilon^{3}, 4+\varepsilon^{3}\right)$ and $e^{-2 r^{\prime}}|\eta|^{2} \lesssim \varepsilon^{6}$,

$$
\rho^{2}-\frac{1}{4} \gtrsim-\varepsilon^{3} \quad \text { and } \quad \rho^{2}-4 \leq \varepsilon^{3},
$$

so that the amplitude vanishes identically, again if $\varepsilon$ is small enough. For those satisfying (5-21) on their supports, we have $e^{r}|\eta|^{-1} \lesssim \varepsilon^{k-2}$. Since $e^{-r^{\prime}}|\eta| \lesssim \varepsilon^{3}$, we get

$$
e^{r-r^{\prime}} \leq C+(1+\kappa) \ln \varepsilon \ll 0,
$$

which implies again that $(5-17) \leq-1-2 s \rho$, if $\varepsilon$ is small enough. Thus on the supports of terms satisfying either (5-19) or (5-20) or (5-21), we have $|(5-17)| \gtrsim\langle s\rangle$. By standard integrations by parts, the kernel of corresponding operator can be written, for all $M$, as in Lemma 5.4 with amplitudes of order $(h /\langle s\rangle)^{M}$ in $C_{b}^{\infty}\left(\mathbb{R}^{3 n}\right)$. Hence, their $L^{2}$ operator norms are of order $(h /\langle s\rangle)^{M-n_{0}}$ with an arbitrary $M$.

For the remaining terms satisfying (5-22) on their supports, we remark that $\left|\theta^{\prime}-\theta\right| \geq \varepsilon^{5}$ (otherwise $\left.\operatorname{dist}\left(\theta, V_{\iota}\right) \leq\left|\theta-\theta^{\prime}\right|+\operatorname{dist}\left(\theta^{\prime}, V_{\iota}\right)<\varepsilon^{5}+\varepsilon^{6} \ll \varepsilon^{4}\right)$ hence

$$
|(5-18)| \gtrsim \varepsilon^{5} \text {. }
$$

Thus, for all $M \geq 0$, the kernel of the corresponding operators can be written as in Lemma 5.4 with amplitudes of order $h^{M}$ in $C_{b}^{\infty}\left(\mathbb{R}^{3 n}\right)$. Since $M$ is arbitrary, their $L^{2}$ operator norms are of order $h^{M}$ if $|s| \lesssim h^{-1}$.

This completes the proof of Theorem 5.1.

\section{Geometric optics and Egorov's theorem on AH manifolds}

As in the previous section, we fix here an arbitrary index $\iota$ corresponding a coordinate patch and then drop it from the notation in symbols, phases, intervals, etc.

6A. Finite time WKB approximation. Next we give a short time parametrix of $e^{-i t h P} \widehat{O p}{ }_{\iota}\left(\chi^{ \pm}\right)$when $\chi^{ \pm}$is supported in an outgoing (+) or an incoming (-) area. This parametrix is the standard geometric optic (or WKB) approximation which is basically well known. Nevertheless, in the literature, one mostly finds local versions (i.e., with $\chi \in C_{0}^{\infty}$ ) or versions in $\mathbb{R}^{n}$ for elliptic operators. Here we are neither in a relatively compact set nor in the uniformly elliptic setting, so we recall the construction with some details.

Analogously to Section 5, we prove here an $L^{2}$ approximation. The related dispersion estimates leading to (2-80) will be derived in Section 7.

We also emphasize that, although we shall prove this approximation with a specified time orientation ( $t \geq 0$ for $\chi^{+}$and $t \leq 0$ for $\chi^{-}$), this result has nothing to do with outgoing/incoming areas; in principle we should be able to state a similar result for any $\chi$ supported in $p^{-1}(I)$ and for times $|t| \ll 1$. We restrict the sense of time for only two reasons: firstly, because it is sufficient for our purpose and, secondly, 
because we can use directly Proposition 3.8 (we should otherwise give a similar result for the geodesic flow for $t$ in an open neighborhood of 0 ).

Fix

$$
I_{1} \Subset I_{2} \Subset I_{3} \Subset(0,+\infty),
$$

three relatively compact open subsets of $V_{\iota}^{\prime}$ (see (2-21)),

$$
V_{1} \Subset V_{2} \Subset V_{3} \Subset V_{\iota}^{\prime},
$$

and three real numbers

$$
-1<\sigma_{1}<\sigma_{2}<\sigma_{3}<1 .
$$

For some $R_{3}$ large enough to be fixed below, we also choose arbitrary $R_{1}, R_{2}$ real numbers such that

$$
R_{1}>R_{2}>R_{3}
$$

Theorem 6.1. For all $R_{3}$ large enough, there exists $t_{\mathrm{WKB}}>0$ and a function

$$
\Sigma \in C^{\infty}\left(\left[0, \pm t_{\mathrm{WKB}}\right] \times \mathbb{R}^{2 n}, \mathbb{R}\right)
$$

such that, for any

$$
\chi^{ \pm} \in \mathscr{S}_{\text {hyp }}\left(\Gamma_{\iota}^{ \pm}\left(R_{1}, V_{1}, I_{1}, \sigma_{1}\right)\right)
$$

we can find

$$
a_{0}^{ \pm}(t), \ldots, a_{N}^{ \pm}(t) \in \mathscr{Y}_{\text {hyp }}\left(\Gamma_{\iota}^{ \pm}\left(R_{2}, V_{2}, I_{2}, \sigma_{2}\right)\right),
$$

depending smoothly on $t$ for $0 \leq \pm t \leq t_{\mathrm{WKB}}$, and such that, if we set

$$
a_{N}^{ \pm}(t, h)=a_{0}^{ \pm}(t)+\cdots+h^{N} a_{N}^{ \pm}(t),
$$

the operator defined on $C_{0}^{\infty}\left(\mathbb{R}^{n}\right)$ by the kernel

$$
\left[\mathscr{F}_{h}^{ \pm}\left(t, a_{N}^{ \pm}(t, h)\right)\right]\left(t, r, \theta, r^{\prime}, \theta^{\prime}\right)=(2 \pi h)^{-n} \iint e^{\frac{i}{h}\left(\Sigma^{ \pm}(t, r, \theta, \rho, \eta)-r^{\prime} \rho-\theta^{\prime} \cdot \eta\right)} a_{N}^{ \pm}(t, h, r, \theta, \rho, \eta) d \rho d \eta,
$$

satisfies, with $\mathbf{1}_{\iota}$ the characteristic function of $\left(R_{3},+\infty\right) \times V_{3}$,

$$
\left\|e^{-i t h P} \widehat{O p}_{\iota}\left(\chi^{ \pm}\right)-\Psi_{\iota}^{*} \mathscr{\Psi}_{h}^{ \pm}\left(t, a_{N}^{ \pm}(t, h)\right) \mathbf{1}_{\iota}\left(\Psi_{\iota}^{-1}\right)^{*}\right\|_{L^{2}(\mathcal{M}, \widehat{d G}) \rightarrow L^{2}(\mathcal{M}, \widehat{d G})} \leq C h^{N+1},
$$

for

$$
0 \leq \pm t \leq t_{\mathrm{WKB}}, \quad h \in(0,1]
$$

In addition, the functions $\Sigma^{ \pm}$are of the form

$$
\Sigma^{ \pm}(t, r, \theta, \rho, \eta)=r \rho+\theta \cdot \eta+\left(\Sigma_{0}^{ \pm}(t, r, \theta, \rho, \eta)-r \rho-\eta \cdot \eta\right) \chi_{2 \rightarrow 3}^{ \pm}(r, \theta, \rho, \eta),
$$

with $\chi_{2 \rightarrow 3}^{ \pm} \in \mathscr{S}_{\text {hyp }}\left(\Gamma_{l}^{ \pm}\left(R_{3}, V_{3}, I_{3}, \sigma_{3}\right)\right)$ such that $\chi_{2 \rightarrow 3}^{ \pm} \equiv 1$ on $\Gamma_{l}^{ \pm}\left(R_{2}, V_{2}, I_{2}, \sigma_{2}\right)$, and some bounded family $\left(\Sigma_{0}^{ \pm}(t)\right)_{0 \leq \pm t \leq t_{\mathrm{WKB}}}$ in $\mathscr{S}_{\mathrm{hyp}}\left(\Gamma_{\iota}^{ \pm}\left(R_{3}, V_{3}, I_{3}, \sigma_{3}\right)\right)$ satisfying

$$
\left\{\begin{array}{l}
\partial_{t} \Sigma_{0}^{ \pm}+p\left(r, \theta, \partial_{r} \Sigma_{0}^{ \pm}, \partial_{\theta} \Sigma_{0}^{ \pm}\right)=0 \\
\Sigma_{0}^{ \pm}(0, r, \theta, \rho, \eta)=r \rho+\theta \cdot \eta
\end{array}\right.
$$

and

$$
\left|D_{\text {hyp }}^{j \alpha k \beta}\left(\Sigma_{0}^{ \pm}(t, r, \theta, \rho, \eta)-r \rho-\theta \cdot \eta-t p(r, \theta, \rho, \eta)\right)\right| \leq C_{j \alpha k \beta} t^{2}
$$


both for

$$
0 \leq \pm t \leq t_{\mathrm{WKB}} \quad \text { and } \quad(r, \theta, \rho, \eta) \in \Gamma_{\iota}^{ \pm}\left(R_{3}, V_{3}, I_{3}, \sigma_{3}\right)
$$

We also have

$$
\left(\Sigma^{ \pm}(t, r, \theta, \rho, \eta)-r \rho-\theta \cdot \eta\right)_{0 \leq \pm t \leq t_{\mathrm{WKB}}} \text { bounded in } \mathscr{S}_{\mathrm{hyp}}\left(\Gamma_{\iota}^{ \pm}\left(R_{3}, V_{3}, I_{3}, \sigma_{3}\right)\right) .
$$

Finally, for all $0 \leq j \leq N$,

$$
\left(a_{j}^{ \pm}(t)\right)_{0 \leq \pm t \leq t_{\mathrm{WKB}}} \text { is bounded in } \mathscr{Y}_{\mathrm{hyp}}\left(\Gamma_{\iota}^{ \pm}\left(R_{2}, V_{2}, I_{2}, \sigma_{2}\right)\right) .
$$

Notice that $V_{1} \Subset V_{\iota}^{\prime}$, so it makes sense to consider $\widehat{O p}_{\iota}\left(\chi^{ \pm}\right)$; see (2-23).

In principle it is not necessary to have $R_{3}$ large to get such a lemma, but this will be sufficient for our applications. The interest of choosing $R_{3}$ large is simply to allow to use directly Proposition 3.8.

Note also that, by (6-6), the kernel of $\mathscr{F}_{h}^{ \pm}\left(t, a_{N}^{ \pm}(t, h)\right) \mathbf{1}_{\iota}$ is supported in $\left(\left(R_{3},+\infty\right) \times V_{3}\right)^{2}$.

Proof of Theorem 6.1. The proof will occupy the rest of this section.

We need to find $\Sigma_{ \pm}$and $a_{N}^{ \pm}(t, h)$ such that

$$
\begin{aligned}
\mathscr{E}_{h}^{ \pm}\left(0, a_{N}^{ \pm}(0, h)\right) & =\chi^{ \pm}\left(r, \theta, h D_{r}, h D_{\theta}\right), \\
\left(h D_{t}+h^{2} P_{\iota}\right) \mathscr{F}_{h}^{ \pm}\left(t, a_{N}^{ \pm}(t, h)\right) & =h^{N+2} R_{N}^{ \pm}(t, h),
\end{aligned}
$$

where $P_{\iota}=\left(\Psi_{\iota}^{-1}\right)^{*} P \Psi_{\iota}^{*}$ and

$$
\left\|R_{N}^{ \pm}(t, h)\right\|_{L^{2}\left(\mathbb{R}^{n}\right) \rightarrow L^{2}\left(\mathbb{R}^{n}\right)} \leq C, \quad h \in(0,1], 0 \leq \pm t \leq t_{\mathrm{WKB}} .
$$

Indeed, if (6-7), (6-8) and (6-9) hold, the equality

$\Psi_{\iota}^{*} \mathscr{g}_{h}^{ \pm}\left(t, a_{N}^{ \pm}(t, h)\right) \mathbf{1}_{\iota}\left(\Psi_{\iota}^{-1}\right)^{*}-e^{-i t h P} \Psi_{\iota}^{*} \chi^{ \pm}\left(r, \theta, h D_{r}, h D_{\theta}\right) \mathbf{1}_{\iota}\left(\Psi_{\iota}^{-1}\right)^{*}$

will yield (6-2) since, for all $M>0$,

$$
=i h^{N+1} \int_{0}^{t} e^{-i(t-s) h \tilde{P}} \Psi_{\iota}^{*} R_{N}(s, h) \mathbf{1}_{\iota}\left(\Psi_{\iota}^{-1}\right)^{*} d s
$$

$$
\left\|\Psi_{\iota}^{*} \chi^{ \pm}\left(r, \theta, h D_{r}, h D_{\theta}\right) \mathbf{1}_{\iota}\left(\Psi_{\iota}^{-1}\right)^{*}-\widehat{O p}_{\iota}\left(\chi^{ \pm}\right)\right\|_{L^{2}(\mathcal{M}, \widehat{d G}) \rightarrow L^{2}(\mathcal{M}, \widehat{d G})} \leq C_{M} h^{M},
$$

by standard off-diagonal decay (see Definition 2.1 for $\left.\widehat{O P}_{\iota}\right)$, since $\mathbf{1}_{\iota} \equiv 1$ near $\Pi_{r, \theta}\left(\operatorname{supp}\left(\chi^{ \pm}\right)\right)$.

To get the conditions to be satisfied by $\Sigma^{ \pm}$and $a_{0}^{ \pm}, \ldots, a_{N}^{ \pm}$we observe that

$$
\left(h D_{t}+h^{2} P_{\iota}\right) \mathscr{F}_{h}^{ \pm}\left(t, a_{N}^{ \pm}(t, h)\right)=\sum_{j=0}^{N+2} h^{j} \mathscr{F}_{h}^{ \pm}\left(t, b_{j}^{ \pm}(t)\right),
$$

where, if we additionally set $a_{-2}^{ \pm}=a_{-1}^{ \pm}=a_{N+1}^{ \pm}=a_{N+2}^{ \pm} \equiv 0$,

$$
b_{j}=\left(\partial_{t} \Sigma^{ \pm}+p\left(r, \theta, \partial_{r} \Sigma^{ \pm}, \partial_{\theta} \Sigma^{ \pm}\right)\right) a_{j}^{ \pm}+i^{-1}\left(\partial_{t}+\mathscr{T}^{ \pm}\right) a_{j-1}^{ \pm}+P a_{j-2}^{ \pm},
$$

with

$$
\mathscr{T}^{ \pm}=2 \partial_{r} \Sigma^{ \pm} \partial_{r}+\left(\partial_{\eta} q\right)\left(r, \theta, e^{-r} \partial_{\theta} \Sigma^{ \pm}\right) \cdot e^{-r} \partial_{\theta}+\left(p+p_{1}\right)\left(r, \theta, \partial_{r}, \partial_{\theta}\right) \Sigma^{ \pm},
$$


where $q=q_{\iota}$ is defined in (2-13) and $p_{1}$ is the homogeneous part of degree 1 of the full symbol of $P_{l}$. To obtain (6-7), (6-8) and (6-9) it will therefore be sufficient to solve the eikonal equation (6-3), then the transport equations

$$
\begin{gathered}
\left(\partial_{t}+\mathscr{T}^{ \pm}\right) a_{0}^{ \pm}=0, \quad a_{0}^{ \pm}(0, \cdot)=\chi^{ \pm}(.), \\
\left(\partial_{t}+\mathscr{T}^{ \pm}\right) a_{k}^{ \pm}=-i P_{\iota} a_{k-1}^{ \pm}, \quad a_{k}^{ \pm}(0, \cdot)=0,
\end{gathered}
$$

for $1 \leq k \leq N$, and finally to get an $L^{2}$ bound for Fourier integral operators of the form $\mathscr{F}_{h}^{ \pm}(t, a)$ (using the Kuranishi trick).

To solve (6-3), we need the following lemma for which we recall that $\left(r^{t}, \theta^{t}, \rho^{t}, \eta^{t}\right)$ is the Hamiltonian flow of $p$.

Lemma 6.2. For all $-1<\sigma_{\text {eik }}<\sigma_{\text {eik }}^{\prime}<1$, all open intervals $I_{\text {eik }} \Subset I_{\text {eik }}^{\prime} \Subset(0,+\infty)$, all open subsets $V_{\text {eik }} \Subset V_{\text {eik }}^{\prime} \Subset V_{l}^{\prime}$ and all $R_{\text {eik }}>R_{\text {eik }}^{\prime}$ large enough, there exists $t_{1}>0$ small enough that

$$
\Psi_{ \pm}^{t}:(r, \theta, \rho, \eta) \mapsto\left(r^{t}, \theta^{t}, \rho, \eta\right)
$$

is a diffeomorphism from $\Gamma_{\iota}^{ \pm}\left(R_{\mathrm{eik}}^{\prime}, V_{\mathrm{eik}}^{\prime}, I_{\mathrm{eik}}^{\prime}, \sigma_{\mathrm{eik}}^{\prime}\right)$ onto its range for all $0 \leq \pm t<t_{1}$ and

$$
\Gamma_{\iota}^{ \pm}\left(R_{\mathrm{eik}}, V_{\mathrm{eik}}, I_{\mathrm{eik}}, \sigma_{\mathrm{eik}}\right) \subset \Psi_{ \pm}^{t}\left(\Gamma_{\iota}^{ \pm}\left(R_{\mathrm{eik}}^{\prime}, V_{\mathrm{eik}}^{\prime}, I_{\mathrm{eik}}^{\prime}, \sigma_{\mathrm{eik}}^{\prime}\right)\right) \quad \text { for all } 0 \leq \pm t<t_{1} .
$$

Proof. First choose a $\sigma_{\text {eik }}^{\prime \prime} \in \mathbb{R}$, and open interval $I_{\text {eik }}^{\prime \prime}$ and open set $V_{\text {eik }}^{\prime \prime}$ such that

$$
\sigma_{\text {eik }}^{\prime}<\sigma_{\text {eik }}^{\prime \prime}<1, \quad I_{\text {eik }}^{\prime} \Subset I_{\text {eik }}^{\prime \prime} \Subset(0,+\infty), \quad V_{\text {eik }}^{\prime} \Subset V_{\text {eik }}^{\prime \prime} \Subset V_{\iota}^{\prime} .
$$

Also choose $R_{\text {eik }}^{\prime \prime}>0$ large enough that Proposition 3.8 holds with $\sigma=\left|\sigma_{\text {eik }}^{\prime \prime}\right|$ and $R=R_{\text {eik }}^{\prime \prime}$. We then choose arbitrary $R_{\text {eik }}$ and $R_{\text {eik }}^{\prime}$ such that

$$
R_{\text {eik }}>R_{\text {eik }}^{\prime}>R_{\text {eik }}^{\prime \prime}
$$

and then $\chi_{\prime \rightarrow \prime \prime}^{ \pm} \in \mathscr{Y}_{\text {hyp }}\left(\Gamma_{\iota}^{ \pm}\left(R_{\text {eik }}^{\prime \prime}, V_{\text {eik }}^{\prime \prime}, I_{\text {eik }}^{\prime \prime}, \sigma_{\text {eik }}^{\prime \prime}\right)\right) \operatorname{such} \chi_{\prime \rightarrow \prime \prime}^{ \pm} \equiv 1$ on $\Gamma_{\iota}^{ \pm}\left(R_{\text {eik }}^{\prime}, V_{\text {eik }}^{\prime}, I_{\text {eik }}^{\prime}, \sigma_{\text {eik }}^{\prime}\right)$. The existence of such a function follows from Proposition 4.1(i). In particular, $\chi_{\prime \rightarrow \prime \prime}^{ \pm}$and $\partial_{r, \theta, \rho, \eta} \chi_{\prime \rightarrow \prime \prime}^{ \pm}$are bounded on $\mathbb{R}^{2 n}$. For $\pm t \geq 0$, consider the map

$$
\varepsilon_{ \pm}^{t}: \mathbb{R}^{2 n} \ni(r, \theta, \rho, \eta) \mapsto\left(\int_{0}^{t} 2 \rho^{s} d s, \int_{0}^{t} e^{-r^{s}}\left(\partial_{\eta} q\right)\left(r^{s}, \theta^{s}, e^{-r^{s}} \eta^{s}\right) d s\right) \chi_{\prime \rightarrow \prime \prime}^{ \pm}(r, \theta, \rho, \eta) \in \mathbb{R}^{n},
$$

so that, by the equations of motion,

$$
\Psi_{ \pm}^{t}=\operatorname{Id}_{\mathbb{R}^{2 n}}+\left(\varepsilon_{ \pm}^{t}, 0\right) \quad \text { on } \Gamma_{\iota}^{ \pm}\left(R_{\text {eik }}^{\prime}, V_{\text {eik }}^{\prime}, I_{\text {eik }}^{\prime}, \sigma_{\text {eik }}^{\prime}\right) .
$$

By Proposition 3.8 we have $\left|\partial_{r, \theta, \rho, \eta} \varepsilon_{ \pm}^{t}\right| \lesssim|t|$; hence $\operatorname{Id}_{\mathbb{R}^{2 n}}+\left(\varepsilon_{ \pm}^{t}, 0\right)$ is a diffeomorphism from $\mathbb{R}^{2 n}$ onto itself, for all $\pm t \geq 0$ small enough. Therefore, it remains to show that, if $t$ is small enough and $(r, \theta, \rho, \eta) \in \Gamma_{\iota}^{ \pm}\left(R_{\mathrm{eik}}, V_{\mathrm{eik}}, I_{\mathrm{eik}}, \sigma_{\mathrm{eik}}\right)$ is of the form

$$
(r, \theta, \rho, \eta)=\left(r^{\prime}, \theta^{\prime}, \rho^{\prime}, \eta^{\prime}\right)+\left(\varepsilon_{ \pm}^{t}\left(r^{\prime}, \theta^{\prime}, \rho^{\prime}, \eta^{\prime}\right), 0\right),
$$

then $\left(r^{\prime}, \theta^{\prime}, \rho^{\prime}, \eta^{\prime}\right) \in \Gamma_{\iota}^{ \pm}\left(R_{\text {eik }}^{\prime}, V_{\text {eik }}^{\prime}, I_{\text {eik }}^{\prime}, \sigma_{\text {eik }}^{\prime}\right)$. We have trivially $\rho=\rho^{\prime}$ and $\eta=\eta^{\prime}$. By Proposition 3.8, $\left|\varepsilon_{ \pm}^{t}\right| \lesssim|t|$ on $\mathbb{R}^{2 n}$, so $\left|r-r^{\prime}\right|+\left|\theta-\theta^{\prime}\right| \lesssim|t|$; hence $r^{\prime}>R_{\text {eik }}^{\prime}$ and $\theta^{\prime} \in V_{\text {eik }}^{\prime}$ if $t$ is small enough. Moreover, 
by writing

$$
q\left(r, \theta, e^{-r} \eta\right)-q\left(r^{\prime}, \theta^{\prime}, e^{-r^{\prime}} \eta\right)=q\left(r, \theta, e^{-r} \eta\right)-q\left(r^{\prime}, \theta^{\prime}, e^{-r} \eta\right)+\left(1-e^{-2\left(r^{\prime}-r\right)}\right) q\left(r^{\prime}, \theta^{\prime}, e^{-r} \eta\right)
$$

we see that

$$
\left|p(r, \theta, \rho, \eta)-p\left(r^{\prime}, \theta^{\prime}, \rho, \eta\right)\right| \lesssim|t|,
$$

using the boundedness of $\left|e^{-r^{\prime}} \eta\right|$ and Taylor's formula. Hence

$$
p\left(r^{\prime}, \theta^{\prime}, \rho, \eta\right) \in I_{\text {eik }}^{\prime} \quad \text { and } \quad \pm \rho>-\sigma_{\text {eik }}^{\prime} p\left(r^{\prime}, \theta^{\prime}, \rho, \eta\right)^{1 / 2}
$$

if $t$ is small enough, since $p(r, \theta, \rho, \eta) \in I_{\text {eik }}$ and $\pm \rho>-\sigma_{\text {eik }} p(r, \theta, \rho, \eta)^{1 / 2}$. This completes the proof.

Now fix $I_{\text {eik }}, I_{\text {eik }}^{\prime}, V_{\text {eik }}, V_{\text {eik }}^{\prime}$, and $\sigma_{\text {eik }}, \sigma_{\text {eik }}^{\prime}$ as in Lemma 6.2, with the additional conditions

$$
V_{\mathrm{eik}}=V_{3}, \quad I_{\mathrm{eik}}=I_{3}, \quad \sigma_{\mathrm{eik}}=\sigma_{3} .
$$

Denote by $\Psi_{t}^{ \pm}$the inverse of $\Psi_{ \pm}^{t}$ and define $\left(r_{t}, \theta_{t}\right)=\left(r_{t}, \theta_{t}\right)(r, \theta, \rho, \eta)$ by

$$
\Psi_{t}^{ \pm}(r, \theta, \rho, \eta)=\left(r_{t}, \theta_{t}, \rho, \eta\right) \in \Gamma_{\iota}^{ \pm}\left(R_{\mathrm{eik}}^{\prime}, V_{\mathrm{eik}}^{\prime}, I_{\mathrm{eik}}^{\prime}, \sigma_{\mathrm{eik}}^{\prime}\right),
$$

if $(r, \theta, \rho, \eta) \in \Gamma_{l}^{ \pm}\left(R_{\text {eik }}, V_{\text {eik }}, I_{\text {eik }}, \sigma_{\text {eik }}\right)$ and $0 \leq \pm t<t_{1}$. Here $t_{1}, R_{\text {eik }}$ and $R_{\text {eik }}^{\prime}$ are those given by Lemma 6.2.

Proposition 6.3. For all $R_{3}>R_{\mathrm{eik}}$, there exists $t_{\mathrm{eik}}>0$ such that

$$
\Sigma_{0}^{ \pm}(t, r, \theta, \rho, \eta):=r_{t} \rho+\theta_{t} \cdot \eta+t p\left(r_{t}, \theta_{t}, \rho, \eta\right),
$$

solves (6-3) on $\Gamma_{\iota}^{ \pm}\left(R_{3}, V_{3}, I_{3}, \sigma_{3}\right)$ for $0 \leq \pm t \leq t_{\mathrm{eik}}$, and such that

$$
\left(\Sigma_{0}^{ \pm}(t, r, \theta, \rho, \eta)-r \rho-\theta \cdot \eta\right)_{0 \leq \pm t \leq t_{\text {eik }}} \text { is bounded in } \mathscr{B}_{\mathrm{hyp}}\left(\Gamma_{\iota}^{ \pm}\left(R_{3}, V_{3}, I_{3}, \sigma_{3}\right)\right) .
$$

Proof. That $\Sigma_{0}^{ \pm}$solves the eikonal equation is standard, so we only have to show (6-16). Since

$$
\Sigma_{0}^{ \pm}(t, r, \theta, \rho, \eta)=r \rho+\theta \cdot \eta+\left(r_{t}-r\right) \rho+e^{r}\left(\theta_{t}-\theta\right) \cdot e^{-r} \eta+t e^{-2\left(r_{t}-r\right)} q\left(r_{t}, \theta_{t}, e^{-r} \eta\right),
$$

(6-16) would follow from the estimates

$$
\left|D_{\text {hyp }}^{j \alpha k \beta}\left(r_{t}-r\right)\right|+\left|D_{\text {hyp }}^{j \alpha k \beta}\left(e^{r}\left(\theta_{t}-\theta\right)\right)\right| \leq C_{j \alpha k \beta},
$$

for $0 \leq \pm t \leq \pm t_{\text {eik }}$ and $(r, \theta, \rho, \eta) \in \Gamma_{\iota}^{ \pm}\left(R_{3}, V_{3}, I_{3}, \sigma_{3}\right)$. The equations of motion yield

$$
r^{t}=r+\int_{0}^{t} 2 \rho^{s} d s, \quad \theta^{t}=\theta+\int_{0}^{t} e^{-r^{s}}\left(\partial_{\eta} q\right)\left(r^{s}, \theta^{s}, e^{-r^{s}} \eta^{s}\right) d s,
$$

so, by Proposition 3.8 with $R_{\text {eik }}^{\prime}$ of Lemma 6.2 and by choosing $t_{\text {eik }}$ small enough, we see that, for $0 \leq \pm t \leq t_{\mathrm{eik}}$

$$
\left|\partial_{r, \theta}\left(r^{t}, \theta^{t}\right)-\operatorname{Id}_{n}\right| \leq \frac{1}{2} \quad \text { on } \Gamma_{\iota}^{ \pm}\left(R_{\mathrm{eik}}^{\prime}, V_{\mathrm{eik}}^{\prime}, I_{\mathrm{eik}}^{\prime}, \sigma_{\mathrm{eik}}^{\prime}\right),
$$

where $|\cdot|$ is a matrix norm. Therefore, by differentiating the identity $\left(r^{t}, \theta^{t}\right)\left(r_{t}, \theta_{t}, \rho, \eta\right)=(r, \theta)$ one obtains, similarly to Proposition 4.8,

$$
\left|D_{\text {hyp }}^{j \alpha k \beta}\left(r_{t}-r\right)\right|+\left|D_{\text {hyp }}^{j \alpha k \beta}\left(\theta_{t}-\theta\right)\right| \leq C_{j \alpha k \beta},
$$


for $0 \leq \pm t \leq t_{\text {eik }}$ and $(r, \theta, \rho, \eta) \in \Gamma_{\iota}^{ \pm}\left(R_{3}, V_{3}, I_{3}, \sigma_{3}\right)$. This proves the expected estimates for $r_{t}-r$. The second equation of (6-18) evaluated at $\left(r_{t}, \theta_{t}, \rho, \eta\right)$ yields

$$
e^{r}\left(\theta-\theta_{t}\right)=\int_{0}^{t} e^{r-r_{t}^{s}}\left(\partial_{\eta} q\right)\left(r_{t}^{s}, \theta_{t}^{s}, e^{-r_{t}^{s}} \eta_{t}^{s}\right) d s,
$$

where $x_{t}^{s}=x^{s}\left(r_{t}, \theta_{t}, \rho, \eta\right)$ for $x=r, \theta, \eta$.

Combining (6-19) and Proposition 3.8, we have, on $\Gamma_{\iota}^{ \pm}\left(R_{3}, V_{3}, I_{3}, \sigma_{3}\right)$,

$$
\left|D_{\text {hyp }}^{j \alpha k \beta}\left(r_{t}^{s}-r\right)\right|+\left|D_{\text {hyp }}^{j \alpha k \beta}\left(\theta_{t}^{s}-\theta\right)\right|+\left|D_{\text {hyp }}^{j \alpha k \beta}\left(\eta_{t}^{s}-\eta\right)\right| \leq C_{j \alpha k \beta} \quad \text { for } 0 \leq \pm t, \pm s \leq t_{\text {eik }},
$$

from which the estimate of the second term of (6-17) follows using (6-20).

We now solve the transport equations. By (6-12), we have to consider the time-dependent vector field $\left(v_{t}^{ \pm}, w_{t}^{ \pm}\right)$defined on $\Gamma_{\iota}^{ \pm}\left(R_{3}, V_{3}, I_{3}, \sigma_{3}\right)$, for $0 \leq \pm t \leq t_{\mathrm{eik}}$, by

$$
\left(\begin{array}{c}
v_{t}^{ \pm} \\
w_{t}^{ \pm}
\end{array}\right):=\left(\begin{array}{c}
\left(\partial_{\rho} p\right)\left(r, \theta, \partial_{r} \Sigma_{0}^{ \pm}, \partial_{\theta} \Sigma_{0}^{ \pm}\right) \\
\left(\partial_{\eta} p\right)\left(r, \theta, \partial_{r} \Sigma_{0}^{ \pm}, \partial_{\theta} \Sigma_{0}^{ \pm}\right)
\end{array}\right)=\left(\begin{array}{c}
2 \partial_{r} \Sigma_{0}^{ \pm} \\
e^{-2 r}\left(\partial_{\eta} q\right)\left(r, \theta, \partial_{\theta} \Sigma_{0}^{ \pm}\right)
\end{array}\right) .
$$

We then denote by $\phi_{s \rightarrow t}^{ \pm}$the flow, from time $s$ to time $t$, of $\left(v_{t}^{ \pm}, w_{t}^{ \pm}, 0_{\mathbb{R}^{n}}\right)$ namely the solution to

$$
\partial_{t} \phi_{s \rightarrow t}^{ \pm}=\left(v_{t}^{ \pm}\left(\phi_{s \rightarrow t}^{ \pm}\right), w_{t}^{ \pm}\left(\phi_{s \rightarrow t}^{ \pm}\right), 0\right), \quad \phi_{s \rightarrow s}^{ \pm}(r, \theta, \rho, \eta)=(r, \theta, \rho, \eta) .
$$

Lemma 6.4. For any open interval $I_{\mathrm{tr}}$, any $\sigma_{\mathrm{tr}} \in \mathbb{R}$, and any open subset $V_{\mathrm{tr}} \subset \mathbb{R}^{n-1}$ such that

$$
R_{\text {tr }}>R_{3}, \quad V_{\text {tr }} \Subset V_{3}, \quad I_{\text {tr }} \Subset I_{3}, \quad-1<\sigma_{\text {tr }}<\sigma_{3},
$$

there exists $0<t_{2} \leq t_{\mathrm{eik}}$ small enough that

$$
\phi_{s \rightarrow t}^{ \pm} \text {is well defined on } \Gamma_{\iota}^{ \pm}\left(R_{\mathrm{tr}}, V_{\mathrm{tr}}, I_{\mathrm{tr}}, \sigma_{\mathrm{tr}}\right) \quad \text { for all } 0 \leq \pm s \leq t_{2}, 0 \leq \pm t \leq t_{2}
$$

and

$$
\left|D_{j \alpha k \beta}^{\mathrm{hyp}}\left(\phi_{s \rightarrow t}^{ \pm}-\mathrm{Id}\right)\right| \lesssim 1 \quad \text { on } \Gamma_{\iota}^{ \pm}\left(R_{\mathrm{tr}}, V_{\mathrm{tr}}, I_{\mathrm{tr}}, \sigma_{\mathrm{tr}}\right) \quad \text { for } 0 \leq \pm s, \pm t \leq t_{2} .
$$

By (6-23), we mean in particular that

$$
\phi_{s \rightarrow t}\left(\Gamma_{\iota}^{ \pm}\left(R_{\mathrm{tr}}, V_{\mathrm{tr}}, I_{\mathrm{tr}}, \sigma_{\mathrm{tr}}\right)\right) \subset \Gamma_{\iota}^{ \pm}\left(R_{3}, V_{3}, I_{3}, \sigma_{3}\right) \quad \text { for } 0 \leq \pm s, \pm t \leq t_{2} .
$$

The estimate (6-24) can be restated by saying that the components of $\phi_{s \rightarrow t}^{ \pm}-\mathrm{Id}$ are bounded families of $\mathscr{B}_{\mathrm{hyp}}\left(\Gamma_{\iota}^{ \pm}\left(R_{\mathrm{tr}}, V_{\mathrm{tr}}, I_{\mathrm{tr}}, \sigma_{\mathrm{tr}}\right)\right)$ for $0 \leq \pm s, \pm t \leq t_{2}$.

Proof. For all $\delta>0$ small enough, we have $\left|r-r^{\prime}\right|+\left|\theta-\theta^{\prime}\right| \leq \delta$ and $(r, \theta, \rho, \eta) \in \Gamma_{\iota}^{ \pm}\left(R_{\mathrm{tr}}, V_{\mathrm{tr}}, I_{\mathrm{tr}}, \sigma_{\mathrm{tr}}\right)$

$$
\Longrightarrow\left(r^{\prime}, \theta^{\prime}, \rho, \eta\right) \in \Gamma_{\iota}^{ \pm}\left(R_{3}, V_{3}, I_{3}, \sigma_{3}\right)
$$

by Proposition 4.2. Denoting by $\left(r_{s \rightarrow t}^{ \pm}, \theta_{s \rightarrow t}^{ \pm}, \rho, \eta\right)$ the components of $\phi_{s \rightarrow t}^{ \pm}$, they must be solutions of the problem

$$
\left(r_{s \rightarrow t}^{ \pm}, \theta_{s \rightarrow t}^{ \pm}\right)=(r, \theta)+\int_{s}^{t}\left(v_{\tau}^{ \pm}, w_{\tau}^{ \pm}\right)\left(r_{s \rightarrow \tau}^{ \pm}, \theta_{s \rightarrow \tau}^{ \pm}, \rho, \eta\right) d \tau .
$$

By (6-16), we have

$$
\left|\left(v_{\tau}^{ \pm}, w_{\tau}^{ \pm}\right)\right|+\left|\partial_{r, \theta}\left(v_{\tau}^{ \pm}, w_{\tau}^{ \pm}\right)\right| \leq C,
$$


on $\Gamma_{\iota}^{ \pm}\left(R_{3}, V_{3}, I_{3}, \sigma_{3}\right)$, for $0 \leq \pm \tau \leq t_{\text {eik }}$. Therefore, the sequence $u_{n}^{ \pm}(t)=u_{n}^{ \pm}(t, s, r, \theta, \rho, \eta)$ defined by

$$
u_{0}^{ \pm}(s)=(r, \theta), \quad u_{k+1}^{ \pm}(t)=(r, \theta)+\int_{s}^{t}\left(v_{\tau}^{ \pm}, w_{\tau}^{ \pm}\right)\left(u_{k}^{ \pm}(\tau), \rho, \eta\right) d \tau,
$$

is a Cauchy sequence in $C^{0}\left(\left[0, \pm t_{2}\right], \mathbb{R}^{n}\right)$ for all $(r, \theta, \rho, \eta) \in \Gamma_{\iota}^{ \pm}\left(R_{\mathrm{tr}}, V_{\mathrm{tr}}, I_{\mathrm{tr}}, \sigma_{\mathrm{tr}}\right)$ and $0 \leq \pm s \leq t_{2}$, for some $t_{2}$ small enough independent of $(r, \theta, \rho, \eta)$. Indeed, using (6-26) and choosing $t_{2}$ small enough so that $\sum_{k \geq 0}\left(C t_{2}\right)^{k+1} \leq \delta$, a standard induction using (6-27) shows that

$$
\left|u_{k+1}^{ \pm}(t)-u_{k}^{ \pm}(t)\right| \leq\left(C t_{2}\right)^{k+1},
$$

which makes the sequence well defined and convergent. This proves (6-23). We then obtain (6-24) by induction by differentiating the equations in (6-22). This proof is completely similar to that of the estimate (4-70) in Proposition 4.14 (and much simpler since it is local in time) so we omit the details.

Now denote by $q_{t}^{ \pm}=q_{t}^{ \pm}(r, \theta, \rho, \eta)$ the function defined on $\left[0, \pm t_{\text {eik }}\right] \times \Gamma_{\iota}^{ \pm}(R, V, I, \sigma)$ by

$$
q_{t}^{ \pm}:=\left(p+p_{1}\right)\left(r, \theta, \partial_{r}, \partial_{\theta}\right) \Sigma_{0}^{ \pm} .
$$

This function was involved in (6-12).

Proposition 6.5. Choose $R_{\mathrm{tr}}, V_{\mathrm{tr}}, I_{\mathrm{tr}}$ and $\sigma_{\mathrm{tr}}$ such that

$$
R_{2}>R_{\mathrm{tr}}>R_{3}, \quad V_{2} \Subset V_{\mathrm{tr}} \Subset V_{3}, \quad I_{2} \Subset I_{\mathrm{tr}} \Subset I_{3}, \quad \sigma_{2}<\sigma_{\mathrm{tr}}<\sigma_{3} .
$$

There exists $t_{\mathrm{tr}}>0$ small enough that, for all $\chi^{ \pm}$satisfying (6-1), the functions

$$
a_{0}^{ \pm}, \ldots, a_{N}^{ \pm}:\left[0, \pm t_{\text {tr }}\right] \times \mathbb{R}^{2 n} \rightarrow \mathbb{C}
$$

vanishing outside $\Gamma_{\iota}^{ \pm}\left(R_{2}, V_{2}, I_{2}, \sigma_{2}\right)$ and defined iteratively on $\Gamma_{\iota}^{ \pm}\left(R_{2}, V_{2}, I_{2}, \sigma_{2}\right)$ by

$$
\begin{aligned}
& a_{0}^{ \pm}(t):=\chi^{ \pm} \circ \phi_{t \rightarrow 0}^{ \pm} \exp \left(\int_{0}^{t} q_{s}^{ \pm} \circ \phi_{t \rightarrow s}^{ \pm}\right), \\
& a_{k}^{ \pm}(t):=-\int_{0}^{t} i\left(P_{\iota} a_{k-1}^{ \pm}\right)\left(s_{1}, \phi_{t \rightarrow s_{1}}^{ \pm}\right) \exp \left(\int_{s_{1}}^{t} q_{s_{2}}^{ \pm} \circ \phi_{t \rightarrow s_{2}}^{ \pm} d s_{2}\right) d s_{1} \quad \text { for } 1 \leq k \leq N
\end{aligned}
$$

are smooth and solve (6-13) and (6-14). Furthermore, for all $0 \leq k \leq N$,

$$
\left(a_{k}^{ \pm}(t)\right)_{0 \leq \pm t \leq t_{\text {tr }}} \text { is bounded in } \mathscr{Y}_{\text {hyp }}\left(\Gamma_{\iota}^{ \pm}\left(R_{2}, V_{2}, I_{2}, \sigma_{2}\right)\right) .
$$

Proof. Fix $R_{\mathrm{tr}}^{\prime}, V_{\mathrm{tr}}^{\prime}, I_{\mathrm{tr}}^{\prime}$ and $\sigma_{\mathrm{tr}}^{\prime}$ such that

$$
R_{2}>R_{\mathrm{tr}}^{\prime}>R_{\mathrm{tr}}, \quad V_{2} \Subset V_{\mathrm{tr}}^{\prime} \Subset V_{\mathrm{tr}}, \quad I_{2} \Subset I_{\mathrm{tr}}^{\prime} \Subset I_{\mathrm{tr}}, \quad \sigma_{2}<\sigma_{\mathrm{tr}}^{\prime}<\sigma_{\mathrm{tr}} .
$$

By choosing $0<t_{\mathrm{tr}} \leq t_{2}$ small enough, we then have, for all $0 \leq \pm s, \pm t \leq t_{\mathrm{tr}}$,

$$
\begin{gathered}
\phi_{s \rightarrow t}^{ \pm}\left(\Gamma_{\iota}^{ \pm}\left(R_{1}, V_{1}, I_{1}, \sigma_{1}\right)\right) \subset \Gamma_{\iota}^{ \pm}\left(R_{2}, V_{2}, I_{2}, \sigma_{2}\right), \\
\phi_{s \rightarrow t}^{ \pm}\left(\Gamma_{\iota}^{ \pm}\left(R_{2}, V_{2}, I_{2}, \sigma_{2}\right)\right) \subset \Gamma_{\iota}^{ \pm}\left(R_{\mathrm{tr}}^{\prime}, V_{\mathrm{tr}}^{\prime}, I_{\mathrm{tr}}^{\prime}, \sigma_{\mathrm{tr}}^{\prime}\right), \\
\phi_{s \rightarrow t}^{ \pm}\left(\Gamma_{\iota}^{ \pm}\left(R_{\mathrm{tr}}^{\prime}, V_{\mathrm{tr}}^{\prime}, I_{\mathrm{tr}}^{\prime}, \sigma_{\mathrm{tr}}^{\prime}\right)\right) \subset \Gamma_{\iota}^{ \pm}\left(R_{\mathrm{tr}}, V_{\mathrm{tr}}, I_{\mathrm{tr}}, \sigma_{\mathrm{tr}}\right) .
\end{gathered}
$$


This follows from Proposition 4.2 and the fact that $\left|\phi_{t \rightarrow s}^{ \pm}-\mathrm{Id}\right| \lesssim|t-s|$, which comes from the integration of (6-22) between $s$ and $t$, using (6-24). By Lemma 6.4, the flow is well defined on $\Gamma_{\iota}^{ \pm}\left(R_{\mathrm{tr}}, V_{\mathrm{tr}}, I_{\mathrm{tr}}, \sigma_{\mathrm{tr}}\right)$, therefore the condition (6-31) ensures that we have the pseudo-group property

$$
\phi_{t \rightarrow u}^{ \pm} \circ \phi_{s \rightarrow t}^{ \pm}=\phi_{s \rightarrow u}^{ \pm}, \quad 0 \leq \pm s, \pm t, \pm u \leq t_{\mathrm{tr}},
$$

on $\Gamma_{l}^{ \pm}\left(R_{\mathrm{tr}}^{\prime}, V_{\mathrm{tr}}^{\prime}, I_{\mathrm{tr}}^{\prime}, \sigma_{\mathrm{tr}}^{\prime}\right)$. In particular, $\phi_{t \rightarrow s}^{ \pm} \circ \phi_{s \rightarrow t}^{ \pm}=\mathrm{Id}$ on this set. Therefore, by (6-30), we have

$$
\Gamma_{\iota}^{ \pm}\left(R_{2}, V_{2}, I_{2}, \sigma_{2}\right) \subset \phi_{t \rightarrow s}^{ \pm}\left(\Gamma_{\iota}^{ \pm}\left(R_{\mathrm{tr}}^{\prime}, V_{\mathrm{tr}}^{\prime}, I_{\mathrm{tr}}^{\prime}, \sigma_{\mathrm{tr}}^{\prime}\right)\right) .
$$

This implies that the map

$$
(t, r, \theta, \rho, \eta) \mapsto\left(t, \phi_{s \rightarrow t}^{ \pm}(r, \theta, \rho, \eta)\right)
$$

is a diffeomorphism from $\left(0, \pm t_{\mathrm{tr}}\right) \times \Gamma_{l}^{ \pm}\left(R_{\mathrm{tr}}^{\prime}, V_{\mathrm{tr}}^{\prime}, I_{\mathrm{tr}}^{\prime}, \sigma_{\mathrm{tr}}^{\prime}\right)$ onto its range and that this range contains $\left(0, \pm t_{\mathrm{tr}}\right) \times \Gamma_{l}^{ \pm}\left(R_{2}, V_{2}, I_{2}, \sigma_{2}\right)$. Restricted to the latter set, the inverse is given by $\left(t, \phi_{t \rightarrow s}^{ \pm}\right)$which shows that $\phi_{t \rightarrow s}^{ \pm}$is smooth with respect to $t$. Furthermore, by differentiating in $t$ the relation $\phi_{t \rightarrow s}^{ \pm} \circ \phi_{s \rightarrow t}^{ \pm}=\mathrm{Id}$, one obtains

$$
\partial_{t} \phi_{t \rightarrow s}^{ \pm}+\left(\partial_{r, \theta} \phi_{t \rightarrow s}^{ \pm}\right) \cdot\left(v_{t}^{ \pm}, w_{t}^{ \pm}\right)=0, \quad \text { on } \Gamma_{\iota}^{ \pm}\left(R_{2}, V_{2}, I_{2}, \sigma_{2}\right),
$$

for $0< \pm t<t_{\mathrm{tr}}$. Using this relation, one easily checks that $a_{0}^{ \pm}$solves (6-13) on $\Gamma_{l}^{ \pm}\left(R_{2}, V_{2}, I_{2}, \sigma_{2}\right)$. In addition, if

$$
(r, \theta, \rho, \eta) \in \Gamma_{\iota}^{ \pm}\left(R_{\mathrm{tr}}^{\prime}, V_{\mathrm{tr}}^{\prime}, I_{\mathrm{tr}}^{\prime}, \sigma_{\mathrm{tr}}^{\prime}\right) \backslash \Gamma_{\iota}^{ \pm}\left(R_{2}, V_{2}, I_{2}, \sigma_{2}\right),
$$

we have $\phi_{t \rightarrow 0}^{ \pm}(r, \theta, \rho, \eta) \notin \operatorname{supp}\left(\chi^{ \pm}\right)$otherwise $(r, \theta, \rho, \eta) \in \Gamma_{l}^{ \pm}\left(R_{2}, V_{2}, I_{2}, \sigma_{2}\right)$ by (6-1), (6-29) and (6-32). This shows that, extended by 0 outside $\Gamma_{l}^{ \pm}\left(R_{2}, V_{2}, I_{2}, \sigma_{2}\right), a_{0}^{ \pm}$is smooth. The property (6-28) for $k=0$ is then a direct consequence of (6-24). We note in passing that we have

$$
\operatorname{supp}\left(a_{0}^{ \pm}(t)\right) \subset \phi_{0 \rightarrow t}^{ \pm}\left(\operatorname{supp}\left(\chi^{ \pm}\right)\right) .
$$

The proof for the higher-order terms $a_{k}^{ \pm}, k \geq 1$, is then obtained similarly by induction using that $\operatorname{supp}\left(P_{\iota} a_{k-1}^{ \pm}\left(s_{1}\right)\right) \subset \phi_{0 \rightarrow s_{1}}^{ \pm}\left(\operatorname{supp}\left(\chi^{ \pm}\right)\right)$for all $s_{1}$.

Proof of Theorem 6.1. There remains to prove (6-4), to globalize $\Sigma_{0}^{ \pm}$, to prove (6-5) and the bound (6-9). By Proposition 4.1, we can choose

$$
\chi_{2 \rightarrow 3}^{ \pm} \in \mathscr{S}_{\text {hyp }}\left(\Gamma_{\iota}^{ \pm}\left(R_{3}, V_{3}, I_{3}, \sigma_{3}\right)\right) \text { such that } \chi_{2 \rightarrow 3}^{ \pm} \equiv 1 \text { on } \Gamma_{\iota}^{ \pm}\left(R_{2}, V_{2}, I_{2}, \sigma_{2}\right) .
$$

We set

$$
\Sigma^{ \pm}(t, r, \theta, \rho, \eta)=r \rho+\theta \cdot \eta+\chi_{2 \rightarrow 3}^{ \pm}(r, \theta, \rho, \eta) \times\left(\Sigma_{0}^{ \pm}(t, r, \theta, \rho, \eta)-r \rho-\theta \cdot \eta\right) .
$$

It coincides with $\Sigma_{0}^{ \pm}$on $\left[0, \pm t_{\text {eik }}\right] \times \Gamma_{\iota}^{ \pm}\left(R_{2}, V_{2}, I_{2}, \sigma_{2}\right)$ so it is a solution to the eikonal equation on $\left[0, \pm t_{\mathrm{WKB}}\right] \times \Gamma_{\iota}^{ \pm}\left(R_{2}, V_{2}, I_{2}, \sigma_{2}\right)$, for any $0<t_{\mathrm{WKB}} \leq t_{\text {eik. }}$. Furthermore, (6-16) implies (6-5) and, by using

$$
\Sigma_{0}^{ \pm}(t, r, \theta, \rho, \eta)=r \rho+\theta \cdot \eta+\int_{0}^{t} p\left(r, \theta, \partial_{r} \Sigma_{0}^{ \pm}(s), \partial_{\theta} \Sigma_{0}^{ \pm}(s)\right) d s,
$$

we get (6-4) since (6-16) and (6-33) itself show that the components of $\left(\partial_{r} \Sigma^{ \pm}(s)-\rho, \partial_{\theta} \Sigma^{ \pm}(s)-\eta\right)$ are $\mathcal{O}(s)$ in $\mathscr{B}_{\mathrm{hyp}}\left(\Gamma_{\iota}^{ \pm}\left(R_{3}, V_{3}, I_{3}, \sigma_{3}\right)\right)$. 
To prove (6-9), we use the Kuranishi trick which is as follows. By Taylor's formula, we can write $\Sigma^{ \pm}(t, r, \theta, \rho, \eta)-\Sigma^{ \pm}\left(t, r^{\prime}, \theta^{\prime}, \rho, \eta\right)=\left(r-r^{\prime}\right) \tilde{\rho}^{ \pm}\left(t, r, \theta, r^{\prime}, \theta^{\prime}, \rho, \eta\right)+\left(\theta-\theta^{\prime}\right) \cdot \tilde{\eta}^{ \pm}\left(t, r, \theta, r^{\prime}, \theta^{\prime}, \rho, \eta\right)$. Using again (6-33) and (6-16), we obtain

$$
\left|\partial_{r}^{j} \partial_{\theta}^{\alpha} \partial_{r^{\prime}}^{j^{\prime}} \partial_{\theta^{\prime}}^{\alpha^{\prime}} \partial_{\rho}^{k} \partial_{\eta}^{\beta}\left(\left(\tilde{\rho}^{ \pm}, \tilde{\eta}^{ \pm}\right)\left(t, r, \theta, r^{\prime}, \theta^{\prime}, \rho, \eta\right)-(\rho, \eta)\right)\right| \leq C_{j \alpha j^{\prime} \alpha^{\prime} k \beta}|t|,
$$

for $\left(r, \theta, r^{\prime}, \theta^{\prime}, \rho, \eta \in \mathbb{R}^{3 n}\right)$ and $0 \leq \pm t \leq t_{\mathrm{eik}}$. The latter implies that, for all $0 \leq \pm t \leq t_{\mathrm{WKB}}$ small enough and all $\left(r, \theta, r^{\prime}, \theta^{\prime}\right) \in \mathbb{R}^{2 n}$, the map

$$
(\rho, \eta) \mapsto\left(\tilde{\rho}^{ \pm}, \tilde{\eta}^{ \pm}\right)
$$

is a diffeomorphism from $\mathbb{R}^{n}$ onto itself. Furthermore, proceeding similarly to the proof of (4-52) in Lemma 4.11, we see that its inverse $(\tilde{\rho}, \tilde{\eta}) \mapsto\left(\rho^{ \pm}, \eta^{ \pm}\right)$satisfies

$$
\left|\partial_{r}^{j} \partial_{\theta}^{\alpha} \partial_{r^{\prime}}^{j^{\prime}} \partial_{\theta^{\prime}}^{\alpha^{\prime}} \partial_{\tilde{\rho}}^{k} \partial_{\tilde{\eta}}^{\beta}\left(\left(\rho^{ \pm}, \eta^{ \pm}\right)\left(t, r, \theta, r^{\prime}, \theta^{\prime}, \tilde{\rho}, \tilde{\eta}\right)-(\tilde{\rho}, \tilde{\eta})\right)\right| \leq C_{j \alpha j^{\prime} \alpha^{\prime} k \beta},
$$

on $\mathbb{R}^{3 n}$, uniformly with respect to $0 \leq \pm t \leq t_{\mathrm{WKB}}$. Thus, for any bounded family $\left(a^{ \pm}(t)\right)_{0 \leq \pm t \leq t_{\mathrm{WKB}}}$ in $\mathscr{S}_{\text {hyp }}\left(\Gamma_{\iota}^{ \pm}\left(R_{2}, V_{2}, I_{2}, \sigma_{2}\right)\right)$, the kernel of $\mathscr{F}_{h}^{ \pm}\left(t, a^{ \pm}(t)\right) \mathscr{\Phi}_{h}^{ \pm}\left(t, a^{ \pm}(t)\right)^{*}$, which reads

$$
(2 \pi h)^{-n} \int e^{\frac{i}{h}\left(\Sigma^{ \pm}(t, r, \theta, \rho, \eta)-\Sigma^{ \pm}\left(t, r^{\prime}, \theta^{\prime}, \rho, \eta\right)\right)} a^{ \pm}(t, r, \theta, \rho, \eta) \overline{a^{ \pm}\left(t, r^{\prime}, \theta^{\prime}, \rho, \eta\right)} d \rho d \eta,
$$

can be written as

$$
(2 \pi h)^{-n} \int e^{\left.\frac{i}{h}\left(\left(r-r^{\prime}\right) \tilde{\rho}+\left(\theta-\theta^{\prime}\right) \cdot \tilde{\eta}\right)\right)} B\left(t, r, \theta, r^{\prime}, \theta^{\prime}, \tilde{\rho}, \tilde{\eta}\right) d \tilde{\rho} d \tilde{\eta},
$$

with $B(t, \cdot)$ bounded in $C_{b}^{\infty}\left(\mathbb{R}^{3 n}\right)$ as $0 \leq \pm t \leq t_{\mathrm{WKB}}$. By the Calderón-Vaillancourt theorem the operator given by (6-37) is uniformly bounded; hence $\left\|\mathscr{F}_{h}^{ \pm}\left(t, a^{ \pm}(t)\right)\right\|_{L^{2}\left(\mathbb{R}^{n}\right) \rightarrow L^{2}\left(\mathbb{R}^{n}\right)} \leq C$ whenever $0 \leq \pm t \leq t_{\mathrm{WKB}}$ and $h \in(0,1]$, where $C$ depends only a finite number of seminorms of $a^{ \pm}(t)$ in $C_{b}^{\infty}\left(\mathbb{R}^{2 n}\right)$. Using (6-10), (6-11) (with $a_{k}^{ \pm}(t)$ solutions to the transport equations) and (6-28), the bound above yields (6-9), which completes the proof of Theorem 6.1.

6B. Proof of Proposition 2.24. To prove Proposition 2.24, we first need a version of the semiclassical Egorov Theorem in the asymptotically hyperbolic setting. We recall that $\Phi^{t}=\left(r^{t}, \theta^{t}, \rho^{t}, \eta^{t}\right)$ denotes the Hamiltonian flow of the principal symbol $p$ of $P$.

Fix an open subset $V \Subset V_{\iota}^{\prime}$, an open interval $I \Subset(0,+\infty)$, and $-1<\sigma<1$.

Theorem 6.6. If $R>0$ is large enough the following holds: for all $T>0$, all $N \geq 0$ and all

$$
a \in \mathscr{S}_{\text {hyp }}\left(\Gamma_{\iota}^{ \pm}(R, V, I, \sigma)\right),
$$

we can find

$$
a_{0}(t), \ldots, a_{N}(t) \in \mathscr{Y}_{\text {hyp }}\left(\Phi^{t}(\operatorname{supp}(a))\right) \quad \text { for } 0 \leq \pm t \leq T,
$$

such that, for all $0 \leq \pm t \leq T$ and all $0<h \leq 1$,

$$
\left\|e^{-i t h P} \widehat{O p}_{\iota}(a) e^{i t h P}-\sum_{k=0}^{N} h^{k} \widehat{O p}_{\iota}\left(a_{k}(t)\right)\right\|_{L^{2}(M, \widehat{d G}) \rightarrow L^{2}(\mathcal{M}, \widehat{d G})} \leq C_{N, T, a} h^{N+1} .
$$


This theorem is basically well known. Here the main point is to check (6-39), namely that $a_{0}(t), \ldots$, $a_{N}(t)$ lie in $\mathscr{B}_{\mathrm{hyp}}\left(\Phi^{t}(\operatorname{supp}(a))\right)$. Notice that, by Corollary 3.10, $\Phi^{t}(\operatorname{supp}(a))$ is contained in the same chart as $a$ in which it is therefore sufficient to work.

Using the group property, it is sufficient to prove the result when $T$ is small enough (depending only on $V, I, \sigma)$. To check this point, we choose open sets $V_{1}, V_{2}$ such that $V \Subset V_{1} \Subset V_{2} \Subset V_{\iota}^{\prime}$. Then, for some $C>0$ and all $R$ large enough,

$$
\begin{aligned}
\Phi^{t}\left(\Gamma_{\iota}^{ \pm}(R, V, I, \sigma)\right) & \subset \Gamma_{\iota}^{ \pm}\left(R-C, V_{1}, I, \sigma\right), & & \pm t \geq 0, \\
\Phi^{t}\left(\Gamma_{\iota}^{ \pm}\left(R-C, V_{1}, I, \sigma\right)\right) & \subset \Gamma_{\iota}^{ \pm}\left(R-2 C, V_{2}, I, \sigma\right), & & \pm t \geq 0 .
\end{aligned}
$$

This follows from Corollary 3.10 and the fact that $\rho^{t}$ can be assumed to be nondecreasing, using (3-22). Thus, it is sufficient to prove (6-40) for $0 \leq \pm t \leq \varepsilon$ with $\varepsilon>0$ small enough independent of $a \in$ $\mathscr{S}_{\text {hyp }}\left(\Gamma_{l}^{ \pm}\left(R-C, V_{1}, I, \sigma\right)\right)$. Indeed, if this holds, it holds for $a$ satisfying (6-38) and

$$
e^{i \varepsilon h P} \widehat{O p}_{\iota}(a) e^{-i \varepsilon h P}-\sum_{k=0}^{N} h^{k} \widehat{O p}_{\iota}\left(a_{k}(\varepsilon)\right)+h^{N+1} R_{N}(h, \varepsilon)
$$

with $R_{N}(h, \varepsilon)$ uniformly bounded on $L^{2}(\mathcal{M}, \widehat{d G})$ and $a_{k}(\varepsilon) \in \mathscr{Y}_{\text {hyp }}\left(\Gamma_{\iota}^{ \pm}\left(R-C, V_{1}, I, \sigma\right)\right)$, with $a_{k}(\varepsilon)$ supported in $\Phi^{\varepsilon}(\operatorname{supp}(a))$ more precisely. Conjugating the expression above by $e^{-i \varepsilon h P}$ and then applying the same result with $a_{k}(\varepsilon)$ instead of $a$ we can write

$$
e^{i 2 \varepsilon h P} \widehat{O P}_{\iota}(a) e^{-2 i \varepsilon h P}-\sum_{k=0}^{N} h^{k} \widehat{O P}_{\iota}\left(a_{k}(2 \varepsilon)\right)+h^{N+1} R_{N}(h, 2 \varepsilon),
$$

where $a_{k}(2 \varepsilon)$ is supported in $\Phi^{2 \varepsilon}(\operatorname{supp}(a))$, which is still contained in $\Gamma_{l}^{ \pm}\left(R-C, V_{1}, I, \sigma\right)$, and thus allows one to iterate the procedure.

The interest of considering small times is justified by the following lemma.

Lemma 6.7. Fix $V_{1}, I, \sigma$ as above. For some $R_{1}>0$ large enough and $\varepsilon>0$ small enough,

$$
\left|D_{\text {hyp }}^{j \alpha k \beta}\left(\left(\Phi^{t}\right)^{-1}-\operatorname{Id}_{2 n}\right)\right| \leq C_{j \alpha k \beta} \quad \text { on } \Phi^{t}\left(\Gamma_{\iota}^{ \pm}\left(R_{1}, V_{1}, I, \sigma\right)\right),
$$

for all $0 \leq \pm t \leq \varepsilon$.

Proof. Using the identity

$$
d\left(\Phi^{t}-\operatorname{Id}_{2 n}\right)=\int_{0}^{t} d H_{p}\left(\Phi^{s}\right) d \Phi^{s} d s
$$

and Proposition 3.8, we have $\left|d\left(\Phi^{t}-\mathrm{Id}_{2 n}\right)\right| \lesssim|t|$ hence $\left|\left(d \Phi^{t}\right)^{-1}\right| \lesssim 1$ on $\Gamma_{l}^{ \pm}\left(R_{1}, V_{1}, I, \sigma\right)$ if $R_{1}$ is large enough and $t$ is small enough. We then obtain the result by applying $D_{\text {hyp }}^{j \alpha k \beta}$ to $\Phi^{t} \circ\left(\Phi^{t}\right)^{-1}$ and using the Faà di Bruno formula. For instance, if $j=k=|\alpha|=0$ and $|\beta|=1$, we have

$$
d \Phi_{\mid\left(\Phi^{t}\right)^{-1}}^{t} e^{r} \partial_{\eta}^{\beta}\left(\left(\Phi^{t}\right)^{-1}-\operatorname{Id}_{2 n}\right)=\left(\operatorname{Id}_{2 n}-d \Phi_{\mid\left(\Phi^{t}\right)^{-1}}^{t}\right) e^{r} \partial_{\eta}^{\beta} \operatorname{Id}_{2 n}
$$

where, using Proposition 3.8, the right-hand side is bounded for this is simply $e^{r} \partial_{\eta}^{\beta}\left(\operatorname{Id}_{2 n}-\Phi^{t}\right)$ evaluated at $\left(\Phi^{t}\right)^{-1}$. Higher-order derivatives are studied similarly by iteration, using Lemma 3.6. 
Naturally, $\left(\Phi^{t}\right)^{-1}$ is the reverse Hamiltonian flow, namely flowing $\Phi^{t}\left(\Gamma_{\iota}^{ \pm}\left(R_{1}, V_{1}, I, \sigma\right)\right)$ back to $\Gamma_{\iota}^{ \pm}\left(R_{1}, V_{1}, I, \sigma\right)$. More precisely, for $0 \leq \pm t \leq \varepsilon$,

$$
\frac{d}{d t}\left(\Phi^{t}\right)^{-1}(r, \theta, \rho, \eta)=-H_{p}\left(\left(\Phi^{t}\right)^{-1}(r, \theta, \rho, \eta)\right) \quad \text { for }(r, \theta, \rho, \eta) \in \Phi^{ \pm \varepsilon}\left(\Gamma_{\iota}^{ \pm}\left(R_{1}, V_{1}, I, \sigma\right)\right) .
$$

We prefer to keep the notation $\left(\Phi^{t}\right)^{-1}$ on $\Phi^{t}\left(\Gamma_{l}^{ \pm}\left(R_{1}, V_{1}, I, \sigma\right)\right)$ rather than using $\Phi^{-t}$, since we have only studied $\Phi^{t}$ for $t \geq 0$ on outgoing areas and $t \leq 0$ on incoming areas.

We have essentially all the tools needed to solve the transport equations considered in the next lemma.

Lemma 6.8. There exists $C>0$ such that, for all $R$ large enough, the following holds: for any $a_{\mathrm{ini}} \in$ $\mathscr{S}_{\text {hyp }}\left(\Gamma_{\iota}^{ \pm}(R, V, I, \sigma)\right)$ and any bounded family $(f(t))_{0 \leq \pm t \leq \varepsilon}$ of $\mathscr{S}_{\mathrm{hyp}}\left(\Gamma_{\iota}^{ \pm}\left(R-C, V_{1}, I, \sigma\right)\right)$, smooth with respect to $t$ and such that

$$
\operatorname{supp}(f(t)) \subset \Phi^{t}\left(\operatorname{supp}\left(a_{\text {ini }}\right)\right),
$$

the function defined for $0 \leq \pm t \leq \varepsilon$ by

$$
a(t):= \begin{cases}a_{\mathrm{ini}} \circ\left(\Phi^{t}\right)^{-1}+\int_{0}^{t} f(s) \circ \Phi^{s} \circ\left(\Phi^{t}\right)^{-1} d s & \text { on } \Phi^{t}(\operatorname{supp}(a)), \\ 0 & \text { outside, }\end{cases}
$$

is smooth and satisfies

$$
\partial_{t} a(t)+\{p, a(t)\}=f(t), \quad a(0)=a_{\text {ini }}
$$

Furthermore

$$
(a(t))_{0 \leq \pm t \leq \varepsilon} \text { is bounded in } \mathscr{Y}_{\text {hyp }}\left(\Gamma_{\iota}^{ \pm}\left(R-C, V_{1}, I, \sigma\right)\right) \text {. }
$$

In (6-43), we consider $\Gamma_{l}^{ \pm}\left(R-C, V_{1}, I, \sigma\right)$ for it is independent of $t$ but, by construction, $a(t)$ is supported in the smaller region $\Phi^{t}(\operatorname{supp}(a))$.

Proof. To check the smoothness of $a_{0}(t)$ it suffices to see that $a_{\text {ini }} \circ\left(\Phi^{t}\right)^{-1}$ and $f(s) \circ\left(\Phi^{t-s}\right)^{-1}$ are defined and smooth in a neighborhood of $\Phi^{t}(\operatorname{supp}(a))$, while they vanish on the complement of $\Phi^{t}(\operatorname{supp}(a))$ (relatively to the neighborhood). Indeed $\left(\Phi^{t}\right)^{-1}$ is defined on $\Phi^{t}\left(\Gamma_{l}^{ \pm}\left(R-C, V_{1}, I, \sigma\right)\right)$ and if $(r, \theta, \rho, \eta)$ belongs to $\Phi^{t}\left(\Gamma_{\iota}^{ \pm}\left(R-C, V_{1}, I, \sigma\right)\right)$ but doesn't belong to $\Phi^{t}(\operatorname{supp}(a))$, then $a_{\text {circ }} \circ$ $\left(\Phi^{t}\right)^{-1}(r, \theta, \rho, \eta)=0$; otherwise, $\left(\Phi^{t}\right)^{-1}(r, \theta, \rho, \eta)$ should belong to $\operatorname{supp}(a)$ and thus $(r, \theta, \rho, \eta)$ should belong to $\Phi^{t}(\operatorname{supp}(a))$. Similarly,

$$
\int_{0}^{t} f(s) \circ \Phi^{s} \circ\left(\Phi^{t}\right)^{-1}(r, \theta, \rho, \eta) d s
$$

must vanish, otherwise there would be $s$ between 0 and $t$ such that $\Phi^{s} \circ\left(\Phi^{t}\right)^{-1}(r, \theta, \rho, \eta) \in \Phi^{s}(\operatorname{supp}(a))$ implying that $(r, \theta, \rho, \eta) \in \Phi^{t}(\operatorname{supp}(a))$. Then (6-42) follows directly from (6-41) and (6-43) follows from Lemma 6.7.

Proof of Theorem 6.6. By Lemma 6.8, the solutions of the transport equations (6-42) belong to the set $\mathscr{S}_{\text {hyp }}\left(\Gamma_{\iota}^{ \pm}\left(R-C, V_{1}, I, \sigma\right)\right)$. The proof is then standard; see [Robert 1987], for instance.

Proof of Proposition 2.24. We start by choosing $\varepsilon>0$ and $\delta>0$ according to Proposition 2.16 with $\underline{t}=t_{\mathrm{WKB}}$. Then, using (2-26), (2-37), (2-38) and Theorem 6.6, it is straightforward to show that, for all $T \geq t_{\mathrm{WKB}}$ and all $N \geq 0$,

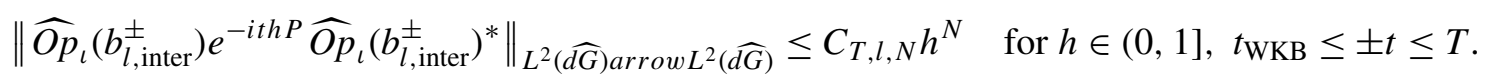


It is therefore sufficient to show the existence of $T$ large enough such that

$$
\left\|\widehat{O p}_{\iota}\left(b_{l, \text { inter }}^{ \pm}\right) e^{-i t h P} \widehat{O p}_{\iota}\left(b_{l, \text { inter }}^{ \pm}\right)^{*}\right\|_{L^{2}(\widehat{d G}) \text { arrow } L^{2}(\widehat{d G})} \leq C_{l, N} h^{N} \quad \text { for } h \in(0,1], T \leq \pm t \leq 2 h^{-1} \text {. }
$$

For simplicity we consider positive times and set $B=\widehat{O p}_{\iota}\left(b_{l, \text { inter }}^{+}\right)$. For $T$ to be chosen, we write

$$
e^{-i t h P} B^{*}=e^{-i(t-T) h P} B(T)^{*} e^{-i T h P}, \quad B(T)=e^{-i T h P} B e^{i T h P} .
$$

As above, we may write

$$
B(T)^{*}=\sum_{k \leq N} h^{k} \widehat{O p}_{\iota}\left(b_{k}^{*}(T)\right)+h^{N+1} B_{N}(h),
$$

with $B_{N}(h)$ uniformly bounded on $L^{2}(\mathcal{M}, \widehat{d G})$ and

$$
b_{k}^{*}(T) \in \mathscr{Y}_{\text {hyp }}\left(\Phi^{T}\left(\operatorname{supp}\left(b_{l, \text { inter }}^{+}\right)\right)\right) \subset \mathscr{Y}_{\text {hyp }}\left(\Phi^{T}\left(\Gamma_{l, \text { inter }}^{+}(\varepsilon, \delta ; l)\right)\right) .
$$

By (2-57), for all $\tilde{\varepsilon}>0$, we can choose $T_{\tilde{\varepsilon}}$ large enough that $\Phi^{T}\left(\Gamma_{\iota \text {,inter }}^{+}(\varepsilon, \delta ; l)\right) \subset \Gamma_{\iota, s}^{+}\left(\tilde{\varepsilon}^{9}\right)$. Thus, if $\tilde{\varepsilon}$ is small enough, Theorem 5.1 allows one to write, for $t \geq T_{\tilde{\varepsilon}}$,

$$
e^{-i\left(t-T_{\tilde{\varepsilon}}\right) h P} \widehat{O p}_{\iota}\left(b_{k}^{*}\left(T_{\tilde{\varepsilon}}\right)\right)=\Psi_{\iota}^{*}\left(J_{h}^{+}\left(\tilde{a}_{\tilde{\varepsilon}}(h)\right) e^{-i\left(t-T_{\tilde{\varepsilon}}\right) h D_{r}^{2}} J_{h}^{+}\left(\tilde{b}_{\tilde{\varepsilon}}(h)\right)^{*}\right)\left(\Psi_{\iota}^{-1}\right)^{*}+h^{N} R_{N}(t, h),
$$

with $R_{N}(t, h)$ uniformly bounded on $L^{2}(\mathcal{M}, \widehat{d G})$ for $h \in(0,1]$ and $0 \leq t-T_{\tilde{\varepsilon}} \leq 2 h^{-1}$, and

$$
\tilde{a}_{\tilde{\varepsilon}}(h) \in \mathscr{T}_{\text {hyp }}\left(\Gamma_{l, s}^{+}(\tilde{\varepsilon})\right) .
$$

We will therefore get (6-44) with $T=T_{\tilde{\varepsilon}}$ if we choose $\tilde{\varepsilon}$ small enough such that, for all $N$,

$$
\left\|b_{l, \text { inter }}^{+}\left(r, \theta, h D_{r}, h D_{\theta}\right) J_{h}^{+}\left(\tilde{a}_{\tilde{\varepsilon}}(h)\right)\right\|_{L^{2}\left(\mathbb{R}^{n}\right) \rightarrow L^{2}\left(\mathbb{R}^{n}\right)} \leq C_{N} h^{N} .
$$

By the standard composition rule between pseudodifferential and Fourier integral operators (see [Robert 1987]), $b_{l, \text { inter }}^{+}\left(r, \theta, h D_{r}, h D_{\theta}\right) J_{h}^{+}\left(\tilde{a}_{\tilde{\varepsilon}}(h)\right)$ is the sum of an operator with norm of order $h^{N}$ and of Fourier integral operators with amplitudes vanishing outside the support of

$$
b_{l, \text { inter }}^{+}\left(r, \theta, \partial_{r} S_{+}, \partial_{\theta} S_{+}\right) \tilde{a}_{\tilde{\varepsilon}}(r, \theta, \rho, \eta, h),
$$

where $S_{+}=S_{+}(r, \theta, \rho, \eta)$ is the phase defined in Proposition 4.9. It is therefore sufficient to show that, for $\tilde{\varepsilon}$ small enough, the support of the amplitude above is empty. Indeed, on this support we have

$$
\frac{\partial_{r} S_{+}}{p\left(r, \theta, \partial_{r} S_{+}, \partial_{\theta} S_{+}\right)^{1 / 2}} \leq 1-(\varepsilon / 2)^{2}, \quad \frac{\rho}{p(r, \theta, \rho, \eta)^{1 / 2}}>1-\tilde{\varepsilon}^{2} .
$$

Furthermore, by Proposition 4.9, we also have

$$
\left|\partial_{r} S_{+}-r\right|+\left|\partial_{\theta} S_{+}-\eta\right| \lesssim \tilde{\varepsilon}^{2}
$$

on $\Gamma_{l, s}^{+}(\tilde{\varepsilon})$, where $\tilde{a}_{\tilde{\varepsilon}}(h)$ is supported. Since $p$ is bounded from above and from below on $\Gamma_{l, s}^{+}(\tilde{\varepsilon})$, we obtain, for all $\tilde{\varepsilon}$ small enough,

$$
\frac{\rho}{p(r, \theta, \rho, \eta)^{1 / 2}} \leq 1-(\varepsilon / 2)^{2}+C \tilde{\varepsilon}^{2} \leq 1-(\varepsilon / 4)^{2},
$$

which is clearly incompatible with the second condition of (6-45). 


\section{Dispersion estimates}

In this section, we prove Propositions 2.20 and 2.22, using respectively the parametrices given in Theorems 5.1 and 6.1. The dispersion estimates will basically follow from the stationary phase theorem, applied to the kernels of these parametrices which are oscillatory integrals. The principle is thus quite simple. One needs however to check some technical points essentially due to the noncompactness of the manifold and, more precisely, to the nonuniform ellipticity of the symbol of the Laplacian.

Here is some heuristic in the case of the Isozaki-Kiada parametrix. We have to consider oscillatory integrals with phases whose model is

$$
\left(r-r^{\prime}\right) \rho+\left(\theta-\theta^{\prime}\right) \cdot \eta-t \rho^{2}+\left(e^{-2 r}-e^{-2 r^{\prime}}\right) \frac{|\eta|^{2}}{4 \rho},
$$

where $r, r^{\prime}, \theta, \theta^{\prime}$ are parameters and $\rho, \eta$ the integration variables. Due to the localization of the amplitudes, we may also assume that $(r, \theta, \rho, \eta)$ and $\left(r^{\prime}, \theta^{\prime}, \rho, \eta\right)$ belong to strongly outgoing/incoming area. The critical point satisfies (assuming that it is unique)

$$
\begin{array}{r}
r-r^{\prime}-2 t \rho-\left(e^{-2 r}-e^{-2 r^{\prime}}\right) \frac{|\eta|^{2}}{4 \rho^{2}}=0, \\
\theta-\theta^{\prime}+\left(e^{-2 r}-e^{-2 r^{\prime}}\right) \frac{\eta}{2 \rho}=0,
\end{array}
$$

where one should also keep in mind that $e^{-r} \eta$ and $e^{-r} \eta^{\prime}$ are small since the amplitudes are supported in strongly outgoing/incoming areas. In particular, $\rho$ is close to $\pm p^{1 / 2}$ and thus is far from 0 . By (7-1), one obtains at the critical point that, as expected,

$$
r \approx r^{\prime}+2 t \rho=r^{\prime}+2|t \rho|
$$

where $t \rho=|t \rho|$ by the sense of time considered in outgoing/incoming areas. This in turn shows that

$$
\theta-\theta^{\prime} \approx e^{-2 r^{\prime}}\left(1-e^{-2 t \rho}\right) \frac{\eta}{\rho}
$$

In Proposition 7.2, we check that this intuition is correct, and we improve the localization around critical points in Proposition 7.6. To use the stationary phase theorem, one needs to check the nondegeneracy of the phase. Using the change of variable $\xi=e^{-r} \eta^{\prime}$, the phase is changed into

$$
\left(r-r^{\prime}\right) \rho+e^{r^{\prime}}\left(\theta-\theta^{\prime}\right) \cdot \xi-t \rho^{2}+\left(e^{-2\left(r-r^{\prime}\right)}-1\right) \frac{|\xi|^{2}}{4 \rho}
$$

and its hessian becomes

$$
t\left\{\left(\begin{array}{cc}
-2 & \\
0 & \frac{e^{-2\left(r-r^{\prime}\right)}-1}{2 t \rho}
\end{array}\right)-\frac{e^{-2\left(r-r^{\prime}\right)}-1}{2 t \rho}\left(\begin{array}{cc}
0 & \xi / \rho \\
\xi / \rho & 0
\end{array}\right)\right\} .
$$

Since $\xi$ is small, the second matrix is small compared to the first one. When $t$ is not too large, the entry $\left(e^{-2\left(r-r^{\prime}\right)}-1\right) /(2 t \rho)$ is bounded from above and below (recall $\left.(7-3)\right)$ and the phase is thus nondegenerate. 
This is made more rigorous in Proposition 7.11. When $t$ becomes large the hessian matrix is basically equivalent to

$$
\left(\begin{array}{cc}
-2 t & \\
0 & -1 /(2 \rho)
\end{array}\right)
$$

which is again nondegenerate but will contribute apparently only through a factor $|t|^{-1 / 2}$ in the stationary phase theorem. However, recalling the change of variable $e^{-r^{\prime}} \eta=\xi$ whose Jacobian is $e^{r^{\prime}(n-1)}$, and using the two factors $e^{-(n-1) r^{\prime} / 2}, e^{-(n-1) r / 2}$ on both sides of the kernel (written with respect to $d G$ rather than $\widehat{d G})$, we get a factor of the form $e^{(n-1)\left(r^{\prime}-r\right) / 2}$ which decays exponentially in $t$ by (7-3) and provides (much more than) the missing $|t|^{-(n-1) / 2}$ decay. This is made more rigorous in Proposition 7.12.

The aim of the following subsection is to justify this intuition. In particular, to justify the above approximations (e.g. the precise meaning of (7-3) or the smallness of the second matrix in (7-4)) we need to be in an asymptotic regime given by a certain (small) parameter: in the Isozaki-Kiada case, the relevant parameter is $\varepsilon$ (the size of the strongly outgoing/incoming areas) and, in the WKB case, it is the range of time.

7A. Stationary and nonstationary phase estimates. For simplicity, we drop the index $\iota$ from the notation, including in outgoing/incoming areas. In both Isozaki-Kiada and WKB parametrices, we have to consider oscillatory integrals of the form

$$
(2 \pi h)^{-n} \iint e^{\frac{i}{h} \Phi^{ \pm}\left(t, r, \theta, r^{\prime}, \theta^{\prime}, \rho, \eta\right)} A^{ \pm}\left(t, r, \theta, r^{\prime}, \theta^{\prime}, \rho, \eta\right) d \rho d \eta .
$$

For the Isozaki-Kiada parametrix, the amplitude is independent of $t$ and of the form

$$
A_{\mathrm{IK}}^{ \pm}\left(t, r, \theta, r^{\prime}, \theta^{\prime}, \rho, \eta\right)=a^{ \pm}(r, \theta, \rho, \eta) \overline{b^{ \pm}\left(r^{\prime}, \theta^{\prime}, \rho, \eta\right)}
$$

with

$$
a^{ \pm} \in \mathscr{Y}_{\text {hyp }}\left(\Gamma_{\mathrm{s}}^{ \pm}(\varepsilon)\right) \text { and } b^{ \pm} \in \mathscr{Y}_{\text {hyp }}\left(\Gamma_{\mathrm{s}}^{ \pm}\left(\varepsilon^{3}\right)\right),
$$

with $\varepsilon>0$ small to be fixed. The phase reads

$$
\Phi_{\mathrm{IK}}^{ \pm}\left(t, r, \theta, r^{\prime}, \theta^{\prime}, \rho, \eta\right)=S_{ \pm, \varepsilon}(r, \theta, \rho, \eta)-t \rho^{2}-S_{ \pm, \varepsilon}\left(r^{\prime}, \theta^{\prime}, \rho, \eta\right),
$$

where $S_{ \pm, \varepsilon}$ is defined in Proposition 4.10. We recall that it coincides with $S_{ \pm}$on $\Gamma_{\mathrm{s}}^{ \pm}(\varepsilon)$ (hence on $\Gamma_{\mathrm{s}}^{ \pm}\left(\varepsilon^{3}\right)$ too), where $S_{ \pm}$is given by Proposition 4.9. We can therefore freely replace $S_{ \pm, \varepsilon}$ by $S_{ \pm}$, or more generally by any other continuation of $S_{ \pm}$outside $\Gamma_{\mathrm{s}}^{ \pm}(\varepsilon)$. Here we have $0 \leq \pm t \leq 2 h^{-1}$. The integral (7-5) is well defined for $\left(r, \theta, r^{\prime}, \theta^{\prime}\right) \in \mathbb{R}^{2 n}$ but, using (7-6), we can assume that

$$
r \geq \varepsilon^{-1}, \quad \theta \in V_{\varepsilon}, \quad r^{\prime} \geq \varepsilon^{-3}, \quad \theta^{\prime} \in V_{\varepsilon^{3}} .
$$

The first goal of this section is to prove that, if $\varepsilon$ is small enough, we can use stationary phase estimates.

The second goal is to show a similar result for the WKB parametrix, using $t_{\mathrm{WKB}}$ as small parameter (see Theorem 6.1). In this case, we have to consider

$$
A_{\mathrm{WKB}}^{ \pm}\left(t, r, \theta, r^{\prime}, \theta^{\prime}, \rho, \eta\right)=a^{ \pm}(t, r, \theta, \rho, \eta),
$$

where, for $V_{2} \Subset \psi_{\iota}\left(U_{l}\right), I_{2} \Subset(0,+\infty), \sigma_{2} \in(-1,1)$, some $R_{2}>0$ large enough and some $t_{\mathrm{WKB}}>0$,

$$
\left(a^{ \pm}(t)\right)_{0 \leq \pm t \leq t_{\mathrm{WKB}}} \text { is bounded in } \mathscr{S}_{\mathrm{hyp}}\left(\Gamma^{ \pm}\left(R_{2}, V_{2}, I_{2}, \sigma_{2}\right)\right) .
$$


In particular, we can assume that

$$
r \geq R_{2}, \quad \theta \in V_{2}
$$

The phase is of the form

$$
\Phi_{\mathrm{WKB}}^{ \pm}\left(t, r, \theta, r^{\prime}, \theta^{\prime}, \rho, \eta\right)=\Sigma^{ \pm}(t, r, \theta, \rho, \eta)-r^{\prime} \rho-\theta^{\prime} \cdot \eta,
$$

and we refer to Theorem 6.1 for more details. We only recall here that the phase $\Sigma^{ \pm}$is defined on $\left[0, \pm t_{\mathrm{WKB}}\right] \times \mathbb{R}^{2 n}$ and solves the eikonal equation (6-3) on $\left[0, \pm t_{\mathrm{WKB}}\right] \times \Gamma^{ \pm}\left(R_{3}, V_{3}, I_{3}, \sigma_{3}\right)$, with $\Gamma^{ \pm}\left(R_{2}, V_{2}, I_{2}, \sigma_{2}\right) \subset \Gamma^{ \pm}\left(R_{3}, V_{3}, I_{3}, \sigma_{3}\right)$. Here again, the condition (7-8) implies that we can freely modify $\Sigma^{ \pm}$outside $\Gamma^{ \pm}\left(R_{2}, V_{2}, I_{2}, \sigma_{2}\right)$.

Below, we will use the notation $\Phi^{ \pm}$(resp. $A^{ \pm}$) either for $\Phi_{\mathrm{IK}}^{ \pm}$or $\Phi_{\mathrm{WKB}}^{ \pm}$(resp. $A_{\mathrm{IK}}^{ \pm}$or $A_{\mathrm{WKB}}^{ \pm}$), as long as a single analysis for both cases will be possible. For convenience we also define

$$
0 \leq \pm t \leq T(h):= \begin{cases}2 h^{-1} & \text { for Isozaki-Kiada } \\ t_{\mathrm{WKB}} & \text { for WKB }\end{cases}
$$

In the next lemma, we summarize the basic properties of $A^{ \pm}$and $\Phi^{ \pm}$needed to get a first nonstationary phase result. For simplicity, we set $\partial^{\gamma}=\partial_{r}^{j} \partial_{\theta}^{\alpha} \partial_{r^{\prime}}^{j^{\prime}} \partial_{\theta^{\prime}}^{\alpha^{\prime}} \partial_{\rho}^{k} \partial_{\eta}^{\beta}$.

Lemma 7.1. In each case, for all $|\gamma| \geq 0$, the amplitude satisfies

$$
\left|\partial^{\gamma} A^{ \pm}\left(t, r, \theta, r^{\prime}, \theta^{\prime}, \rho, \eta\right)\right| \leq C_{\gamma}
$$

for all

$$
\left(r, \theta, r^{\prime}, \theta^{\prime}, \rho, \eta\right) \in \mathbb{R}^{3 n}, \quad h \in(0,1], \quad 0 \leq \pm t \leq T(h),
$$

and we may assume that the phase satisfies

$$
\left|\partial^{\gamma}\left(\Phi^{ \pm}\left(t, r, \theta, r^{\prime}, \theta^{\prime}, \rho, \eta\right)-\left(r-r^{\prime}\right) \rho-\left(\theta-\theta^{\prime}\right) \cdot \eta\right)\right| \leq C_{\gamma}\langle t\rangle,
$$

under the condition (7-12) too. In particular, for all $|\gamma| \geq 1$,

$$
\left|\partial^{\gamma} \partial_{\rho} \Phi^{ \pm}\left(t, r, \theta, r^{\prime}, \theta^{\prime}, \rho, \eta\right)\right| \leq C_{\gamma}\langle t\rangle
$$

under the condition (7-12).

Proof. If $A^{ \pm}=A_{\mathrm{IK}}^{ \pm}$, (7-11) follows easily from Definition 2.2, (7-6), (7-8) and the time independence of $A_{\mathrm{IK}}^{ \pm}$. If $A^{ \pm}=A_{\mathrm{WKB}}^{ \pm},(7-11)$ is a direct consequence of (7-8). For the phase, Proposition 4.10 shows that $\Phi_{\mathrm{IK}}^{ \pm}-\left(r-r^{\prime}\right) \rho-\left(\theta-\theta^{\prime}\right) \cdot \eta$ is the sum of a function $f \in C_{b}^{\infty}\left(\mathbb{R}^{3 n}\right)$ with $-t \rho^{2}$; similarly, by Lemma 7.5, $\Phi_{\mathrm{WKB}}^{ \pm}-\left(r-r^{\prime}\right) \rho-\left(\theta-\theta^{\prime}\right) \cdot \eta$ is the sum of some $f \in C_{b}^{\infty}\left(\mathbb{R}^{3 n}\right)$ with $-t p(r, \theta, \rho, \eta)$. Since the amplitude is compactly supported with respect to $\rho$ and $p(r, \theta, \rho, \eta)$, we may replace $\Phi_{\mathrm{IK}}^{ \pm}$by $\left(r-r^{\prime}\right) \rho-\left(\theta-\theta^{\prime}\right)$. $\eta+f-t \rho^{2} \chi_{1}(\rho)$ and $\Phi_{\mathrm{WKB}}^{ \pm}$by $\left(r-r^{\prime}\right) \rho-\left(\theta-\theta^{\prime}\right) \cdot \eta+f-t p(r, \theta, \rho, \eta) \chi_{1}(p(r, \theta, \rho, \eta))$, for some $\chi_{1} \in C_{0}^{\infty}(\mathbb{R})$. This implies (7-13) and completes the proof.

Now choose $\chi_{1} \in C_{0}^{\infty}(-1,1), \chi_{2} \in C_{0}^{\infty}\left(\mathbb{R}^{n-1}\right)$, both equal to 1 near 0 and define, for any $c_{1}, c_{2}>0$,

$$
A_{c_{1}, c_{2}}^{ \pm}=\chi_{1}\left(\frac{\partial_{\rho} \Phi^{ \pm}}{c_{1}\langle t\rangle}\right) \chi_{2}\left(\frac{\partial_{\eta} \Phi^{ \pm}}{c_{2}}\right) A^{ \pm} .
$$


Let $E^{ \pm}(t, h)$ be the operator with Schwartz kernel (7-5) and $E_{c_{1}, c_{2}}^{ \pm}(t, h)$ the operator with Schwartz kernel

$$
(2 \pi h)^{-n} \iint e^{\frac{i}{h} \Phi^{ \pm}\left(t, r, \theta, r^{\prime}, \theta^{\prime}, \rho, \eta\right)} A_{c_{1}, c_{2}}^{ \pm}\left(t, r, \theta, r^{\prime}, \theta^{\prime}, \rho, \eta\right) d \rho d \eta,
$$

for $h \in(0,1]$ and $0 \leq \pm t \leq T(h)$.

Proposition 7.2 (Semiclassical finite speed of propagation). For all $c_{1}, c_{2}>0$ and all $N \geq 0$, we have

$$
\left\|E^{ \pm}(t, h)-E_{c_{1}, c_{2}}^{ \pm}(t, h)\right\|_{L^{2}\left(\mathbb{R}^{n}\right) \rightarrow L^{2}\left(\mathbb{R}^{n}\right)} \leq C_{N, A, \Phi, c_{1}, c_{2}} h^{N} \quad \text { for } h \in(0,1], 0 \leq \pm t \leq T(h) .
$$

Moreover, if $c_{1}$ is small enough, there exists $C \geq 0$, independent of $\pm t \in[0, T(h)]$ and of $c_{2}>0$, such that

$$
r^{\prime}-r \leq C
$$

on the support of $A_{c_{1}, c_{2}}^{ \pm}$.

Proof. The kernel of $E^{ \pm}(t, h)-E_{c_{1}, c_{2}}^{ \pm}(t, h)$ is an oscillatory integral similar to (7-15) with amplitude

$$
A^{ \pm}-A_{c_{1}, c_{2}}^{ \pm}=\left(1-\chi_{1}\left(\frac{\partial_{\rho} \Phi^{ \pm}}{c_{1}\langle t\rangle}\right)\right) \chi_{2}\left(\frac{\partial_{\eta} \Phi^{ \pm}}{c_{2}}\right) A^{ \pm}+\left(1-\chi_{2}\left(\frac{\partial_{\eta} \Phi^{ \pm}}{c_{2}}\right)\right) A^{ \pm} .
$$

On the support of the second term of the right-hand side, we integrate by part $M$ times with

$$
\frac{h}{i\left|\partial_{\eta} \Phi^{ \pm}\right|^{2}} \partial_{\eta} \Phi^{ \pm} \cdot \partial_{\eta} \text {. }
$$

All derivatives of $\partial_{\eta} \Phi^{ \pm} /\left|\partial_{\eta} \Phi^{ \pm}\right|^{2}$ are bounded since $t$ is bounded in the WKB case and $\partial^{\gamma} \partial_{\eta} \Phi_{\mathrm{IK}}^{ \pm}$is independent of $t$ and bounded for $|\gamma| \geq 1$. On the support of the first term, integrate by part $M$ times with

$$
\frac{h}{i \partial_{\rho} \Phi^{ \pm}} \partial_{\rho} .
$$

Using (7-14), we have, on the support of the first term, $\left|\partial^{\gamma}\left(1 / \partial_{\rho} \Phi^{ \pm}\right)\right| \lesssim 1$, for all $\gamma$. Thus, using also (7-11), we end up in both cases with an integral of the form

$$
h^{M-n} \iint e^{\frac{i}{h} \Phi^{ \pm}\left(t, r, \theta, r^{\prime}, \theta^{\prime}, \rho, \xi\right)} B^{ \pm}\left(t, r, \theta, r^{\prime}, \theta^{\prime}, \rho, \xi\right) d \rho d \xi
$$

with $B^{ \pm}(t, \cdot)$ bounded in $C_{b}^{\infty}\left(\mathbb{R}^{3 n}\right)$, for $0 \leq \pm t \leq T(h)$. We then interpret this integral as the kernel of a pseudodifferential operator with symbol $h^{M} \exp \left(i\left(\Phi^{ \pm}-\left(r-r^{\prime}\right) \rho-\left(\theta-\theta^{\prime}\right) \cdot \eta\right) / h\right) B^{ \pm}$(in the spirit of Lemma 5.4). By the Calderón-Vaillancourt Theorem and (7-13), its operator norm has order $h^{M}(\langle t\rangle / h)^{n_{0}}$, for some universal $n_{0}$ depending only on $n$. Thus we get (7-16) by choosing $M=N+2 n_{0}$.

To prove the second statement, we consider separately the two cases. For the WKB parametrix, $t$ is bounded. Thus, by (7-13), $\partial_{\rho} \Phi_{\mathrm{WKB}}^{ \pm}-\left(r-r^{\prime}\right)$ is bounded and since $\left|\partial_{\rho} \Phi_{\mathrm{WKB}}^{ \pm}\right| \lesssim c_{1}\langle t\rangle$, on the support of $A_{\mathrm{WKB}, c_{1}, c_{2}}^{ \pm}, r-r^{\prime}$ must be bounded too. For the Isozaki-Kiada parametrix, as long as $t$ belongs to a bounded set the same argument holds. We may therefore assume that $\pm t \geq T$ with $T>0$ a fixed large constant. We then exploit two facts: first, for some $c>0$, we have $c< \pm \rho<c^{-1}$ and $t \rho \geq 0$ on the support of $A_{\mathrm{IK}}^{ \pm}$. Second, $f^{ \pm}:=\Phi_{\mathrm{IK}}^{ \pm}-\left(r-r^{\prime}\right) \rho-\left(\theta-\theta^{\prime}\right) \cdot \eta+t \rho^{2}$ is independent of $t$ and bounded, together with all its derivatives on the support of $A_{\mathrm{IK}}^{ \pm}$. Then

$$
\partial_{\rho} \Phi_{\mathrm{IK}}^{ \pm}=r-r^{\prime}-2 t \rho+\partial_{\rho} f^{ \pm}
$$


hence, on the support of $\chi_{1}\left(\partial_{\rho} \Phi_{\mathrm{IK}}^{ \pm} / c_{1}\langle t\rangle\right)$, we have

$$
r-r^{\prime} \geq-c_{1}\langle t\rangle+2 t \rho-\partial_{\rho} f^{ \pm}
$$

If $c_{1}$ is small enough and $T$ large enough, we have $2 t \rho-c_{1}\langle t\rangle \geq 0$ for $t \geq T$. This completes the proof.

Remark. It is clear from the proof that the constant $C$ in (7-17) is uniform with respect to $\varepsilon>0$ small in the Isozaki-Kiada case (recall that the amplitudes depend respectively on $t$ and $\varepsilon$ for the WKB and the IK parametrices).

From now on, we fix $c_{1}>0$ small enough that (7-17) holds.

Proposition 7.3 (Dispersion estimate for times $\leq h$ ). For all $c_{2}>0$, and still with $\gamma_{n}=\frac{n-1}{2}$, we have

$$
\left\|e^{-\gamma_{n} r} E_{c_{1}, c_{2}}^{ \pm}(t, h) e^{-\gamma_{n} r}\right\|_{L^{1}\left(\mathbb{R}^{n}\right) \rightarrow L^{\infty}\left(\mathbb{R}^{n}\right)} \leq C_{A, \Phi, c_{2}}|h t|^{-n / 2} \quad \text { for } 0< \pm t \leq \min (T(h), h) .
$$

Note that the condition $\pm t \leq \min (T(h), h)$ is essentially the condition $\pm t \leq h$. We have put it under this form only because of those $h$ such that $h \geq t_{\mathrm{WKB}}$. This will not modify the rest of the analysis. Furthermore, the latter $h$ correspond to bounded frequencies and their contribution to the Strichartz estimates can be treated by Sobolev embeddings.

Proof. In the Isozaki-Kiada case, both $e^{-r^{\prime}} \eta=\xi$ and $e^{-r} \eta$ are supported in a compact set. In the WKB one, $e^{-r} \eta$ is compactly supported but, using (7-17), this also implies that $e^{-r^{\prime}} \eta$ is compactly supported. Therefore, in both cases, the change of variable $e^{-r^{\prime}} \eta=\xi$ shows that the kernel of $E_{c_{1}, c_{2}}^{ \pm}(t, h)$ is an integral of the form

$$
h^{-n} e^{(n-1) r^{\prime}} \int e^{\frac{i}{h} \Phi^{ \pm}\left(t, r, \theta, r^{\prime}, \theta^{\prime}, \rho, e^{r^{\prime}} \xi\right)} B^{ \pm}\left(t, \theta, r^{\prime}, \theta^{\prime}, \rho, \xi\right) d \rho d \xi,
$$

with $B^{ \pm}$bounded on $[0, \pm T(h)] \times \mathbb{R}^{3 n}$ and supported in a region where $|\rho|+|\xi| \lesssim 1$. The kernel of $e^{-\gamma_{n} r} E_{c_{1}, c_{2}}^{ \pm}(t) e^{-\gamma_{n} r}$ is then simply obtained by multiplying the integral above by $e^{-\gamma_{n}\left(r+r^{\prime}\right)}$, so its modulus is controlled by $h^{-n} e^{\gamma_{n}\left(r^{\prime}-r\right)} \lesssim|h t|^{-n / 2}$, by (7-17) and the fact that $0< \pm t \leq h$. This completes the proof.

To prove the dispersion estimates for $h \leq \pm t \leq T(h)$ we need to analyze the phases more precisely.

In the following lemma and its proof, we shall use the notation (3-4).

Lemma 7.4. For all (fixed) $\tilde{\varepsilon}>0$ small enough, we can find a family of real-valued functions $\left(\varphi_{ \pm, \varepsilon}^{\mathrm{st}}\right)_{0<\varepsilon \ll 1}$ such that

$$
\begin{gathered}
\varphi_{ \pm, \varepsilon}^{\mathrm{st}}=\varphi_{ \pm}=\varphi_{ \pm, \varepsilon} \quad \text { on } \Gamma_{\mathrm{s}}^{ \pm}(\varepsilon), \\
\varphi_{ \pm, \varepsilon}^{\mathrm{st}} \in \mathscr{Y}_{\mathrm{hyp}}\left(\Gamma_{\mathrm{s}}(\tilde{\varepsilon})\right),
\end{gathered}
$$

and that, if we set

$$
R_{ \pm, \varepsilon}(r, \theta, \rho, \eta)=\varphi_{ \pm, \varepsilon}^{\mathrm{st}}(r, \theta, \rho, \eta)-\frac{q_{0}\left(\theta, e^{-r} \eta\right)}{4 \rho}
$$

the following holds for $j+|\alpha| \leq 1$ :

$$
\sup _{\substack{(r, \theta, \eta) \in \mathbb{R}^{2 n-1} \\ \pm \rho \in\left[\frac{1}{4}, 4\right]}}\left|\left(e^{r} \partial_{\eta}\right)^{\beta} \partial_{r}^{j} \partial_{\theta}^{\alpha} \partial_{\rho}^{k} R_{ \pm, \varepsilon}(r, \theta, \rho, \eta)\right| \leq \begin{cases}C \varepsilon^{\tau / 2} & \text { if } k+|\beta| \leq 2, \\ C_{\varepsilon j \alpha k \beta} & \text { if } k+|\beta| \geq 3,\end{cases}
$$

where $\tau$, the decay rate in (1-8), satisfies (1-9). 
Proof. Using (4-35) and Taylor's formula, we can write

$$
\varphi_{ \pm}(r, \theta, \rho, \eta)=\int_{0}^{ \pm \infty} e^{-4 t \rho} q\left(r+2 t \rho, \theta, e^{-r} \eta\right) d t+\sum_{|\gamma|=3} a_{\gamma}(r, \theta, \rho, \eta)\left(e^{-r} \eta\right)^{\gamma}
$$

with $a_{\gamma} \in \mathscr{P}_{\mathrm{hyp}}\left(\Gamma_{\mathrm{s}}^{ \pm}\left(\varepsilon_{0}\right)\right)$ for some fixed $\varepsilon_{0}>0$. Therefore,

$$
\varphi_{ \pm}(r, \theta, \rho, \eta)-\frac{q_{0}\left(\theta, e^{-r} \eta\right)}{4 \rho}=\int_{0}^{ \pm \infty} e^{-4 t \rho} q_{1}\left(r+2 t \rho, \theta, e^{-r} \eta\right) d t+\sum_{|\gamma|=3} a_{\gamma}(r, \theta, \rho, \eta)\left(e^{-r} \eta\right)^{\gamma}
$$

with $q_{1}$ satisfying (3-6). Denote by $R(r, \theta, \rho, \eta)$ the right-hand side of (7-21) and choose $\chi_{1} \in C_{0}^{\infty}(\mathbb{R})$ and $\chi_{2} \in C_{0}^{\infty}\left(\mathbb{R}^{n-1}\right)$ both equal to 1 near 0 . For some $\tilde{\varepsilon}>0$ to be fixed below, choose $\chi_{\tilde{\varepsilon}}^{ \pm}$such that

$$
\chi_{\tilde{\varepsilon}}^{ \pm} \in \mathscr{Y}_{\text {hyp }}\left(\Gamma_{\mathrm{s}}^{ \pm}(\tilde{\varepsilon})\right), \quad \chi_{\tilde{\varepsilon}}^{ \pm} \equiv 1 \text { on } \Gamma_{\mathrm{s}}^{ \pm}\left(\tilde{\varepsilon}^{2}\right),
$$

using Proposition 4.1. (We don't need Proposition 4.4 here, since $\varepsilon^{0}$ will be fixed.) We then claim that, if $\tilde{\varepsilon}$ is small enough (and fixed) and $\varepsilon$ with $\tilde{\varepsilon}^{\prime}$ is small enough too, the function

$$
\varphi_{ \pm, \varepsilon}^{\mathrm{st}}(r, \theta, \rho, \eta):=\frac{q_{0}\left(\theta, e^{-r} \eta\right)}{4 \rho}+R(r, \theta, \rho, \eta) \chi_{\tilde{\varepsilon}}^{ \pm}(r, \theta, \rho, \eta) \chi_{2}\left(e^{-r} \eta / \varepsilon^{1 / 2}\right)\left(1-\chi_{1}\right)\left(\varepsilon^{1 / 2} r\right)
$$

satisfies (7-18), (7-19) and (7-20). Indeed, by choosing $\tilde{\varepsilon}$ small enough, we have $\pm \rho \approx 1$ on the support of $\chi_{\tilde{\varepsilon}}^{ \pm}$, so the integral in (7-21) is exponentially convergent. Furthermore, since

$$
\left|\left(e^{r} \partial_{\eta}\right)^{\beta} \partial_{r}^{j}\left(\left(e^{-r} \eta\right)^{\gamma} \chi_{1}\left(e^{-r} \eta / \varepsilon^{1 / 2}\right)\right)\right| \leq C\left(\varepsilon^{1 / 2}\right)^{|\gamma|-|\beta|},
$$

for all $\gamma$, and using the fact that, if $t \rho \geq 0$ and $r \geq 0$,

$$
\left|\left(e^{r} \partial_{\eta}\right)^{\beta} \partial_{r}^{j} \partial_{\theta}^{\alpha} \partial_{\rho}^{k} q_{1}\left(r+t \rho, \theta, e^{-r} \eta\right)\right| \leq C|t|^{k}\langle r\rangle^{-\tau}\left|e^{-r} \eta\right|^{2-|\beta|},
$$

we get the estimate (7-20). Finally, since $e^{r}|\eta| \lesssim \varepsilon$ and $r \geq \varepsilon$ on $\Gamma_{\mathrm{s}}^{ \pm}(\varepsilon)$, we have (7-18) for all $\varepsilon$ small enough. The property (7-19) is clear thanks to $\chi_{\tilde{\varepsilon}}^{ \pm}$.

In the following lemma, we use the notation of Theorem 6.1 .

Lemma 7.5. We can find a family of real-valued functions $\left(\Sigma_{\mathrm{st}}^{ \pm}(t)\right)_{0 \leq \pm t \leq t_{\mathrm{WKB}}}$ such that

$$
\Sigma_{\mathrm{st}}^{ \pm}(t)=\Sigma^{ \pm}(t) \quad \text { on } \Gamma^{ \pm}\left(R_{2}, V_{2}, I_{2}, \sigma_{2}\right)
$$

and, for all $k, \beta$,

$$
\sup _{\mathbb{R}^{2 n}}\left|\left(e^{r} \partial_{\eta}\right)^{\beta} \partial_{\rho}^{k}\left(\Sigma_{\mathrm{st}}^{ \pm}(t, r, \theta, \rho, \eta)-r \rho-\theta \cdot \eta-t p(r, \theta, \rho, \eta)\right)\right| \leq C_{k \beta} t^{2} .
$$

Proof. Using the function $\chi_{2 \rightarrow 3}^{ \pm}$of Theorem 6.1, the result is straightforward by considering

$$
\begin{aligned}
& \Sigma_{\mathrm{st}}^{ \pm}(t, r, \theta, \rho, \eta) \\
& \quad=\chi_{2 \rightarrow 3}^{ \pm}(r, \theta, \rho, \eta)\left(\Sigma_{0}^{ \pm}(t, r, \theta, \rho, \eta)-r \rho-\theta \cdot \eta-t p(r, \theta, \rho, \eta)\right)+r \rho+\theta \cdot \eta+t p(r, \theta, \rho, \eta)
\end{aligned}
$$

and using (6-4). 
We remark that $\Sigma^{ \pm}$satisfies (6-5) whereas $\Sigma_{\text {st }}^{ \pm}$does not. This was the reason for considering $\Sigma^{ \pm}$first, since the property (6-5) is convenient to prove $L^{2}$ bounds for Fourier integral operators.

The estimates (7-20) and (7-23) show that we have good asymptotics for the phases in certain regimes, namely $\varepsilon \rightarrow 0$ for the Isozaki-Kiada parametrix and $t \rightarrow 0$ for the WKB parametrix. Using Lemmas 7.4 and 7.5, we replace $\varphi_{ \pm, \varepsilon}$ by $\varphi_{ \pm, \varepsilon}^{\text {st }}$ and $\Sigma^{ \pm}$by $\Sigma_{\text {st }}^{ \pm}$in the expression of $\Phi_{\mathrm{IK}}^{ \pm}$and $\Phi_{\mathrm{WKB}}^{ \pm}$, respectively.

To use a single formalism for both cases, we introduce the parameter

$$
\lambda_{\mathrm{st}}:= \begin{cases}\varepsilon & \text { for the Isozaki-Kiada parametrix, } \\ t_{\mathrm{WKB}}^{\mathrm{st}} & \text { for the WKB parametrix, }\end{cases}
$$

where $t_{\mathrm{WKB}}^{\mathrm{st}}>0$ will denote the size of the time interval where $t$ will be allowed to live. Using the change of variable $\xi=e^{-r^{\prime}} \eta$ and factorizing by $t$ in the phase, the integral (7-15) can be written

$$
(2 \pi h)^{-n} e^{2 \gamma_{n} r^{\prime}} \int e^{i \frac{t}{h} \tilde{\phi}_{\lambda_{\mathrm{st}}}^{ \pm}(z, \rho, \xi)} \tilde{A}_{c_{1}, c_{2} \lambda_{\mathrm{st}}}^{ \pm}(z, \rho, \xi) d \rho d \xi,
$$

where $h \in(0,1]$,

$$
\begin{aligned}
\tilde{\Phi}_{\lambda_{\mathrm{st}}}^{ \pm}(y, \rho, \xi) & =\frac{1}{t} \Phi^{ \pm}\left(t, r, \theta, r^{\prime}, \theta^{\prime}, \rho, e^{r^{\prime}} \xi\right), \\
\tilde{A}_{c_{1}, c_{2}, \lambda_{\mathrm{st}}}^{ \pm}(y, \rho, \xi) & =A_{c_{1}, c_{2}}\left(t, r, \theta, r^{\prime}, \theta^{\prime}, \rho, e^{r^{\prime}} \xi\right),
\end{aligned}
$$

and

$$
y=\left(h, t, r, \theta, r^{\prime}, \theta^{\prime}\right),
$$

with $r, r^{\prime}$ satisfying (7-17) and

$$
0< \pm t \leq T\left(h, \lambda_{\mathrm{st}}\right):= \begin{cases}2 h^{-1} & \text { for the Isozaki-Kiada parametrix } \\ t_{\mathrm{WKB}}^{\mathrm{st}} & \text { for the WKB parametrix. }\end{cases}
$$

The kernel of $e^{-\gamma_{n} r} E_{c_{1}, c_{2}}^{ \pm}(t, h) e^{-\gamma_{n} r}$ then becomes

$$
(2 \pi h)^{-n} e^{\gamma_{n}\left(r^{\prime}-r\right)} \int e^{i \frac{t}{h} \tilde{\Phi}_{\lambda_{\mathrm{st}}}^{ \pm}(y, \rho, \xi)} \tilde{A}_{c_{1}, c_{2}, \lambda_{\mathrm{st}}}^{ \pm}(y, \rho, \xi) d \rho d \xi .
$$

Proposition 7.6 (nonstationary phase). There exists $C^{\prime}>0$ such that the condition

$$
\left|\frac{r-r^{\prime}}{t}\right|+e^{r^{\prime}}\left|\frac{\theta-\theta^{\prime}}{t}\right| \geq C^{\prime}
$$

implies that for all $c_{2}>0$, all $N \geq 0$ and all $0<\lambda_{\mathrm{st}} \ll 1$, we can find $C_{c_{2}, N, \lambda_{\mathrm{st}}}$ such that, for all

$$
h \in(0,1], \quad \pm t \in\left[h, T\left(h, \lambda_{\mathrm{st}}\right)\right], \quad \omega \geq 1, \quad\left(r, \theta, r^{\prime}, \theta^{\prime}\right) \in \mathbb{R}^{2 n},
$$

with $r, r^{\prime}$ satisfying (7-17), we have

$$
\left|(2 \pi h)^{-n} e^{\gamma_{n}\left(r^{\prime}-r\right)} \int e^{i \omega \tilde{\Phi}_{\lambda_{\mathrm{st}}}^{ \pm}(y, \rho, \xi)} \tilde{A}_{c_{1}, c_{2}, \lambda_{\mathrm{st}}}^{ \pm}(y, \rho, \xi) d \rho d \xi\right| \leq C_{c_{2}, N, \lambda_{\mathrm{st}}} h^{-n} \omega^{-N}
$$

Proof. For $t \neq 0$, we define

$$
\tilde{\Phi}_{t}^{\text {free }}:=\frac{r-r^{\prime}}{t} \rho+e^{r^{\prime}} \frac{\theta-\theta^{\prime}}{t} \cdot \xi
$$


Then

$$
\nabla_{\rho, \xi} \tilde{\Phi}_{t}^{\text {free }}=\left(\frac{r-r^{\prime}}{t}, e^{r^{\prime}} \frac{\theta-\theta^{\prime}}{t}\right)
$$

We then start with the case of $\Phi_{\mathrm{WKB}}^{ \pm}$. By Lemma 7.5 and (7-17), $\nabla_{\rho, \xi}\left(\tilde{\Phi}_{\lambda_{\mathrm{st}}}-\tilde{\Phi}_{t}^{\text {free }}\right)$ is a function of $\left(t, r, \theta, r^{\prime}, \rho, \xi\right)$ which is bounded on the support of the amplitude, as well as all its derivatives in $\rho, \xi$, uniformly with respect to $\left(t, r, \theta, r^{\prime}\right)$. Therefore, if $C^{\prime}$ is large enough, we have

$$
\left|\nabla_{\rho, \xi} \tilde{\Phi}_{\lambda_{\mathrm{st}}}\right| \gtrsim\left|\frac{r-r^{\prime}}{t}\right|+e^{r^{\prime}}\left|\frac{\theta-\theta^{\prime}}{t}\right|,
$$

and the result follows from standard integrations by parts. Note that, here, we have not used the smallness of $\lambda_{\text {st }}$ (i.e., of $t$ ). We shall use it for the case of $\Phi_{\mathrm{IK}}^{ \pm}$which we now consider. Since $\pm \rho \in\left[\frac{1}{4}, 4\right]$ on the support of the amplitude if $\varepsilon=\lambda_{\text {st }}$ is small enough, Lemma 7.4 and Taylor's formula imply that

$$
\nabla_{\rho, \xi}\left(\tilde{\Phi}_{\lambda_{\mathrm{st}}}-\tilde{\Phi}_{t}^{\mathrm{free}}\right)=(-2 \rho, 0)+\nabla_{\rho, \xi} \frac{q_{0}\left(\theta, e^{r^{\prime}-r} \xi\right)-q_{0}\left(\theta^{\prime}, \xi\right)}{t \rho}+\varepsilon_{\varepsilon}(y, \rho, \xi)\left(\frac{r-r^{\prime}}{t}, \frac{\theta-\theta^{\prime}}{t}\right),
$$

where $\varepsilon_{\varepsilon}(y, \rho, \xi)$ and all its derivatives in $\rho$, $\xi$ go to 0 as $\varepsilon \rightarrow 0$, uniformly with respect to $y$ (see (7-26)) with $r, r^{\prime}$ satisfying (7-17) and $( \pm \rho, \xi) \in\left[\frac{1}{4}, 4\right] \times \mathbb{R}^{n-1}$. Furthermore, using (7-17) and the fact that $|\xi| \lesssim \varepsilon^{3}$ on the support of the amplitude, we have

$$
\left|\nabla_{\rho, \xi} \frac{q_{0}\left(\theta, e^{r^{\prime}-r} \xi\right)-q_{0}\left(\theta^{\prime}, \xi\right)}{t \rho}\right| \lesssim \varepsilon^{3}\left|\left(\frac{r-r^{\prime}}{t}, \frac{\theta-\theta^{\prime}}{t}\right)\right|
$$

thus, using that $r^{\prime} \geq 0$ on the support of the amplitude, we have (7-28) if $\varepsilon$ is small enough. In addition, for all $k+|\beta| \geq 2$, we also have

$$
\left|\partial_{\rho}^{k} \partial_{\xi}^{\beta} \tilde{\Phi}_{\lambda_{\mathrm{st}}}\right| \lesssim\left|\left(\frac{r-r^{\prime}}{t}, \frac{\theta-\theta^{\prime}}{t}\right)\right|
$$

on the support of the amplitude, using (7-17). The result then follows again from integrations by parts.

We next state a convenient form of the stationary phase theorem with parameters; the demonstration a simple adaptation of the proof of [Hörmander 1983, Theorem 7.7.5] — is given in Appendix A for completeness.

Proposition 7.7 (Stationary phase theorem). Let $\Omega$ be a set and

$$
f: \mathbb{R}^{n} \times \Omega \ni(x, y) \mapsto f(x, y) \in \mathbb{R}
$$

a function, smooth with respect to $x$ and such that

$$
\operatorname{Hess}_{x}[f](x, y)=S(y)+R(x, y) \quad \text { for }(x, y) \in \mathbb{R}^{n} \times \Omega,
$$

with $S(y)$ a symmetric nonsingular matrix such that

$$
\left|S(y)^{-1}\right| \lesssim 1 \quad \text { for } y \in \Omega
$$

and $R(x, y)$ a symmetric matrix such that

$$
\left\|S(y)^{-1} R(x, y)\right\| \leq \frac{1}{2} \quad \text { for }(x, y) \in \mathbb{R}^{n} \times \Omega,
$$


where $\|\cdot\|$ is the Euclidean matrix norm. Then there exists $N \geq 0$ such that, for all $K \Subset \mathbb{R}^{n}$, there exists $C_{K}>0$ satisfying

$$
\left|\int e^{i \omega f(x, y)} u(x) d x\right| \leq C_{K} \omega^{-n / 2} \sup _{|\alpha| \leq N}\left\|\partial^{\alpha} u\right\|_{L^{\infty}(K)} \sup _{2 \leq|\alpha| \leq N}\left(\sup _{x \in K}\left|\partial^{\alpha} f(x, y)\right|+1\right)^{N},
$$

for all $y \in \Omega$, all $u \in C_{0}^{\infty}(K)$ and all $\omega \geq 1$.

For the WKB parametrix, we shall use this proposition fairly directly by considering

$$
\Omega_{\mathrm{WKB}}^{ \pm}\left(t_{\mathrm{WKB}}^{\mathrm{st}}\right)=\left\{\left(h, t, r, \theta, r^{\prime}, \theta^{\prime}\right): h \in(0,1], \quad\left|\frac{r-r^{\prime}}{t}\right| \leq C^{\prime}, h \leq \pm t \leq t_{\mathrm{WKB}}^{\mathrm{st}}\right\}
$$

Notice in particular that $r-r^{\prime}$ is bounded on $\Omega_{\mathrm{WKB}}\left(t_{\mathrm{WKB}}^{\mathrm{st}}\right)$.

Proposition 7.8 (Dispersion estimate for the $\mathrm{WKB}$ parametrix). Fix $c_{2}>0$. There exists $t_{\mathrm{WKB}}^{\mathrm{st}}>0$ small enough such that, for all $y=\left(h, t, r, \theta, r^{\prime}, \theta^{\prime}\right) \in \Omega_{\mathrm{WKB}}^{ \pm}\left(t_{\mathrm{WKB}}^{\mathrm{st}}\right)$ and all $\omega \geq 1$, we have

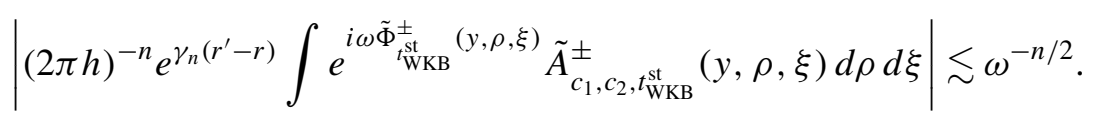

Proof. This is a straightforward application of Proposition 7.7 since, using (7-23), we have

$$
\operatorname{Hess}_{\rho, \xi}\left[\tilde{\Phi}_{t_{\mathrm{WKB}}^{\mathrm{st}}}\right]=\left(\begin{array}{cc}
2 & 0 \\
0 & \operatorname{Hess}_{\eta}(q)
\end{array}\right)+\mathcal{O}\left(t_{\mathrm{WKB}}^{\mathrm{st}}\right)
$$

where the first matrix of the right-hand side satisfies (7-30) by the uniform ellipticity of $q$. The conclusion is then clear since all derivatives, in $\rho, \xi$, of $\tilde{A}_{t_{\mathrm{WKB}}^{\mathrm{st}}}^{ \pm}$are bounded, as well as those of $\tilde{\Phi}_{t_{\mathrm{WKB}}^{\mathrm{st}}}$ of order at least 2 , on the support of the amplitude.

To be in position to use Proposition 7.7 for the Isozaki-Kiada parametrix, we still need two lemmas.

Lemma 7.9 (Sharper localization for IK). Let $\chi_{0} \in C_{0}^{\infty}(\mathbb{R})$ be equal to 1 near 0 and set

$$
\chi_{\varepsilon}(y, \rho)=\chi_{0}\left(\varepsilon^{-\tau / 4}\left(2 \rho-\frac{r-r^{\prime}}{t}\right)\right) .
$$

Then, for all $\varepsilon>0$ small enough, all $N \geq 0$ and all $c_{2}>0$, there exists $C_{c_{2}, N, \varepsilon}$ such that, for all

$$
h \in(0,1], \quad \pm h \leq t \leq 2 h^{-1}, \quad \omega \geq 1,
$$

and all $\left(r, \theta, r^{\prime}, \theta^{\prime}\right) \in \mathbb{R}^{2 n}$ satisfying (7-7) and such that

$$
\left|\frac{r-r^{\prime}}{t}\right|+e^{r^{\prime}}\left|\frac{\theta-\theta^{\prime}}{t}\right| \leq C^{\prime}
$$

we have

$$
\left|(2 \pi h)^{-n} e^{\gamma_{n}\left(r^{\prime}-r\right)} \int e^{i \omega \tilde{\Phi}_{\varepsilon}^{ \pm}(y, \rho, \xi)}\left(1-\chi_{\varepsilon}(y, \rho)\right) \tilde{A}_{c_{1}, c_{2}, \varepsilon}^{ \pm}(y, \rho, \xi) d \rho d \xi\right| \leq C_{c_{2}, N, \varepsilon} h^{-n} \omega^{-N} .
$$

Proof. By the same analysis as in the proof of Proposition 7.6, using Lemma 7.4 and (7-33), we may write

$$
\tilde{\Phi}_{\varepsilon}^{ \pm}(y, \rho, \xi)=\frac{r-r^{\prime}}{t} \rho-\rho^{2}+R_{\varepsilon}^{ \pm}(y, \rho, \xi),
$$


where, on the support of the amplitude, we have

$$
\left|\partial_{\rho} R_{\varepsilon}^{ \pm}\right| \lesssim \varepsilon^{\tau / 2}, \quad\left|\partial_{\rho}^{k} \partial_{\xi}^{\beta} R_{\varepsilon}^{ \pm}\right| \lesssim 1
$$

for $k+|\beta| \geq 1$. On the other hand, on the support of $\left(1-\chi_{\varepsilon}(y, \rho)\right)$ we also have, for some $c>0$,

$$
\frac{r-r^{\prime}}{t}-2 \rho \geq c \varepsilon^{\tau / 4} \quad \text { or } \quad \frac{r-r^{\prime}}{t}-2 \rho \leq-c \varepsilon^{\tau / 4} .
$$

Therefore, if $\varepsilon$ is small enough,

$$
\left|\partial_{\rho} \tilde{\Phi}_{\varepsilon}^{ \pm}(y, \rho, \xi)\right| \gtrsim \varepsilon^{\tau / 4},
$$

on the support of the amplitude and the result follows from integrations by parts in $\rho$.

Basically, the interest of the localization (7-34) is to replace $\frac{1}{4} \rho$ in (7-20) by $2 t /\left(r-r^{\prime}\right)$ up to a small error. We implement this idea as follows. By Lemma 7.9, we can replace $\tilde{A}_{c_{1}, c_{2}, \varepsilon}^{ \pm}(y, \rho, \xi)$ in (7-25) by

$$
\chi_{\varepsilon}(y, \rho) \tilde{A}_{c_{1}, c_{2}, \varepsilon}^{ \pm}(y, \rho, \xi) .
$$

If $\varepsilon$ is small enough, we have $\pm \rho \in\left[\frac{1}{4}, 4\right]$ on the support of $\tilde{A}_{c_{1}, c_{2}, \varepsilon}^{ \pm}$hence, for some $c>0$,

$$
c|t| \leq r-r^{\prime} \leq c^{-1}|t|,
$$

on the support of (7-34), which is stronger than (7-17). Furthermore, the condition (7-33) together with (7-7) implies that we may assume that $\left|\theta-\theta^{\prime}\right| \leq C^{\prime} e^{-\varepsilon^{-3}}|t|$. From now on we fix

$$
c_{2}=\varepsilon .
$$

Thus, by writing

$$
\partial_{\eta} \Phi_{\mathrm{IK}}^{ \pm}=\theta-\theta^{\prime}+\partial_{\eta} \varphi_{ \pm}(r, \theta, \rho, \eta)-\partial_{\eta} \varphi_{ \pm}\left(r^{\prime}, \theta^{\prime}, \rho, \eta\right),
$$

with $\varphi_{ \pm} \in \mathscr{P}_{\mathrm{hyp}}\left(\Gamma^{ \pm}\left(\varepsilon_{2}\right)\right)$, we have $\left|\partial_{\eta} \varphi_{ \pm}(r, \theta, \rho, \eta)\right| \lesssim e^{-r}$ and $\left|\partial_{\eta} \varphi_{ \pm}\left(r^{\prime}, \theta^{\prime}, \rho, \eta\right)\right| \lesssim e^{-r^{\prime}}$ on the support of the amplitude. By (7-7), we have for instance $\left|\partial_{\eta} \Phi_{\mathrm{IK}}^{ \pm}-\left(\theta-\theta^{\prime}\right)\right| \leq \varepsilon^{2}$ if $\varepsilon$ is small enough. We may therefore assume that

$$
\left|\theta-\theta^{\prime}\right| \leq C^{\prime \prime} \varepsilon \frac{|t|}{\langle t\rangle} .
$$

To be set of parameters for the stationary phase theorem, we will thus choose

$$
\Omega_{\mathrm{IK}}^{ \pm}(\varepsilon)=\left\{\left(h, t, r, \theta, r^{\prime}, \theta^{\prime}\right): h \in(0,1], \pm t \in\left[h, 2 h^{-1}\right] \text { and (7-7), (7-33), (7-35), (7-36) hold }\right\} .
$$

Before applying Proposition 7.7, we still need to modify the phase $\tilde{\Phi}_{\varepsilon}^{ \pm}$outside the support of the new amplitude (7-34).

Lemma 7.10. We can find $\Psi_{\varepsilon}^{ \pm}$smooth and real-valued such that, on the support of (7-34),

$$
\Psi_{\varepsilon}^{ \pm}(y, \rho, \xi)=\tilde{\Phi}_{\varepsilon}^{ \pm}(y, \rho, \xi)
$$

and

$$
\Psi_{\varepsilon}^{ \pm}(y, \rho, \xi)=\frac{r-r^{\prime}}{t} \rho+\frac{\theta-\theta^{\prime}}{t} e^{r^{\prime}} \xi-\rho^{2}-\frac{1-e^{2\left(r^{\prime}-r\right)}}{2\left(r-r^{\prime}\right)} q_{0}\left(\theta^{\prime}, \xi\right)+\psi_{\varepsilon}^{ \pm}(y, \rho, \xi)
$$


where, for all $k+|\beta| \leq 2$,

$$
\sup _{\substack{(\rho, \xi) \in \mathbb{R}^{n} \\ y \in \Omega_{\mathrm{IK}}^{ \pm}(\varepsilon)}}\left|\langle t\rangle^{|\beta| / 2} \partial_{\rho}^{k} \partial_{\xi}^{\beta} \psi_{\varepsilon}^{ \pm}(y, \rho, \xi)\right| \rightarrow 0 \quad \text { as } \varepsilon \rightarrow 0,
$$

and for $|k|+|\beta| \geq 3$,

$$
\sup _{\substack{(\rho, \xi) \in \mathbb{R}^{n} \\ y \in \Omega_{\mathrm{IK}}^{ \pm}(\varepsilon)}}\left|\partial_{\rho}^{k} \partial_{\xi}^{\beta} \psi_{\varepsilon}^{ \pm}(y, \rho, \xi)\right| \leq C_{\varepsilon, k, \beta} .
$$

Proof. We shall basically combine (7-20) with the fact that

$$
\left|2 \rho-\left(r-r^{\prime}\right) / t\right| \lesssim \varepsilon^{\tau / 4},
$$

on the support of (7-34). By Lemma 7.4, the phase reads

$$
\frac{r-r^{\prime}}{t} \rho+\frac{\theta-\theta^{\prime}}{t} e^{r^{\prime}} \xi-\rho^{2}-\frac{q_{0}\left(\theta^{\prime}, \xi\right)-e^{2\left(r^{\prime}-r\right)} q_{0}(\theta, \xi)}{4 \rho t}+\frac{R_{ \pm, \varepsilon}\left(r, \theta, \rho, e^{r^{\prime}} \xi\right)-R_{ \pm, \varepsilon}\left(r^{\prime}, \theta^{\prime}, \rho, e^{r^{\prime}} \xi\right)}{t}
$$

The last term of this sum satisfies the estimates (7-38) and (7-39): for $0< \pm t \leq 1$, it follows from Taylor's formula using (7-33) and Lemma 7.4 with $j+|\alpha|=1$, and for $\pm t \geq 1$ it follows from Lemma 7.4 with $j+|\alpha|=0$. For the term involving $q_{0}$ we write

$$
\frac{1}{4 \rho t}=\frac{1}{2\left(r-r^{\prime}\right)}+\left(\frac{1}{4 \rho t}-\frac{1}{2\left(r-r^{\prime}\right)}\right) \chi_{1}\left(\frac{2 \rho-\left(r-r^{\prime}\right) / t}{\varepsilon^{\tau / 8}}\right),
$$

using (7-40) with $\varepsilon$ small enough and $\chi_{1} \in C_{0}^{\infty}\left(\mathbb{R}^{n-1}\right)$ equal to 1 near 0 , and

$$
q_{0}\left(\theta, e^{r^{\prime}-r} \xi\right)=e^{2\left(r^{\prime}-r\right)} q_{0}\left(\theta^{\prime}, \xi\right)+e^{2\left(r^{\prime}-r\right)}\left(q_{0}(\theta, \xi)-q_{0}\left(\theta^{\prime}, \xi\right)\right) \chi_{2}(\xi),
$$

with $\chi_{2} \in C_{0}^{\infty}\left(\mathbb{R}^{n-1}\right)$ equal to 1 near 0 . We obtain the estimates (7-38) and (7-39) for

using (7-36), and for

$$
\frac{1}{4 \rho t} e^{2\left(r^{\prime}-r\right)}\left(q_{0}(\theta, \xi)-q_{0}\left(\theta^{\prime}, \xi\right)\right) \chi_{2}(\xi)
$$

$$
\left(1-e^{2\left(r^{\prime}-r\right)}\right) q_{0}\left(\theta^{\prime}, \xi\right)\left(\frac{1}{4 \rho t}-\frac{1}{2\left(r-r^{\prime}\right)}\right) \chi_{1}\left(\frac{2 \rho-\left(r-r^{\prime}\right) / t}{\varepsilon^{\tau / 8}}\right)
$$

using (7-35). In both cases, we can freely multiply the functions by a compactly support cutoff in $\rho$ using that $\pm \approx 1$ on the support of the amplitude. This completes the proof.

Proposition 7.11 (bounded times). There exists $\varepsilon_{\mathrm{st}}>0$ such that, for all $T>0$, all $0<\varepsilon \leq \varepsilon_{\mathrm{st}}$, there exists $C_{\varepsilon, T}$ such that, for all

$$
h \in(0,1], \quad h \leq \pm t \leq T, \quad\left(r, \theta, r^{\prime}, \theta^{\prime}\right) \text { satisfying (7-7), (7-35) and (7-36) }
$$

we have

$$
\left|(2 \pi h)^{-n} e^{\gamma_{n}\left(r^{\prime}-r\right)} \int e^{i \frac{t}{h} \tilde{\Phi}_{\varepsilon}^{ \pm}(y, \rho, \xi)} \chi_{\varepsilon}(y, \rho) \tilde{A}_{c_{1}, \varepsilon, \varepsilon}(y, \rho, \xi) d \rho d \xi\right| \leq C_{\varepsilon, T}|h t|^{-n / 2} .
$$


Proof. By Lemma 7.10, we can replace $\tilde{\Phi}_{\varepsilon}^{ \pm}$by $\Psi_{\varepsilon}^{ \pm}$. We then have

$$
\operatorname{Hess}_{\rho, \xi}\left[\Psi_{\varepsilon}^{ \pm}\right]=\left(\begin{array}{cc}
2 & 0 \\
0 & \frac{1-e^{2\left(r^{\prime}-r\right)}}{2\left(r-r^{\prime}\right)} \operatorname{Hess}_{\eta}\left(q_{0}\right)
\end{array}\right)+o(1)
$$

where $o(1) \rightarrow 0$ as $\varepsilon \rightarrow 0$, uniformly with respect to $(\rho, \xi) \in \mathbb{R}^{n}$ and to the parameters satisfying (7-41). Using the upper bound in (7-35) and the boundedness of $t$, the positive number

$$
\frac{1-e^{2\left(r^{\prime}-r\right)}}{2\left(r-r^{\prime}\right)}
$$

belongs to a compact subset of $(0, \infty)$, yielding the condition $(7-30)$. We then conclude by applying Proposition 7.7 .

To obtain (7-42), we have used the boundedness of $e^{\gamma_{n}\left(r^{\prime}-r\right)}$, since $\left|r-r^{\prime}\right|$ was bounded. In principle, the condition (7-35) implies that $e^{\gamma_{n}\left(r^{\prime}-r\right)}$ decays exponentially in time. We shall exploit the latter below.

Proposition 7.12 (Large times). There exists $T>0$ and $\varepsilon_{\mathrm{st}}^{\prime}$ such that, for all $0<\varepsilon \leq \varepsilon_{\mathrm{st}}^{\prime}$, there exists $C_{\varepsilon}$ such that, for all

$$
h \in(0,1], \quad T \leq \pm t \leq 2 h^{-1}, \quad\left(r, \theta, r^{\prime}, \theta^{\prime}\right) \text { satisfying (7-7), (7-35) and (7-36) }
$$

we have

$$
\left|(2 \pi h)^{-n} e^{\gamma_{n}\left(r^{\prime}-r\right)} \int e^{i \frac{t}{h} \tilde{\Phi}_{\varepsilon}^{ \pm}(y, \rho, \xi)} \chi_{\varepsilon}(y, \rho) \tilde{A}_{c_{1}, \varepsilon, \varepsilon}(y, \rho, \xi) d \rho d \xi\right| \leq C_{\varepsilon}|h t|^{-n / 2} .
$$

Proof. Choose $T$ large enough such that, for $t \geq T$ and $r, r^{\prime}$ satisfying (7-35), we have $e^{2\left(r^{\prime}-r\right)} \leq \frac{1}{2}$. To compensate the factor $1 /\left(r-r^{\prime}\right)$ in (7-37) (of order $1 /|t|$ by (7-35)), we consider the new variable $|t|^{1 / 2} \zeta=\xi$. By (7-38), if $\varepsilon$ is small enough, this new phase satisfies the assumptions of Proposition 7.7. In the corresponding estimate given by Proposition 7.7, derivatives of the new amplitude as well as derivatives of the new phase of order at least 3 will grow at most polynomially with respect to $t$. This gives a polynomial growth in $t$ of the coefficient in the stationary phase estimate of Proposition 7.7 but such a growth is controlled by the exponential decay of $e^{\gamma_{n}\left(r^{\prime}-r\right)} \lesssim e^{-c|t|}$. This completes the proof.

7B. Proof of Proposition 2.20. By (2-38), up to a remainder of operator norm of size $h^{n}$ (uniformly in time), we may replace $\widehat{O P}_{\iota}\left(a_{\mathrm{s}}^{ \pm}\right)^{*}$ by a linear combination of operators of the form $\widehat{O P}_{\iota}\left(\tilde{a}_{\mathrm{s}}^{ \pm}\right)$with $\operatorname{supp}\left(\tilde{a}_{\mathrm{s}}^{ \pm}\right) \subset \operatorname{supp}\left(a_{\mathrm{s}}^{ \pm}\right)$. We next apply Theorem 5.1 to order $n+1$ and are left with the study of the Fourier integral operator part. By Proposition 7.2, the amplitude can be modified so that, up to a remainder of operator norm of order $h^{n}$ uniformly in time, we are left with an operator whose kernel $K^{ \pm}\left(r, \theta, r^{\prime}, \theta^{\prime}, t, h\right)$ satisfies

$$
\left|e^{-\gamma_{n} r} K^{ \pm}\left(r, \theta, r^{\prime}, \theta^{\prime}, t, h\right) e^{-\gamma_{n} r^{\prime}}\right| \lesssim|h t|^{-n / 2}, \quad h \in(0,1], 0< \pm t \leq 2 h .
$$

Indeed, for $t \leq h$, this follows from Proposition 7.3 and for $t \geq h$, from Propositions 7.11 and 7.12 with $\omega= \pm t / h$ and also from Proposition 7.6 and Lemma 7.9 with $N \geq n / 2$.

Proof of Proposition 2.22. It is completely similar to the one of Proposition 2.20 by considering times $0 \leq \pm t \leq t_{\mathrm{WKB}}^{\mathrm{st}}$ with $t_{\mathrm{WKB}}^{\mathrm{st}}$ small enough to be in position to use both Theorem 6.1 and Proposition 7.8. 


\section{Appendix A. Control on the range of some diffeomorphisms}

In this section, we prove a proposition implying Lemma 4.7 and (4-54) in Lemma 4.11. For simplicity, we consider the outgoing case only but the symmetric result holds in the incoming one.

Let us define the following conical subset of $T^{*} \mathbb{R}_{+}^{n} \backslash 0$,

$$
\Gamma_{\mathrm{s}-\mathrm{con}}^{+}(\varepsilon)=\left\{(r, \theta, \rho, \eta): r>R(\varepsilon), \theta \in V_{\varepsilon}, \rho>\left(1-\varepsilon^{2}\right)\left(\rho^{2}+q\left(r, \theta, e^{-r} \eta\right)\right)^{1 / 2}\right\},
$$

which is the cone generated by $\Gamma_{\mathrm{s}}^{+}(\varepsilon)$.

Proposition A.1. Assume that, for some $0<\bar{\varepsilon}<\frac{1}{4}$, we are given a family of maps $\left(\Psi^{t}\right)_{t \geq 0}$ defined on $\Gamma_{s-c o n}^{+}(\bar{\varepsilon})$, of the form

$$
\Psi^{t}(r, \theta, \rho, \eta)=\left(r, \theta, \underline{\rho}^{t}(r, \theta, \rho, \eta), \underline{\eta}^{t}(r, \theta, \rho, \eta)\right) \in \mathbb{R}^{2 n},
$$

satisfying, for all $r>R(\bar{\varepsilon}), \theta \in V_{\bar{\varepsilon}}, \rho>\left(1-\bar{\varepsilon}^{2}\right) p^{1 / 2}, t \geq 0$ and $\lambda>0$,

$$
\begin{aligned}
\left(\underline{\rho}^{t}, \underline{\eta}^{t}\right)(r, \theta, \lambda \rho, \lambda \eta) & =\lambda\left(\underline{\rho}^{\lambda t}, \underline{\eta}^{\lambda t}\right)(r, \theta, \rho, \eta), \\
\left(\underline{\rho}^{t}, \underline{\eta}^{t}\right)(r, \theta, \rho, 0) & =(\rho, 0),
\end{aligned}
$$

and such that

$$
\left.\left(\underline{\rho}^{t}-\rho\right)_{t \geq 0} \text { and (the components of }\right)\left(\underline{\eta}^{t}-\eta\right)_{t \geq 0} \text { are bounded in } \mathscr{B}_{\mathrm{hyp}}\left(\Gamma_{\mathrm{s}}^{+}(\bar{\varepsilon})\right) \text {. }
$$

Then, there exists $0<\tilde{\varepsilon} \leq \bar{\varepsilon}$ such that, for all $t \geq 0$ and all $0<\varepsilon \leq \tilde{\varepsilon}$, $\Psi^{t}$ is a diffeomorphism from $\Gamma_{\mathrm{s}}^{+}(\varepsilon)$ onto its range and

$$
\Gamma_{\mathrm{s}}^{+}\left(\varepsilon^{3}\right) \subset \Psi^{t}\left(\Gamma_{\mathrm{s}}^{+}(\varepsilon)\right), \quad t \geq 0, \quad 0<\varepsilon \leq \tilde{\varepsilon} .
$$

Lemma 4.7 is indeed a consequence of Proposition A.1 since Proposition 3.8, (3-35) and (4-15) show that (A-2), (A-3) and (A-4) hold with $\left(\underline{\rho}^{t}, \underline{\eta}^{t}\right)=\left(\rho^{t}, \eta^{t}\right)$. Similarly, for Lemma 4.11, we consider

$$
\left(\underline{\rho}^{t}, \underline{\eta}^{t}\right)(r, \theta, \rho, \eta):=\left(\underline{\rho}_{+}, \underline{\eta}_{+}\right)(r, \theta, r, \theta, \rho, \eta)
$$

which is independent of $t$ and satisfies the assumptions (A-2), (A-3), (A-4) by (4-50), Proposition 4.9 and Remark 2 after Proposition 4.9.

To prove the proposition, we need another conical subset of $T^{*} \mathbb{R}_{+}^{n} \backslash 0$ :

$$
\tilde{\Gamma}_{\text {s-con }}^{+}(\varepsilon)=\left\{(r, \theta, \rho, \eta): r>R(\varepsilon), \theta \in V_{\varepsilon}, \rho>\left(1-\varepsilon^{2}\right)\left(\rho^{2}+|\eta|^{2}\right)^{1 / 2}\right\} .
$$

Using the diffeomorphism $F_{\text {hyp }}$ defined by (2-31), we have

$$
F_{\text {hyp }}^{-1}\left(\tilde{\Gamma}_{\text {s-con }}^{+}(\varepsilon)\right)=\left\{(r, \theta, \rho, \eta): r>R(\varepsilon), \theta \in V_{\varepsilon}, \rho>\left(1-\varepsilon^{2}\right)\left(\rho^{2}+\left|e^{-r} \eta\right|^{2}\right)^{1 / 2}\right\} .
$$

The latter is of interest in view of the following lemma.

Lemma A.2. There exists $C>1$ such that, for all $\varepsilon>0$ small enough,

$$
\Gamma_{s-c o n}^{+}(\varepsilon / C) \subset F_{\text {hyp }}^{-1}\left(\tilde{\Gamma}_{s-c o n}^{+}(\varepsilon)\right) \subset \Gamma_{s-c o n}^{+}(C \varepsilon) .
$$


Proof. By (3-7), we have, for some $0<c<1$,

$$
c e^{-2 r}|\eta|^{2} \leq q\left(r, \theta, e^{-r} \eta\right) \leq c^{-1}\left|e^{-r} \eta\right|^{2}, \quad r>R(\varepsilon), \theta \in V_{\varepsilon}, \eta \in \mathbb{R}^{n-1} .
$$

Using (2-55), it suffices to show the existence of $C>1$ satisfying, for all $\varepsilon$ small enough,

$$
c^{-1}\left(1-(\varepsilon / C)^{2}\right)^{-2}\left(1-\left(1-(\varepsilon / C)^{2}\right)^{2}\right) \leq\left(1-\varepsilon^{2}\right)^{-2}\left(1-\left(1-\varepsilon^{2}\right)^{2}\right),
$$

and

$$
\left(1-\varepsilon^{2}\right)^{-2}\left(1-\left(1-\varepsilon^{2}\right)^{2}\right) \leq c\left(1-(C \varepsilon)^{2}\right)^{-2}\left(1-\left(1-(C \varepsilon)^{2}\right)^{2}\right) .
$$

For $\varepsilon \rightarrow 0$, the left-hand side of (A-6) is equivalent to $2 c^{-1}(\varepsilon / C)^{2}$ and the right-hand side to $2 \varepsilon^{2}$. Therefore, (A-6) holds if $c^{-1} / C^{2}<1$ and $\varepsilon$ is small enough. We get (A-7) similarly.

Let us now consider $(1,0)=(1,0, \ldots, 0) \in \mathbb{R}^{n} \backslash 0$. For all $0<\varepsilon<1$, let us denote by $\mathscr{C}^{+}(\varepsilon)$ the cone generated by $B((1,0), \varepsilon)$, namely

$$
\mathscr{C}^{+}(\varepsilon)=\left\{\left.(\lambda \rho, \lambda \eta)\left|\lambda>0,(\rho-1)^{2}+\right| \eta\right|^{2}<\varepsilon^{2}\right\} .
$$

Since $\rho>1-\varepsilon>0$ and $\rho^{2} /\left(\rho^{2}+|\eta|^{2}\right)>1-\varepsilon^{2} /(1-\varepsilon)^{2}$ on $B((1,0), \varepsilon)$, it is then not hard to check that, for all $\varepsilon$ small enough,

$$
\mathscr{C}^{+}\left(\varepsilon^{2} / 4\right) \subset\left\{\rho>\left(1-\varepsilon^{2}\right)\left(\rho^{2}+|\eta|^{2}\right)^{1 / 2}\right\},
$$

and

$$
\left\{\rho>\left(1-\varepsilon^{2}\right)\left(\rho^{2}+|\eta|^{2}\right)^{1 / 2}\right\} \subset \mathscr{C}^{+}(2 \varepsilon),
$$

since, if $\rho>\left(1-\varepsilon^{2}\right)\left(\rho^{2}+|\eta|^{2}\right)^{1 / 2}$ then $(1, \eta / \rho) \in B((1,0), 2 \varepsilon)$, using that $1-\left(1-\varepsilon^{2}\right)^{2}<4 \varepsilon^{2}\left(1-\varepsilon^{2}\right)^{2}$ for $\varepsilon$ small enough. In particular, we obtain

$$
(R(\varepsilon),+\infty) \times V_{\varepsilon} \times \mathscr{C}^{+}\left(\varepsilon^{2} / 4\right) \subset \tilde{\Gamma}_{\mathrm{s}-\mathrm{con}}^{+}(\varepsilon) \subset(R(\varepsilon),+\infty) \times V_{\varepsilon} \times \mathscr{C}^{+}(2 \varepsilon) .
$$

We next recall a standard lemma the simple proof of which we omit.

Lemma A.3. Let $x_{0} \in \mathbb{R}^{n}, \varepsilon>0$ and $f: B\left(x_{0}, \varepsilon\right) \rightarrow \mathbb{R}^{n}$ such that $f\left(x_{0}\right)=x_{0}$ and $f-\mathrm{id}$ is $\frac{1}{2}$ Lipschitz (meaning that $|f(x)-x+y-f(y)| \leq|x-y| / 2)$ on $B\left(x_{0}, \varepsilon\right)$. Then $f$ is injective on $B\left(x_{0}, \varepsilon\right)$ and

$$
B\left(x_{0}, \varepsilon / 2\right) \subset f\left(B\left(x_{0}, \varepsilon\right)\right) .
$$

Proof of Proposition A.1. Set

$$
f_{r, \theta, t}(\rho, \xi)=\left(\underline{\rho}^{t}\left(r, \theta, \rho, e^{r} \xi\right), e^{-r} \underline{\eta}^{t}\left(r, \theta, \rho, e^{r} \xi\right)\right) .
$$

By Lemma 2.4 and (A-4), we have, for $k+|\beta|=2$,

$$
\left|\partial_{\rho}^{k} \partial_{\eta}^{\beta} f_{r, \theta, t}(\rho, \eta)\right| \lesssim 1, \quad t \geq 0,(r, \theta, \rho, \xi) \in F_{\text {hyp }}\left(\Gamma_{\mathrm{s}}^{+}(\bar{\varepsilon})\right),
$$

and, by choosing $\bar{\varepsilon}_{1}$ small enough, we also have

$$
r>R(\bar{\varepsilon}), \quad \theta \in V_{\bar{\varepsilon}}, \quad(\rho, \xi) \in B\left((1,0), \bar{\varepsilon}_{1}\right) \quad \Longrightarrow \quad(r, \theta, \rho, \xi) \in F_{\text {hyp }}\left(\Gamma_{\mathrm{s}}^{+}(\bar{\varepsilon})\right) .
$$


By (A-3) $\partial_{\rho, \xi} f_{r, \theta, t}(\rho, 0)=\operatorname{Id}_{n}$, so (A-9) implies that $f_{r, \theta, t}-\operatorname{Id}_{n}$ is $\frac{1}{2}$-Lipschitz on $B((1,0), 2 \varepsilon)$ for all $\varepsilon$ small enough, all $t \geq 0, r>R(\bar{\varepsilon})$, and $\theta \in V_{\bar{\varepsilon}}$. Therefore, by Lemma A.3,

$$
B((1,0), \varepsilon) \subset f_{t, r, \theta}(B((1,0), 2 \varepsilon)), \quad t \geq 0, r>R(\tilde{\varepsilon}), \theta \in V_{\tilde{\varepsilon}} .
$$

Using (A-2), we can replace the balls in the inclusion above by the cones they generate and, using Lemma A.2 with (A-8), we get

$$
\Gamma_{\text {s-con }}^{+}(\varepsilon / 2 C) \subset \Psi^{t}\left(\Gamma_{\text {s-con }}^{+}\left(2 \sqrt{2} C \varepsilon^{1 / 2}\right)\right), \quad t \geq 0,
$$

for all $\varepsilon$ small enough, with the $C>1$ of Lemma A.2. Since $f_{r, \theta, t}-\operatorname{Id}_{n}$ is $\frac{1}{2}$-Lipschitz on $B((1,0), 2 \varepsilon)$ for all $t \geq 0$, (A-2) implies that it is also $\frac{1}{2}$-Lipschitz on the cone generated by $B((1,0), 2 \varepsilon)$ so $f_{r, \theta, t}$ is injective on this cone. Thus, for all $\varepsilon$ small enough and $t \geq 0, \Psi^{t}$ is injective on $\Gamma_{\text {s-con }}^{+}(\varepsilon)$ and is a diffeomorphism onto its range. By (A-10), we have

$$
\Gamma_{\mathrm{s}}^{+}\left(\varepsilon^{3}\right) \subset \Gamma_{\mathrm{s}-\mathrm{con}}^{+}\left(\varepsilon^{3}\right) \subset \Psi^{t}\left(\Gamma_{\mathrm{s}-\mathrm{con}}^{+}(\varepsilon)\right),
$$

for all $t \geq 0$ and all $\varepsilon$ small enough, so the proof will be completed by showing that, for all $\varepsilon$ small enough and all $t \geq 0$, the following implication holds:

$(r, \theta, \rho, \eta)=\Psi^{t}\left(r, \theta, \rho_{1}, \eta_{1}\right) \in \Gamma_{\mathrm{s}}^{+}\left(\varepsilon^{3}\right)$ with $\left(r, \theta, \rho_{1}, \eta_{1}\right) \in \Gamma_{\mathrm{s}-\mathrm{con}}^{+}(\varepsilon)$

$$
\Longrightarrow p\left(r, \theta, \rho_{1}, \eta_{1}\right) \in\left(\frac{1}{4}-\varepsilon, 4+\varepsilon\right) \text {. }
$$

Assume the first line of (A-11). Using (A-3) at $\left(\rho_{1}, 0\right)$ and the fact that $f_{t, r, \theta}-\mathrm{Id}_{n}$ is $\frac{1}{2}$-Lipschitz, we have

$$
\left|\left(\rho, e^{-r} \eta\right)-\left(\rho_{1}, e^{-r} \eta_{1}\right)\right|=\left|f_{t, r, \theta}\left(\rho_{1}, e^{-r} \eta_{1}\right)-\left(\rho_{1}, e^{-r} \eta_{1}\right)\right| \leq\left|e^{-r} \eta_{1}\right| / 2 .
$$

Therefore $\left|e^{-r} \eta-e^{-r} \eta_{1}\right| \leq\left|e^{-r} \eta_{1}\right| / 2$ and we get $\left|\eta_{1}\right| \leq 2|\eta|$. Since $e^{-r}|\eta| \lesssim \varepsilon^{3}$, (A-12) shows that $\left|\rho-\rho_{1}\right|+\left|e^{-r}\left(\eta-\eta_{1}\right)\right| \lesssim \varepsilon^{3}$ hence that

$$
\left|p\left(r, \theta, \rho_{1}, \eta_{1}\right)-p(r, \theta, \rho, \eta)\right| \lesssim \varepsilon^{3} .
$$

Since $p(r, \theta, \rho, \eta) \in\left(\frac{1}{4}-\varepsilon^{3}, 4+\varepsilon^{3}\right)$, the latter yields (A-11) for $\varepsilon$ small enough.

Proof of Proposition 7.7.

Note first that, for all $y \in \Omega$, the map

$$
\mathbb{R}^{n} \ni x \mapsto \nabla_{x} f(x, y) \in \mathbb{R}^{n}
$$

is a diffeomorphism since, by considering $F(x, y):=S(y)^{-1} \nabla_{x} f(x, y)$ and using (7-29), (7-31) and (7-30), $x \mapsto F(x, y)-x$ is $\frac{1}{2}$ Lipschitz. For all $y \in \Omega$, we denote by $x_{0}=x_{0}(y)$ the unique solution to

$$
\nabla_{x} f\left(x_{0}, y\right)=0 .
$$

Now consider

$$
g(x, y)=f(x, y)-f\left(x_{0}, y\right)-\left\langle\operatorname{Hess}_{x}[f]\left(x_{0}, y\right)\left(x-x_{0}\right), x-x_{0}\right\rangle / 2,
$$

and, for all $s \in[0,1]$,

$$
f_{s}(x, y)=f\left(x_{0}, y\right)+\left\langle\operatorname{Hess}_{x}[f]\left(x_{0}, y\right)\left(x-x_{0}\right), x-x_{0}\right\rangle / 2+\operatorname{sg}(x, y) .
$$


Notice that $f_{1}=f$, that $f_{0}-f\left(x_{0}, y\right)$ is quadratic with respect to $x-x_{0}$ and that

$$
\nabla_{x} f_{s}(x, y)=\left\{S(y)+s \int_{0}^{1} R\left(x_{0}+t\left(x-x_{0}\right), y\right) d t+(1-s) R\left(x_{0}, y\right)\right\}\left(x-x_{0}\right),
$$

by Taylor's formula and (7-29). By (7-30), there exists $c>0$ such that $|S(y) X| \geq 2 c|X|$, for all $X \in \mathbb{R}^{n}$ and all $y \in \Omega$ hence (7-31) implies that

$$
\left|\nabla_{x} f_{s}(x, y)\right| \geq c\left|x-x_{0}(y)\right|, \quad s \in[0,1], \quad(x, y) \in \mathbb{R}^{n} \times \Omega .
$$

Lemma A.4. For all $K \Subset \mathbb{R}^{n}$ and all integer $k \geq 1$, there exists $C>0$ and $N>0$ such that, for all $s \in[0,1]$, all $y \in \Omega$ and all $u$ such that

$$
\begin{array}{rc}
u \in C_{0}^{2 k-1}(K) \cap C^{2 k}\left(\mathbb{R}^{n} \backslash\left\{x_{0}(y)\right\}\right), \\
\partial_{x}^{\alpha} u\left(x_{0}(y)\right)=0, & |\alpha|<2 k, \\
\partial_{x}^{\alpha} u \in L^{\infty}\left(\mathbb{R}^{n}\right), & |\alpha|=2 k,
\end{array}
$$

we have

$$
\left|\int e^{i \omega f_{s}(x, y)} u(x) d x\right| \leq C \omega^{-k} \max _{|\alpha| \leq 2 k}\left\|\partial^{\alpha} u\right\|_{L^{\infty}(K)} \max _{2 \leq|\alpha| \leq 2 k}\left(1+\sup _{x \in K}\left|\partial^{\alpha} f_{s}\right|\right)^{N}, \quad \omega \geq 1 .
$$

Notice that the assumption (A-16) is only a condition near $x_{0}(y)$. It guarantees the boundedness of $\partial^{\alpha} u(x) /\left|x-x_{0}\right|^{2 k-|\alpha|}$.

Proof. We proceed by induction and consider first $k=1$. We would like to integrate by part using the operator $\left|\nabla_{x} f_{s}\right|^{-2} \nabla_{x} f_{s} \cdot \nabla_{x}$ but, since $\nabla_{x} f_{s}$ may vanish on the support of $u$, we consider $L_{\delta}:=$ $\left(\left|\nabla_{x} f_{s}\right|^{2}+\delta\right)^{-1} \nabla_{x} f_{s} \cdot \nabla_{x}$ which satisfies

$$
i \omega \int e^{i \omega f_{s}(x, y)} u(x) d x=\lim _{\delta \downarrow 0} \int\left(L_{\delta} e^{i \omega f_{s}(x, y)}\right) u(x) d x .
$$

We then integrate by part at fixed $\delta>0$, using that

$$
{ }^{t} L_{\delta}=-\frac{1}{\left|\nabla_{x} f_{s}\right|^{2}+\delta}\left\{\nabla_{x} f_{s} \cdot \nabla_{x}+\Delta_{x} f_{s}-\frac{2}{\left|\nabla_{x} f_{s}\right|^{2}+\delta}\left\langle\operatorname{Hess}_{x}\left[f_{s}\right] \nabla_{x} f_{s}, \nabla_{x} f_{s}\right\rangle\right\} .
$$

Since $\left|\Delta_{x} f_{s}(x, y) u(x)\right| \lesssim \max _{|\alpha|=2}\left\|\Delta_{x} f_{s}(\cdot, y)\right\|_{L^{\infty}(K)}\left\|\partial^{\alpha} u\right\|_{L^{\infty}}\left|x-x_{0}(y)\right|^{2}$ and using (A-13), by letting $\delta \downarrow 0$ we get

$$
\left|i \omega \int e^{i \omega f_{s}(x, y)} u(x) d x\right| \leq C \max _{|\alpha| \leq 2}\left\|\partial^{\alpha} u\right\|_{L^{\infty}(K)} \max _{|\alpha|=2}\left(1+\sup _{x \in \mathbb{R}^{n}}\left|\partial^{\alpha} f_{s}\right|\right) .
$$

Here the constant $C$ is independent of $y, u, s$ and $\omega$; it depends only on $K$ and the constant $c$ in (A-13). The result then follows by induction using that

$$
\left|\nabla_{x} f_{s}\right|^{-2}\left\langle\nabla_{x} f_{s}, \partial_{x} u\right\rangle, \quad\left|\nabla_{x} f_{s}\right|^{-2}\left(\Delta_{x} f_{s}\right) u, \quad\left|\nabla_{x} f_{s}\right|^{-4}\left\langle\operatorname{Hess}_{x}\left[f_{s}\right] \nabla_{x} f_{s}, \nabla_{x} f_{s}\right\rangle u
$$

satisfy the assumptions (A-14), (A-15) and (A-16) if $u$ does for $k+1$. 
End of the proof of Proposition 7.7. We next consider $I(s)=\int e^{i \omega f_{s}(x, y)} u(x) d x$ so that, for all $j \in \mathbb{N}_{0}$, we have

$$
I^{(2 j)}(s)=(i \omega)^{2 j} \int e^{i \omega f_{s}(x, y)} g(x, y)^{2 j} u(x) d x .
$$

Since $\partial_{x}^{\alpha}\left(g(x, y)^{2 j}\right)_{\mid x=x_{0}(y)}=0$ for all $|\alpha|<6 j$, Lemma A.4 yields, with $k=3 j \geq n / 2$,

$$
\left|I^{(2 j)}(s)\right| \leq C \omega^{-n / 2} \max _{|\alpha| \leq 6 j}\left\|\partial^{\alpha} u\right\|_{L^{\infty}(K)} \max _{2 \leq|\alpha| \leq 6 j}\left(1+\sup _{x \in \mathbb{R}^{n}}\left|\partial^{\alpha} f_{s}\right|\right)^{N}, \quad s \in[0,1] .
$$

Since $I(1)=\int e^{i \omega f(x, y)} u(x) d x$, the estimate

$$
\left|I(1)-\sum_{l<2 j} I^{(l)}(0) / l !\right| \leq \sup _{s \in[0,1]}\left|I^{(2 j)}(s)\right| /(2 j) !,
$$

reduces the proof to estimating the integrals $I^{(l)}(0)$ whose common phase $f_{0}$ is quadratic, up to a constant term and whose amplitude is $u(x) g(x, y)^{l}$. By Taylor's formula $g(x, y)$ is of order $\left|x-x_{0}(y)\right|^{2}$ so the derivatives of $u(x) g(x, y)^{l}$ may be of order $\left\langle x_{0}(y)\right\rangle^{2 l}$ on which we have no control. By choosing $\tilde{K}$ a bounded neighborhood of $K$ and applying Lemma A.4 to the subset of $\Omega$ on which $x_{0}(y) \notin \tilde{K}$, we can assume that we consider those $y$ for which $x_{0}(y) \in \tilde{K}$. We then use the Lemma 7.7.3 of [Hörmander 1983] on oscillatory integrals with quadratic phases, observing that $\left\|\partial_{x}^{\alpha} g(\cdot, y)^{l}\right\|_{L^{\infty}\left(K_{x}\right)}$ is controlled by (products of) of norms $\left\|\partial_{x}^{\beta} f(\cdot, y)\right\|_{L^{\infty}\left(K_{x}\right)}$ with $|\beta| \geq 2$, since $x$ is bounded on the support of $u$ and $x_{0}(y)$ remains bounded.

\section{Acknowledgments}

It is a pleasure to thank Nikolay Tzvetkov for helpful and stimulating discussions about this paper. The author would also like to emphasize the high quality of the referring process and thanks sincerely the anonymous referees for their suggestions and for the very careful reading of the first version of this paper.

\section{References}

[Anker and Pierfelice 2009] J.-P. Anker and V. Pierfelice, "Nonlinear Schrödinger equation on real hyperbolic spaces", Ann. Inst. H. Poincaré Anal. Non Linéaire 26:5 (2009), 1853-1869. MR 2010m:35416

[Banica 2007] V. Banica, "The nonlinear Schrödinger equation on hyperbolic space", Comm. Partial Differential Equations 32:10-12 (2007), 1643-1677. MR 2008m:35323

[Banica and Duyckaerts 2007] V. Banica and T. Duyckaerts, "Weighted Strichartz estimates for radial Schrödinger equation on noncompact manifolds”, Dyn. Partial Differ. Equ. 4:4 (2007), 335-359. MR 2008j:58036

[Banica et al. 2008] V. Banica, R. Carles, and G. Staffilani, "Scattering theory for radial nonlinear Schrödinger equations on hyperbolic space”, Geom. Funct. Anal. 18:2 (2008), 367-399. MR 2010h:35364

[Bouclet 2006] J.-M. Bouclet, "Resolvent estimates for the Laplacian on asymptotically hyperbolic manifolds", Ann. Henri Poincaré 7:3 (2006), 527-561. MR 2007k:58050 Zbl 1155.58012

[Bouclet 2007] J.-M. Bouclet, "Semi-classical functional calculus on manifolds with ends and weighted $L^{p}$ estimates", 2007. to appear in Ann. Inst. Fourier. arXiv 0711.3583

[Bouclet 2010] J.-M. Bouclet, "Littlewood-Paley decompositions on manifolds with ends", Bull. Soc. Math. France 138:1 (2010), 1-37. MR 2638890 Zbl 1198.42013

[Bouclet and Tzvetkov 2007] J.-M. Bouclet and N. Tzvetkov, "Strichartz estimates for long range perturbations", Amer. J. Math. 129:6 (2007), 1565-1609. MR 2009d:58035 
[Bouclet and Tzvetkov 2008] J.-M. Bouclet and N. Tzvetkov, "On global Strichartz estimates for non-trapping metrics", J. Funct. Anal. 254:6 (2008), 1661-1682. MR 2009d:35039 Zbl 1168.35005

[Bourgain 1993] J. Bourgain, "Fourier transform restriction phenomena for certain lattice subsets and applications to nonlinear evolution equations. I. Schrödinger equations", Geom. Funct. Anal. 3:2 (1993), 107-156. MR 95d:35160a

[Burq et al. 2004] N. Burq, P. Gérard, and N. Tzvetkov, "Strichartz inequalities and the nonlinear Schrödinger equation on compact manifolds", Amer. J. Math. 126:3 (2004), 569-605. MR 2005h:58036

[Cardoso and Vodev 2002] F. Cardoso and G. Vodev, "Uniform estimates of the resolvent of the Laplace-Beltrami operator on infinite volume Riemannian manifolds. II", Ann. Henri Poincaré 3:4 (2002), 673-691. MR 2003j:58054 Zbl 1021.58016

[Cazenave 2003] T. Cazenave, Semilinear Schrödinger equations, Courant Lecture Notes in Math. 10, Courant Institute (NYU), New York, 2003. MR 2004j:35266

[Doi 1996] S.-i. Doi, "Smoothing effects of Schrödinger evolution groups on Riemannian manifolds", Duke Math. J. 82:3 (1996), 679-706. MR 97f:58141

[Froese and Hislop 1989] R. Froese and P. Hislop, "Spectral analysis of second-order elliptic operators on noncompact manifolds", Duke Math. J. 58:1 (1989), 103-129. MR 90k:58234 Zbl 0687.35060

[Ginibre and Velo 1985] J. Ginibre and G. Velo, "The global Cauchy problem for the nonlinear Schrödinger equation revisited", Ann. Inst. H. Poincaré Anal. Non Linéaire 2:4 (1985), 309-327. MR 87b:35150

[Hassell et al. 2006] A. Hassell, T. Tao, and J. Wunsch, "Sharp Strichartz estimates on nontrapping asymptotically conic manifolds", Amer. J. Math. 128:4 (2006), 963-1024. MR 2007d:58053 Zbl 1177.58019

[Helffer and Robert 1983] B. Helffer and D. Robert, "Calcul fonctionnel par la transformation de Mellin et opérateurs admissibles”, J. Funct. Anal. 53:3 (1983), 246-268. MR 85i:47052 Zbl 0524.35103

[Hörmander 1983] L. Hörmander, The analysis of linear partial differential operators, I, Springer, Berlin, 1983.

[Isozaki and Kitada 1985] H. Isozaki and H. Kitada, "Modified wave operators with time-independent modifiers", J. Fac. Sci. Univ. Tokyo Sect. IA Math. 32:1 (1985), 77-104. MR 86j:35125 Zbl 0582.35036

[Keel and Tao 1998] M. Keel and T. Tao, “Endpoint Strichartz estimates”, Amer. J. Math. 120:5 (1998), 955-980. MR 2000d: 35018 Zbl 0922.35028

[Mazzeo and Melrose 1987] R. R. Mazzeo and R. B. Melrose, "Meromorphic extension of the resolvent on complete spaces with asymptotically constant negative curvature", J. Funct. Anal. 75:2 (1987), 260-310. MR 89c:58133 Zbl 0636.58034

[Melrose 1995] R. B. Melrose, Geometric scattering theory, Cambridge University Press, Cambridge, 1995. MR 96k:35129 Zbl 0849.58071

[Pierfelice 2006] V. Pierfelice, "Weighted Strichartz estimates for the radial perturbed Schrödinger equation on the hyperbolic space”, Manuscripta Math. 120:4 (2006), 377-389. MR 2008i:35195

[Pierfelice 2008] V. Pierfelice, "Weighted Strichartz estimates for the Schrödinger and wave equations on Damek-Ricci spaces", Math. Z. 260:2 (2008), 377-392. MR 2009d:35272

[Robbiano and Zuily 2005] L. Robbiano and C. Zuily, Strichartz estimates for Schrödinger equations with variable coefficients, Mém. Soc. Math. Fr. (N.S.) 101-102, Soc. Mat. de France, Paris, 2005. MR 2006i:35047

[Robert 1987] D. Robert, Autour de l'approximation semi-classique, Progress in Mathematics 68, Birkhäuser, Boston, 1987. MR 89g:81016 Zbl 0621.35001

[Staffilani and Tataru 2002] G. Staffilani and D. Tataru, "Strichartz estimates for a Schrödinger operator with nonsmooth coefficients", Comm. Partial Differential Equations 27:7-8 (2002), 1337-1372. MR 2003f:35248

[Strichartz 1977] R. S. Strichartz, "Restrictions of Fourier transforms to quadratic surfaces and decay of solutions of wave equations", Duke Math. J. 44:3 (1977), 705-714. MR 58 \#23577 Zbl 0372.35001

[Tataru 2001] D. Tataru, "Strichartz estimates in the hyperbolic space and global existence for the semilinear wave equation", Trans. Amer. Math. Soc. 353:2 (2001), 795-807. MR 2001k:35218 Zbl 0956.35088

Received 14 Mar 2008. Revised 4 Aug 2009. Accepted 3 Dec 2009.

JEAN-MARC BOUCLET: Jean-Marc.Bouclet@math.univ-lille1.fr

UMR 8524 CNRS, UFR de Mathématiques, Université de Lille 1, 59655 Villeneuve d'Ascq, France 


\section{Analysis \& PDE}

pjm.math.berkeley.edu/apde

EDITORS

EDITOR-IN-CHIEF

Maciej Zworski

University of California

Berkeley, USA

BOARD OF EDITORS

\begin{tabular}{|c|c|c|c|}
\hline Michael Aizenman & $\begin{array}{l}\text { Princeton University, USA } \\
\text { aizenman@math.princeton.edu }\end{array}$ & Nicolas Burq & $\begin{array}{l}\text { Université Paris-Sud 11, France } \\
\text { nicolas.burq@math.u-psud.fr }\end{array}$ \\
\hline Luis A. Caffarelli & $\begin{array}{l}\text { University of Texas, USA } \\
\text { caffarel@ math.utexas.edu }\end{array}$ & un-Yung Alice Chang & $\begin{array}{l}\text { Princeton University, USA } \\
\text { chang@math.princeton.edu }\end{array}$ \\
\hline Michael Christ & $\begin{array}{l}\text { University of California, Berkeley, USA } \\
\text { mchrist@math.berkeley.edu }\end{array}$ & Charles Fefferman & $\begin{array}{l}\text { Princeton University, USA } \\
\text { cf@math.princeton.edu }\end{array}$ \\
\hline Ursula Hamenstaedt & $\begin{array}{l}\text { Universität Bonn, Germany } \\
\text { ursula@math.uni-bonn.de }\end{array}$ & Nigel Higson & $\begin{array}{l}\text { Pennsylvania State Univesity, USA } \\
\text { higson@math.psu.edu }\end{array}$ \\
\hline Vaughan Jones & $\begin{array}{l}\text { University of California, Berkeley, USA } \\
\text { vfr@math.berkeley.edu }\end{array}$ & Herbert Koch & $\begin{array}{l}\text { Universität Bonn, Germany } \\
\text { koch@math.uni-bonn.de }\end{array}$ \\
\hline Izabella Laba & $\begin{array}{l}\text { University of British Columbia, Canada } \\
\text { ilaba@math.ubc.ca }\end{array}$ & Gilles Lebeau & $\begin{array}{l}\text { Université de Nice Sophia Antipolis, France } \\
\text { lebeau@unice.fr }\end{array}$ \\
\hline László Lempert & $\begin{array}{l}\text { Purdue University, USA } \\
\text { lempert@math.purdue.edu }\end{array}$ & Richard B. Melrose & $\begin{array}{l}\text { Massachussets Institute of Technology, USA } \\
\text { rbm@math.mit.edu }\end{array}$ \\
\hline Frank Merle & $\begin{array}{l}\text { Université de Cergy-Pontoise, France } \\
\text { Frank.Merle@u-cergy.fr }\end{array}$ & William Minicozzi II & $\begin{array}{l}\text { Johns Hopkins University, USA } \\
\text { minicozz@ math.jhu.edu }\end{array}$ \\
\hline Werner Müller & $\begin{array}{l}\text { Universität Bonn, Germany } \\
\text { mueller@math.uni-bonn.de }\end{array}$ & Yuval Peres & $\begin{array}{l}\text { University of California, Berkeley, USA } \\
\text { peres@stat.berkeley.edu }\end{array}$ \\
\hline Gilles Pisier & $\begin{array}{l}\text { Texas A\&M University, and Paris } 6 \\
\text { pisier@math.tamu.edu }\end{array}$ & Tristan Rivière & $\begin{array}{l}\text { ETH, Switzerland } \\
\text { riviere@math.ethz.ch }\end{array}$ \\
\hline Igor Rodnianski & $\begin{array}{l}\text { Princeton University, USA } \\
\text { irod@math.princeton.edu }\end{array}$ & Wilhelm Schlag & $\begin{array}{l}\text { University of Chicago, USA } \\
\text { schlag@math.uchicago.edu }\end{array}$ \\
\hline Sylvia Serfaty & $\begin{array}{l}\text { New York University, USA } \\
\text { serfaty@ cims.nyu.edu }\end{array}$ & Yum-Tong Siu & $\begin{array}{l}\text { Harvard University, USA } \\
\text { siu@math.harvard.edu }\end{array}$ \\
\hline Terence Tao & $\begin{array}{l}\text { University of California, Los Angeles, USA } \\
\text { tao@math.ucla.edu }\end{array}$ & Michael E. Taylor & $\begin{array}{l}\text { Univ. of North Carolina, Chapel Hill, USA } \\
\text { met@math.unc.edu }\end{array}$ \\
\hline Gunther Uhlmann & $\begin{array}{l}\text { University of Washington, USA } \\
\text { gunther@math.washington.edu }\end{array}$ & András Vasy & $\begin{array}{l}\text { Stanford University, USA } \\
\text { andras@math.stanford.edu }\end{array}$ \\
\hline an Virgil Voiculescu & $\begin{array}{l}\text { University of California, Berkeley, USA } \\
\text { dvv@ math.berkeley.edu }\end{array}$ & Steven Zelditch & $\begin{array}{l}\text { Johns Hopkins University, USA } \\
\text { szelditch@math.jhu.edu }\end{array}$ \\
\hline
\end{tabular}

\section{PRODUCTION}

apde@mathscipub.org

Silvio Levy, Scientific Editor

Sheila Newbery, Senior Production Editor

See inside back cover or pjm.math.berkeley.edu/apde for submission instructions.

The subscription price for 2011 is US \$120/year for the electronic version, and \$180/year for print and electronic. Subscriptions, requests for back issues from the last three years and changes of subscribers address should be sent to Mathematical Sciences Publishers, Department of Mathematics, University of California, Berkeley, CA 94720-3840, USA.

Analysis \& PDE, at Mathematical Sciences Publishers, Department of Mathematics, University of California, Berkeley, CA 94720-3840 is published continuously online. Periodical rate postage paid at Berkeley, CA 94704, and additional mailing offices.

APDE peer review and production are managed by EditFLOW ${ }^{\mathrm{TM}}$ from Mathematical Sciences Publishers.

PUBLISHED BY

mathematical sciences publishers

http://www.mathscipub.org

A NON-PROFIT CORPORATION

Typeset in IATEX

Copyright (C2011 by Mathematical Sciences Publishers 


\section{ANALYSIS \& PDE}

\section{Volume $4 \quad$ No. $1 \quad 2011$}

Strichartz estimates on asymptotically hyperbolic manifolds

JEAN-MARC BOUCLET

Asymptotic behaviors of nonvariational elliptic systems

SZU-YU SOPHIE CHEN

Global regularity for the Navier-Stokes equations with some classes of large initial data

MARIUS PAICU and ZHIFEI ZHANG

Dynamics on Grassmannians and resolvents of cone operators

Juan B. Gil, Thomas Krainer and Gerardo A. Mendoza

Three-term commutator estimates and the regularity of $\frac{1}{2}$-harmonic maps into spheres 